\title{
Comparison of Marine Microalgae Culture Systems for Fuels Production and Carbon Sequestration.
}

\author{
GRANT NO. DE-FG02-02ER83515
}

SBIR PHASE II

Draft Final Report

May 30, 2006

SeaAg, Inc.

Dr. Joseph C. Weissman, Principal Investigator

and

Brooklyn College

Dr. Juergen Polle, Co-Principal Investigator

Contact : Joseph C. Weissman

SeaAg, Inc. 


\section{EXECUTIVE SUMMARY}

The dual problems of global fossil fuels supplies and global warming focus attention on the need to develop technologies that can provide large amounts of renewable fuels without contributing to global warming. The capture of power plant flue gas $\mathrm{CO} 2$ using microalgae cultures is one potential technology that could meet this objective. The central R\&D issues are the design and operation of low-cost algal mass culture systems and the development of algal strains and cultivation techniques that can achieve very high biomass productivities.

The major objective of this project was to develop mass culture techniques that could result in greatly increased biomass productivities, well above the about 50 metric tons per hectare per year (mt/ha/y) currently achievable. In this project, two marine microalgae species, the diatom Cyclotella sp.. and the green alga Tetraselmis sp., were cultivated on seawater in both open ponds and closed photo bioreactors, under a variety of different cultivation conditions. Simultaneous operation of the closed photo bioreactors and open ponds demonstrated similar productivities, under the same operating conditions. Thus the very expensive closed systems do not provide any major or inherent advantages in microalgae production over open ponds.

Mutants of Cyclotella sp. were developed that exhibited reduced pigment content, which theoretically would result in greatly increased productivities when grown under full sunlight. However, in open ponds, these mutant strains exhibited similar productivities as the parental strains. The mutant strains all grew relatively slowly, suggesting that additional mutations masked whatever inherent potential for increased productivities may have resulted from the reduced pigment content. Research is still required to develop improved low pigment strains.

When open pond cultures were exposed to intermittent sunlight, by partially covering the ponds with slats, solar conversion efficiencies increased dramatically, by over $50 \%$.

Although such techniques are not directly applicable to practical processes, the experiments demonstrated the inherent potential of algal mass cultures to achieve very high productivities.

Nitrogen limited pond cultures demonstrated that it is possible to produce biomass with a potentially high content of carbohydrates or oils (although these were not directly measured in these experiments), without reducing achievable productivities. This suggested that microalgae biomass suitable for conversion to biofuels (ethanol or biodiesel) could be produced without compromising productivity. Experiments combining both light modulation and nitrogen limitation indicated possibly synergistic effects.

The goal of developing practical and economic processes for the sustainable production of renewable fuels with microalgae pond cultures using power plant flue gases as sources of CO2 was advanced by these studies, but requires more work. Most important is the research, development and demonstration in outdoor pond cultures of algal strains with low pigment content. Such strains are the most likely approach to achieve, in combination with the other mass culture techniques investigated in this study, the very high productivities, above 100 $\mathrm{mt} / \mathrm{ha} / \mathrm{y}$ ( $45 \mathrm{t} / \mathrm{acre} / \mathrm{y}$ ), that are the goal in this field. The projected economics for such a process suggests that, as for higher plant biofuel production, microalgae biofuels production should be developed as a multiproduct process providing additional higher value co-products. 


\section{TABLE OF CONTENTS}

Executive Summary

Table of Contents

1. INTRODUCTION AND OBJECTIVES

2. MATERIAL AND METHODS

3. EXPERIMENTAL RESULTS

3. 1. Biomass Productivity: Error Analysis and Reproducibility of Results 3.1.1. Error Analysis

3.1.2. Reproducibility of Results

3.2. Media Optimization

3.2.1. Silicon Nutrition

3.2.2. pH Experiment

3.3. Vertical Flat Plate Photobioreactors vs. Open Raceway Ponds

3.4. Experiments with Photosynthetic Mutants

3.5. Intermittent Illumination of RW Ponds

3.6. Nitrogen Limited Productivity of Outdoor Raceway Ponds

3.7. Experiments with Nitrogen Limited and Intermittently Covered Ponds

4. CONCLUSIONS AND TECHNOECONOMIC ANALYSIS

5. REFERENCES

APPENDIX - REPORT BY PROF. J. POLLE, BROOKLYN COLLEGE 


\section{INTRODUCTION AND OBJECTIVES}

The goal of this project is to develop technology to increase biomass productivity of outdoor, micro algal cultures well beyond the approximately 50 to $60 \mathrm{mt} / \mathrm{ha} / \mathrm{y}$ currently achieved.

This is a central goal of microalgal mass cultures, in particular for production of biofuels and greenhouse gas abatement, where productivity is a major and necessary determinant of success (Weissman, 1978; Weissman and Goebel, 1987; Benemann and Oswald, 1996, Sheehan J et al, 1998).

The relationship between photosynthetic solar conversion efficiency, a direct measure of productivity, and the intensity of the light incident on the cultures is the basic issue that must be addressed to achieve this goal. Algal cells in mass cultures normally are physiologically adapted to low light intensity, in that they have large light absorbing pigment systems, socalled "light harvesting” or "antenna" pigments. The pigments are mainly chlorophyll (in green algae), fucoxanthin (in diatoms) or phycobiliproteins (in cyanobacteria), and are used by the photosynthetic system to capture (harvest) photons. The light harvesting pigments funnel the absorbed light energy to reaction centers where it is used to produce a primary charge separation. The strong oxidizers formed in the first of these systems (Photosystem II, PSII) take electrons from water, leading to the $\mathrm{O} 2$ evolution characteristic of green plants and microalgae. The reducing power generated by PSII is then used to produce both ATP (via a chain of membrane-bound electron transport enzymes) and, with a second photosystem (Photosystem I, PSI), reduced ferredoxin, which generates the reduced pyridine nucleotides (e.g. NADPH) used along with ATP in the dark reactions that convert CO2 to sugars, and also allow assimilation of nutrient and production of more cell mass, and cell reproduction.

At low light intensities, the conversion efficiency of photon energy into biomass energy (measured as the heat of combustion of the biomass) exceeds 20\% of visible light. This is equivalent to about $10 \%$ of sunlight energy converted to biomass. The fundamental problem in photosynthesis is that at light intensities of only about $10 \%$ of full sunlight intensity, the light harvesting pigments in the algal cells absorb as much light as can be processed by the reaction centers and the electron transport enzymes between the two photosystems.

However, light absorption does not stop when the electron transport system is saturated. In a dense algal mass culture, those microalgal cells near the culture surface are exposed to full sunlight, resulting in excessive light absorption, with up to $99 \%$ of the captured photons not able to be used, and leading to a rapid dissipation as heat (and some fluorescence) of the excess photon energy absorbed. This is the light saturation effect (Kok, 1953; Myers,1957).

Even when algal cells are grown as dilute cultures (so they only minimally shade each other) photosynthesis reactions, most easily measured as $\mathrm{CO} 2$ fixation and $\mathrm{O}_{2}$ evolution, saturate at a light intensity of only about $10 \%$ of full sunlight, Thus exposure to higher light results in waste of photon energy. In any event, dilute cultures are not practical as they cannot capture all of the sunlight, a requirement for any solar converter.

When algae are grown in photo bioreactors (e.g. open ponds or enclosed bioreactor) for production of biomass and other products, the algal cell numbers increase until a dense 
suspension is achieved which absorbs essentially all of the incident light, a requirement for high productivity (and thus high solar conversion efficiency). In such cultures each cell's photosynthetic apparatus is subject to the light saturation phenomenon described above. The incident solar radiation impinging on the top layers of algal cells, exposed to the highest solar flux, is used at very low efficiency, as most, typically $90 \%$, of the absorbed photons are dissipated as heat. Cells deeper in the culture, being shaded by the cells above them, do not receive as many photons, and, thus, use the light they receive relatively more efficiently. Cells near the bottom of the culture, receive very few photons, which they use very efficiently, although their rate of photosynthesis can be often below that of respiration.

Overall, productivity, that is the depth integrated solar conversion efficiency, is disappointing. A large part, from about two-thirds to as much as four-fifth of the incident light energy is wasted shortly after absorption and prior to any further processing by the photosynthetic apparatus (Bush, V 1953). This greatly diminishes solar conversion efficiency from its theoretical maximum of about $12 \%$ (Weissman, 1978) to, at best, typically $2 \%$, and at most close to $3 \%$, of total solar energy into biomass. How to overcome this fundamental limitation on productivity is a problem addressed by this project.

Several methods are available to overcome this limitation. If the algal suspensions are mixed intensely enough, cells will move in and out of the highly illuminated zones of the cultures at such a rate that the light energy absorbed by the PSII and PSI reaction centers can be processed in the dark zones (that is, the reaction centers primary electron acceptors can be oxidized via electron transport from PSII to PSI and from PSI to reduce NADP, before more photons are absorbed). Unfortunately, the time constants for the electron transport reactions, most critically between PSII and PSI, and thus the required speed for the algal cells to move in and out of the light, are relatively short, on the order of only a few milliseconds. Kok (1953) demonstrated that for cultures exposed to high light intensities for short periods (one to two milliseconds), followed by a dark phase about five times as long, the efficiency of light utilization approached that of the cells grown under continuous low light.

Unfortunately, achieving this "flashing light” effect with mass cultures requires such intense mixing of the cultures that it is impractical for anything but laboratory demonstrations $(\mathrm{Hu}$ and Richmond, 1996, Barbosa et al 2003).

A second solution is to diffuse light from the surface to the interior of the culture, by means of prisms (Kok, 1953) or, as proposed for several decades, by using concentrating mirrors coupled to optical fibers, which introduce a diluted light into an enclosed photo bioreactor. The latter approach was the basis for a major Japanese program and also a recent project in the U.S. (Bayless, 2005). However, the unavoidable fact is that such systems have extraordinarily high costs in the concentrating mirrors and accessory equipment, so as to make this approach impractical by many orders of magnitude.

Another, somewhat more practical, solution is to orient closed photo bioreactors vertically, so as to lower the light intensity incident on their surfaces. This, however, requires multiple reactors per unit area of ground surface to allow interception of all the sunlight (similar to the leaf index for higher plants). Further, this approach is limited by many practical issues in photo bioreactor design, and economics would not justify multiple reactors per unit area in any event. The relatively small individual size of such closed photo bioreactors does not 
allow for economies of scale, which can be achieved with simpler open ponds (Weissman al., 1987, Weissman and Tillett, 1992). Closed photo bioreactors of any type are still much too expensive for applications in greenhouse gas abatement and biofuels production. Vertical or inclined closed photo bioreactors (tubular of flat plate) are often reported to have much greater productivity than open ponds, with this difference generally attributed to an inherent advantage of closed reactors, above and beyond their physical orientation (e.g. light interception per unit area). For example, the ability of such closed systems to better control conditions, such as the supply of $\mathrm{CO} 2$ and $\mathrm{pH}$, temperature, improved gas exchange, avoidance of contaminations, higher cell concentrations, etc., have all been cited as reasons for such higher productivity, along with better light distribution and modulation within the closed photo bioreactors. However, actual side-by-side comparisons between open ponds and closed photo bioreactors have been lacking. This project addressed this issue by performing experiments to determine if improvements in photosynthetic efficiency achieved with vertical photo bioreactors vs. ponds could be accounted for only due to the difference in light capture due based on orientation (vertical vs. horizontal).

Another, more general, and powerful, solution to the problem of light saturation is to find or create algal strains with reduced amounts of light harvesting pigments. Such cells would absorb many fewer photons, bringing the rate of photon absorption closer to the rate at which the absorbed energy can be used, and allow other cells in the water column to beneficially use the remaining photons. Theoretical calculations demonstrate that integrated over the entire culture, such algal strains, with reduced light harvesting pigments, should exhibit a $300 \%$ or greater increase in productivities in algal mass cultures (Weissman, 1978).

Developing such strains of algae was a major goal of this project.

Of course, to achieve maximal productivity, the algal suspensions must be held at optimal temperature with all nutrients supplied in sufficient amounts. Temperature will limit the locations where such systems can be established, while optimal nutrient supply is not a major limitation in open pond systems. A greater challenge is that the algal strains must be able to be maintained in the mass culture, against invasion by normal ("wild type") cells. It must be recognized that such normal cells have a strong competitive advantage over strains with smaller antenna pigment complexes, as they grow better at low light intensities, which is typically the condition most of the time for cells growing in dense cultures. This problem could be overcome by re-starting the cultures with fresh inoculum whenever contamination appears, however contamination is still a challenge in the application of such strains.

In addition to the light saturation effect, algal photosynthesis is also limited by light inhibition: at light intensities much higher than saturating, light becomes inhibitory, due to the damage from excess photons absorbed that cannot be used in photosynthesis and are dissipated through various side reactions. This inhibition can be a short term, observed as a reversible lowering of photosynthesis (measured as $\mathrm{CO} 2$ fixation or $\mathrm{O} 2$ production), or, can be a longer-term irreversible destruction of the photosynthetic machinery, eventually leading to cell death. The algal cells have regulatory processes that shut down photosynthesis to protect the reaction centers from the intense bombardment of photons. One interesting issue is whether the reduced antenna strains would also exhibit reduced light inhibition. 
In this project, paddlewheel mixed, raceway type open (RW) ponds and vertical flat-plate (VFP) closed photo bioreactors (made with a plastic bag hung between constraining fences) were evaluated to determine the specific factors which accounted for biomass productivity e.g. simple geometry (leading to light dilution in vertical photo bioreactors) or, possibly, other properties of such photo bioreactors. In brief, it was demonstrated that the fundamental features which limit photosynthetic productivity are independent of the type of photo bioreactor used. Compared to RW open ponds, VFP photo bioreactors achieved higher solar conversion efficiencies (but lower productivities) per total bioreactor surface area in the summer, when incident light intensity is lower on the surface of these reactors. Of course, such an orientation wastes a large fraction of the incident photons when the sun is high in the sky. The opposite was true in the winter, when vertically oriented reactors had lower efficiencies (but higher productivities) than open ponds. The major advantage of the closed systems was that temperature could be controlled more optimally using water sprays to cool the reactors in summer, and heat them in winter. The disadvantage was the limitation on scale-up of closed systems (Weissman et al., 1987) and the constant effort required to keep the walls free of growth which blocked light.

Other means were developed to increase photosynthetic efficiency. By partially covering an open pond system intermittently, higher photon conversion efficiency was achieved (per incident photons received by the uncovered area of the ponds). Of course, since much of the pond area was covered, this is not a practical approach. However, these experiments demonstrated that it is in principle possible to significantly increase photosynthesis by modulating the light regime received by the cells. As the time constants for cells moving in and out of the light in this case were a hundred times longer than applicable to the light saturation effect, this suggested a different mechanism, specifically photoinhibition, as a likely explanation for these results. A detailed study of the effect of intermittent light on photosynthetic efficiency was initiated.

The main objective of this project was to find ways to increase not just photon conversion efficiency but also overall productivity. When dense algal cultures with highly pigmented cells are greatly diluted, individual cells are then continuously exposed to very high light intensities which inhibits the synthesis of light harvesting pigments and leads to cells having reduced antenna sizes. This is the phenomenon of photoadaptation. This condition persists until growth re-establishes high densities. During this transition, a period of typically a day or less, during which the cells are less pigmented than normal, it may be possible to demonstrate an increase in the light intensity at which photosynthesis saturates. However, as stated above, the most direct, and permanent, way to achieve to this objective, would be to alter the photosynthetic machinery of the algae by reducing the amounts of light-harvesting pigments. This was attempted by means of mutagenesis and screening in the laboratory for low pigment cells of microalgae strains able to grow in the field. Thus the first step was to screen strains for the ability to grow in stable cultures in either the RW open ponds or the VFP photo bioreactors. From these strains many mutants were produced and screened in the laboratory, and the most promising ones were studied in the outdoors field reactors. The wild-type strains were further characterized in terms of maximum growth rate in the field reactors and optimal nutrient levels needed to maximize biomass production. 
In outdoor experimentation, complete control of the environmental conditions is impossible. Both temperature and insolation vary continuously. The values of these variables must be taken into consideration when interpreting each experiment. Given the intrinsic difficulty in running and interpreting experimental data, it is imperative that the results be reliable and reproducible. To assure this, both an in-depth error analysis and several reproducibility experiments were performed. These experiments are discussed first, after the Methods. 


\section{MATERIAL AND METHODS}

Microalgae Culture. Unless otherwise stated all experiments reported used a culture of Cyclotella sp. (strain CYCLO J, and subcultures, as noted) was used that has been cultured in outdoor ponds at SeaAg, Inc. for several years. The other organism used, where stated, was Tetraselmis sp., strain T- BCA-1, a green algal strain obtained from the co-PI, J.Polle.

Bioreactors. Figure 1 shows the two types of algae culture devices used in outdoor cultivation: RW (paddlewheel mixed, raceway) ponds and VFP (vertical flat plate) photo bioreactors. The RW are $2.8 \mathrm{~m} 2$ in size, and have been used extensively in prior projects. The VFP reactors, one provided by Prof. Mario Tredici of the University of Florence and the other replicated at SeaAg, Inc., are $0.63 \mathrm{~m} 2$ in size at a depth of $77 \mathrm{~cm}$.

Media. Saline, groundwater was used for all cultivation at the field site located at SeaAg, Inc., Fort Pierce, Florida. Salinity was relatively constant at $27-30 \mathrm{mg} / \mathrm{L}$. Standard, commercial concentrates of Guillard's $f / 2$ medium was used for stock cultures. To sustain the biomass productivities attained in outdoor reactors, the saline groundwater was supplemented with urea, iron sulfate (septahydrate), and phosphoric acid. For diatoms this media were additionally supplemented with sodium metasilicate pentahydrate (neutralized with sulfuric acid), as needed.

Pond operations. The objective was to evaluate the biomass productivity of stable cultures of microalgae to allow extrapolation of results large-scale cultivation. For practical applications some type of continuous cultivation is required; at this experimental scale, semi continuous once-per day dilution, is easy to perform and can be used to approximate large-scale cultures. With this method, each morning a prescribed proportion of the pond volume (determined as depth) was removed and replaced with fresh medium. The daily operations were carried out as follows: a) take temperature, $\mathrm{pH}$, and depth measurements; b) add fresh water to make up for any evaporation (e.g. bring the pond depth back to the prescribed level with fresh water); c) take a one liter sample of the culture; d) remove a predetermined fraction of the culture to reduce the pond level to the new, desired depth; $\mathrm{f}$ ) refill with saline ground water to the standard depth; g) add nutrients as required These methods are applicable regardless of reactor type.

Biomass Productivity. Biomass productivity for the horizontal RW pond reactors is calculated as grams of biomass per square meter of horizontal surface per day. For a given day this is calculated as the standing biomass (grams per square meter) of the culture after dilution, subtracted from the standing biomass the next morning before dilution. The standing biomass is calculated from the biomass density (grams ash free dry mass, AFDM, per liter) times the liters per square meter of pond surface. For the vertical reactor there is a small amount of light impinging on the "back" side of the reactor. This is less than $5 \%$ of that on the south facing side, so it was ignored in the calculations that used the area of only one side of the reactor. Biomass was converted to energy content using a conversion of 5.5 kcal per gram of AFDM. 
Light Measurements and Photosynthetic Efficiency. Li-Cor SA 200 pyranometers were used to measure total solar insolation. One was mounted on a level horizontal surface, another was mounted facing south on a vertical surface. The former would thus measure the insolation on a horizontal reactor, e.g., a RW pond. The latter measures the insolation on a south facing vertical reactor, e.g., the VFP reactor. In the summer, the south facing vertical sensor was moved to face east, and an additional vertical sensor was faced west. These were used to measure light incident on both side of vertical reactors aligned north-south so that their sides faced east and west. The sensors were linked to a data logger which logged daily insolation as watt-hours per square meter per day, i.e., W.hr.m-2.d-1. [NOTE: on many of the graphs and tables this is erroneously written as W.hr-1.m-2.d-1]. This was converted to kcal per square meter per day $(1$ watt-hour $=0.86 \mathrm{Kcal})$, which was then used to calculate photosynthetic efficiency (PE) from the above calculated biomass productivity, using 5.5 kcal.g-1 for the heat of combustion of the algal biomass. Unless otherwise noted, all photosynthetic efficiencies are stated in terms of PAR, the photosynthetically available radiation, which corresponds to the visible part of sunlight and which was assumed to be $44 \%$ of the total solar radiation. Total solar conversion efficiencies are thus 2.2-fold lower.

Microscopic examination. Cultures were examined under a microscope three times per week to determine culture characteristics including presence of grazers, clumps, and species of algae present.

Ash free dry mass determination. Samples were taken for AFDM measurement every day. Enough volume of sample was filtered over a pre-ashed, pre-weighed Whatman GF/C filter to accumulate about $10 \mathrm{mg}$ AFDM. Weights were measured using an analytical balance which measures to $0.01 \mathrm{mg}$. With this balance and this amount of biomass, replication was generally to within 3\%. Filtered samples were rinsed with a $20 \mathrm{~g} / \mathrm{L}$ solution of ammonium formate prior to drying to remove salts. Samples were dried at $103 \mathrm{C}$ over night, and ashed for 15 minutes at $550 \mathrm{C}$. Results were expressed as mg/L.

The formulas used to calculate derived quantities, for the specific depths shown, are given in Table 1. The raceway pond reactors used in the work are shown in Figure 1. 


\section{Table 1. \\ Worksheet Formulas \\ Semi-continuous Cultures.}

\section{Flat Plate}

Volune: 49 Liter

Surface Area/side : $0.63 \mathrm{~m}-2$

Running vertical depth: $77 \mathrm{~cm}$

Standing Biomass Per Pond (BD) $(\mathrm{g})=($ AFDM mg.L-1 * (Beginning Depth $\mathrm{cm} / 77 \mathrm{~cm}) * 49 \mathrm{~L}) / 1000$

Standing Biomass Per Pond (AD) $(\mathrm{g})=($ AFDM mg.L-1 * $($ Lowered Depth $\mathrm{cm} / 77 \mathrm{~cm})$ * $49 \mathrm{~L}) / 1000$

Daily Biomass Production (1 side surface area) (g.m-2.d-1) $=$ (Standing Biomass per Pond (BD) Standing Biomass per Pond (AD)) / 0.63

Daily Biomass Production ( 2 side surface area) (g.m-2.d-1) $=$ (Standing Biomass per Pond (BD) Standing Biomass per Pond (AD)) / 1.26

Depth Adjusted AFDM (mg.L-1) $=($ Beginning Depth $\mathrm{cm} / 77 \mathrm{~cm}) *$ AFDM mg.L-1

Energy in Light (kcal.m-2.d-1) $=\left((\text { AM Insulation + PM Insulation w-hr.m-2.d-1) * } 3600 \mathrm{~s} / 4.19 \mathrm{~J} / 1000)^{*} 0.45\right.$

Energy in Biomass (kcal.m-2.d-1) $=($ Daily Biomass Production $) *(5.5 \mathrm{kcal}$ per gram of dry algae biomass $)$

Photosynthetic Efficiency - PE (\%) = Energy in Biomass $) /($ Energy in Light $) * 100$

\section{Raceway Ponds}

Volume: 420 Liter at $15 \mathrm{~cm}$ depth.

Surface Area: $2.8 \mathrm{~m}-2$

Running Vertical Depth: $15 \mathrm{~cm}$

$\underline{\text { Standing Biomass (BD) (g.m-2) }}=($ Beginning Depth $\mathrm{cm}$ * 10 liters * AFDM mg.L-1) $/ 1000$

Standing Biomass (AD) (g.m-2) $=($ Lowered Depth $\mathrm{cm}$ * 10 liters * AFDM mg.L-1) / 1000

Daily Biomass Production (g.m-2.d-1) $=($ Standing Biomass (BD) - Standing Biomass (AD))

Depth Adjusted AFDM (mg.L-1) $=($ Beginning Depth $\mathrm{cm} / 18 \mathrm{~cm}){ }^{*}$ AFDM mg.L-1

Energy in Light (kcal.m-2.d-1) $=\left((\text { AM Insulation + PM Insulation w-hr.m-2.d-1) * } 3600 \mathrm{~s} / 4.19 \mathrm{~J} / 1000)^{*} 0.45\right.$

Energy in Biomass (kcal.m-2.d-1) $=($ Daily Biomass Production $) *(5.5 \mathrm{kcal}$ per gram of dry algae biomass $)$

Photosynthetic Efficiency - PE (\%) = Energy in Biomass) / (Energy in Light) * 100

BD - Before Dilution $\quad$ AD - After Dilution 
Figure 1.

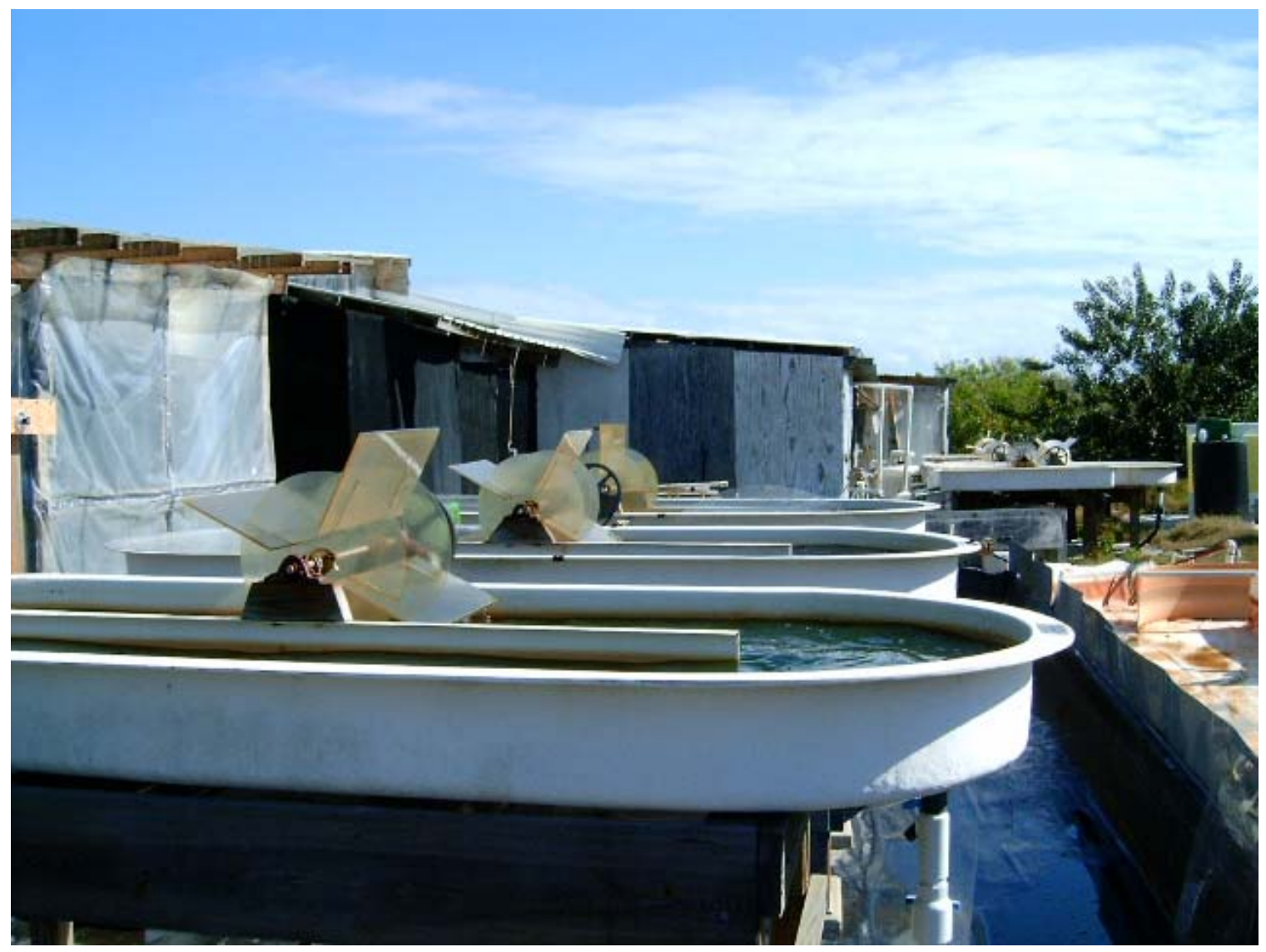


Figure 1. continued.
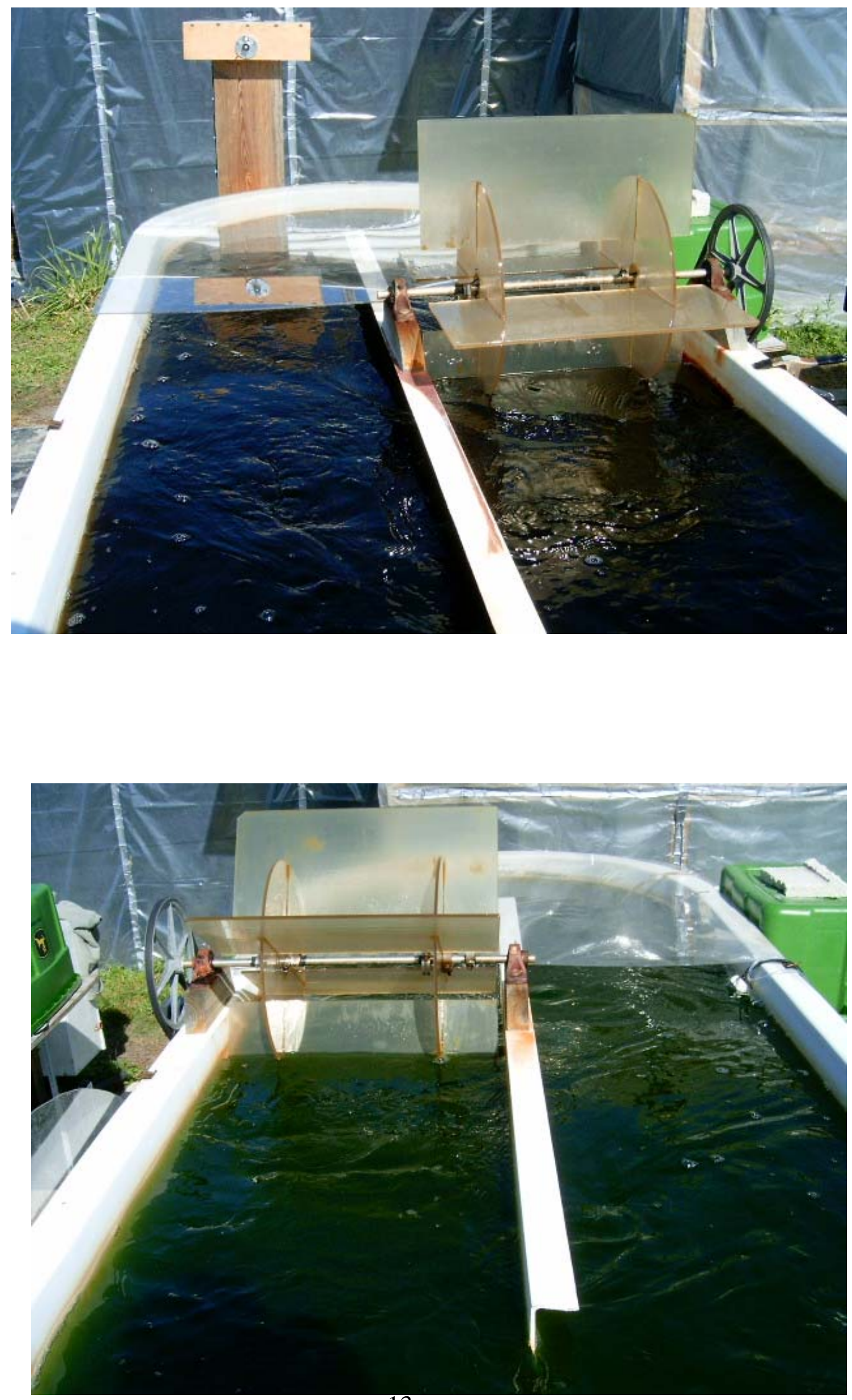
Figure 1 Continued. Vertical, Flat Plate Photobioreactors

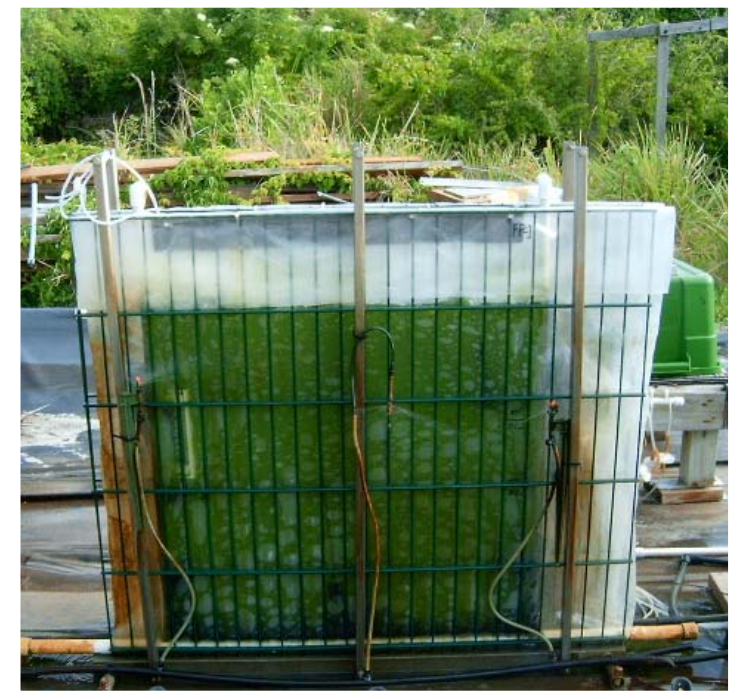

FP-1

$4 / 27 / 2004$

Tetraselmis sp (T-BCA-1), Run 12

$\mathrm{AFDM}=315$

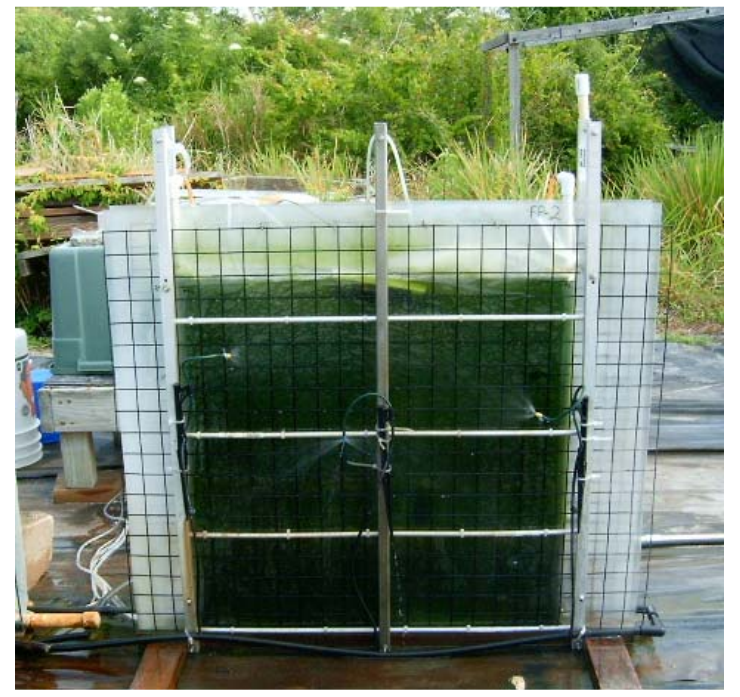

FP-2

$4 / 27 / 2004$

Tetraselmis sp (T-BCA-1), Run 13 $\operatorname{AFDM}=450$

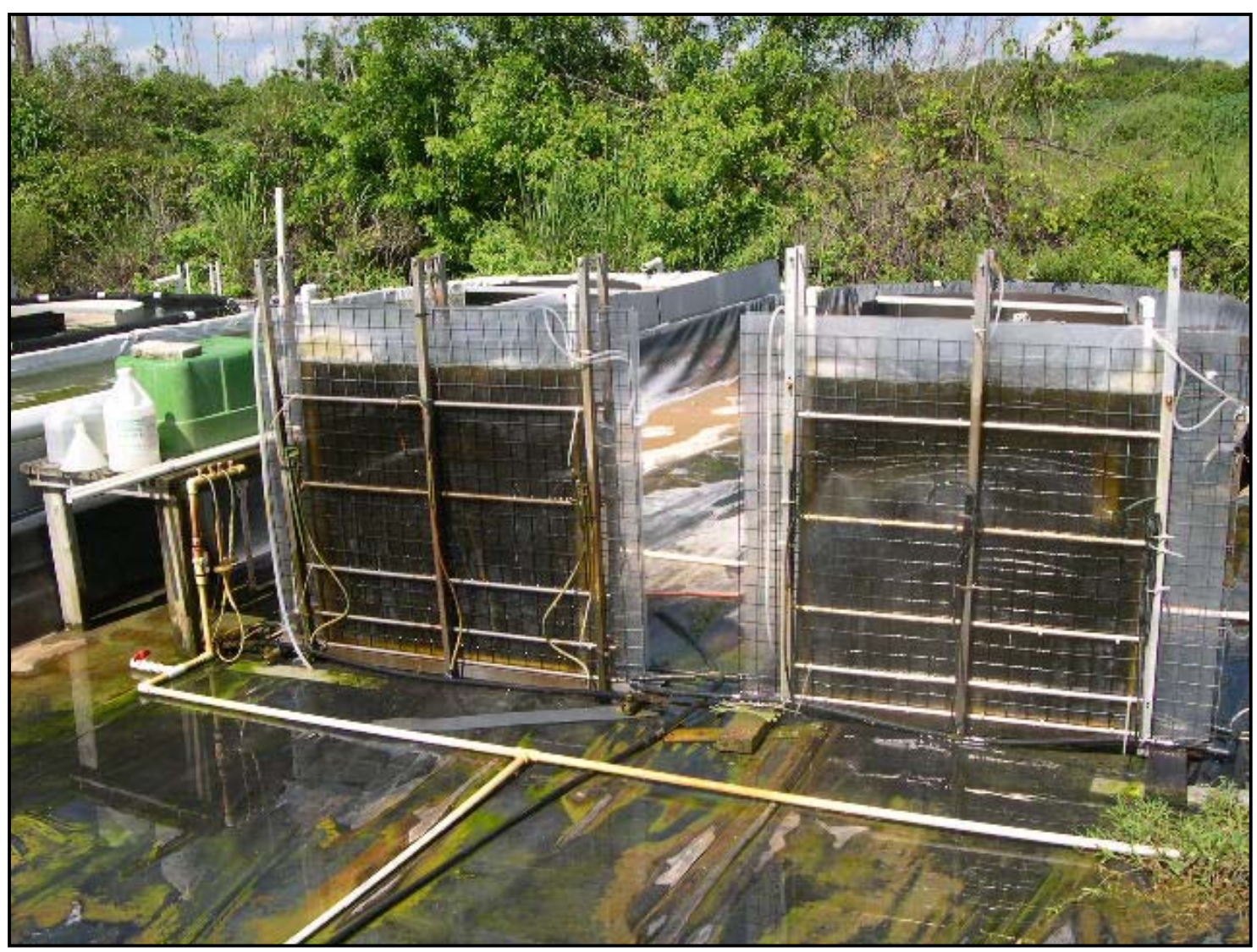

Vertical Flat Plate Bioreactors

Cyclotellis sp. 


\section{EXPERIMENTAL RESULTS}

\section{1. Biomass Productivity: Error Analysis and Reproducibility of Results}

\subsubsection{Error Analysis}

At first glance, it might seem that the uncertainty associated with the measurement of biomass productivity should be the standard deviation of a number of such measurements. Clearly, though, the uncontrollable inputs of insolation and temperature cause variability that is not associated with the uncertainties in the measurements themselves.

Several variables were measured for the calculation of the biomass productivity. There are uncertainties in each of these measurements, as shown in Table 2, which are propagated in the calculation. The error propagation is calculated using the sum of quadratures expression which is the square root of the sum of the squares of the uncertainty due to each variable. The uncertainty in a function due to a particular variable is the partial derivative of the function with respect to that variable multiplied by the uncertainty in the variable. To begin this analysis, the formula for biomass productivity is first presented, and then the uncertainty in each of the measured variables is discussed.

The biomass productivity on a given day, day n, was calculated as the standing biomass (biomass per unit area, gm-2) before dilution on the following morning, day $n+1$, minus the standing biomass after dilution on day $\mathrm{n}$. The ponds were diluted each day in the morning, after sampling. The sample on day $n+1$ provided the biomass density measurement for the calculation of standing biomass at the end of the previous photosynthetic day (day n) and for the calculation of standing biomass for the beginning of day $n+1$. (Of course, respiration during the night will reduce the actual biomass productivity, but this is incorporated into the analysis implicitly). The depth before dilution and the depth after removing the pond volume for that day were used in these calculations. Standing biomass was calculated as the ash free dry mass, in mg.l-1, times the volume at the appropriate depth divided by the pond area. This can be rewritten as dry mass times a factor, F, times the pond depth, as measured in $\mathrm{cm}$. The factor has units of 1.cm-1.m-2: liters of pond volume per $\mathrm{cm}$ of pond depth per $\mathrm{m} 2$ of pond area. If the pond were exactly flat bottomed and had exactly straight side walls, this factor would be equal to the number 10 liters per $\mathrm{cm}$ per $\mathrm{m} 2$. Since this is not the case, we measured the volume per pond as a function of depth. The result is $28.1 \mathrm{l} . \mathrm{cm}-1 \pm 0.21$ (0.7\%), as shown in Table 3.

The uncertainty in the measurement of depth was determined by repeatability. Depth was measured many times with a $30 \mathrm{~cm}$ plastic ruler, graded in millimeters. The value for the depth could be read to within plus or minus one millimeter. The paddlewheel was turned off when depth was measured otherwise the uncertainty would have been more than twice as high. Thus the relative uncertainty in the measurement of depth depends on the value of depth. Typically it was about $1 \%$ as explained in Table 2.

The area of the ponds had been measured previously very accurately using a paper trace of the surface. The result was $2.8 \pm 0.03 \mathrm{~m} 2$. 
The relative error in the biomass concentration, measured as the dry weight (actually mass), is higher for very low than for greater dry weights. Thus for dry weights between 0-4.9 mg.l1 , the relative error is $3.4 \%$, for 5.0 to $50 \mathrm{mg} . \mathrm{l}-1$ it is $1.5 \%$, and for greater than $100 \mathrm{mg} . \mathrm{l}-1$ is levels off to about $1 \%$. This was determined from tabulating the results from many assays.

Finally, the uncertainty in the time interval between sampling has to be considered. The samples were taken 24 hours apart plus or minus 30 minutes. Based on a photosynthetic day of 12 hours, the relative error is $4 \%$. However, the photosynthetic production early in the morning is not nearly as much as later in the day, so the error in production is not linear with time. We arbitrarily discounted the uncertainty in time interval by half, resulting in a relative error of $2 \%$.

To summarize, the daily productivity is the difference between two values of standing biomass. Each determination of standing biomass, in g.m-2, is the dry weight times a volume to area factor times a depth. The starting standing biomass, on day $\mathrm{n}$, is the dry weight on that morning times the depth to which the pond is lowered times the average volume per cm per $\mathrm{m} 2$ at that depth. The ending standing biomass on day $n+1$ is the dry weight on that day times the depth before lowering the level for dilution times the average volume per $\mathrm{cm}$ per $\mathrm{m} 2$ at that depth. We assume these volumes to be the same to a specified degree of precision. The daily productivity is the difference divided by the time interval, in days. In propagating errors of products in a formula, the relative errors of the variables add in quadrature. For sums or differences (like the difference in standing biomasses), it is the absolute errors that add in quadrature. Both types of operations are involved in the calculation of biomass productivity.

Tables 4 and 5 give all of the error propagation formulas using the estimates of error just reviewed. Averages for the uncertainties in starting and ending standing biomass are used in the sample calculations for estimating the uncertainty in biomass production in these tables. To be more proper, for each measurement of biomass productivity, there are two calculations of standing biomass involved, each with an uncertainty associated with it. Tables $6(\mathrm{BD}=$ before dilution), 7 ( $\mathrm{AD}=$ after dilution), and 8 (Biomass Productivity) give the standing biomasses before and after dilution and biomass production from a typical run, with all of these uncertainties propagated to yield a final uncertainty for each value of production.

Productivities within the same range do not necessarily have the same error associated with them, unlike the case of the dry weight measurements. Thus in Table 9, the first and second productivities seem to yield inconsistent errors. The reason for this is that the uncertainty in biomass productivity depends on the difference between two numbers (starting and ending standing biomass) which may be large or small. The lowest productivity range (0.0 - 0.99) corresponds to days in which there was almost no production, but the standing biomasses were large. The error in the difference between two large numbers that are similar in value is relatively large. The next range of productivity $(1.0-1.99)$ corresponds to the first day of growth of cultures that were started very dilute (4 mg.l-1 dry weight). The standing biomasses involved were small numbers, but their difference was about of the same magnitude. Therefore the error in each standing biomass was small (the relative error in the 
measurement of all standing biomass values were about the same since it is derived from a product of the same variables). Thus the error in the difference between these low values of standing biomass was small, resulting in a small absolute error in productivity. These two competing influences, errors in large numbers with small differences and errors in small numbers with relatively large differences, serve to make all of the uncertainties in low to moderate productivities similar. As the productivities increase, the error settles down to a near constant value reflecting an error in the difference of large numbers where the difference is relatively large.

From Table 9, it can be seen that typically the uncertainty in biomass productivity is less than 1 g.m-2.d-1. 


\section{Table 2. Uncertainties in Variables Used to Calculate Biomass Productivity}

Uncertainty in Depth Measurement

All depth measurements are taken with same style ruler with $0.1 \mathrm{~cm}$ increments.

This is an approx $0.7 \%$ uncert at a depth of $15.5 \mathrm{~cm}$ and $1.2 \%$ uncet at a $7.5 \mathrm{~cm}$ lowered depth (50\% dilution)

An average $\%$ uncertanty of depth measurement will be considered to be $1 \%$

Uncertainty in Area Measurement

Pond surface area was measured to the nearest $0.1 \mathrm{~cm}$ at the approximate running depth of $15.5 \mathrm{~cm}$ (measured at drain end). Surface area of pond was measured to be $2.8 \mathrm{~m}-2$ with a $1 \%$ error

Uncertainty in Time of Dilutions

Ponds are sampled within a 5 min window prior to dilution. This delay in sampling time is so small that no error compensation will be made for delay in time interval between pond samplings.

All ponds are sampled between 8:00 and 9:00 (typically 9am). This approx. $1 \mathrm{hr}$ difference is adjusted downward since photosynthetic activity is low in the morning.

Uncertanty in time of dilutions will be considered to be $2 \%$

Uncertainty in Dry Weight Measurements

Error in dry weights were determined by analysing data from the month of July 2004 . Dry weights were arbitrarily put into catagories of $50 \mathrm{mg}$.L-1. (I.e. 1-49, 50-99, 99-149). Two samples from each pond were run daily, an average and stdev of that average were determined.

This stdev was then experess as a \% of that day's average AFDM. Then an average \% error was determined for each $50 \mathrm{mg}$.L- 1 range.

This \% error was highest for the lowest AFDM's but remained relatively steady for AFDM's in the range of values (100 to $300 \mathrm{mg}$.L-1) Used in average productivity calculations.( 1\%). Error rates for all AFDM calculations are thus assumed to be $1 \%$.

$\begin{array}{llllll}\text { mg.L-1 } & \delta & \% \delta & \text { mg.L-1 } & & \% \\ \text { Range } & \text { AFDM } & \text { AFDM } & \text { Range } & \delta \text { AFDM } & \text { AFDM } \\ 0-4.99 & 0.10 & 3.37 & 50-99 & \pm 1.13 & 1.51 \\ 5-9.99 & 0.11 & 1.51 & 100-149 & \pm 0.89 & 0.71 \\ 10-14.99 & 0.20 & 1.58 & 150-199 & \pm 1.68 & 0.98 \\ 15-19.99 & 0.23 & 1.31 & 200-249 & \pm 2.33 & 1.05 \\ 20-24.99 & 0.16 & 0.70 & 250-299 & \pm 2.36 & 0.84 \\ 25-29.99 & 0.18 & 0.66 & & & \mathbf{0 . 8 9 5}\end{array}$

Uncertainty in Insolation measurements

The Li-cor meter records continuously from 12:01 to $2359 \mathrm{hrs}$. Manufacturer reported \% error is $5 \%$ 
Table 3

\section{Estimation of Error in Pond Volume}

Volume of Ponds = This is the error in determining the L.cm-1 in a pond.

Ponds were measured by adding water into empty pond @ 20L increments and measuring water depth to the nearest $0.1 \mathrm{~cm}$ at the drain end of the pond. Depth measurements were recorded every 60L.

Pond D5

Volume Depth L.cm-1

\begin{tabular}{ccc}
$(\mathrm{L})$ & $(\mathrm{cm})$ \\
\hline 420 & 15 & 28.00 \\
360 & 12.7 & 28.35 \\
300 & 10.5 & 28.57 \\
240 & 8.5 & 28.24 \\
180 & 6.4 & 28.13 \\
\hline & Avg & 28.26 \\
& St Dev & \pm 0.22
\end{tabular}

Pond D2

\begin{tabular}{ccc}
$\begin{array}{c}\text { Volume } \\
(\mathrm{L})\end{array}$ & $\begin{array}{c}\text { Depth } \\
(\mathrm{cm})\end{array}$ & L.cm-1 \\
\hline 420 & 14.9 & 28.19 \\
360 & 12.8 & 28.13 \\
300 & 10.7 & 28.04 \\
240 & 8.6 & 27.91 \\
180 & 6.5 & 27.69 \\
\hline & Avg & 27.99 \\
\multicolumn{3}{c}{ St Dev \pm 0.20}
\end{tabular}

Average L. cm-1 = 28.1 L.cm-1 \pm 0.21

$\%$ vol error $=0.21 / 28.1=0.7 \%$ 


\section{Table 4}

\section{Estimation of Error in Standing Biomass}

Formulas for calculating Standing Biomass $\mathbf{x}$

Standing Biomass $\mathrm{BD}_{\mathrm{BD}}(\mathbf{g} \cdot \mathbf{m}-\mathbf{2})=$ Standing biomass before dilution $=\left(\mathrm{AFDM}_{\mathrm{AVG}} \mathrm{D}\right.$ mg.L-1 X F $\mathrm{F}_{\mathrm{V} / \mathrm{A}}$ L.cm-1.m-2 $\times$ Beg. Pond Depth $) / 1000$ $F_{V / A}=$ Volume/Area Factor, assumes there is $10 \mathrm{~L}$ water/m-2 surface area @ 1cm of depth. (10L.cm-1.m-2)

Standing Biomass ${ }_{A D}(\mathbf{g} \cdot \mathbf{m}-2)=$ Standing biomass after dilution $=\left(\right.$ AFDM $_{A V G ~}$ mg.L-1 $X F_{V / A}$ L.cm-1.m-2 $\times$ Lowered Pond Depth $\left.{ }_{D}\right) / 1000$ $\mathrm{F}_{\mathrm{V} / \mathrm{A}}=$ Volume/Area Factor, assumes there is $10 \mathrm{~L}$ water/ $\mathrm{m}-2$ surface area @ 1cm of depth. (10L.cm-1.m-2)

Formulas for calculating error in Standing Biomass ${ }_{x}$

$\delta$ "value" = Absolute uncertanty of "Value" or error of "Value"

R $\delta$ "Value" = Relative Error of "Value" or ( $\delta$ "value" / "value" )

$\mathbf{R} \delta$ Standing Biomass $\mathrm{X}=\mathrm{V}(\mathrm{R} \delta \mathrm{AFDM})^{2}+\left(\mathrm{R} \delta \mathrm{F}_{\mathrm{V} / \mathrm{A}}\right)^{2}+(\mathrm{R} \delta \text { Depth })^{2}$

$R \delta F_{V / A}=v(R \delta \text { "Volume" })^{2}+(R \delta \text { Area })^{2}$

$\mathrm{R} \delta$ "Volume" = ( $\delta$ "Volume" L.cm-1/ "Volume" L.cm-1)

"Volume" $=($ Depth $\mathrm{cm}$ X Avg L.cm-1) / (depth cm) or 28.1L.cm-1

$\mathrm{R} \delta$ Area $=(\delta$ Area $/$ Area $)$

$R \delta$ AFDM $=(\delta$ AFDM $/$ AFDM $)$

$\mathrm{R} \delta$ Depth $=(\delta$ Depth/ Depth $)$

$\delta$ Standing Biomass $\mathrm{x}=(\mathrm{R} \delta$ Standing Biomass $\mathrm{X}) \mathrm{X}($ Standing Biomass $\mathrm{X})$

Sample calculation of the $\mathrm{R} \delta$ Standing Biomass $\mathrm{x}$

$R \delta$ Standing Biomass BD $=v(R \delta \text { AFDM })^{2}+\left(R \delta F_{V / A}\right)^{2}+(R \delta \text { Depth })^{2}=v(0.01)^{2}+(0.014)^{2}+(0.01)^{2}=\mathbf{0 . 0 2}$

$R \delta F_{V / A}=v(R \delta \text { "Volume" })^{2}+(R \delta \text { Area })^{2}=v(0.01)^{2}+(0.01)^{2}=0.014$

$\mathbf{R} \delta$ Standing Biomass AD $=\mathrm{v}(\mathrm{R} \delta \mathrm{AFDM})^{2}+\left(\mathrm{R} \delta \mathrm{F}_{\mathrm{V} / \mathrm{A}}\right)^{2}+(\mathrm{R} \delta \text { Depth })^{2}=\mathrm{v}(0.01)^{2}+(0.014)^{2}+(0.01)^{2}=\mathbf{0 . 0 2}$

$R \delta F_{V / A}=v(R \delta \text { "Volume" })^{2}+(R \delta \text { Area })^{2}=v(0.01)^{2}+(0.01)^{2}=0.014$

Sample calculation of the $\delta$ Standing Biomass ${ }_{x}$

$\delta$ Standing Biomass $\mathrm{BD}_{\mathrm{BD}}(\mathbf{g} \cdot \mathbf{m}-\mathbf{2})=(\mathrm{R} \delta$ Standing Biomss $X) X($ Avg Standing Biomass $)=(0.014) X(34.43)=\mathbf{0 . 6 9}$ g.m-2

$\delta$ Standing Biomass $_{\mathrm{AD}}(\mathbf{g} \cdot \mathbf{m}-\mathbf{2})=(\mathrm{R} \delta$ Standing Biomss $X) X($ Avg Standing Biomass $)=(0.014) X(26.44)=\mathbf{0 . 5 2} \mathbf{g} \cdot \mathbf{m}-\mathbf{2}$ 


\section{Table 5}

\section{Estimation of Error in Daily Biomass Productivity}

Formula for calculating daily biomass productivity

Biomass Productivity (g.m-2.d-1) $=\left(\right.$ Standing Biomass $\mathrm{BD}_{\mathrm{D}+1}$ - Standing Biomass $\left.\mathrm{AD}_{\mathrm{D}}\right) / \mathrm{Day}$

Formulas for calculating error in daily biomass productivity

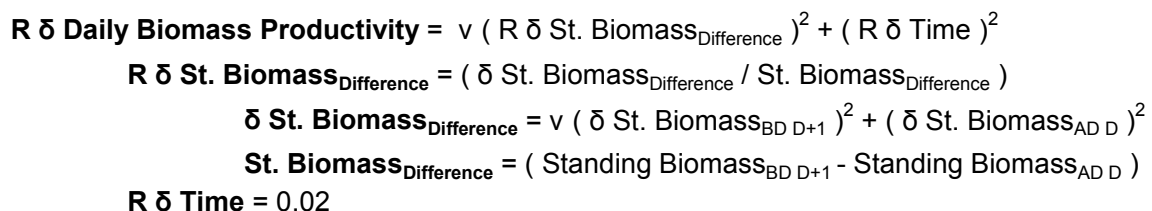

$\delta$ Daily Biomass Productivity (g.m-2.d-1) $=($ R $\delta$ Daily Biomass Productivity $) X($ Avg Daily Biomass Productivity $)$

Sample calculation of the R $\delta$ Daily Biomass Productivity

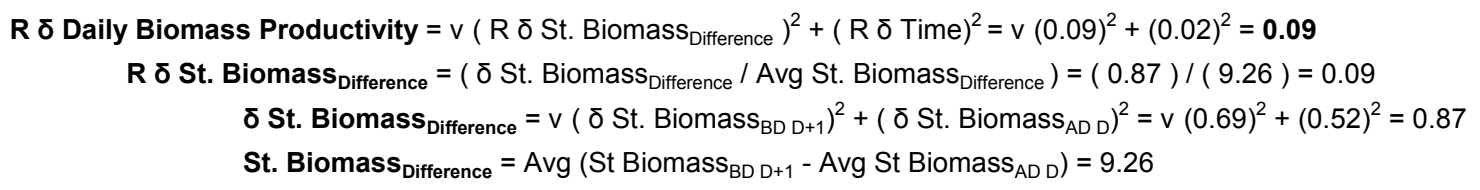

$\delta$ Daily Biomass Productivity (g.m-2.d-1) $=(R \delta$ Daily Biomas Productivity $) X($ Avg Daily Biomass Productivity $)=(0.09) X(9.6)=0.86$

Estimated error values used in calculating $\delta$ daily biomass productivity

\begin{tabular}{|c|c|}
\hline R $\delta$ St Biomass BD $=0.02$ & $m-2 . d-1)$ \\
\hline $\mathbf{R} \delta$ St Biomass BD $_{\mathrm{AD}}=0.02$ & $\delta$ \\
\hline
\end{tabular}

St. Biomass Bifference $(\mathbf{g} \cdot \mathbf{m}-2)=$ Avg $($ St. Biomass BD D+1 - St. Biomass BD D $)=9.26$

Avg Daily Biomass Productivity (g.m-2.d-1 = 9.6

R $\delta$ Time $=0.02$ 


\section{Table 6}

\section{Estimation of Error in Standing Biomass}

\section{Standing Biomas $\mathrm{S}_{\mathrm{BD}}$}

Example calculation being used is D5, Run 90 from Exp 16. Interpond consistancy

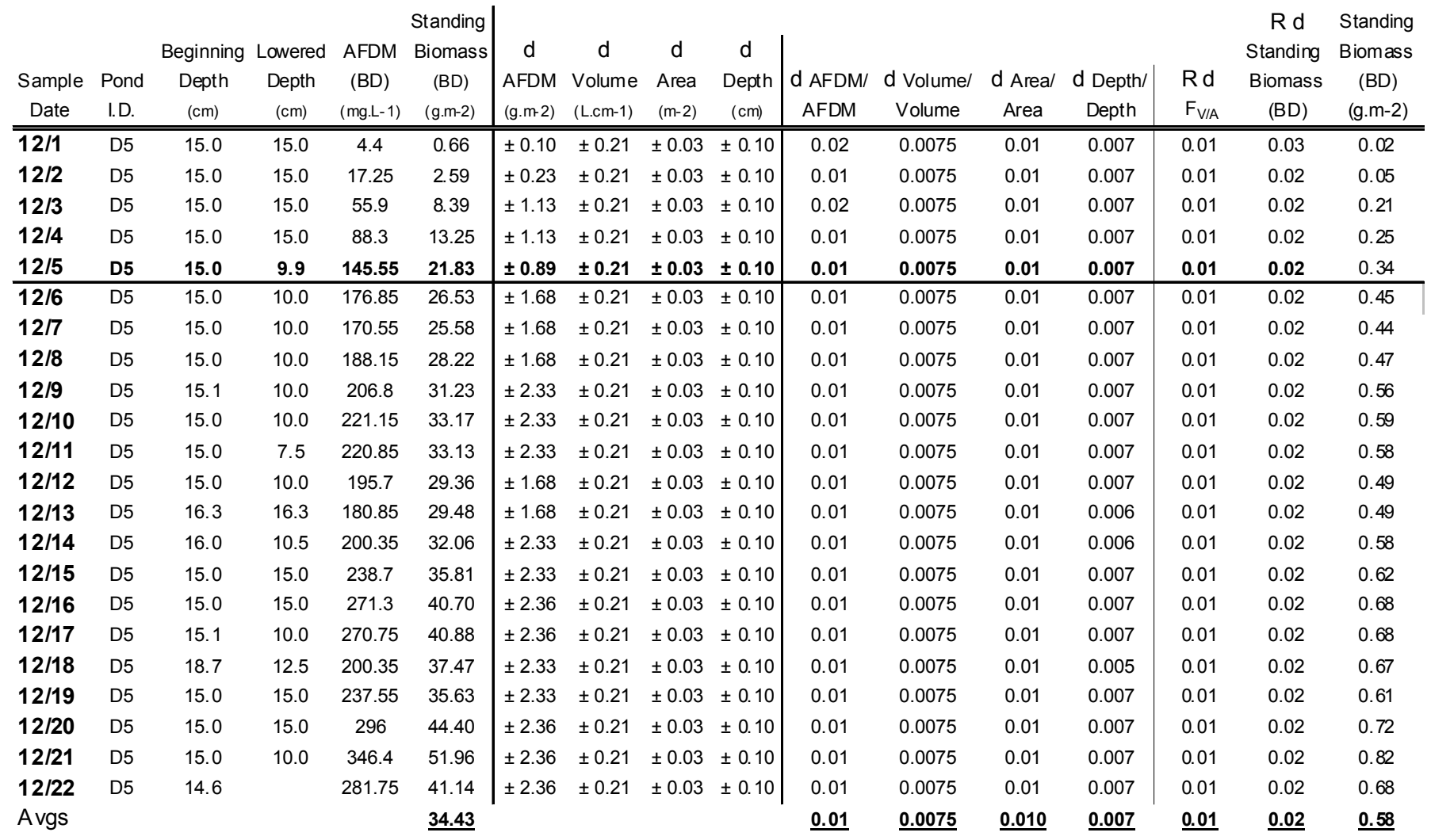


Table 7

\section{Estimation of Error in Standing Biomass}

Standing Biomass $\mathrm{SD}_{\mathrm{A}}$

Example calculation being used is D5, Run 90 from Exp 16. Interpond consistancy

\begin{tabular}{|c|c|c|c|c|c|c|c|c|c|c|c|c|c|c|c|c|}
\hline $\begin{array}{c}\text { Sample } \\
\text { Date }\end{array}$ & $\begin{array}{l}\text { Pond } \\
\text { I.D. }\end{array}$ & $\begin{array}{l}\text { Beginning } \\
\text { Depth } \\
(\mathrm{cm})\end{array}$ & $\begin{array}{l}\text { Lowered } \\
\text { Depth } \\
\text { (cm) }\end{array}$ & $\begin{array}{c}\text { AFDM } \\
\text { (BD) } \\
(\mathrm{mg} . \mathrm{L}-1)\end{array}$ & $\begin{array}{c}\text { Standing } \\
\text { Biomass } \\
(\mathrm{AD}) \\
(\mathrm{g} \cdot \mathrm{m}-2)\end{array}$ & $\begin{array}{c}\mathrm{d} \\
\text { AFDM } \\
\text { (g.m-2) }\end{array}$ & $\begin{array}{c}\mathrm{d} \\
\text { Volume } \\
\text { (L.cm-1) }\end{array}$ & $\begin{array}{c}\mathrm{d} \\
\text { Area } \\
(\mathrm{m}-2)\end{array}$ & $\begin{array}{c}\mathrm{d} \\
\text { Depth } \\
(\mathrm{cm})\end{array}$ & $\begin{array}{c}\text { d AFDM/ } \\
\text { AFDM }\end{array}$ & $\begin{array}{l}\text { d Volume/ } \\
\text { Volume }\end{array}$ & $\begin{array}{c}\text { d Area/ } \\
\text { Area }\end{array}$ & $\begin{array}{c}\text { d Depth/ } \\
\text { Depth }\end{array}$ & $\begin{array}{l}\mathrm{Rd} \\
\mathrm{F}_{\mathrm{V} / \mathrm{A}}\end{array}$ & $\begin{array}{c}\mathrm{R} \mathrm{d} \\
\text { Standing } \\
\text { Biomass } \\
\text { (AD) }\end{array}$ & $\begin{array}{c}\mathrm{d} \\
\text { Standing } \\
\text { Biomass } \\
\text { (AD) } \\
(\mathrm{g} \cdot \mathrm{m}-2)\end{array}$ \\
\hline$\overline{\overline{12 / 1}}$ & 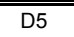 & "15.0 & $\begin{array}{c}15.0 \\
\end{array}$ & $\begin{array}{l}4.4 \\
\end{array}$ & "0.66 & $\bar{~} \pm 0.10$ & 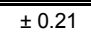 & $= \pm 0.03$ & 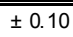 & "0.02 & 0.0075 & "0.01 & $\begin{array}{c}0.007 \\
\end{array}$ & 0.01 & "0.03 & 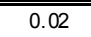 \\
\hline $12 / 2$ & D5 & 15.0 & 15.0 & 17.25 & 2.59 & \pm 0.23 & \pm 0.21 & \pm 0.03 & \pm 0.10 & 0.01 & 0.0075 & 0.01 & 0.007 & 0.01 & 0.02 & 0.05 \\
\hline $12 / 3$ & D5 & 15.0 & 15.0 & 55.9 & 8.39 & \pm 1.13 & \pm 0.21 & \pm 0.03 & \pm 0.10 & 0.02 & 0.0075 & 0.01 & 0.007 & 0.01 & 0.02 & 0.21 \\
\hline $12 / 4$ & D5 & 15.0 & 15.0 & 88.3 & 13.25 & \pm 1.13 & \pm 0.21 & \pm 0.03 & \pm 0.10 & 0.01 & 0.0075 & 0.01 & 0.007 & 0.01 & 0.02 & 0.25 \\
\hline $12 / 5$ & D5 & 15.0 & 9.9 & 145.55 & 14.41 & \pm 0.89 & \pm 0.21 & \pm 0.03 & \pm 0.10 & 0.01 & 0.0075 & 0.01 & 0.007 & 0.01 & 0.02 & 0.22 \\
\hline $12 / 6$ & D5 & 15.0 & 10.0 & 176.85 & 17.69 & \pm 1.68 & \pm 0.21 & \pm 0.03 & \pm 0.10 & 0.01 & 0.0075 & 0.01 & 0.007 & 0.01 & 0.02 & 0.30 \\
\hline $12 / 7$ & D5 & 15.0 & 10.0 & 170.55 & 17.06 & \pm 1.68 & \pm 0.21 & \pm 0.03 & \pm 0.10 & 0.01 & 0.0075 & 0.01 & 0.007 & 0.01 & 0.02 & 0.29 \\
\hline $12 / 8$ & D5 & 15.0 & 10.0 & 188.15 & 18.82 & \pm 1.68 & \pm 0.21 & \pm 0.03 & \pm 0.10 & 0.01 & 0.0075 & 0.01 & 0.007 & 0.01 & 0.02 & 0.31 \\
\hline $12 / 9$ & D5 & 15.1 & 10.0 & 206.8 & 20.68 & \pm 2.33 & \pm 0.21 & \pm 0.03 & \pm 0.10 & 0.01 & 0.0075 & 0.01 & 0.007 & 0.01 & 0.02 & 0.37 \\
\hline $12 / 10$ & D5 & 15.0 & 10.0 & 221.15 & 22.12 & \pm 2.33 & \pm 0.21 & \pm 0.03 & \pm 0.10 & 0.01 & 0.0075 & 0.01 & 0.007 & 0.01 & 0.02 & 0.39 \\
\hline $12 / 11$ & D5 & 15.0 & 7.5 & 220.85 & 16.56 & \pm 2.33 & \pm 0.21 & \pm 0.03 & \pm 0.10 & 0.01 & 0.0075 & 0.01 & 0.007 & 0.01 & 0.02 & 0.29 \\
\hline $12 / 12$ & D5 & 15.0 & 10.0 & 195.7 & 19.57 & \pm 1.68 & \pm 0.21 & \pm 0.03 & \pm 0.10 & 0.01 & 0.0075 & 0.01 & 0.007 & 0.01 & 0.02 & 0.32 \\
\hline $12 / 13$ & D5 & 16.3 & 16.3 & 180.85 & 29.48 & \pm 1.68 & \pm 0.21 & \pm 0.03 & \pm 0.10 & 0.01 & 0.0075 & 0.01 & 0.006 & 0.01 & 0.02 & 0.49 \\
\hline $12 / 14$ & D5 & 16.0 & 10.5 & 200.35 & 21.04 & \pm 2.33 & \pm 0.21 & \pm 0.03 & \pm 0.10 & 0.01 & 0.0075 & 0.01 & 0.006 & 0.01 & 0.02 & 0.38 \\
\hline $12 / 15$ & D5 & 15.0 & 15.0 & 238.7 & 35.81 & \pm 2.33 & \pm 0.21 & \pm 0.03 & \pm 0.10 & 0.01 & 0.0075 & 0.01 & 0.007 & 0.01 & 0.02 & 0.62 \\
\hline $12 / 16$ & D5 & 15.0 & 15.0 & 271.3 & 40.70 & \pm 2.36 & \pm 0.21 & \pm 0.03 & \pm 0.10 & 0.01 & 0.0075 & 0.01 & 0.007 & 0.01 & 0.02 & 0.68 \\
\hline $12 / 17$ & D5 & 15.1 & 10.0 & 270.75 & 27.08 & \pm 2.36 & \pm 0.21 & \pm 0.03 & \pm 0.10 & 0.01 & 0.0075 & 0.01 & 0.007 & 0.01 & 0.02 & 0.45 \\
\hline $12 / 18$ & D5 & 18.7 & 12.5 & 200.35 & 25.04 & \pm 2.33 & \pm 0.21 & \pm 0.03 & \pm 0.10 & 0.01 & 0.0075 & 0.01 & 0.005 & 0.01 & 0.02 & 0.45 \\
\hline $12 / 19$ & D5 & 15.0 & 15.0 & 237.55 & 35.63 & \pm 2.33 & \pm 0.21 & \pm 0.03 & \pm 0.10 & 0.01 & 0.0075 & 0.01 & 0.007 & 0.01 & 0.02 & 0.61 \\
\hline $12 / 20$ & D5 & 15.0 & 15.0 & 296 & 44.40 & \pm 2.36 & \pm 0.21 & \pm 0.03 & \pm 0.10 & 0.01 & 0.0075 & 0.01 & 0.007 & 0.01 & 0.02 & 0.72 \\
\hline $12 / 21$ & D5 & 15.0 & 10.0 & 346.4 & 34.64 & \pm 2.36 & \pm 0.21 & \pm 0.03 & \pm 0.10 & 0.01 & 0.0075 & 0.01 & 0.007 & 0.01 & 0.02 & 0.54 \\
\hline $12 / 22$ & D5 & 14.6 & & 281.75 & & \pm 2.36 & \pm 0.21 & \pm 0.03 & \pm 0.10 & 0.01 & 0.0075 & 0.01 & 0.007 & 0.01 & 0.02 & 0.00 \\
\hline Avgs & & & & & 26.44 & & & & & $\underline{0.01}$ & $\underline{0.0075}$ & $\underline{0.010}$ & $\underline{0.007}$ & $\underline{0.01}$ & $\underline{0.02}$ & $\underline{0.45}$ \\
\hline
\end{tabular}




\section{Table 8}

\section{Estimation of Error in Daily Biomass Productivity}

Biomass Productivity

Example calculation being used is D5, Run 90 from Exp 16. Interpond consistancy

\begin{tabular}{|c|c|c|c|c|c|c|c|c|c|c|c|c|c|}
\hline $\begin{array}{c}\text { Sample } \\
\text { Date }\end{array}$ & $\begin{array}{l}\text { Pond } \\
\text { I.D. }\end{array}$ & $\begin{array}{c}\text { Age } \\
\text { (Days) }\end{array}$ & $\begin{array}{c}\text { AFDM } \\
(\mathrm{BD}) \\
(\mathrm{mg} . \mathrm{L}-1)\end{array}$ & $\begin{array}{c}\text { Standing } \\
\text { Biomass } \\
\text { (BD) } \\
\text { (g.m-2) }\end{array}$ & $\begin{array}{c}\mathrm{d} \\
\text { Standing } \\
\text { Biomass } \\
(\mathrm{BD}) \\
(\mathrm{g} \cdot \mathrm{m}-2)\end{array}$ & $\begin{array}{c}\text { Standing } \\
\text { Biomass } \\
\text { (AD) } \\
\text { (g.m-2) }\end{array}$ & $\begin{array}{c}\mathrm{d} \\
\text { Standing } \\
\text { Biomass } \\
(\mathrm{AD}) \\
(\mathrm{g} \cdot \mathrm{m}-2)\end{array}$ & $\begin{array}{c}\text { Difference } \\
\text { in } \\
\text { Standing } \\
\text { Biomass } \\
\text { (g.m-2) }\end{array}$ & $\begin{array}{c}d \\
\text { Difference } \\
\text { in } \\
\text { Standing } \\
\text { Biomass } \\
\text { (g.m-2) }\end{array}$ & $\begin{array}{c}\mathrm{R} \mathrm{d} \\
\text { Difference } \\
\text { in } \\
\text { Standing } \\
\text { Biomass }\end{array}$ & $\begin{array}{l}\mathrm{Rd} \\
\text { Time }\end{array}$ & $\begin{array}{c}\text { R d } \\
\text { Daily } \\
\text { Biomass } \\
\text { Productivity } \\
\text { (g.m-2) }\end{array}$ & $\begin{array}{c}\mathrm{d} \\
\text { Daily } \\
\text { Biomass } \\
\text { Productivity } \\
\text { (g.m-2.d-1) }\end{array}$ \\
\hline $12 / 1$ & D5 & 0 & 4.4 & 0.66 & 0.02 & 0.66 & 0.02 & 1.93 & 0.05 & 0.03 & 0.02 & 0.03 & 0.07 \\
\hline $12 / 2$ & D5 & 1 & 17.25 & 2.59 & 0.05 & 2.59 & 0.05 & 5.80 & 0.21 & 0.04 & 0.02 & 0.04 & 0.24 \\
\hline $12 / 3$ & D5 & 2 & 55.9 & 8.39 & 0.21 & 8.39 & 0.21 & 4.86 & 0.33 & 0.07 & 0.02 & 0.07 & 0.34 \\
\hline $12 / 4$ & D5 & 3 & 88.3 & 13.25 & 0.25 & 13.25 & 0.25 & 8.59 & 0.42 & 0.05 & 0.02 & 0.05 & 0.45 \\
\hline $12 / 5$ & D5 & 4 & 145.55 & 21.83 & 0.34 & 14.41 & 0.22 & 12.12 & 0.50 & 0.04 & 0.02 & 0.05 & 0.56 \\
\hline $12 / 6$ & D5 & 5 & 176.85 & 26.53 & 0.45 & 17.69 & 0.30 & 7.90 & 0.53 & 0.07 & 0.02 & 0.07 & 0.56 \\
\hline $12 / 7$ & D5 & 6 & 170.55 & 25.58 & 0.44 & 17.06 & 0.29 & 11.17 & 0.56 & 0.05 & 0.02 & 0.05 & 0.60 \\
\hline $12 / 8$ & D5 & 7 & 188.15 & 28.22 & 0.47 & 18.82 & 0.31 & 12.41 & 0.65 & 0.05 & 0.02 & 0.06 & 0.69 \\
\hline $12 / 9$ & D5 & 8 & 206.8 & 31.23 & 0.56 & 20.68 & 0.37 & 12.49 & 0.69 & 0.06 & 0.02 & 0.06 & 0.74 \\
\hline $12 / 10$ & D5 & 9 & 221.15 & 33.17 & 0.59 & 2212 & 0.39 & 11.01 & 0.70 & 0.06 & 0.02 & 0.07 & 0.74 \\
\hline $12 / 11$ & D5 & 10 & 220.85 & 33.13 & 0.58 & 16.56 & 0.29 & 12.79 & 0.57 & 0.04 & 0.02 & 0.05 & 0.62 \\
\hline $12 / 12$ & D5 & 11 & 195.7 & 29.36 & 0.49 & 19.57 & 0.32 & 9.91 & 0.59 & 0.06 & 0.02 & 0.06 & 0.62 \\
\hline $12 / 13$ & D5 & 12 & 180.85 & 29.48 & 0.49 & 29.48 & 0.49 & 2.58 & 0.76 & 0.30 & 0.02 & 0.30 & 0.76 \\
\hline $12 / 14$ & D5 & 13 & 200.35 & 32.06 & 0.58 & 21.04 & 0.38 & 14.77 & 0.72 & 0.05 & 0.02 & 0.05 & 0.78 \\
\hline $12 / 15$ & D5 & 14 & 238.7 & 35.81 & 0.62 & 35.81 & 0.62 & 4.89 & 0.91 & 0.19 & 0.02 & 0.19 & 0.92 \\
\hline $12 / 16$ & D5 & 15 & 271.3 & 40.70 & 0.68 & 40.70 & 0.68 & 0.19 & 0.96 & 5.09 & 0.02 & 5.09 & 0.96 \\
\hline $12 / 17$ & D5 & 16 & 270.75 & 40.88 & 0.68 & 27.08 & 0.45 & 10.39 & 0.81 & 0.08 & 0.02 & 0.08 & 0.83 \\
\hline $12 / 18$ & D5 & 17 & 200.35 & 37.47 & 0.67 & 25.04 & 0.45 & 10.59 & 0.76 & 0.07 & 0.02 & 0.07 & 0.79 \\
\hline $12 / 19$ & D5 & 18 & 237.55 & 35.63 & 0.61 & 35.63 & 0.61 & 8.77 & 0.95 & 0.11 & 0.02 & 0.11 & 0.96 \\
\hline $12 / 20$ & D5 & 19 & 296 & 44.40 & 0.72 & 44.40 & 0.72 & 7.56 & 1.09 & 0.14 & 0.02 & 0.15 & 1.10 \\
\hline $12 / 21$ & D5 & 20 & 346.4 & 51.96 & 0.82 & 34.64 & 0.54 & 6.50 & 0.87 & 0.13 & 0.02 & 0.14 & 0.88 \\
\hline $12 / 22$ & D5 & 21 & 281.75 & 41.14 & 0.68 & & 0.00 & & & & & & \\
\hline Avgs & & & & & & & & 9.26 & 0.76 & 0.41 & 0.02 & 0.43 & $\underline{0.78}$ \\
\hline
\end{tabular}




\section{Table 9}

\section{Estimation of Error in Daily Biomass Productivity}

Averages for all Runs in Reproducibility Experiment.

\begin{tabular}{cc}
$\begin{array}{c}\text { Productivity } \\
\text { Range } \\
\text { (g.m-2.d-1) }\end{array}$ & $\begin{array}{c}\mathrm{d} \\
\text { Productivity } \\
\text { (g.m-2.d-1) }\end{array}$ \\
\hline $0.0-0.99$ & 1.00 \\
$1.0-1.99$ & 0.08 \\
$2.0-2.99$ & 0.92 \\
$3.0-3.99$ & 0.99 \\
$4.0-4.99$ & 0.75 \\
$5.0-5.99$ & 0.58 \\
$6.0-6.99$ & 0.81 \\
$7.0-7.99$ & 0.76 \\
$8.0-8.99$ & 0.66 \\
$9.0-9.99$ & 0.85 \\
$10.0-10.99$ & 0.77 \\
$11.0-11.99$ & 0.78 \\
$12.0-12.99$ & 0.72 \\
$13.0-13.99$ & 0.79 \\
$14.0-14.99$ & 0.77 \\
$15.0-15.99$ & 0.82
\end{tabular}




\subsubsection{Reproducibility of Results}

Although the reproducibility of the experimental apparatus was checked at the initial startup of the Phase 1 project, a detailed analysis was performed during the Phase 2 project. Two trials were run, one during the winter and the other in the spring. The four experimental open raceway ponds were operated in the same way, the biomass productivity was measured, and the results compared using paired t-tests.

As mentioned above, there is day to day variability in measured biomass productivity from each pond due to variable inputs of weather. That makes it irrelevant to compare the means of biomass production over a given number of days of the four ponds to each other. Rather it is appropriate to ask whether, between each pair of ponds, the mean of the difference in the daily biomass productivity measured for each pond is different from zero. This test is called a paired t-test, because productivities are only compared on the same day not on different days.

The four cultures were operated at the same $\mathrm{pH}$ setting (8.1), the same depth $(15 \mathrm{~cm})$, the same dilution rate ( $5 \mathrm{~cm} . \mathrm{d}-1$, semi continuously), the same nutrient input (sufficiency) and inoculated with the same amount from one inoculation culture. The results are summarized in Table 10 and, for one of the trials, shown graphically in Figure 2. The listing of the daily productivities and the means are given in Table 11. There are some obvious differences in biomass productivity on a few days, but reproducibility among the units was very good overall. The data on one day (12/06) was left out because the CO2 inlet valve to one of the ponds was stuck closed on that day. The biomass productivity in that pond on that day was less than half of the productivities from the other ponds. On two other days there were noticeable differences in production from the same pond. Since there were no anomalies attributable to operating parameters, these days were included in the statistical analysis. As shown in Table 12, to the 5\% confidence level, there were no pairs of ponds which showed means of differences in daily productivities that were other than zero, i.e., t-stat was less than t-critical in all cases.

Combining these results with the results of the error propagation analysis, we are quite confident that an experimental result which shows a statistical difference between treatments is real. Of course, statistically significant differences are not necessarily of practical importance.

It must be noted, however, that it is not uncommon for one or another culture in replicate cultures to lose density, to a greater degree or sooner, than the others over the period of operation. The response of algal cells to inputs such as light and temperature, is highly nonlinear. Thus large divergences may occur due to relatively small, even undetectable, differences in treatment or in the physiological state of the cells. In addition, invasion of the cultures by other species (predators in particular, but also possibly viruses, fungi, lytic bacteria, etc.) is another factor which can result in inconsistent results between replicates. 
Table 10. Summary of Reproducibility Experiments.

\begin{tabular}{|c|c|c|c|c|c|c|c|}
\hline \multirow{2}{*}{$\begin{array}{l}\text { Run } \\
\text { No. }\end{array}$} & \multirow{2}{*}{$\begin{array}{c}\text { Avg } \\
\text { AFDM } \\
\text { (mg.L- } \\
\text { 1) }\end{array}$} & \multirow[b]{2}{*}{ StDev } & \multirow{2}{*}{$\begin{array}{c}\text { Max } \\
\text { Biomass } \\
\text { Prod. } \\
\text { (g.m- } \\
\text { 2.d-1) }\end{array}$} & \multirow{2}{*}{$\begin{array}{c}\text { Avg } \\
\text { Biomass } \\
\text { Prod. } \\
\text { (g.m- } \\
\text { 2.d-1) }\end{array}$} & \multicolumn{3}{|c|}{$\begin{array}{c}\text { Avg } \\
\text { Photo. } \\
\text { Efficiency }\end{array}$} \\
\hline & & & & & StDev & $(\%)$ & StDev \\
\hline 90 & 224 & \pm 51 & 14.8 & 9.3 & \pm 4.0 & 3.9 & \pm 2.1 \\
\hline 91 & 251 & \pm 49 & 15.3 & 9.7 & \pm 4.2 & 4.1 & \pm 2.0 \\
\hline 92 & 250 & \pm 48 & 15.4 & 9.4 & \pm 4.4 & 3.9 & \pm 2.0 \\
\hline 93 & 242 & \pm 55 & 15.2 & 9.6 & \pm 4.0 & 4.0 & \pm 1.7 \\
\hline
\end{tabular}

Cyclotella, strain J5, Dates of averages 12/5 - 12/21

Average daily insolation $=3351 \mathrm{w} . \mathrm{hr} \cdot \mathrm{m}-\mathbf{2} . \mathrm{d}-1$

Average max/min pond temperatures: 24/14 C

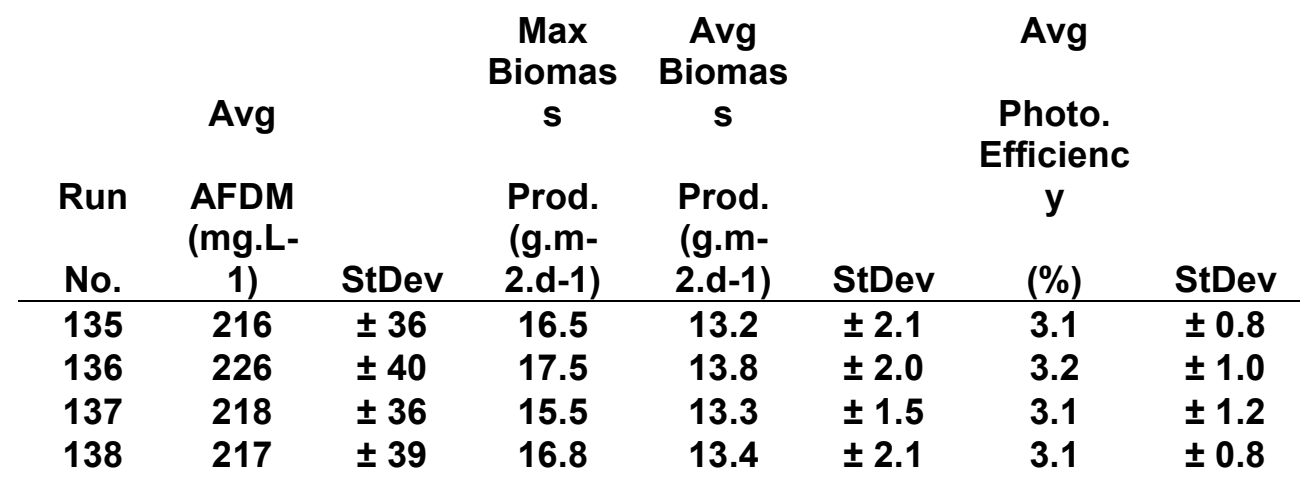

Cyclotella, strain J5, Dates of averages 4/26-5/2

Average daily insolation $=6106 \mathrm{w} \cdot \mathrm{hr} \cdot \mathrm{m}-\mathbf{2} \cdot \mathrm{d}-1$

Average max/min pond temperatures: $30 / 17 \mathrm{C}$ 
Figure 2. Interpond Production Consistancy of Cyclotella sp (strain Cyclo J) in Small Ponds, Semi-continuous Culture.

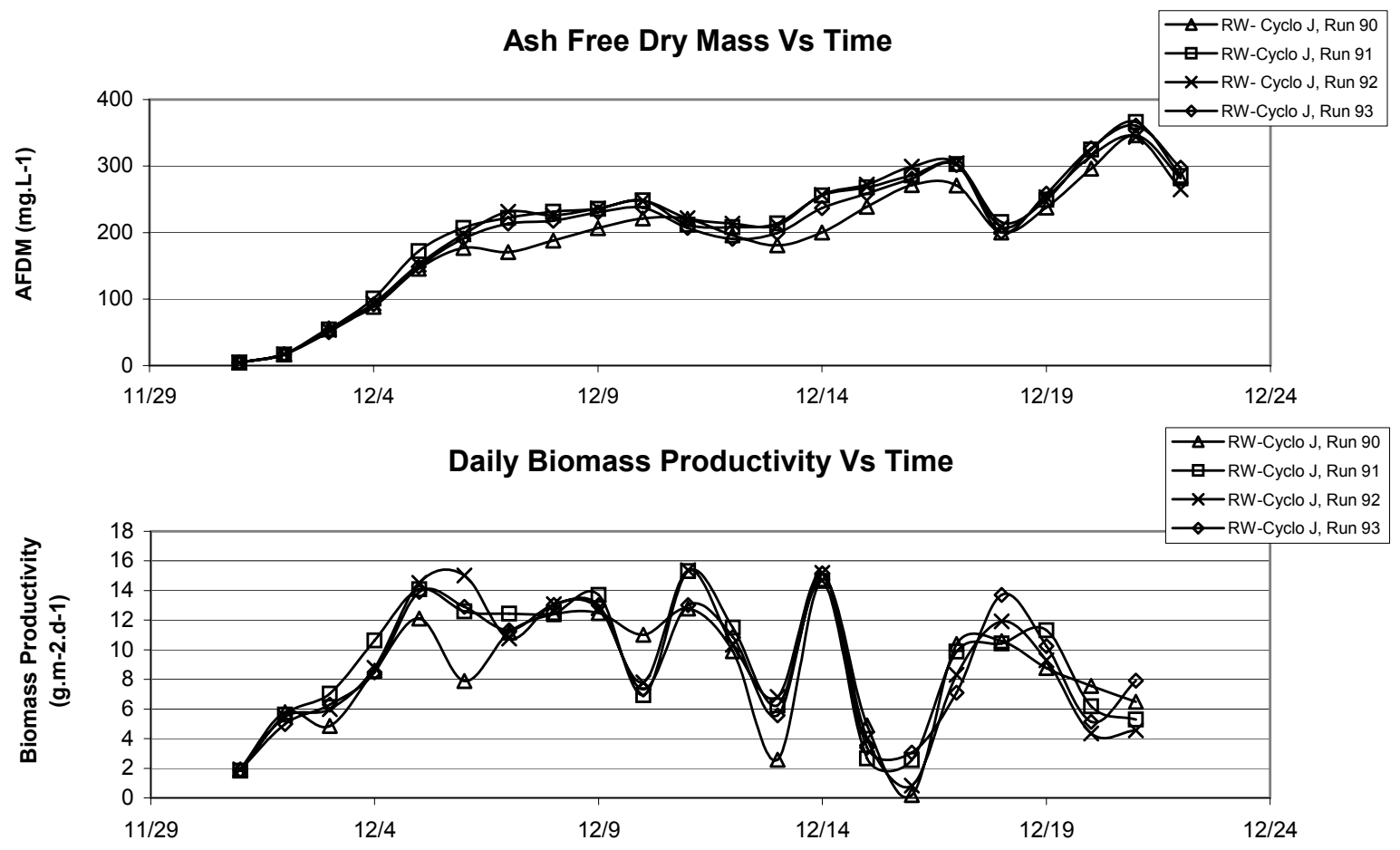

Pond Water Temperature Vs Time

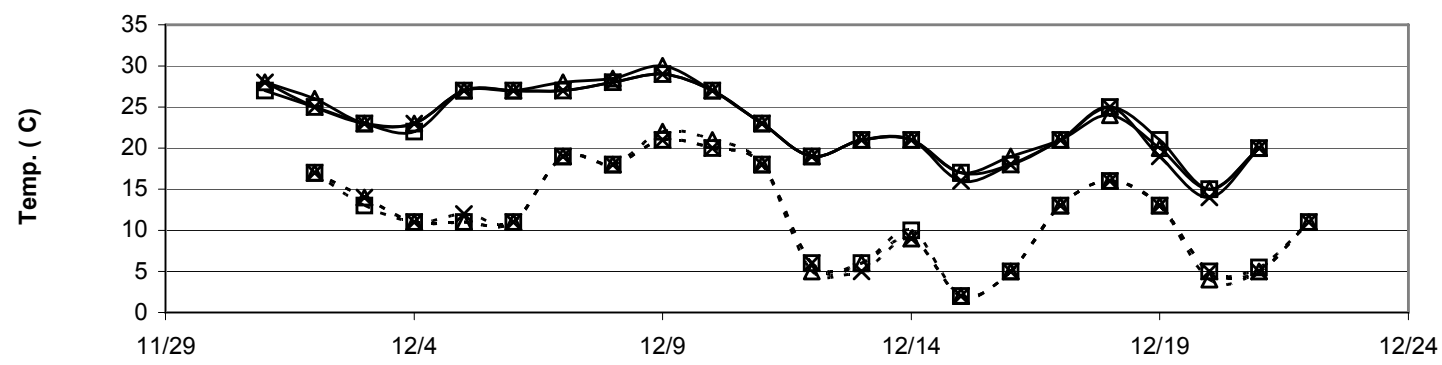

Total Daily Insolation \&

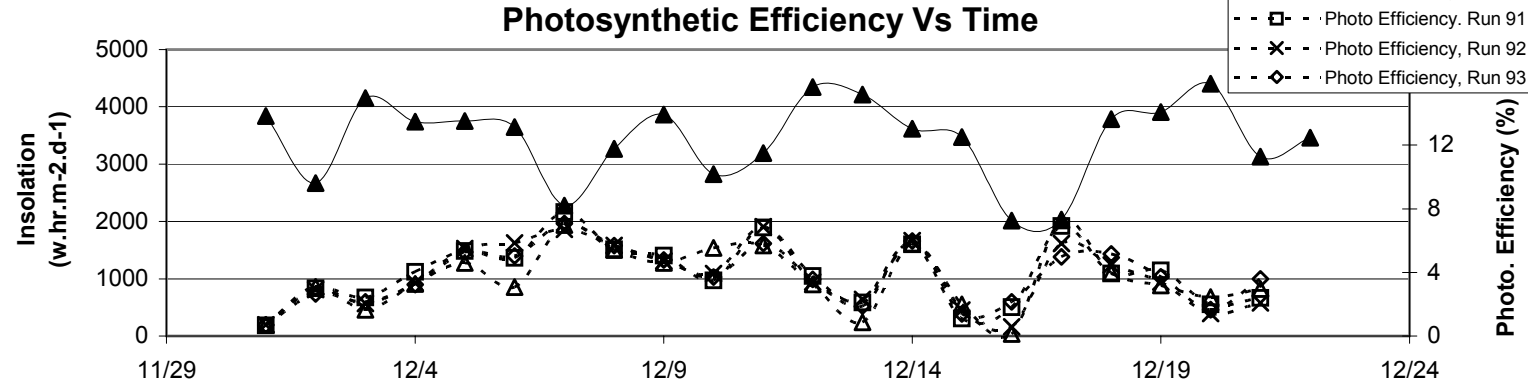


Table 11. Daily Productivities (g/m2/d) in Reproducibility Experiments.

\begin{tabular}{|c|c|c|c|c|c|c|c|c|c|}
\hline Date & $\begin{array}{c}\text { D5 } \\
\text { Run } \\
90\end{array}$ & $\begin{array}{c}\text { D1 } \\
\text { Run } \\
91\end{array}$ & $\begin{array}{c}\text { D2 } \\
\text { Run } \\
92\end{array}$ & $\begin{array}{c}\text { D3 } \\
\text { Run } \\
93\end{array}$ & Dates & $\begin{array}{l}\text { D5 } \\
\text { Run } \\
135\end{array}$ & $\begin{array}{c}\text { D1 } \\
\text { Run } \\
136\end{array}$ & $\begin{array}{c}\text { D2 } \\
\text { Run } \\
137\end{array}$ & $\begin{array}{c}\text { D3 } \\
\text { Run } \\
138\end{array}$ \\
\hline $12 / 5$ & 12.1 & 14.1 & 14.5 & 13.9 & $4 / 26$ & 14.9 & 14.9 & 13.2 & 14.4 \\
\hline $12 / 7$ & 11.2 & 12.5 & 10.8 & 11.3 & $4 / 27$ & 11.4 & 11.4 & 11.5 & 11.5 \\
\hline $12 / 8$ & 12.4 & 12.5 & 13.1 & 13.0 & $4 / 28$ & 12.2 & 13.2 & 12.8 & 12.6 \\
\hline $12 / 9$ & 12.5 & 13.7 & 12.8 & 13.0 & $4 / 29$ & 12.8 & 12.7 & 11.9 & 12.9 \\
\hline $12 / 10$ & 11.0 & 6.9 & 7.8 & 7.3 & $4 / 30$ & 16.5 & 17.5 & 15.5 & 16.8 \\
\hline $12 / 11$ & 12.8 & 15.3 & 15.4 & 13.0 & $5 / 1$ & 10.4 & 12.7 & 13.4 & 10.6 \\
\hline $12 / 12$ & 9.9 & 11.5 & 10.3 & 10.9 & $5 / 2$ & 14.1 & 13.9 & 15.0 & 14.8 \\
\hline $12 / 13$ & 2.6 & 6.3 & 6.8 & 5.6 & & & & & \\
\hline $12 / 14$ & 14.8 & 14.7 & 15.2 & 15.2 & & & & & \\
\hline $12 / 15$ & 4.9 & 2.7 & 4.0 & 3.4 & & & & & \\
\hline $12 / 16$ & 0.2 & 2.6 & 0.8 & 3.1 & & & & & \\
\hline $12 / 17$ & 10.4 & 9.9 & 8.3 & 7.1 & & & & & \\
\hline $12 / 18$ & 10.6 & 10.4 & 11.9 & 13.7 & & & & & \\
\hline $12 / 19$ & 8.8 & 11.3 & 9.3 & 10.3 & & & & & \\
\hline $12 / 20$ & 7.6 & 6.2 & 4.4 & 5.1 & & & & & \\
\hline $12 / 21$ & 6.5 & 5.3 & 4.6 & 7.9 & & & & & \\
\hline & 9.3 & 9.7 & 9.4 & 9.6 & Avg & 13.2 & 13 & 13.3 & \\
\hline
\end{tabular}


Table 12. Statistical analysis summary table for Reproducibility among ponds. Confidence Interval $=95 \%$

\begin{tabular}{|c|c|c|c|c|c|c|c|}
\hline $\begin{array}{c}\text { Run } \\
\#\end{array}$ & $\begin{array}{c}\text { Avg. } \\
\text { Biomass } \\
\text { Productivity } \\
\text { (g.m-2.d-1) }\end{array}$ & $\begin{array}{c}\text { t-Stat } \\
\text { (one- } \\
\text { tail) }\end{array}$ & $\begin{array}{c}\text { t- } \\
\text { Critical } \\
\text { (one- } \\
\text { tail) }\end{array}$ & $\begin{array}{c}\text { Run } \\
\#\end{array}$ & $\begin{array}{c}\text { Avg. } \\
\text { Biomass } \\
\text { Productivity } \\
\text { (g.m-2.d-1) }\end{array}$ & $\begin{array}{c}\text { t-Stat } \\
\text { (one- } \\
\text { tail) }\end{array}$ & $\begin{array}{c}\text { t- } \\
\text { Critical } \\
\text { (one- } \\
\text { tail) }\end{array}$ \\
\hline 90 & 9.3 & \multirow{2}{*}{0.9} & \multirow{2}{*}{1.8} & 136 & 13.8 & 1.7 & 1.9 \\
\hline 91 & 9.7 & & & 137 & 13.3 & & \\
\hline 91 & 9.7 & \multirow{2}{*}{1.2} & \multirow{2}{*}{1.8} & 136 & 13.8 & 0.9 & 1.9 \\
\hline 92 & 9.4 & & & 138 & 13.4 & & \\
\hline 91 & 9.7 & \multirow{2}{*}{0.3} & \multirow{2}{*}{1.8} & 136 & 13.8 & 1.1 & 1.9 \\
\hline 93 & 9.6 & & & 135 & 13.2 & & \\
\hline 90 & 9.3 & \multirow{2}{*}{0.2} & \multirow{2}{*}{1.8} & 137 & 13.3 & 0.1 & 1.9 \\
\hline 92 & 9.4 & & & 138 & 13.4 & & \\
\hline 92 & 9.4 & \multirow{2}{*}{0.7} & \multirow{2}{*}{1.8} & 137 & 13.3 & 0.3 & 1.9 \\
\hline 93 & 9.6 & & & 135 & 13.2 & & \\
\hline 93 & 9.6 & \multirow{2}{*}{0.7} & \multirow{2}{*}{1.8} & 138 & 13.4 & 1.4 & 1.9 \\
\hline 90 & 9.3 & & & 135 & 13.2 & & \\
\hline
\end{tabular}




\subsection{Media Optimization}

\subsubsection{Silicon Nutrition}

In order to study the role of available light in the production of biomass, it is necessary for light to be the nutrient limiting the biomass productivity. Thus other nutrients, such as silicon must be supplied in excess. Other parameters, such as $\mathrm{pH}$, must also be optimal. Experiments were performed to demonstrate that light was indeed the only limiting factor.

Silicon is an essential nutrient for the formation of the frustule (skeleton) of diatoms. The saline groundwater used provides, on average, about $5 \mathrm{mg} / \mathrm{L} \mathrm{Si}$, which is equivalent to 0.35 g.m-2.d-1 of Si for ponds operated at $15 \mathrm{~cm}$ depth, diluted 33\% per day. Silicates (oxides of $\mathrm{Si}$ ) are only moderately soluble in seawater in theory, and quite difficult to solubolize in practice. If added too quickly, or without adequate mixing, the silicates precipitate, causing the algae to flocculate. Flocs are nucleation sites for cell debris, bacteria, and protozoan. They are highly correlated with incipient collapse of the culture. For this reason, the amount of silicate added is kept as close as possible to the amount required. How much is actually required? The literature reports silica content of diatoms at typically $20 \%$ by weight, although it is unclear whether this refers to $\mathrm{Si}(\mathrm{MW}=28.3)$ or $\mathrm{SiO} 2(\mathrm{MW}=60.3)$. During the winter months, Si was added as if the cell quota for Si were 0.1, i.e., sufficient to grow about ten times its weight in algal biomass. Experiments were performed throughout the year to determine whether Si was limiting the biomass production of the cultures. In the typical experiment, two ponds were operated in parallel, one with a higher daily addition of Si.

The first trial was run from mid February to mid April. Temperature was increasing, but still cool compared to the optimal growth temperatures of the late spring and summer when morning temperatures climb above $25 \mathrm{C}$ by $9 \mathrm{AM}$, and afternoon temperatures are above 30 C. Insolation was about $60 \%$ of summertime insolation. Another trial was run in May when temperature is optimal and insolation high. A third trial was run in July when temperatures are higher yet, but insolation is lower due to cloud cover. Results are shown in Table 13 and Figure 3.

For the low Si culture in trial 1, the added Si and the Si in the saline groundwater were sufficient to support about $14 \mathrm{~g} / \mathrm{m} 2 / \mathrm{d}$ of biomass production at a cell quota of $10 \%$. In the second culture almost twice the Si was added. As shown in the table and figure, there was no difference between the two treatments in terms of biomass production. This suggested that the biomass production was light or temperature limited, not Si limited. Although maximal biomass production was higher, it is an uncertain measure of nutrient limitation, since Si can be depleted to some extent internally on a short term basis, without reducing biomass production.

During the second trial (Figure 3b) the average biomass production was over 35\% greater in the culture supplemented with more Si relative to the low Si culture. Even though the insolation was 50\% higher, and the temperatures were more optimal, the biomass production from the low Si treatment was hardly any different than in trial 1. It thus is reasonable to conclude that Si was limiting the biomass production. 
With this demonstrated, the next step was to further increase the Si in the high Si treatment (Table 13 and Figure 3c). At first, the higher Si led to even greater biomass production. This was, however, not long lasting. The high Si treatment led to increasing clumping in the culture. Therefore, optimal Si supply will have to be metered in over the day. Comparing the low Si culture in this trial to the low Si culture in the second trial confirms that the latter was Si limited. That is, increasing Si input increased productivity. Comparing the two cultures in this third trial indicates that 2.2 g.m-2 of Si was sufficient to support 20 g.m-2.d1. Adding more Si did not, on average, increase biomass production.

These experiments did provide information on what the Si cell quotient for this diatom is. Assuming that the residual Si level in the culture medium after growth was reduced by the algal cells down to very low levels (which is invariably true when a nutrient is limiting or nearly limiting), it took about $2.2-2.3 \mathrm{~g}$ Si to produce $20 \mathrm{~g}$ of fully-sufficient algal biomass.

\subsection{2. pH Experiment.}

A pH of 8 was used for most of the experiments performed. This value was chosen to limit the loss of $\mathrm{CO} 2$ from open pond cultures due to out gassing. At this $\mathrm{pH}$, in seawater, the driving force for loss of carbon dioxide to the atmosphere is relatively low. However, many microalgae grow somewhat better at lower $\mathrm{pH}$. Thus two ponds were operated with the same Cyclotella culture, one at $\mathrm{pH} 8$ and one at $\mathrm{pH} 7.5$, to establish that at $\mathrm{pH}$ 8, biomass productivity is not limited. This experiment was carried out during the summer, when biomass productivity would be expected to be maximal.

Results are given in Table 14. There was no statistical difference between the biomass productivities at the two vales of $\mathrm{pH}$. 
Table 13. Summary of Si Sufficiency Experiments

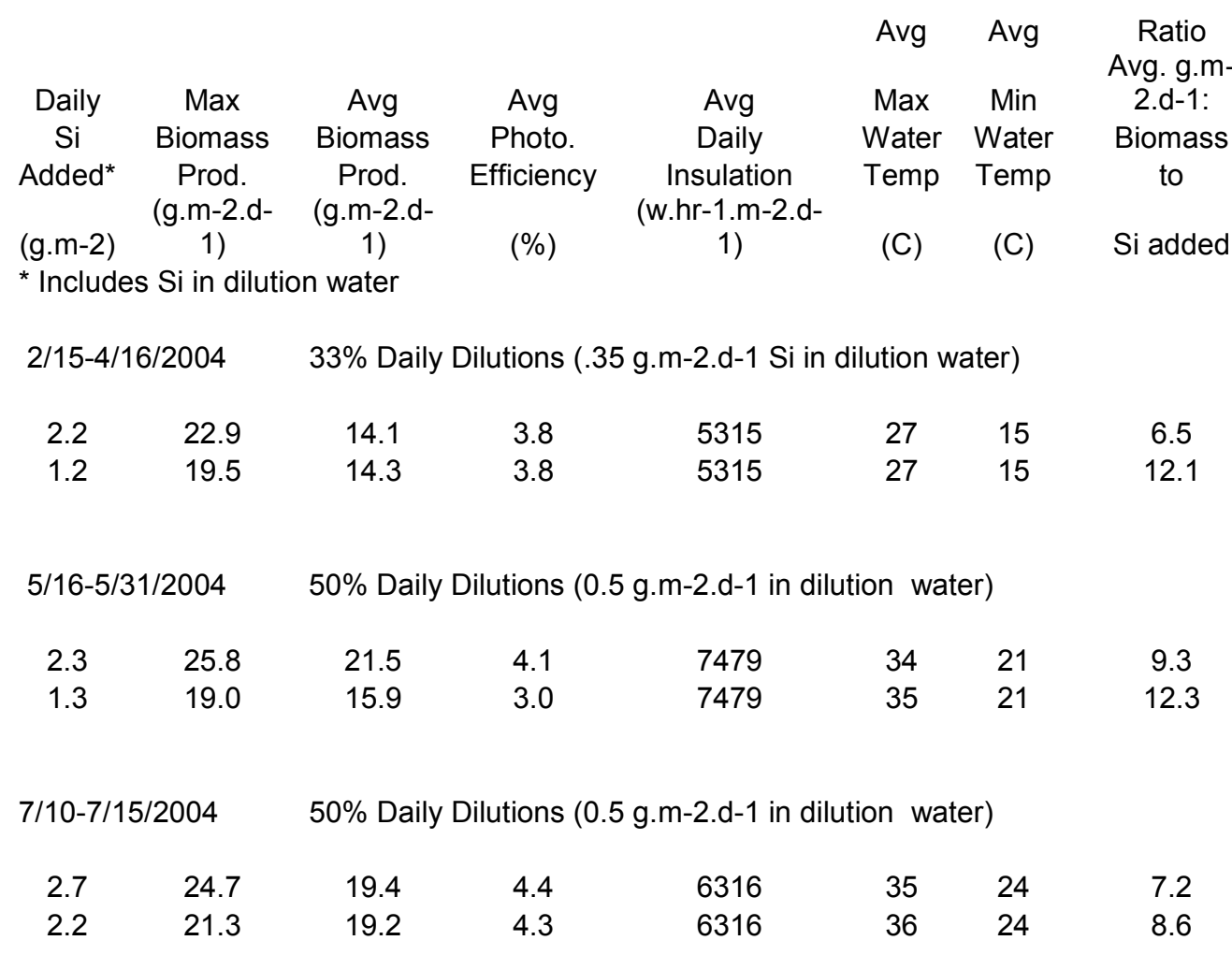


Figure 3. Si Addition and Productivity- Comparison of Three Experiments

Figure 3a. Trial 1: High vs Low Silicate Feeding Cyclotella sp (strain Cyclo-J) in Small Pond, Semi-continuous Culture. (2.2 g.m-2 vs 1.2 g.m-2 Si added)
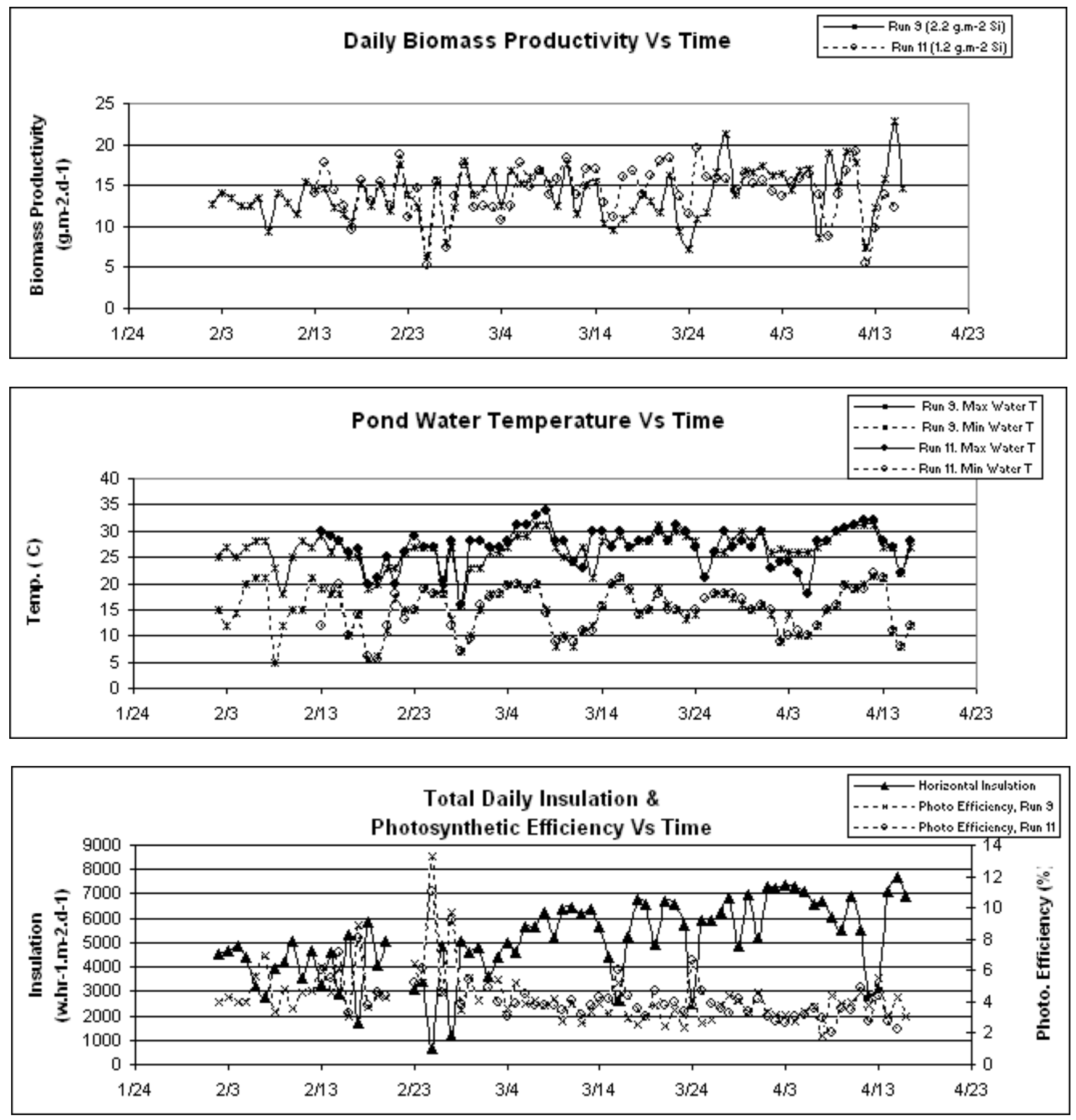
Figure 3b. Trial 2: High vs Low Silicate Feeding Cyclotella sp (strain- Cyclo-J) in Small Pond, Semi-continuous Culture ( $50 \%$ dilution).
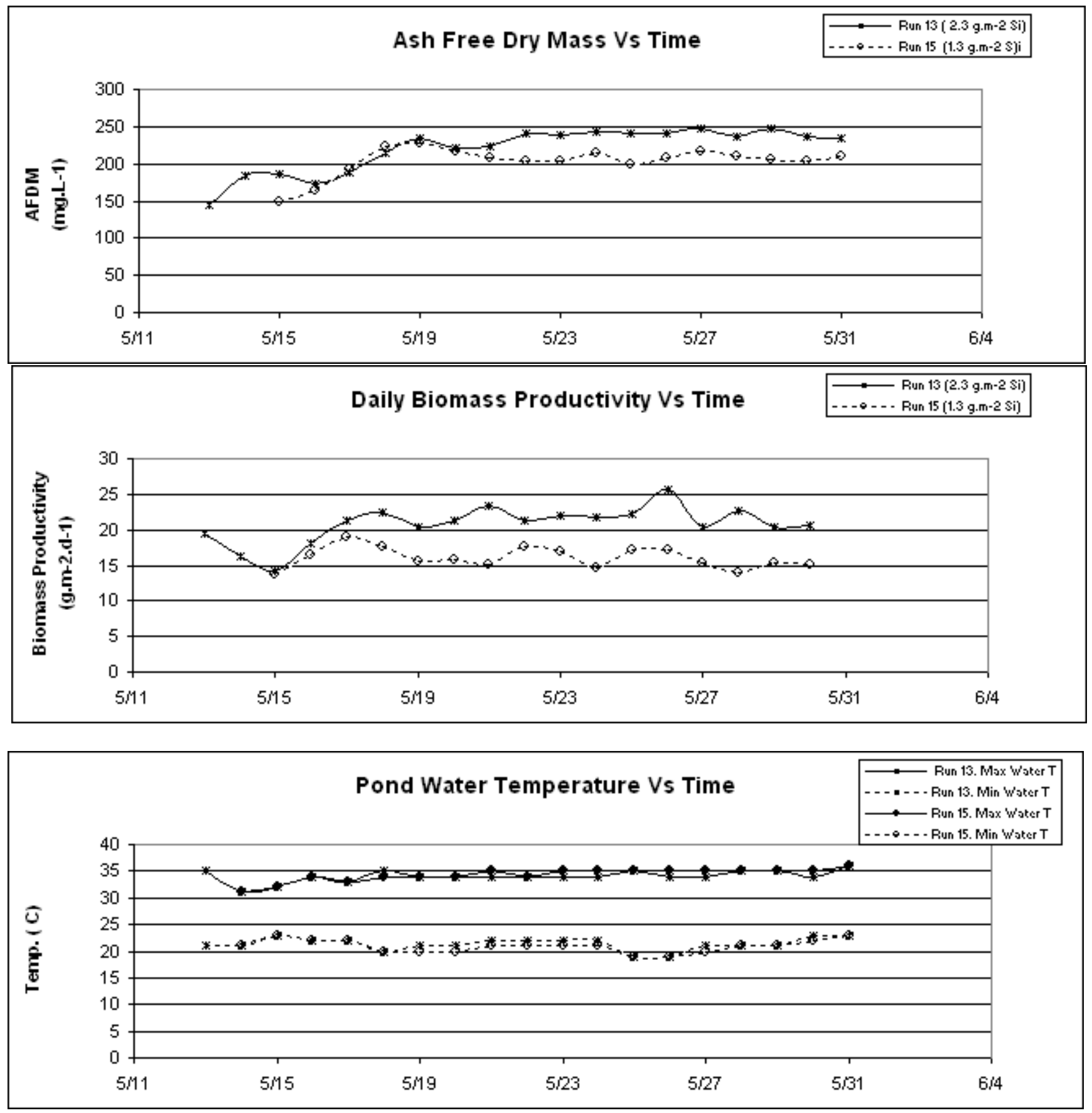
Figure 3c. Trial 3: High vs Low Silicate Feeding Cyclotella sp (strain- Cyclo-J) in Small Pond, in Semi-continuous Culture. (2.7 g.m-2 v 2.2 g.m-2 Si )
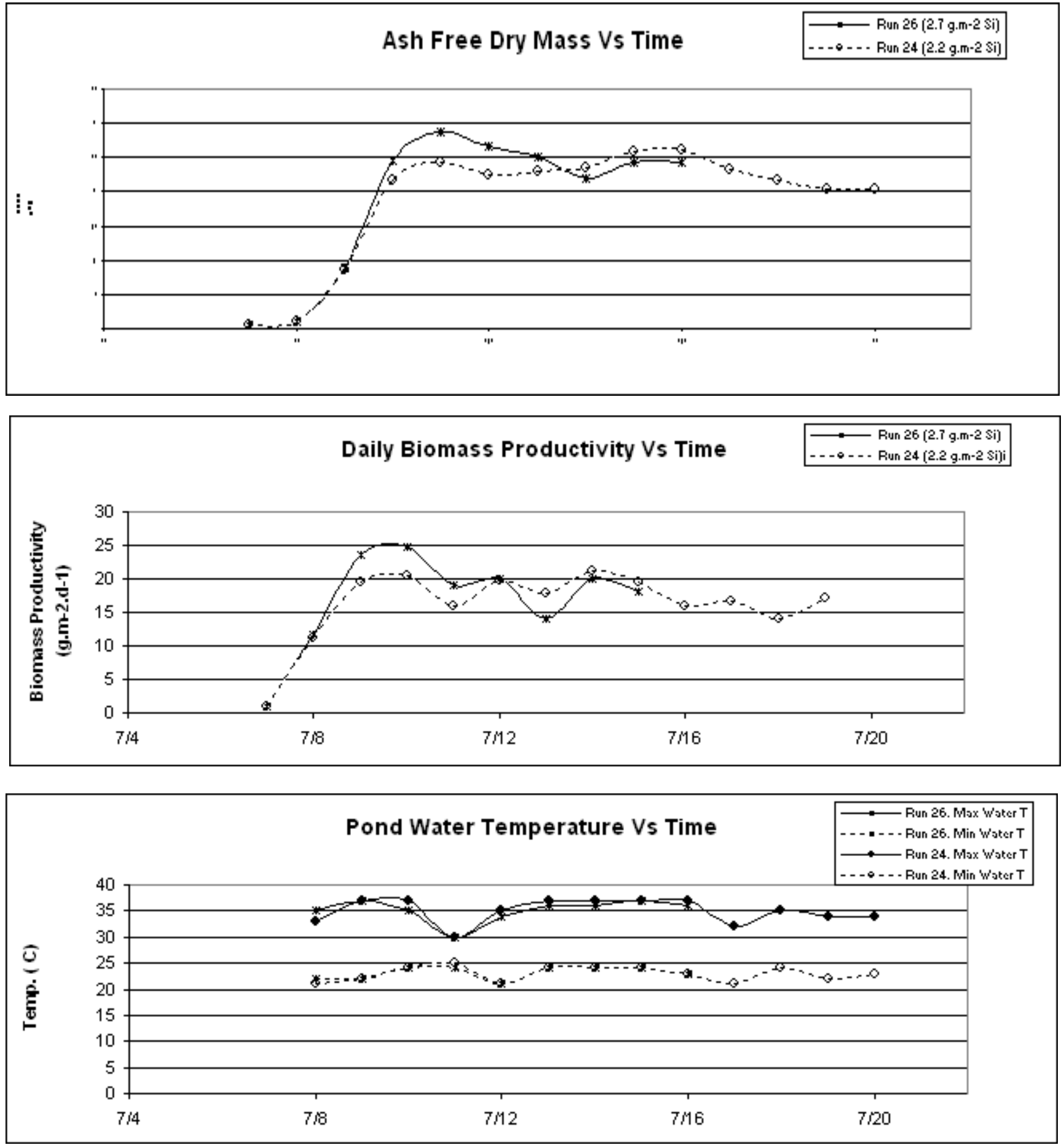
Table 14. Effect of pH on Cyclotella sp. (strain Cyclo J-5) in Semi-continuous Culture in $2.8 \mathrm{~m} 2$ Ponds

\begin{tabular}{|c|c|c|c|c|c|c|c|c|c|}
\hline \multirow[b]{2}{*}{$\mathrm{pH}$} & \multirow{2}{*}{$\begin{array}{c}\text { Start } \\
\text { of } \\
\text { Avg. } \\
\text { Dates }\end{array}$} & \multirow{2}{*}{$\begin{array}{l}\text { End } \\
\text { of } \\
\text { Avg. } \\
\text { Dates }\end{array}$} & \multirow{2}{*}{$\begin{array}{c}\text { Avg } \\
\text { AFDM } \\
\text { (mg.L- } \\
1)\end{array}$} & \multirow[b]{2}{*}{ StDev } & \multirow{2}{*}{$\begin{array}{c}\text { Max } \\
\text { Biomass } \\
\text { Prod. } \\
\text { (g.m-2.d- } \\
\text { 1) }\end{array}$} & \multirow{2}{*}{$\begin{array}{c}\text { Avg } \\
\text { Biomass } \\
\text { Prod. } \\
(\text { g.m-2.d- } \\
\text { 1) }\end{array}$} & \multicolumn{3}{|c|}{$\begin{array}{l}\text { Avg } \\
\text { Photo. } \\
\text { Efficiency }\end{array}$} \\
\hline & & & & & & & StDev & $(\%)$ & StDev \\
\hline 7.5 & $7 / 20$ & $7 / 25$ & 248 & \pm 52.3 & 22.2 & 20.7 & \pm 1.6 & 4.3 & \pm 0.5 \\
\hline 8 & $7 / 20$ & $7 / 25$ & 231 & \pm 43.3 & 23.9 & 19.5 & \pm 3.6 & 4.0 & \pm 0.8 \\
\hline
\end{tabular}

Ave. insolation 6948 W.hr.m-2.d-1

Ave. max temp $37 \mathrm{C}$

Ave. $\min$ temp $26 \mathrm{C}$ 


\subsection{Vertical Flat Plate Photobioreactors vs. Open Raceway Ponds}

Experiments were performed to compare the productivity obtained with the vertical flat plate (VFP) reactors and small raceway (RW) ponds. Initially, Tetraselmis was used as the test organism and then Cyclotella was used. Figure 1b shows the VFP used in these experiments.

During the fall and winter, the insolation falling on the surface of a south facing vertical reactor is higher than that incident on the surface of a horizontal reactor. Thus it was expected that the biomass production (in g.m-2 of reactor surface area) from the VFP reactor would be higher than that from the RW pond, and, conversely, that the photosynthetic efficiency (PE) would be lower. As shown by data taken during November and December (Table 15, also Figure 4a), biomass productivity in the VFP was indeed higher that in the RW ponds by about $50 \%$. For most of this time vertical insolation was not yet being measured. However, at the end of December it was and the data shows that when insolation was about $60 \%$ higher, the (PE) was 10\% lower. This result, that the PE of the horizontal reactor was higher due to the lower incident light intensity, was anticipated to be more pronounced than was actually observed. One explanation is that the temperature during in this period was much lower in the RW ponds compared to the VFP. In December, average minimum pond temperature in the RW open pond averaged only $12 \mathrm{oC}$ compared to $17 \mathrm{oC}$ in the VFP reactor. Significant differences in minimum temperature continued in January and February. There was little difference in the average productivity in the RW pond, or in the VFP reactor, over this whole period of time (Table 15 Figure $4 \mathrm{~b}, \mathrm{c}, \mathrm{d}$ ). This would suggest that temperature, not light, limited biomass production. In March, horizontal insolation became higher than vertical insolation. At this time the RW pond was covered with transparent plastic, resulting in similar reactor temperatures. Now productivities were similar for the two systems, but PE was higher in the VFP as incident light intensity was lower.

In these experiments comparing the two reactor types, the hypothesis that as insolation increases PE decreases, regardless of reactor type, was consistent only with the data taken in late December and March. A major difficulty in achieving the highest productivity allowed by prevailing light levels in outdoor systems is in maintaining optimal temperature. A main advantage of the VFP reactor, or any other moderately shallow reactor without much open water surface, is that temperature is more easily controlled using a groundwater spray, allowing some heating in winter and effective cooling in summer. Of course, such temperature control would be very costly in large-scale systems.

Scatter plots of the daily biomass production were made as functions of insolation, minimum temperature, and maximum temperature to search for correlations among these parameters. The plots (not shown) indicated that there was little linear correlation between any variables using the data from either reactor type. The highest correlation coefficient obtained was for productivity of the VFP reactor against insolation $\left(\mathrm{r}^{2}=0.46\right)$. All of the other correlation coefficients were between 0.05 and 0.18 . However, more visually informative scatter plots of productivity versus insolation, with a log relationship plotted on them, are shown in Figure 5a,b. It is evident that there was a lot less scatter in the VFP results than with the open RW ponds. Again, this is attributed to the capability of controlling temperature within more optimal ranges for the VFP reactors. 
As spring advanced and sun angle increased, the productivity from the East-West oriented VFP reactor became very low, about 6 - $10 \mathrm{~g} / \mathrm{m} 2 / \mathrm{d}$. Table 16a shows several replicate VFP reactor runs using Tetraselmis. Photosynthetic efficiency was 4 - 6\% (always referenced to the photosynthetically active radiation, PAR). The incident light intensity was very low (total daily light of $2500 \mathrm{w} / \mathrm{m} 2$ for the vertical reactor, vs. $7500 \mathrm{w} / \mathrm{m} 2$ for the horizontal ponds). By changing the orientation of the VFP to North-South, the East side would receive more direct light in the mornings, while the West side would receive direct light in the afternoon. Doing this increased the daily insolation to more than $8000 \mathrm{w} / \mathrm{m} 2$. Productivity increased by more than a factor of two to $12-17 \mathrm{~g} / \mathrm{m} 2 / \mathrm{d}$, but PE decreased to 3\% PAR. It was decided that the potential for attaining higher biomass productivity made this orientation more suitable for summer time studies.

It would have been advantageous at this time to continue comparing the growth of Tetraselmis in the two reactor types with the VFP reactor oriented N-S. However, as ambient temperature and humidity increased, it became increasingly difficult to maintain cultures of this alga, as evidenced by disparate results in replicate reactors in June (Table 16a, N-S orientation). The numbers of amoeba and ciliates quickly increased in the cultures, leading to culture crashes. It became impossible to operate either the open RW ponds or the VFP photobioreactor with Tetraselmis. Figure 6 shows several consecutive runs in which the ponds crashed due to protozoa.

The Cyclotella was still growing well in the small ponds, so it became the candidate strain also for the VFP reactor. Replication and reactor comparison experiments were carried out using this alga in the VFP reactors oriented north-south (e.g. facing east-west). The data from the former are shown in Figure 7. The results were very reproducible. In these experiments, the VFP reactor was diluted by $90 \%$ every other day, because it had been observed that both biomass productivity and culture stability were improved with such a dilution schedule.

The data presented in Figure 8 and Table17 show that under similar conditions of insolation and temperature, the VFP and RW pond reactors yielded essentially the same photosynthetic efficiency. The apparent $10 \%$ higher biomass productivity of the VFP is not significant because the area considered for this calculation was only the one-sided area of the reactor. In the North-South orientation used, one side is always facing away from the sun, but still receives scattered light on that side equivalent to $15-20 \%$ of the direct sunlight. All of this light is accounted for in the calculation of PE. Thus, the PE was the same for each of the two types of reactors. In addition, the minimum doubling times (and thus the maximum specific growth rates) of cultures under these conditions were the same, 4.5 hours. This is the most important experiment from the standpoint of comparing the two reactor types. When the conditions, mainly temperature and $\mathrm{pH}$ (e.g. CO2 supply), are controlled to be similar in both of the reactors, the results, in terms of productivity, are similar.

In conclusion, reactor type has no effect on the productivity attainable under similar conditions. 


\section{Table 15}

\section{Small Pond vs Vertical Flat Plate Bioreactor in Semi-continuous Culture \\ (All Tetraselmis Trials)}

Dates of Overall Exp. - (11/7/2003 - 3/31/2004)

\begin{tabular}{|c|c|c|c|c|c|c|c|c|c|c|c|c|}
\hline $\begin{array}{l}\text { Pond } \\
\text { I.D. }\end{array}$ & $\begin{array}{c}\text { Pond } \\
\text { Surface } \\
\text { Area } \\
(\mathrm{m}-2)\end{array}$ & $\begin{array}{l}\text { Algae } \\
\text { Strain }\end{array}$ & $\begin{array}{l}\text { Run } \\
\text { No. }\end{array}$ & $\begin{array}{l}\text { Start of } \\
\text { Avg. } \\
\text { Dates }\end{array}$ & $\begin{array}{c}\text { End of } \\
\text { Avg. } \\
\text { Dates }\end{array}$ & $\begin{array}{c}\text { M ax } \\
\text { AFDM } \\
\text { (mg.L-1) }\end{array}$ & $\begin{array}{c}\text { Avg } \\
\text { AFDM } \\
\text { (m g.L-1) }\end{array}$ & $\begin{array}{c}\text { Max } \\
\text { Biomass } \\
\text { Prod. } \\
\text { (g.m -2.d-1) }\end{array}$ & $\begin{array}{c}\text { Avg } \\
\text { Biomass } \\
\text { Prod. } \\
\text { (g.m-2.d-1) }\end{array}$ & $\begin{array}{c}{ }^{*} \text { Avg } \\
\text { Photo. } \\
\text { Efficiency } \\
(\%)\end{array}$ & $\begin{array}{c}{ }^{*} \text { Avg } \\
\text { Daily } \\
\text { Insulation } \\
(\text { w.hr-1.m-2.d-1) }\end{array}$ & \\
\hline D1 & 2.8 & $\overline{~ T-B C A-1}$ & $\bar{~} 1$ & $11 / 12$ & $11 / 23$ & 188 & $\overline{173}$ & $\bar{~} 13.7$ & 9.6 & * & * & \multirow{2}{*}{ Trial 1} \\
\hline FP1 & 0.6 & T-BCA-1 & 2 & $11 / 12$ & $11 / 23$ & 557 & 489 & 20.4 & 15.3 & * & * & \\
\hline D1 & 2.8 & T-BCA-1 & 3 & $12 / 8$ & $12 / 28$ & 262 & 183 & 16.4 & 9.1 & 4.0 & 3287 & \multirow{2}{*}{ Trial 1} \\
\hline FP1 & 0.6 & T-BCA-1 & 2 & $12 / 8$ & $12 / 28$ & 922 & 566 & 19.8 & 11.4 & * & * & \\
\hline D3 & 2.8 & T-BCA-1 & 5 & $1 / 5$ & $1 / 17$ & 195 & 177 & 12.3 & 8.8 & 3.2 & 3894 & \multirow{2}{*}{ Trial 2} \\
\hline FP1 & 0.6 & T-BCA-1 & 6 & $1 / 5$ & $1 / 17$ & 586 & 522 & 20.2 & 14.1 & 3.3 & 6119 & \\
\hline D3 & 2.8 & T-BCA-1 & 8 & $1 / 25$ & $2 / 11$ & 260 & 197 & 15.1 & 9.2 & 3.5 & 3728 & \multirow{2}{*}{ Trial 3} \\
\hline FP1 & 0.6 & T-BCA-1 & 9 & $1 / 25$ & 2/11 & 743 & 554 & 22.1 & 13.6 & 4.2 & 4629 & \\
\hline D1 & 2.8 & T-BCA-1 & 10 & $3 / 10$ & $3 / 30$ & 267 & 238 & 16.1 & 12.3 & 3.1 & 5699 & \multirow{2}{*}{ Trial 4} \\
\hline FP1 & 0.6 & T-BCA-1 & 11 & $3 / 10$ & $3 / 30$ & 522 & 423 & 17.9 & 11.6 & 4.0 & 4140 & \\
\hline
\end{tabular}


Figure 4a.

Experiment 8, Trial 1. Small Pond vs Vertical Flat Plate Bioreactor Tetraselmis sp. (strain, T-BCA-1) in Small Ponds (2.8m-2) \& Flat Plate Bioreactor (0.6m-2) Semi-continuous Culture.
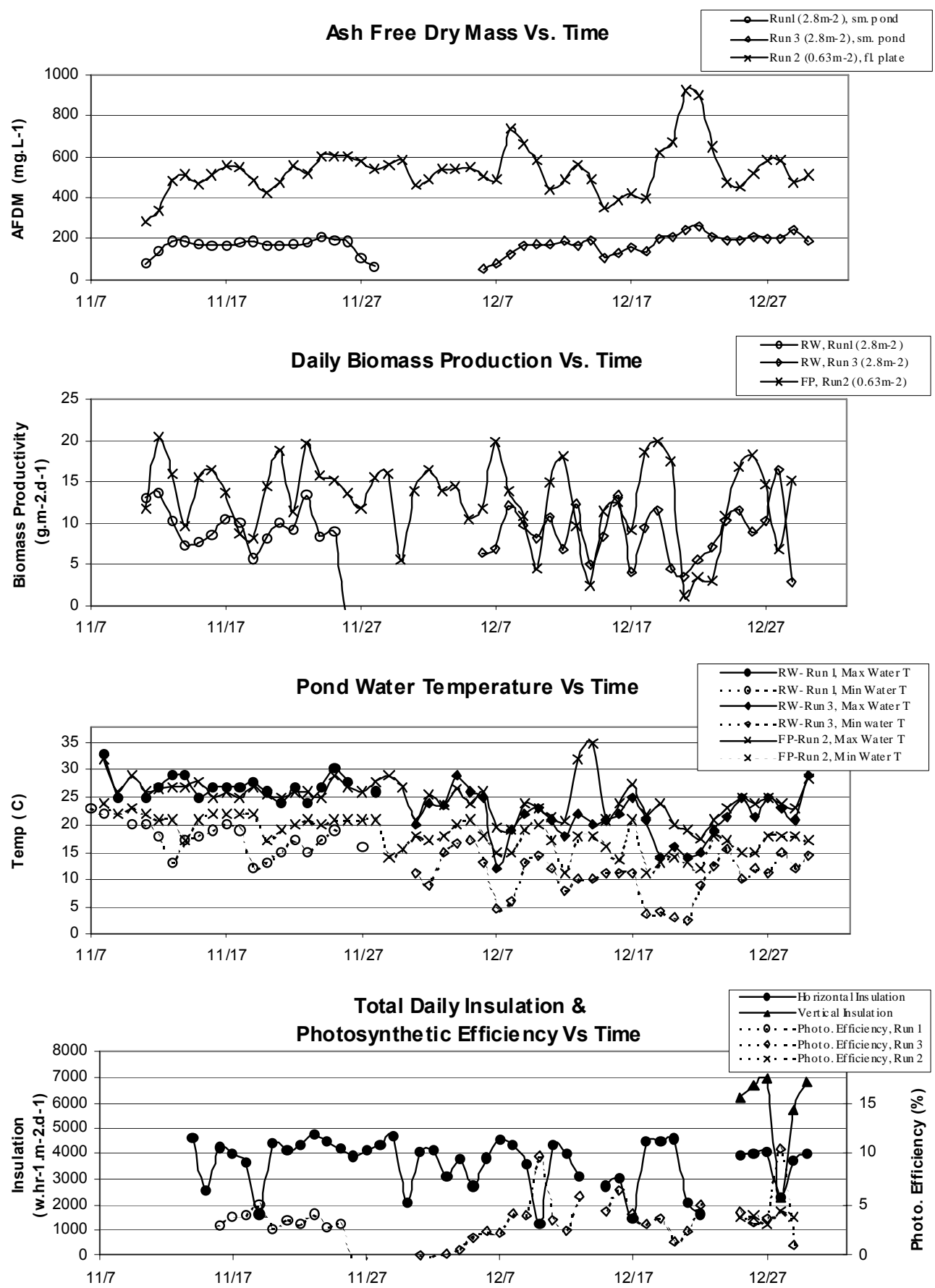
Figure 4b.

Experiment 8, Trial 2. Small Pond vs Vertical Flat Plate Bioreactor Tetraselmis sp. (strain, T-BCA-1) in Small Ponds ( 2.8m-2) \& Flat Plate Bioreactor (0.6m-2) Semi-continuous Culture.

Ash Free Dry Mass Vs Time -R R W Run $5(2.8 \mathrm{~m}-2)$. Unheat ed
$\longrightarrow$-FP, Run $6(0.63 \mathrm{~m}-2)$. Temp Regulated

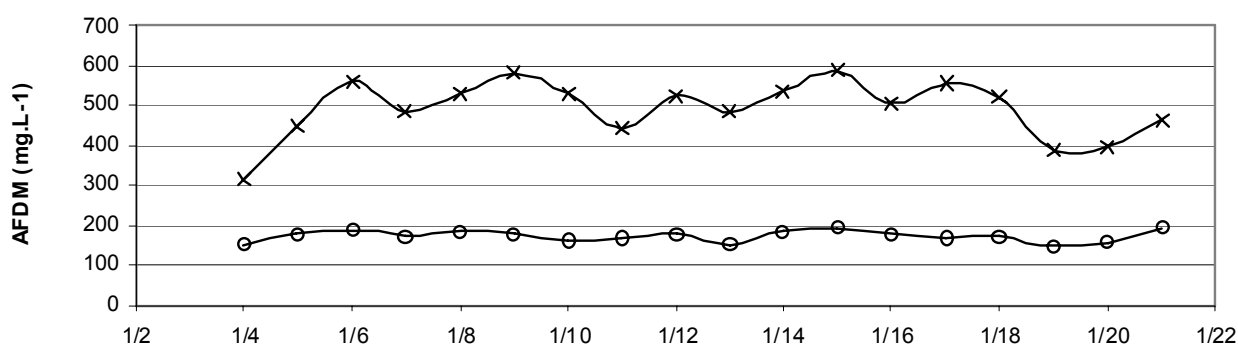

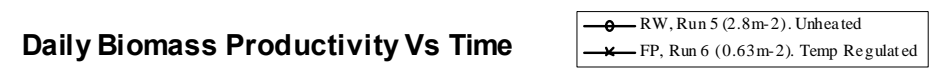
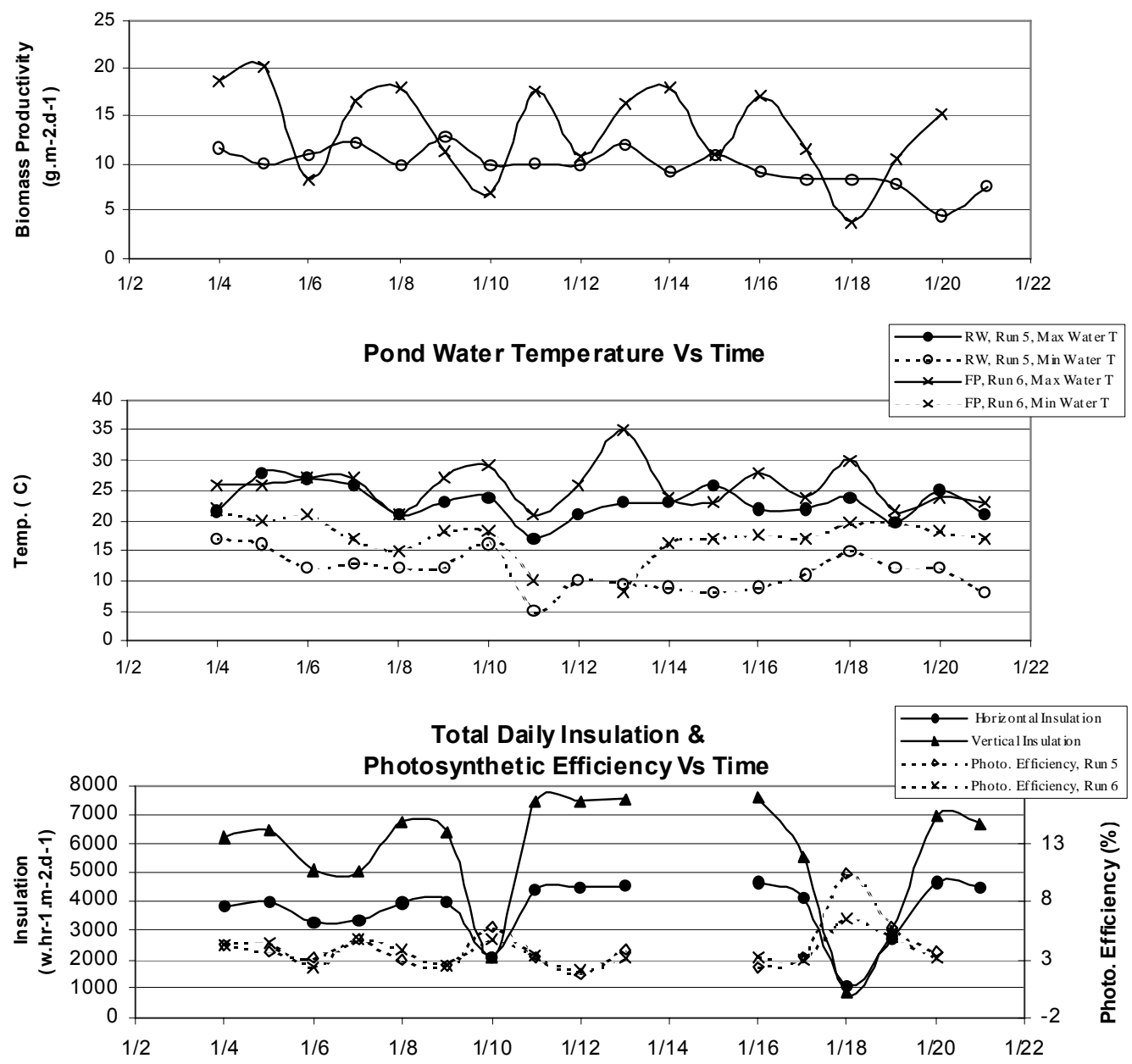
Figure 4c.

Experiment 8, Trial 3. Small Pond vs Vertical Flat Plate Bioreactor Tetra selmis sp. (strain, T-BCA-1) in Small Ponds ( 2.8m-2) \& Flat Plate Bioreactor (0.6m-2) Semi-continuous Culture.
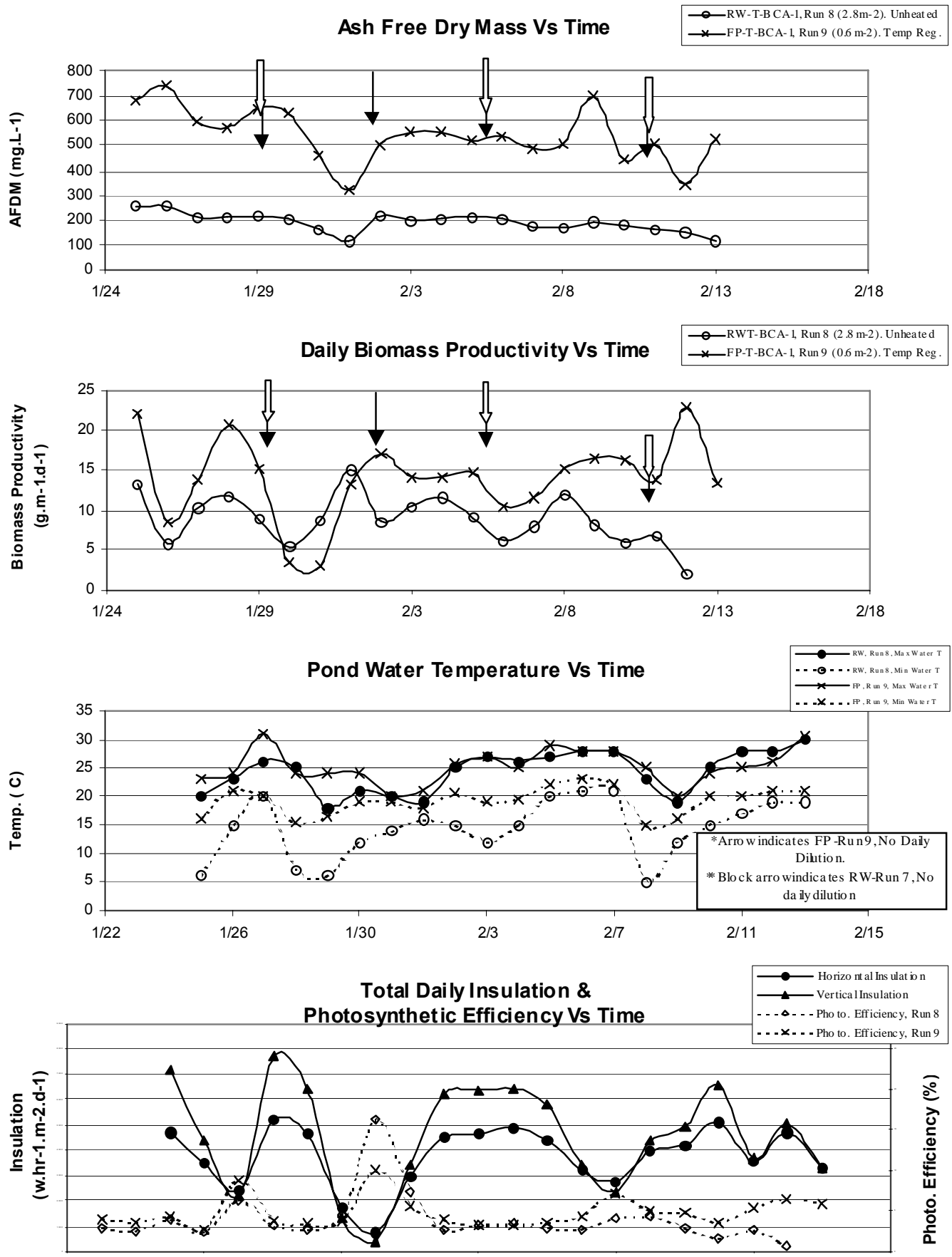
Figure 4d.

Experiment 8, Trial 4. Small Pond vs Vertical Flat Plate Bioreactor Tetraselmis sp. (strain, T-BCA-1) in Small Ponds ( 2.8m-2) \& Flat Plate Bioreactor (0.6m-2) Semi-continuous Culture.
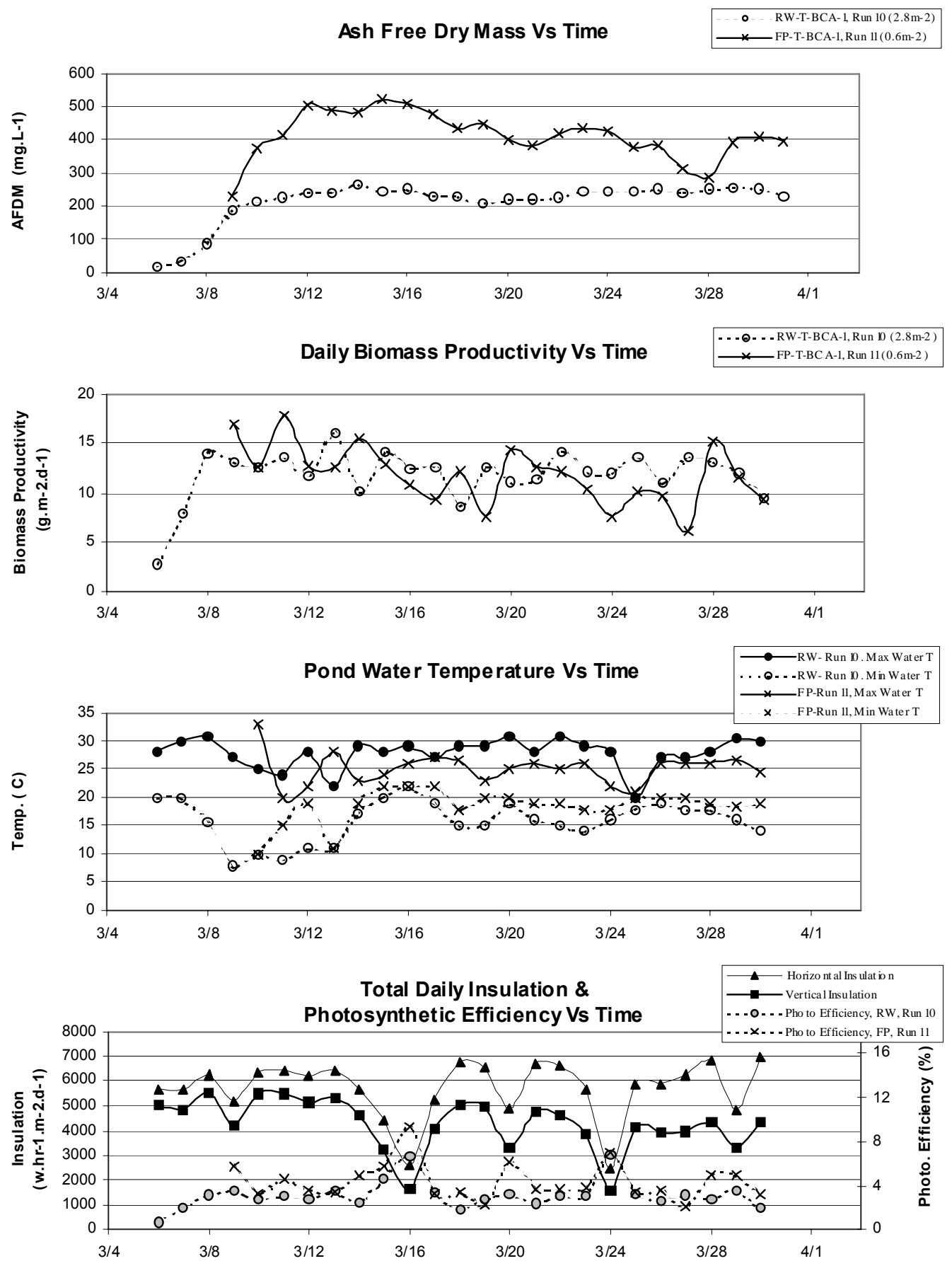
Figure 5a. Tetraselmis. Scatter Plots of Productivity and PE vs Insolation for VFP and RW Pond
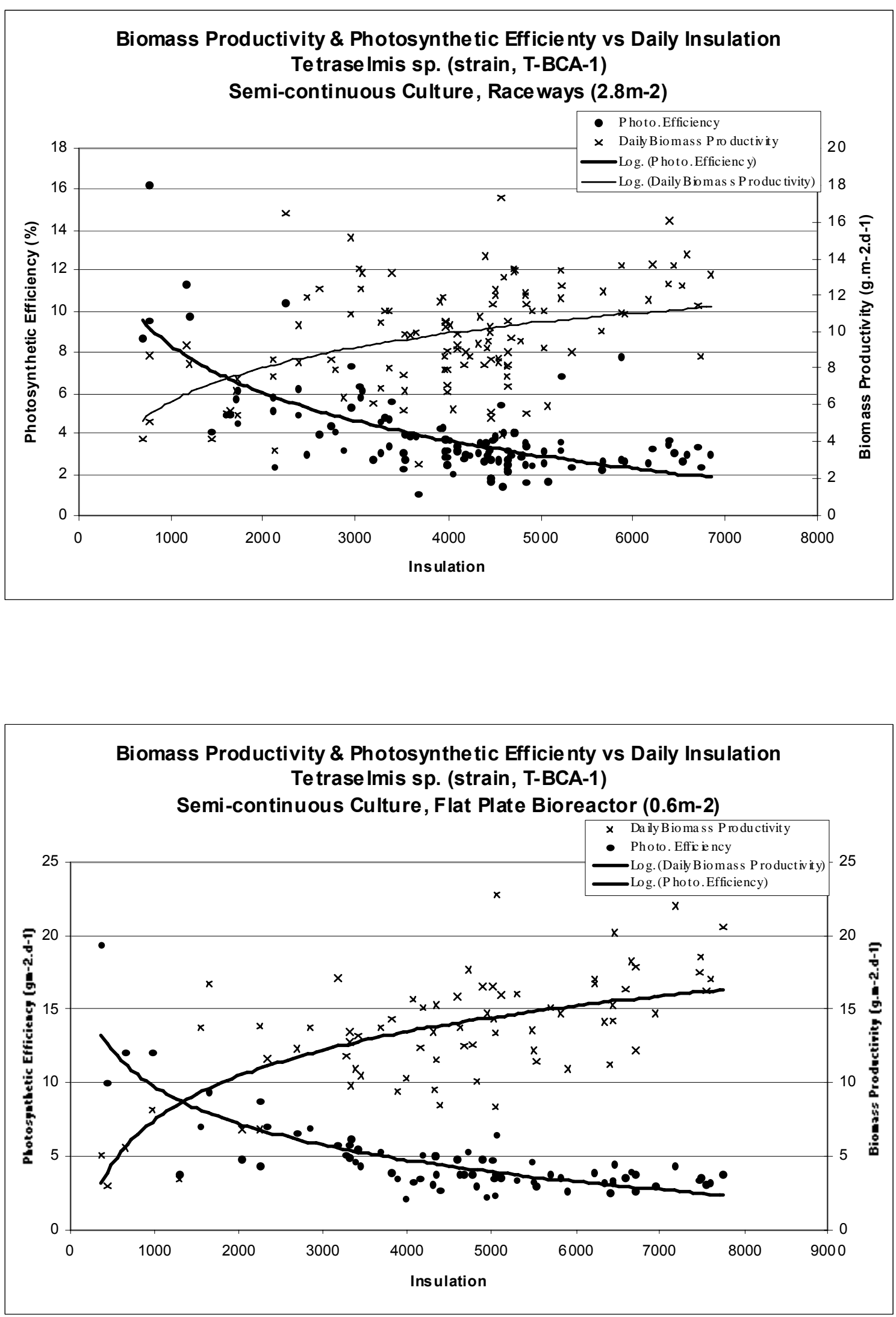
Figure 5b. Cyclotella. Scatter Plots of Productivity and PE vs Insolation for RW Pond

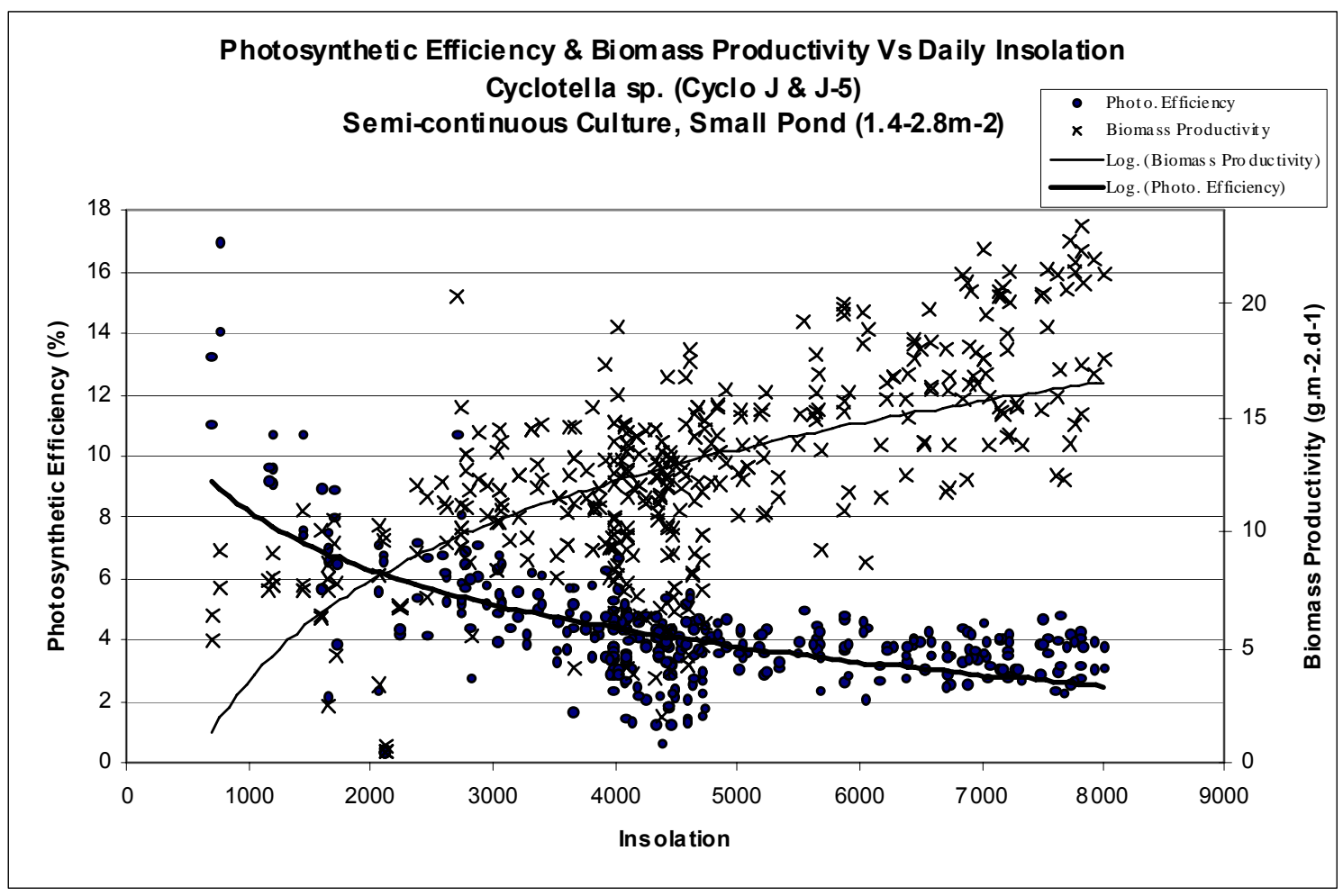


Table 16a. Tetraselmis Replicate Flat Plate Bioreactors FP1 vs FP2.

Strain T-BCA-1 in Semi-continuous Culture.

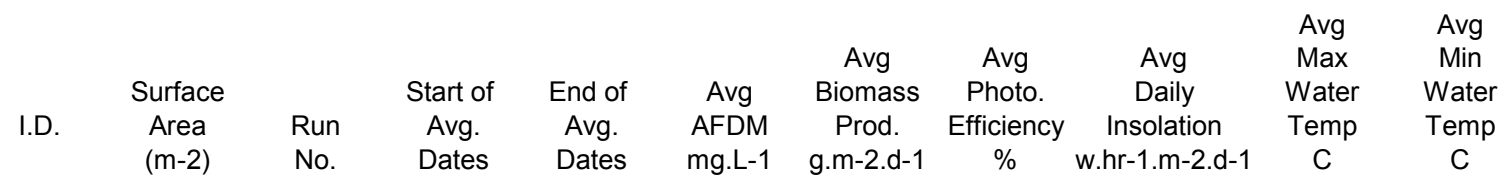

East/West Orientation.

$\begin{array}{lllllllllll}\text { FP1 } & 0.63 & 12 & 4 / 23 & 4 / 27 & 359 & 8.1 & 4.2 & 2775 & 26 & 21 \\ \text { FP2 } & 0.66 & 13 & 4 / 23 & 4 / 27 & 409 & 8.8 & 4.5 & 2775 & 27 & 21 \\ & & & & & & & & & & \\ \text { FP1 } & 0.63 & 14 & 5 / 6 & 5 / 11 & 317 & 10.5 & 5.9 & 2589 & 27 & 21 \\ \text { FP2 } & 0.66 & 13 & 5 / 6 & 5 / 11 & 340 & 9.4 & 5.3 & 2589 & 27 & 20 \\ & & & & & & & & & & \\ \text { FP1 } & 0.6 & 15 & 5 / 14 & 5 / 22 & 258 & 6.5 & 4.0 & 2350 & 29 & 22 \\ \text { FP2 } & 0.6 & 16 & 5 / 14 & 5 / 22 & 302 & 6.6 & 4.0 & 2350 & 29 & 22\end{array}$

North/South Orientation

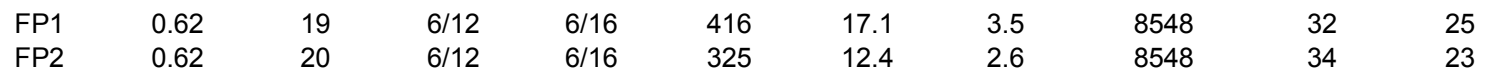

\section{Table 16b. Cyclotella Replicate Flat Plate Bioreactors FP1 vs FP2}

North/South Orientation

Strain Cyclo $\mathrm{J}$ in Semi-continuous culture. Alternating 90\% and 0\% Daily Dilutions

\begin{tabular}{|c|c|c|c|c|c|c|c|c|}
\hline I.D. & $\begin{array}{c}\text { Surface } \\
\text { Area } \\
(\mathrm{m}-2) \\
\end{array}$ & $\begin{array}{l}\text { Run } \\
\text { No. }\end{array}$ & $\begin{array}{c}\text { Start of } \\
\text { Avg. } \\
\text { Dates } \\
\end{array}$ & $\begin{array}{c}\text { End of } \\
\text { Avg. } \\
\text { Dates }\end{array}$ & $\begin{array}{c}\text { Avg } \\
\text { AFDM } \\
\text { mg.L-1 } \\
\end{array}$ & $\begin{array}{c}\text { Max } \\
\text { Biomass } \\
\text { Prod. } \\
\text { g.m-2.d-1 }\end{array}$ & $\begin{array}{c}\text { Avg } \\
\text { Biomass } \\
\text { Prod. } \\
\text { g.m-2.d-1 }\end{array}$ & $\begin{array}{c}\text { Avg } \\
\text { Photo. } \\
\text { Efficiency } \\
\% \\
\end{array}$ \\
\hline $\mathrm{FF}$ & 0.67 & 27 & $7 / 30$ & $8 / 11$ & 451 & 26.0 & 18.1 & 5.6 \\
\hline FP2 & 0.67 & 33 & $7 / 30$ & $8 / 11$ & 446 & 27.3 & 18.9 & 5.8 \\
\hline
\end{tabular}

Avg. max. water temp: $32 \mathrm{C}$

Avg. Min. water temp.: $24 \mathrm{C}$

Avg daily insolation: 4626 w.hr-1.m-2.d-1 


\section{Figure 6}

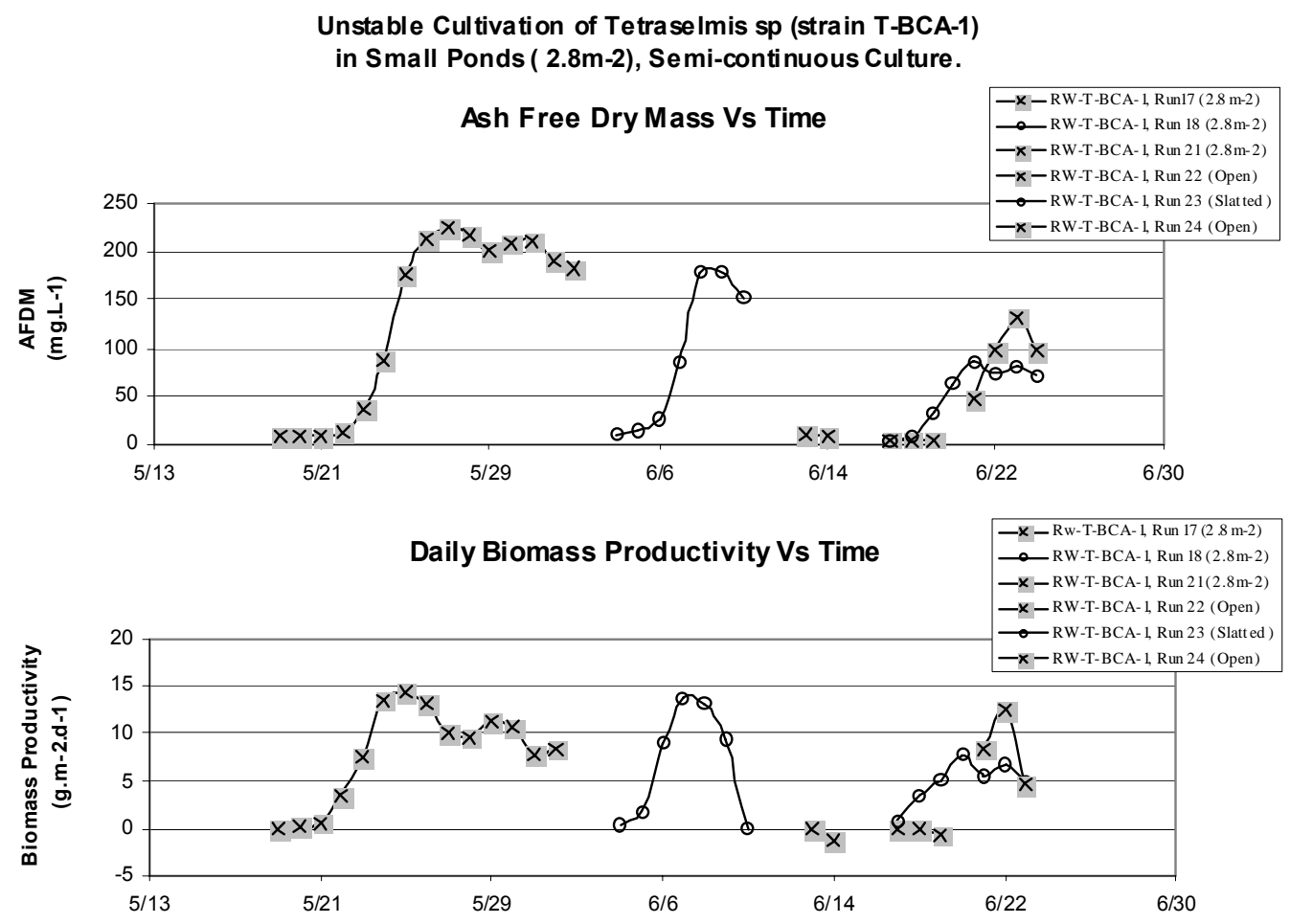

Pond Water Temperature Vs Time

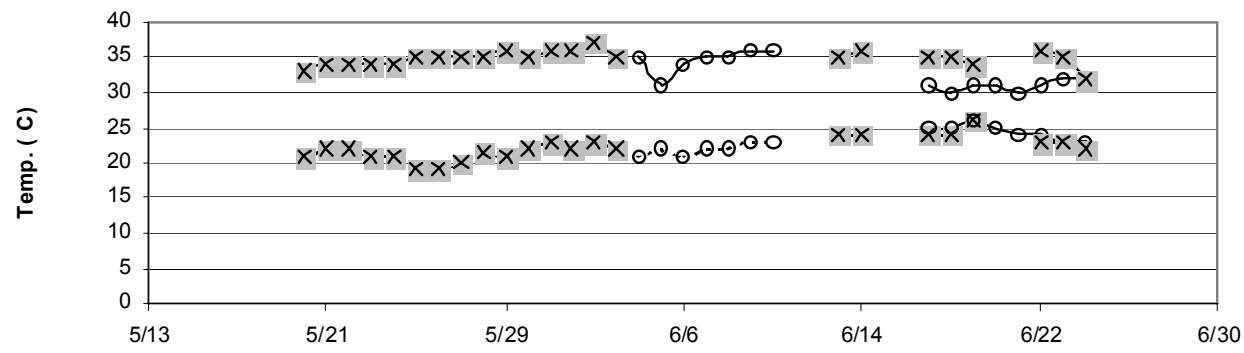

Total Daily Insolation \&

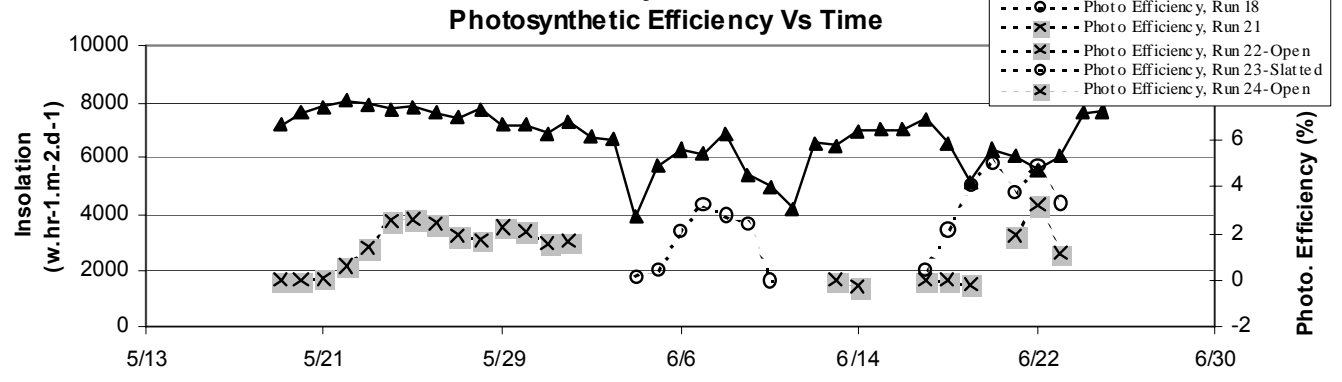


Figure 7.

Experiment 12, Trial 2. Flat Plate Bioreactors FP1 vs FP2. Cyclotella sp. (Cyclo J) in Semi-continuous Culture. Al ternating $\mathbf{9 0 \%}$ and $0 \%$ Daily Dilutions

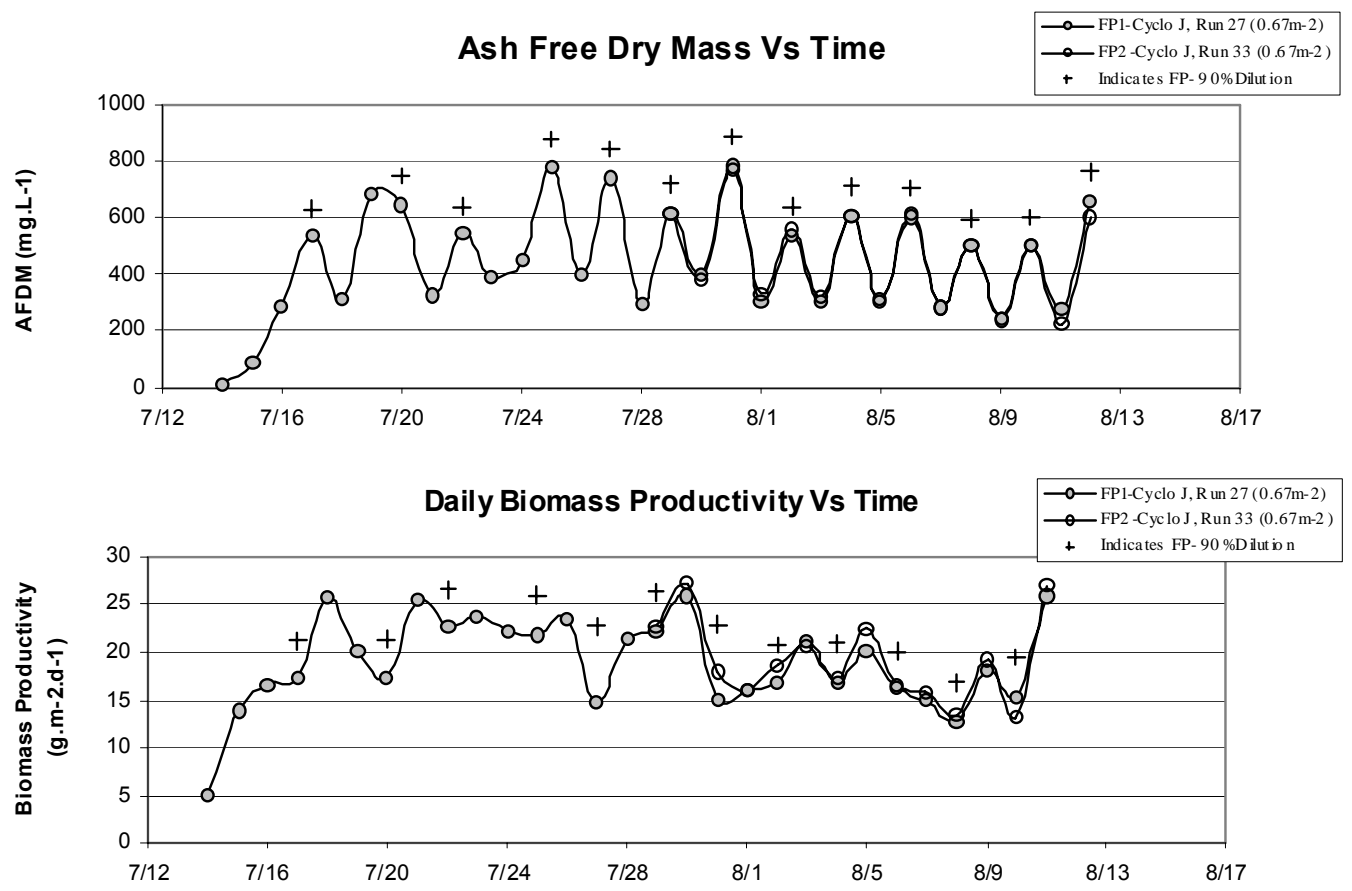

Pond Water Temperature Vs Time

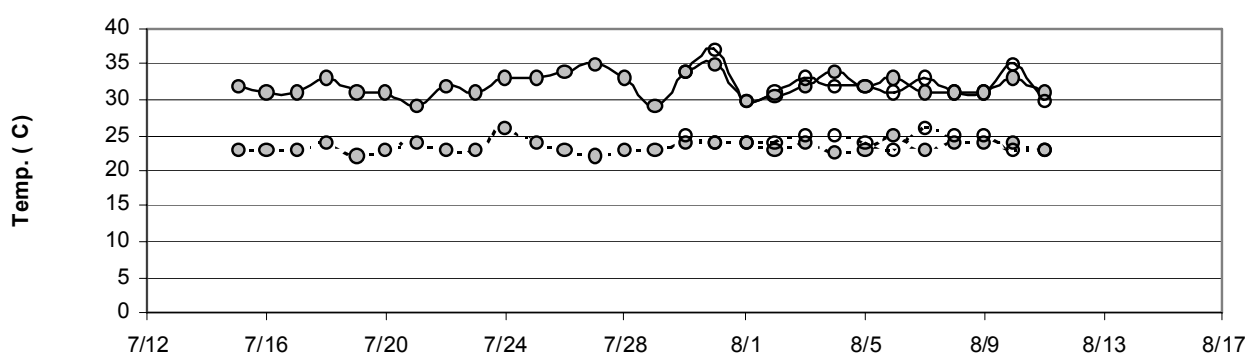

Total Daily Insolation \&

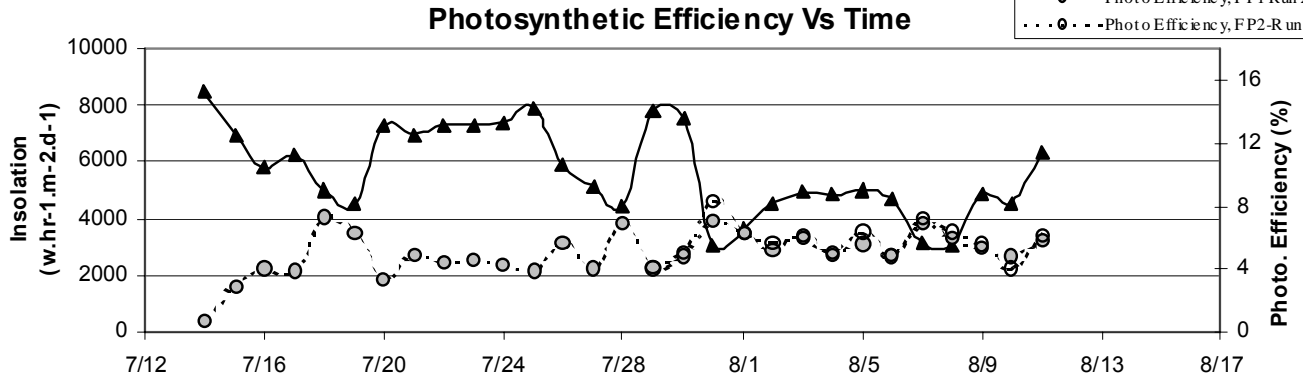




\section{Figure 8.}

Flat Plate Bioreactor, $(0.67 \mathrm{~m}-2)$ vs Small Pond $(2.8 \mathrm{~m}-2)$

Cyclotella sp. (Cyclo J \& J-5) in Semi-continuous Culture.
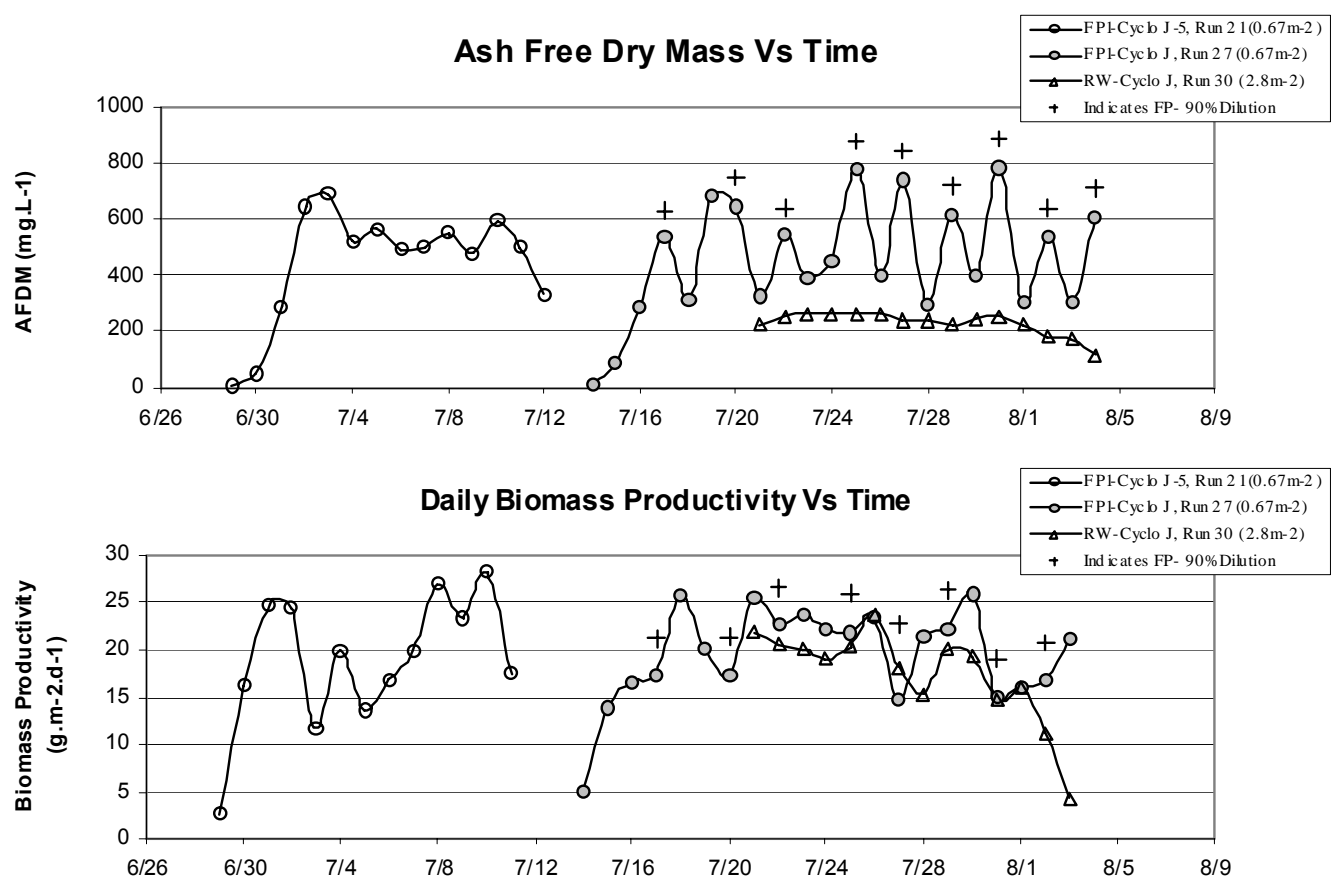

Pond Water Temperature Vs Time

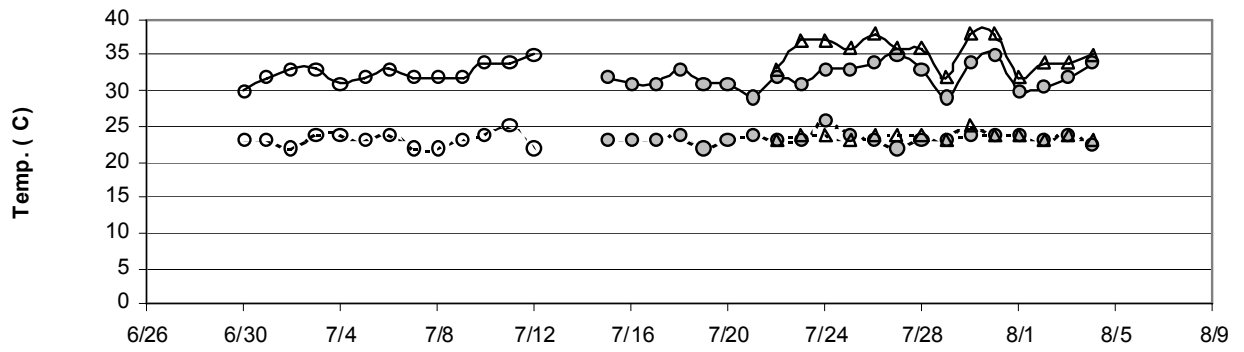

Total Daily Insolation \&

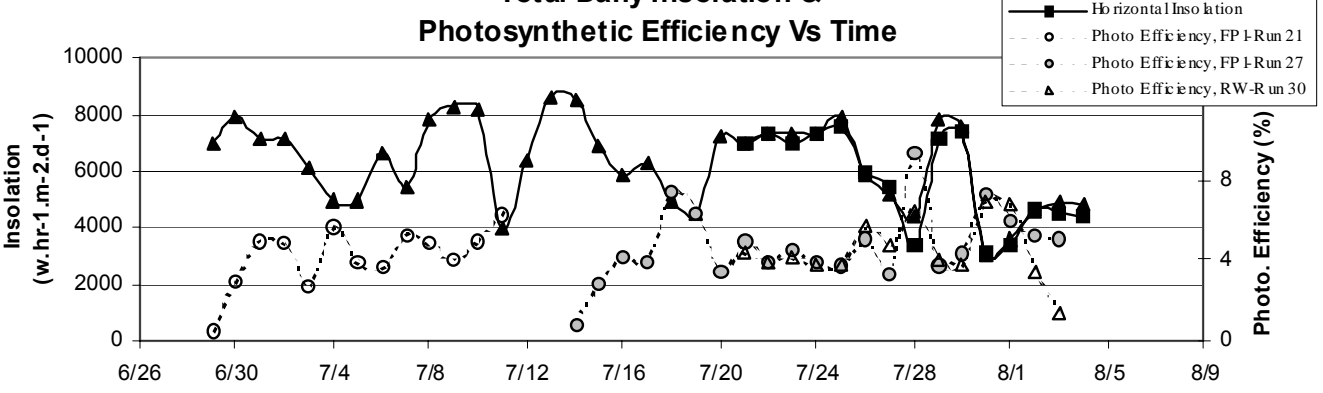




\section{Table 17.}

Small Pond vs Flat Plate for Cyclotella sp. J in Semi-continuous culture

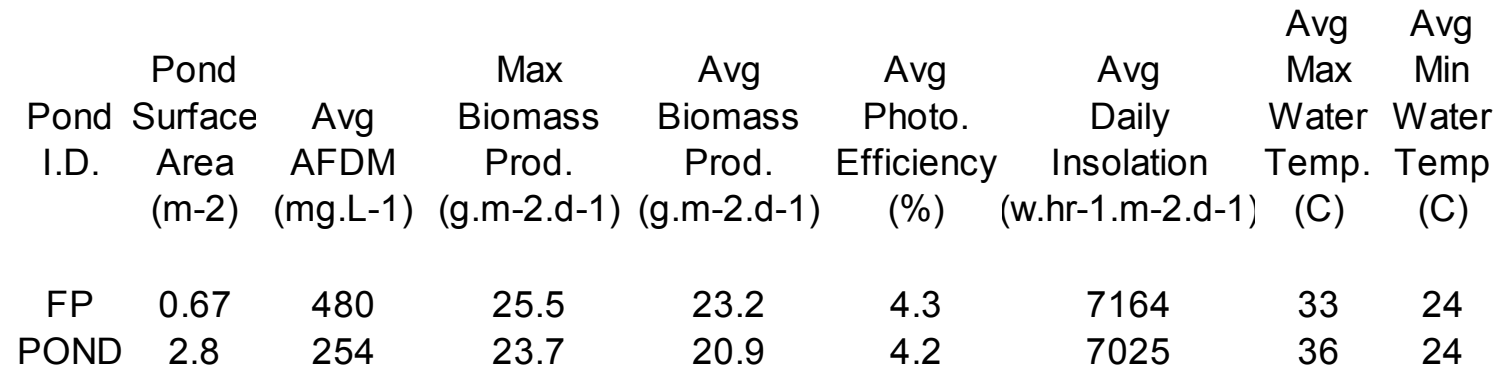

Averages from $7 / 21-7 / 26 / 2004$, when insolation was nearly contant. 


\subsection{Experiments with Photosynthetic Mutants}

Photosynthesis mutant strains were produced by Prof. Juergen Polle at Brooklyn College (see the Appendix for his detailed report). The strains were pre-screened to establish that pigment content was indeed decreased compared to the wild type. In some of these cases, photosynthetic parameters were measured to determine whether they were altered in ways presumed to be more favorable for biomass production at high insolation.

The original Cyclotella sp wild type (CYCLOJ) was the result of an enrichment process conducted over ten years ago at the Sea-Ag, Inc., facility. It has been maintained since then in the outdoor ponds under the enrichment condition. Periodically, samples were plated and individual colonies (clones) were picked to grow test tube and flask cultures in the laboratory. Many of these have been maintained in the lab, offering sources of unialgal inoculum for study. It is from one of these clones, CYCLOJ5, that samples were grown to provide wild type strains for mutagenesis. The outdoor wild type and the clones isolated from it appear identical, but are not. As will be discussed below, maximum specific growth rates are a little higher for the CYCLOJ. It is also much more easily propagated outdoors, and less easily so indoors than clone CYCLOJ5.

Five mutant strains of Cyclotella provided by Prof. Polle were grown in our laboratory to produce the inoculum for the outdoor ponds. Pond cultures of the wild type strains were operated in parallel to the cultures of mutants. Pictures of four of the mutant cultures are shown in Figure 9. The degree of depigmentation is evident from the photos. CM7 is almost as coffee brown as the wild type. CM3 is orange-tan while CM1 is olive green. The mutant strain CM1-1 was derived from CM1 by further mutagenesis. As a result it lost most of the brown fucoxanthin pigment, so it appears green. Results from Dr. Polle lab demonstrated that this strain contained less chlorophyll as well.

The results are summarized in Table 18. The difference between the productivity obtained from the wild type and mutants M1, M3, and M7 were quite small. M2 and M1-1 were much less productive than the wild type. Thus, at best, the mutants were no better than the wild type at using light incident at high intensity despite lower pigmentation. Measurements of the photosynthetic capacity (oxygen evolution versus light intensity) of the wild types and the mutants were performed at Brooklyn College. Results indicate that only one of the mutants, CM1-1 differed from the wild type in its photosynthetic parameters. It exhibited a higher saturating light intensity, which is what we were looking for. Thus, for the other mutants, it is not surprising that the yields outdoors, where incident irradiance is high, were not better than the wild type. The mutations may have caused not only a decrease in pigmentation in harvesting (antenna) pigments, but also may have reduced the number of photosynthetic reaction centers thus not changing the saturating irradiance. We anticipated that CM1-1 would perform better since it exhibited a higher irradiance for the onset of light saturation of photosynthesis. But this was not the case. It is possible that the mutation process affected other sites which somehow limited net photosynthetic production. Whatever the reason, the consistent result was that none of the mutants was more productive than the wild type. The experimental results for most of the trials are shown in Table 19. 
There are two other measurements which help to determine whether the mutants were generally competent, or deficient in some unknown way. The first is the maximum specific growth rate attained (minimum doubling time) at low cell density, when light is totally saturating the photosynthetic systems. Table 20 shows the data from the cultures of mutants. As discussed in detail in a subsequent section of this report, the minimum doubling for CYCLOJ5 was about 5 hours. It was substantially longer for the mutants: about 30\% longer for M1, about $80 \%$ longer for M1-1, about $50 \%$ for M2, about $40 \%$ for M2 and about $60 \%$ for M7. Further, most of the cultures of mutants exhibited a prolonged lag time after inoculation. This can bias, or even obscure the measurement of growth rate, yielding artificially long doubling times. This is likely to have happened here. Figure 10a shows the lag in the initial rise in biomass concentration for three trials runs of CM1-1. Although the mutations were not disabling cell growth, as evidenced by the biomass productivities achieved, long lag times and slow maximal growth rates indicate that the mutation process affected more than just light harvesting pigment content.

The second measure of culture robustness is the length of time that cultures can be maintained. Wild type cultures were maintained for weeks to months, with CYCLOJ enduring longer than CYCLOJ5. They usually crashed only when blooms of predators or competitors, originating from the wall growth, occurred, or when addition of too much Si caused clumps to form. On the other hand, the mutant cultures could never be maintained in good condition for more than 7 to 10 days. It can be seen from Figure 10a that the biomass concentration of CM1-1 did not exceed $140 \mathrm{mg}$.L-1, which is low. The wild type grows, in batch, up to nearly $1000 \mathrm{mg} . \mathrm{L}-1$. Although from the figure it looks as if only one of the trials ended up with decreasing biomass concentration, the cultures in the other trials either completely collapsed or became contaminated with other diatoms including the wild type, after the last day shown. The cell surfaces became "dirty", the cells began to flocculate, and finally the culture crashed (experienced a fast decline in biomass density) within a few days. It may well be that the reduction in pigment content along with a reduction in light energy processing centers, rendered the mutant more susceptible to photo inhibition. Figure 10b shows the low and unsustainable productivities from these runs of CM!-1.

Tetraselmis mutant M1 was inoculated twice into a RW pond, once at the end of June and in July. It was not possible to maintain this alga, wild type or mutant in outdoor culture at this time, as discussed in the previous section. Due to the instability of cultivation, it was also not possible to obtain enough data to compare the productivity of any of the Tetraselmis cultures. 
Figure 9.

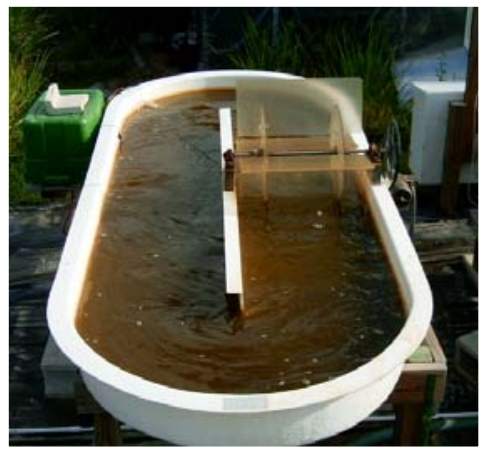

D3

4/27/2004

Cyclotella sp. (Cy-M3),

Run 1

$\mathrm{AFDM}=131$

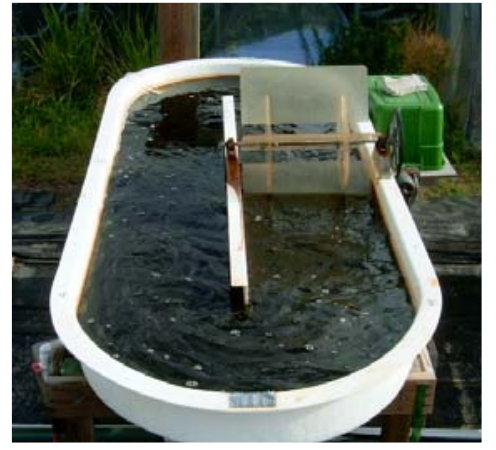

D2

$4 / 27 / 2004$

Cyclotella sp. (Cy-M7),

Run 1

AFDM =

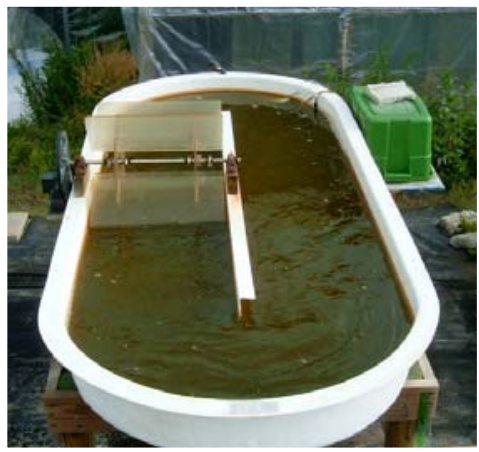

D1

$4 / 27 / 2004$

Cyclotella sp. (Cy-M1),

Run 3

$\mathrm{AFDM}=273$

CM1-1

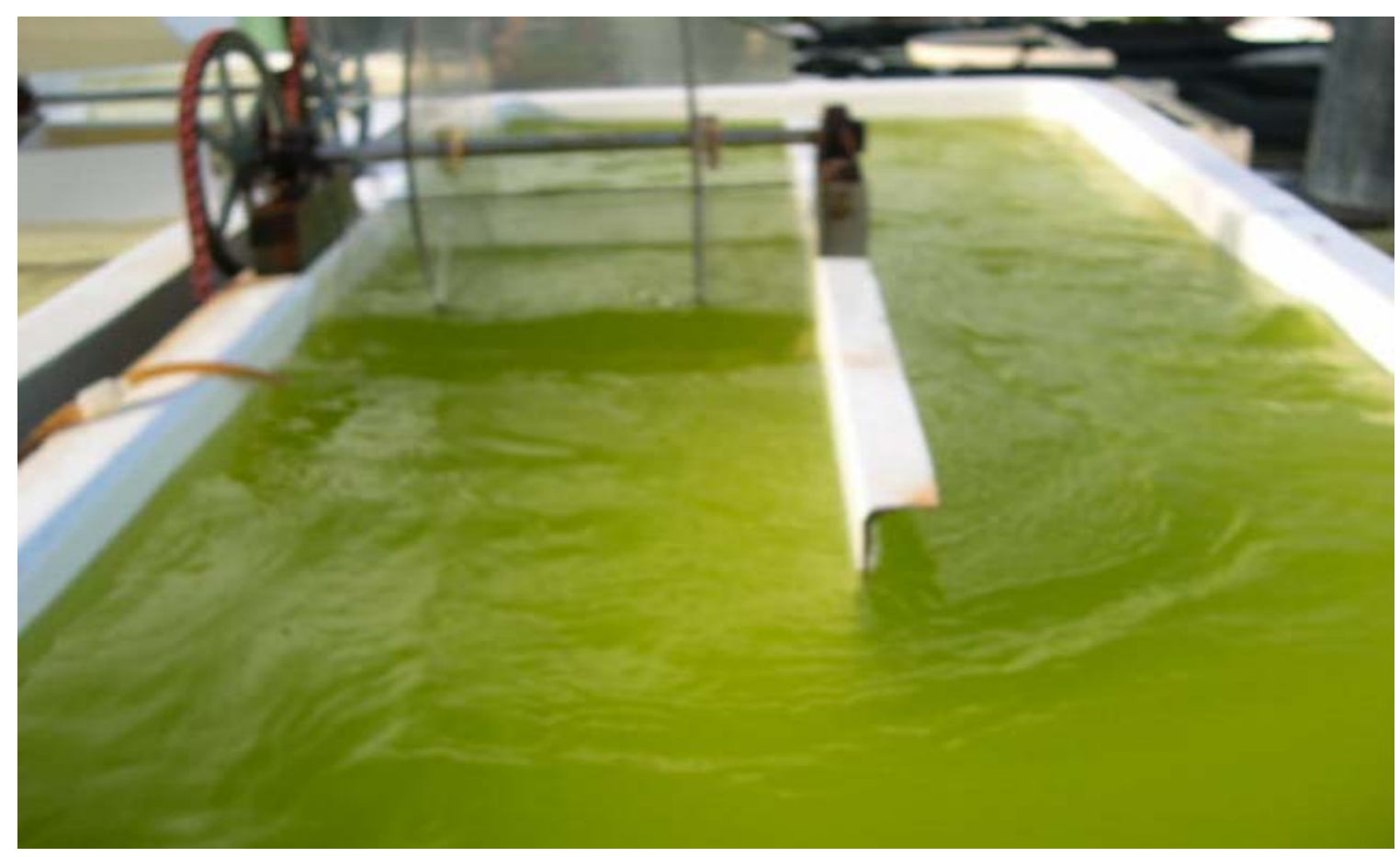




\section{Table 18.}

\section{Summary of Cyclotella Wild Type and Mutant Strains}

(Ratio of Mutant Avg Daily Biomass Production to Wild Type Avg Daily Biomass Production)

- Cyclo M1 - 0.92

- Cyclo M2 - 0.74

- Cyclo M7 - 0.95

- Cyclo M3 - 0.89

- Cyclo M1-1 - 0.6 


\section{Table 19.}

\section{Growth of Cyclotella Mutants in Outdoor Ponds}

\begin{tabular}{|c|c|c|c|c|c|c|c|c|c|c|}
\hline $\begin{array}{l}\text { Algae } \\
\text { Strain } \\
\end{array}$ & $\begin{array}{c}\text { Start of } \\
\text { Avg. } \\
\text { Dates } \\
\end{array}$ & $\begin{array}{c}\text { End of } \\
\text { Avg. } \\
\text { Dates } \\
\end{array}$ & $\begin{array}{c}\text { Max } \\
\text { Biom ass } \\
\text { Prod. } \\
\text { (g.m-2.d-1) } \\
\end{array}$ & $\begin{array}{c}\text { Avg } \\
\text { Biom ass } \\
\text { Prod. } \\
\text { (g.m -2.d-1) } \\
\end{array}$ & $\begin{array}{c}\text { Avg } \\
\text { Photo. } \\
\text { Efficiency } \\
(\%) \\
\end{array}$ & $\begin{array}{c}\text { Avg } \\
\text { Daily } \\
\text { Insolation } \\
\text { (w.hr-1.m-2.d-1) } \\
\end{array}$ & $\begin{array}{c}\text { Min } \\
\text { Doubling } \\
\text { Time } \\
(\mathrm{Hrs}) \\
\end{array}$ & $\begin{array}{c}\text { Avg } \\
\text { Max } \\
\text { Water } \\
\text { Temp } \\
\text { ( c) } \\
\end{array}$ & $\begin{array}{c}\text { Avg } \\
\text { Min } \\
\text { Water } \\
\text { Temp } \\
\text { (C) } \\
\end{array}$ & \\
\hline Cyclo-J & $3 / 7$ & $3 / 27$ & 19.5 & 15.6 & 3.9 & 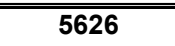 & & 28 & 15 & Wild Strain \\
\hline Cyclo-M1 & $3 / 7$ & $3 / 27$ & 21.1 & 14.5 & 3.7 & 5626 & 7.5 & 27 & 16 & Mutant Strain \\
\hline Cyclo-J & $4 / 14$ & $4 / 22$ & 22.9 & 17.0 & 3.4 & 7033 & & F 28 & F 14 & Wild Strain \\
\hline Cyclo-M 1 & $4 / 14$ & $4 / 22$ & 19.2 & 14.9 & 3.0 & 7033 & 5.4 & $r 28$ & 15 & Mutant Strain \\
\hline Cyclo-J & $5 / 2$ & $5 / 5$ & 20.9 & 15.0 & 4.0 & 5183 & & $r 32$ & $=20$ & Wild Strain \\
\hline Cyclo-M 1 & $5 / 2$ & $5 / 5$ & 16.6 & 14.0 & 3.9 & 5183 & 6.3 & 732 & 21 & Mutant Strain \\
\hline Cyclo-J & $5 / 15$ & $5 / 21$ & 19.0 & 16.2 & 3.1 & 7392 & & F 34 & 21 & Wild Strain \\
\hline Cyclo-M 1 & $5 / 15$ & $5 / 21$ & 19.9 & 16.8 & 3.2 & 7392 & 5.2 & r 33 & 21 & Mutant Strain \\
\hline Cyclo-J & $5 / 29$ & $6 / 3$ & 20.2 & 15.2 & 3.1 & 6992 & & $r 36$ & 22 & Wild Strain \\
\hline Cyclo-M1b & $5 / 29$ & $6 / 3$ & 15.7 & 14.3 & 2.9 & 6992 & 8.1 & 36 & 22 & Mutant Strain \\
\hline Cyclo J & $7 / 21$ & $7 / 24$ & 21.8 & 20.3 & 4.0 & 7146 & & 36 & 24 & Wild Strain \\
\hline Cyclo-M1 & $7 / 21$ & $7 / 24$ & 22.4 & 18.4 & 3.7 & 7146 & 7 & 36 & 23 & Mutant Strain \\
\hline Cyclo-J & $4 / 16$ & $4 / 22$ & 18.8 & 16.3 & 3.3 & 6936 & & 29 & 16 & Wild Strain \\
\hline Cyclo-M2 & $4 / 16$ & $4 / 22$ & 14.7 & 13.8 & 2.8 & 6936 & 6.6 & 28 & 16 & Mutant Stre \\
\hline Cyclo J-5 & $6 / 25$ & $6 / 27$ & 20.6 & 20.5 & 4.0 & 7254 & 6.1 & 38 & 24 & Wild Strain \\
\hline Cyclo-M2 & $6 / 25$ & $6 / 27$ & 14.5 & 12.7 & 2.5 & 7254 & 8.4 & 37 & 24 & Mutant Stre \\
\hline Cyclo-J & $4 / 29$ & $5 / 4$ & 20.3 & 15.9 & 4.6 & 4953 & & 31 & 21 & Wild Strain \\
\hline Cyclo-M3 & $4 / 29$ & $5 / 4$ & 17.2 & 14.2 & 3.8 & 4953 & 6.7 & 31 & 21 & Mutant Stre \\
\hline Cyclo-J & $4 / 27$ & $4 / 29$ & 19.4 & 15.8 & 4.4 & 5090 & & 29 & 19 & Wild Strain \\
\hline Cyclo-M7 & $4 / 27$ & $4 / 29$ & 17.0 & 15.1 & 3.6 & 5090 & 8.6 & 32 & 19 & Mutant Stre \\
\hline Cyclo-J & $5 / 15$ & $5 / 20$ & 19.0 & 16.3 & 3.2 & 7321 & & 34 & 21 & Wild Strain \\
\hline Cyclo-M7 & $5 / 15$ & $5 / 20$ & 17.0 & 15.1 & 2.8 & 7321 & 7.9 & 33 & 22 & Mutant Stre \\
\hline
\end{tabular}


Table 20.

Specific Growth Data for Cyclotella sp Mutant Strains

\begin{tabular}{|c|c|c|c|c|c|c|c|c|c|}
\hline $\begin{array}{c}\text { Algae } \\
\text { Type }\end{array}$ & $\begin{array}{c}\text { Run } \\
\# \\
\end{array}$ & Date & $\begin{array}{c}\text { Daylight } \\
\text { (Hrs) }\end{array}$ & $\begin{array}{c}\text { Sample } \\
\text { Interval } \\
\text { (Hrs) } \\
\end{array}$ & $\begin{array}{c}\text { Culture } \\
\text { Age of } \\
\text { lowest } \\
\text { Doubling } \\
\text { Time (Day) } \\
\end{array}$ & $\begin{array}{c}\text { AFDM E } \\
\text { Start } \\
\text { (mg.L-1) } \\
\end{array}$ & $\begin{array}{c}\text { ndpoints } \\
\text { Finish } \\
\text { (mg.L-1) } \\
\end{array}$ & $\begin{array}{c}\text { Doubling } \\
\text { Time } \\
\text { (Hrs) } \\
\end{array}$ & $\begin{array}{c}\text { Open } \\
\text { Area } \\
(\%) \\
\end{array}$ \\
\hline Cyclo M1 & 1 & $3 / 3$ & 11.7 & 11.7 & 2 & 22.4 & 71.4 & 7.0 & 100 \\
\hline Cyclo M1 & 3 & $4 / 10$ & 11.9 & 11.9 & 1 & 13.5 & 60.7 & 5.4 & 100 \\
\hline Cyclo M1 & 4 & $4 / 24$ & 12.2 & 12.8 & 1 & 8.0 & 30.6 & 6.6 & 100 \\
\hline Cyclo M1 & 5 & $5 / 7$ & 12.6 & 12.1 & 2 & 36.0 & 101.4 & 8.1 & 100 \\
\hline Cyclo M1 & 6 & $5 / 13$ & 12.8 & 12.8 & 1 & 11.3 & 46.4 & 6.2 & 100 \\
\hline Cyclo M1b & 1 & $5 / 27$ & 13.7 & 13.7 & 1 & 12.2 & 48.1 & 6.9 & 100 \\
\hline \multirow[t]{2}{*}{ Cyclo M1 } & 7 & $7 / 18$ & 13.7 & 14.3 & 1 & 13.4 & 68.0 & 6.1 & 100 \\
\hline & & & & & & & $\begin{array}{r}\text { Avg. } \\
\text { StDev. }\end{array}$ & $\underline{\underline{6.6}}$ & \\
\hline Cyclo M1-1 & 2 & $7 / 22$ & 12.7 & 12.7 & 1 & 19.0 & 42.7 & 10.3 & 100 \\
\hline \multirow[t]{2}{*}{ Cyclo M1-1 } & 3 & $7 / 29$ & 12.7 & 11 & 0 & 4.3 & 10.9 & 8.2 & 100 \\
\hline & & & & & & & $\begin{array}{r}\text { Avg. } \\
\text { StDev. }\end{array}$ & $\begin{array}{l}9.3 \\
1.5 \\
\end{array}$ & \\
\hline Cyclo M2 & 1 & $4 / 10$ & 11.9 & 11.9 & 1 & 7.5 & 25.9 & 6.6 & 100 \\
\hline \multirow[t]{2}{*}{ Cyclo M2 } & 3 & $6 / 22$ & 13.9 & 13.9 & 2 & 19.5 & 62.1 & 8.3 & 100 \\
\hline & & & & & & & $\begin{array}{r}\text { Avg. } \\
\text { StDev. }\end{array}$ & $\underline{7.4}$ & \\
\hline Cyclo M3 & 1 & $4 / 25$ & 12.3 & 12.3 & 2 & 29.2 & 97.3 & 7.0 & 100 \\
\hline \multirow[t]{2}{*}{ Cyclo M3 } & 2 & $5 / 13$ & 13.5 & 13.5 & 2 & 23.2 & 84.4 & 7.2 & 100 \\
\hline & & & & & & & $\begin{array}{r}\text { Avg. } \\
\text { StDev. }\end{array}$ & $\begin{array}{l}7.1 \\
0.1 \\
\end{array}$ & \\
\hline Cyclo M7 & 1 & $4 / 23$ & 13 & 12.5 & 2 & 24.2 & 68.5 & 8.3 & 100 \\
\hline \multirow[t]{2}{*}{ Cyclo M7 } & 3 & $5 / 13$ & 13.5 & 13.5 & 2 & 18.8 & 61.3 & 7.9 & 100 \\
\hline & & & & & & & $\begin{array}{r}\text { Avg. } \\
\text { StDev. }\end{array}$ & $\begin{array}{l}8.1 \\
0.3 \\
\end{array}$ & \\
\hline
\end{tabular}


Figure 10a. Biomass Concentration in CM1-1 Trial Runs.
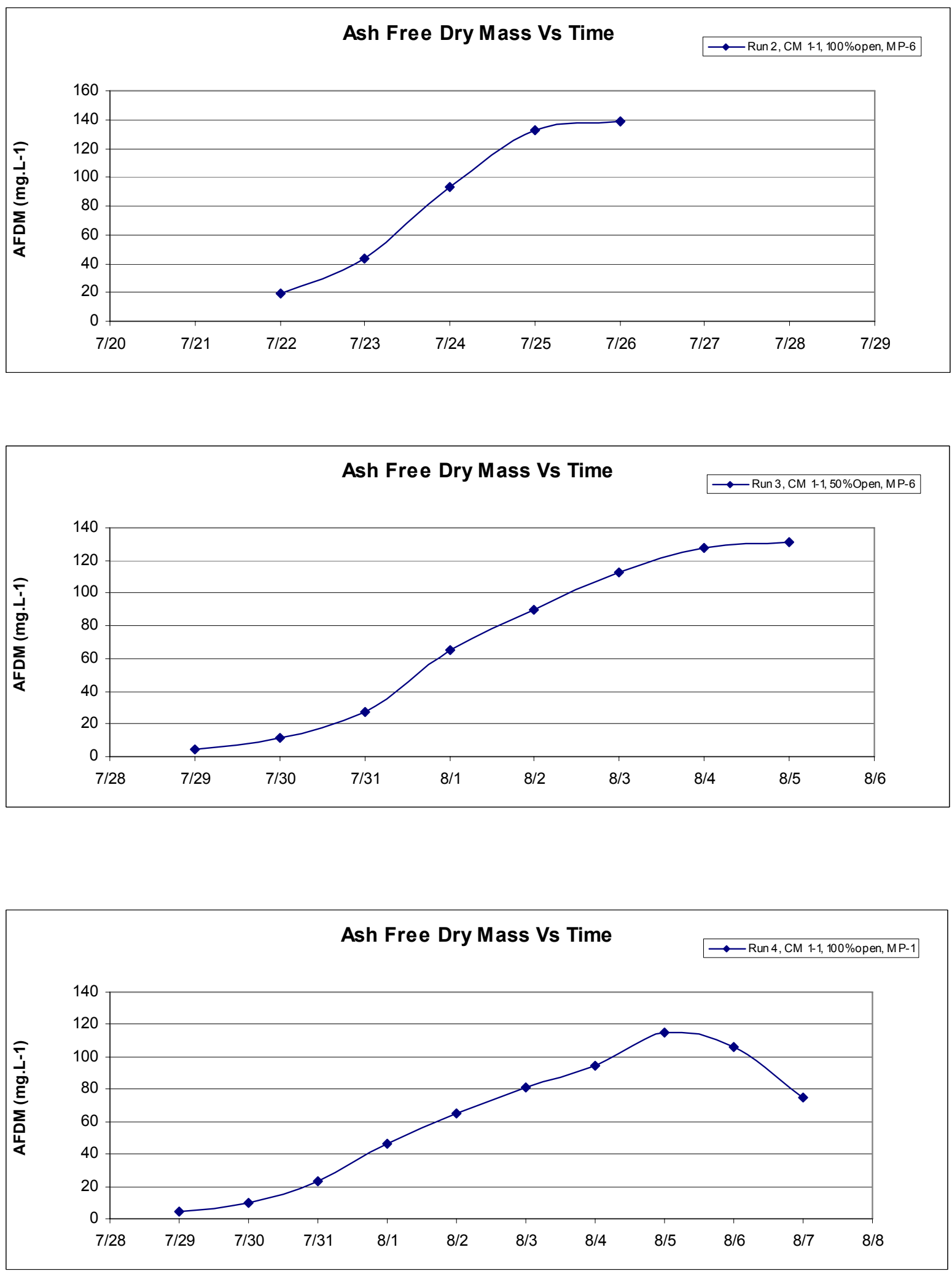
Figure 10b. Biomass Productivity of CM1-1 Trial Runs.
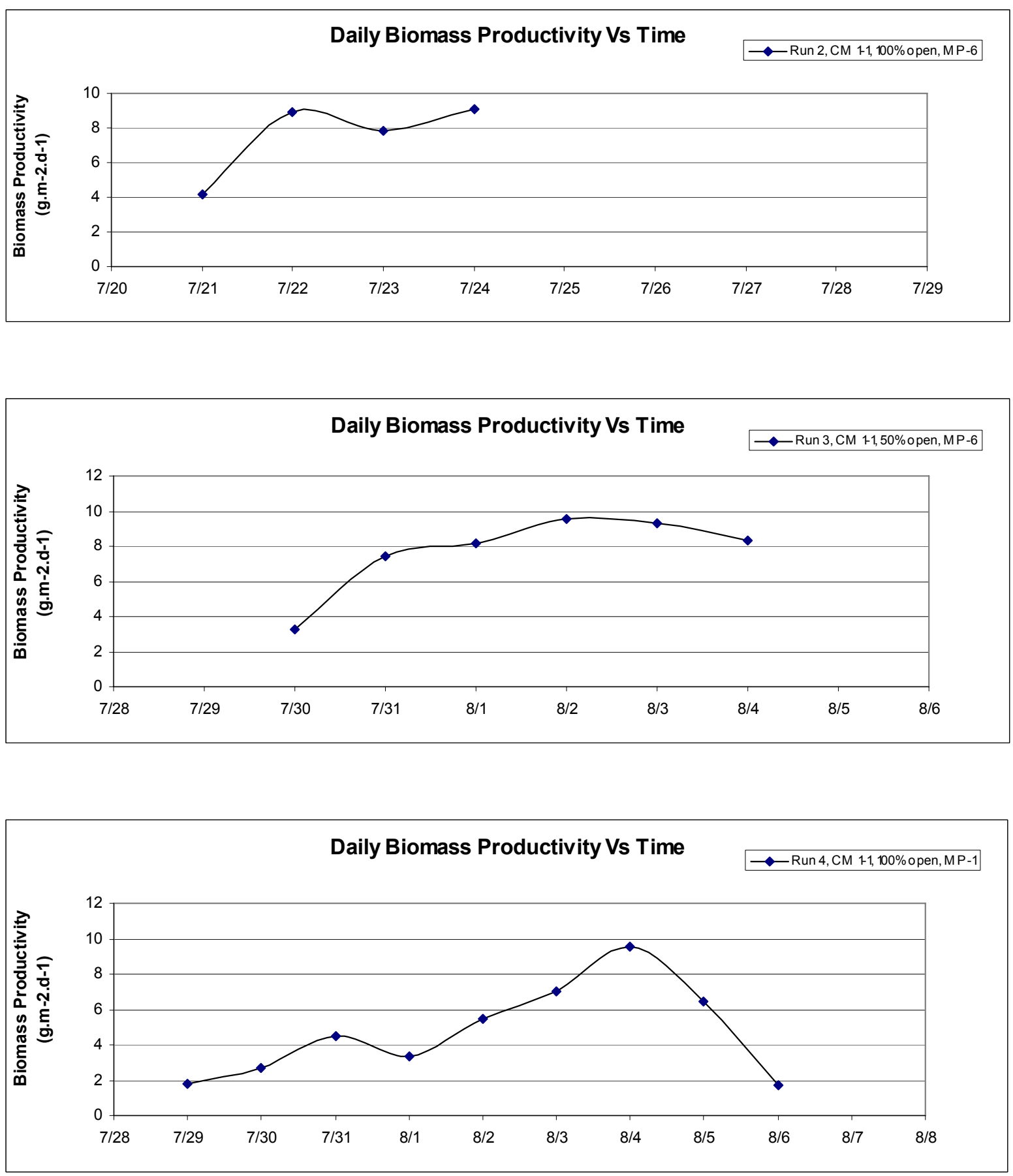


\subsection{Intermittent Illumination of RW Ponds}

In the Phase I of this project, it was found that when an open pond was partially covered, blocking out some of the incident light, the way in which the cover was placed determined how much the biomass productivity was reduced. A pond that was covered contiguously over $75 \%$ of its area produced $25 \%$ as much biomass as an uncovered pond, as would be expected. The PE on light incident to the culture suspension was therefore the same as for a totally uncovered pond. A pond that was entirely covered by a shade cloth that blocked $70 \%$ of the incident light produced about $60 \%$ as much as the uncovered pond, about twice as much as would be expected if the remaining light incident on the culture was used as the same efficiency. Its PE was thus higher, twice as high, because the incident intensity was lower. However, this result was also expected, as a shade cloth reduces incident light intensity and, thus, ameliorates the light saturation effect.

In a third experiment, a pond was covered $75 \%$ with opaque slats $(22.5 \mathrm{~cm}$ strips of thick, white polypropylene plastic) spaced $7.5 \mathrm{~cm}$ apart (thus allowing the culture to receive only $25 \%$ of the incident light intensity). Since the horizontal mixing speed was about $15 \mathrm{~cm} . \mathrm{s}-1$, the algal suspension (tough not the individual cells) cycled in and out of the light on an interval of $0.5 \mathrm{~s}$ and $1.5 \mathrm{~s}$, respectively. This pond also produced somewhat over $50 \%$ of the productivity of the uncovered pond, not $25 \%$ as expected. That is, its PE was also substantially higher, about twice that of the uncovered pond. Here though, the incident light intensity received by the cultures was not decreased over the open area and, thus, light saturation cannot explain this increase in relative productivity (per unit area of light received). The most logical explanation was that this was due to an amelioration of light inhibition. It appeared that cell suspensions subjected to the high light continuously (as in the completely open pond), or for longer periods than a few seconds (as in the $75 \%$ contiguously covered ponds) are inhibited in their photosynthetic efficiency. This inhibition could be relieved to some extent by exposing them only intermittently to the high light at relatively short cycle times (e.g. less than one second in the light).

These cycling times were much too long to be attributed to the flashing light effect first describe by Kok (1953), in which cells are exposed to the light for a few milliseconds and then are kept in the dark for five to ten times longer periods. A great deal of literature over the years has suggested that longer periodicities can increase photosynthetic conversion efficiencies (e.g. Laws et al., 1986, Laws and Berning, 1991). Thus, further investigation of this phenomenon was warranted.

We started by reproducing the general result from Phase I that intermittent illumination increased PE. Figure 11 shows some of the configurations used to intermittently cover ponds. Several such experiments were performed. A summary of the data is given in Tables 21 and 22. The duration of many of the experiments was restricted to short time periods due to excessive rainfall which either resulted in very low insolation, or overflowing of the ponds which made productivity measurements inaccurate. 
In the first two trials, $45 \%$ of the surface of one pond was covered by slats $15 \mathrm{~cm}$ wide, placed about $15 \mathrm{~cm}$ apart. The total coverage was not $50 \%$ because the slats could not be arranged without a remainder near the edge of the pond. The other pond was open. The biomass production from a pond with $55 \%$ of the light input was $80 \pm 2 \%$ of that from an uncovered pond. The corresponding PE was $46+2 \%$ greater (Table 22, Figure 12).

In the next experiment, the $15 \mathrm{~cm}$ slats were placed $7.5 \mathrm{~cm}$ apart, leaving only $33 \%$ of the pond open. Now the biomass production was two thirds of the open pond and the PE doubled. We then tried to determine whether the time in the light or the time out of the light was more important by comparing this arrangement $(15 \mathrm{~cm}$ slats, $7.5 \mathrm{~cm}$ apart, Figure 11) to arrangements for which about 50\% of the pond was left open (Figure11). In one case, the 15 $\mathrm{cm}$ slats were placed $15 \mathrm{~cm}$ apart. In the other case, $7.5 \mathrm{~cm}$ wide slats were placed $7.5 \mathrm{~cm}$ apart. So we now had two treatments with the suspensions exposed to the light for $7.5 \mathrm{~cm}$ (about $0.5 \mathrm{~s}$ ), but with different times in the dark ( $1 \mathrm{~s}$ versus $0.5 \mathrm{~s}$ ), and two treatments with exposure to the dark for $15 \mathrm{~cm}(1 \mathrm{~s})$, but different times in the light. Results are also given in Figure 13 and Tables 21, 22, 23. The length of time in the light or dark did not matter with about $50 \%$ coverage. With only 52 or $55 \%$ as much light input, the productivity was still $80 \%$ as much as an uncovered pond. PE was thus about $50 \%$ greater than the uncovered control. Comparing the 33\% open case to the $50+\%$ open cases, that is adding back roughly $50 \%$ more light (at high intensity), increased productivity only $15 \%$.

The analysis is blurred to some extent by the effects of diffuse light. The photosynthetic efficiencies for intermittently covered ponds were overestimated because the light intensity under the slats was not zero. It was estimated to about 5-8\% of the unobstructed intensity, based on a few individual measurements. The amount of light under the slats was minimized by having the slats as close to the pond surface as possible, by operating at greater depth. However, this was limited by the need for freeboard to hold rainwater. Therefore diffuse light still hit the pond surface under the slats. With 10-15\% more light impinging on the pond than calculated based on the open area, instead of expecting $50 \%$ of the productivity compared to a totally open pond, we would expect 60 to $65 \%$ as much, based strictly on total light input. The results, however, showed considerably more productivity, at about $80 \%$ of the open ponds, a significant effect.

The amount of diffuse light also depended on the width of the slats. The comparison of wide slats to narrow slats shown in Table 21 gives some idea of the magnitude of the error due to diffuse light under the slats. The light intensity under wide slats was less than that under narrow slats. In the two trials, the pond covered with narrow slats was $5 \%$ and $10 \%$ more productive than the one covered with wide slats.

In the experiments described in a subsequent section of this report, the light under the slats was measured continuously by putting another light probe at the water level under a typical slat. Ponds were operated deeper as well, $19 \mathrm{~cm}$ instead of $15 \mathrm{~cm}$, to minimize the gap between the slats and the pond surface. PE was thus more accurately calculated. The results were not materially different from the earlier ones. 
Doubling times of the algal cultures were measured for some runs when the suspensions were very dilute. The data given in Table 24 shows minimum doubling times for the different strains in totally open ponds. The J5 strain was cloned from the J strain by plating in the laboratory. It appeared to have a $10 \%$ longer minimum doubling time. In covered ponds, both strains grew about $15-20 \%$ more slowly when very dilute (Table 25). It made little difference whether the ponds $33 \%$ covered or $50 \%$ covered, as shown in Tables 26 . Side by side runs are compared in Table 27. In one run, the culture in the covered pond grew as fast as that in the uncovered pond. But in all other side trials, the same results were obtained: algal cultures in covered ponds grew $10-20 \%$ more slowly when dilute. So it appears that intermittent blocking out of light slows maximal growth rates to about the same extent no matter how much light is blocked. Why would this be so? One might expect the growth rate to be directly proportional to the amount of time spent in the light if the cells are always growing as fast as they can when in the light. However, when the cultures are very dilute the amount of light in the culture under the slats is grossly underestimated. Photons will travel to the (white painted) bottom of the ponds and scatter back up in all directions. So in this case, it is probably a poor assumption that the light is zero under the slats.

There are two important conclusions to be drawn from these experiments. First, they reveal circumstances when the PE of algal cultures under conditions of very high irradiance, are quite high. Second, the experiments provide a measure for photoinhibition under field conditions. 
Figure 11. Intermittently Covered Ponds.

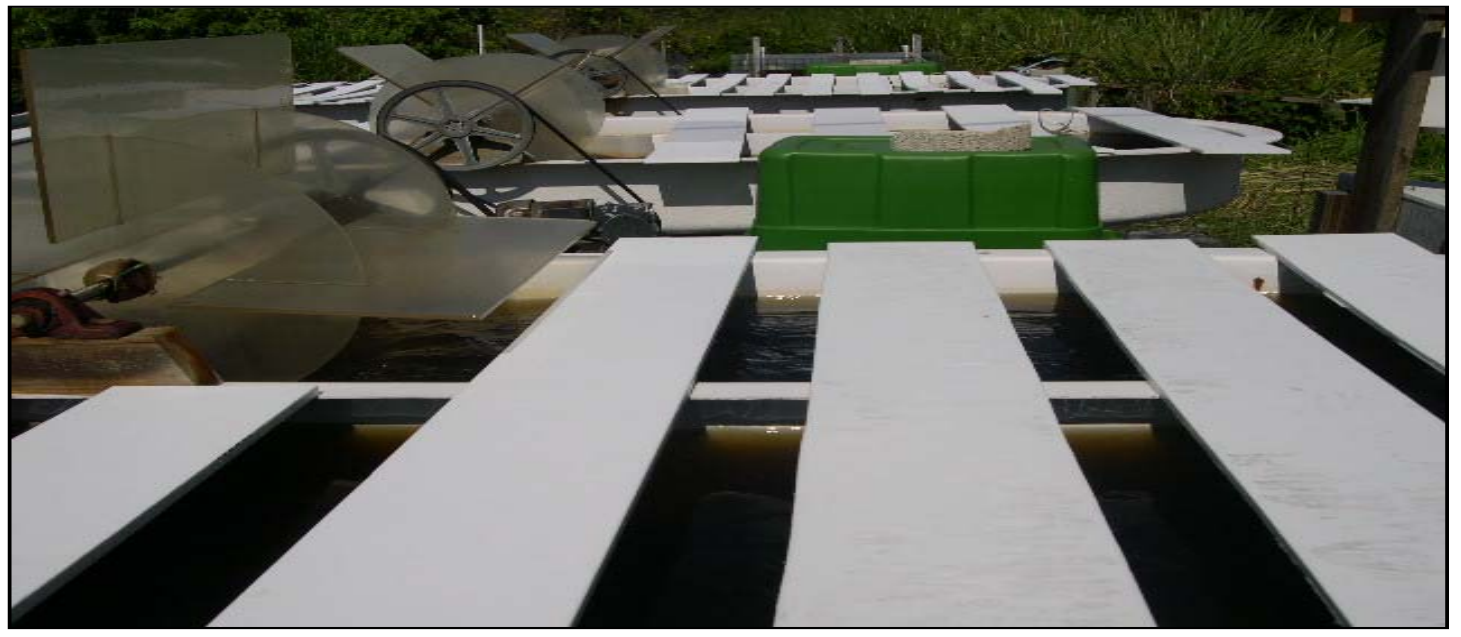

Interm ittently Illum inated $\mathrm{R}$ ace $\mathrm{w}$ a y $\mathrm{P}$ ond, $33 \%$ O pen A rea ( $15 \mathrm{~cm} \mathrm{w}$ ide slat, $7.5 \mathrm{~cm}$ wide gap)

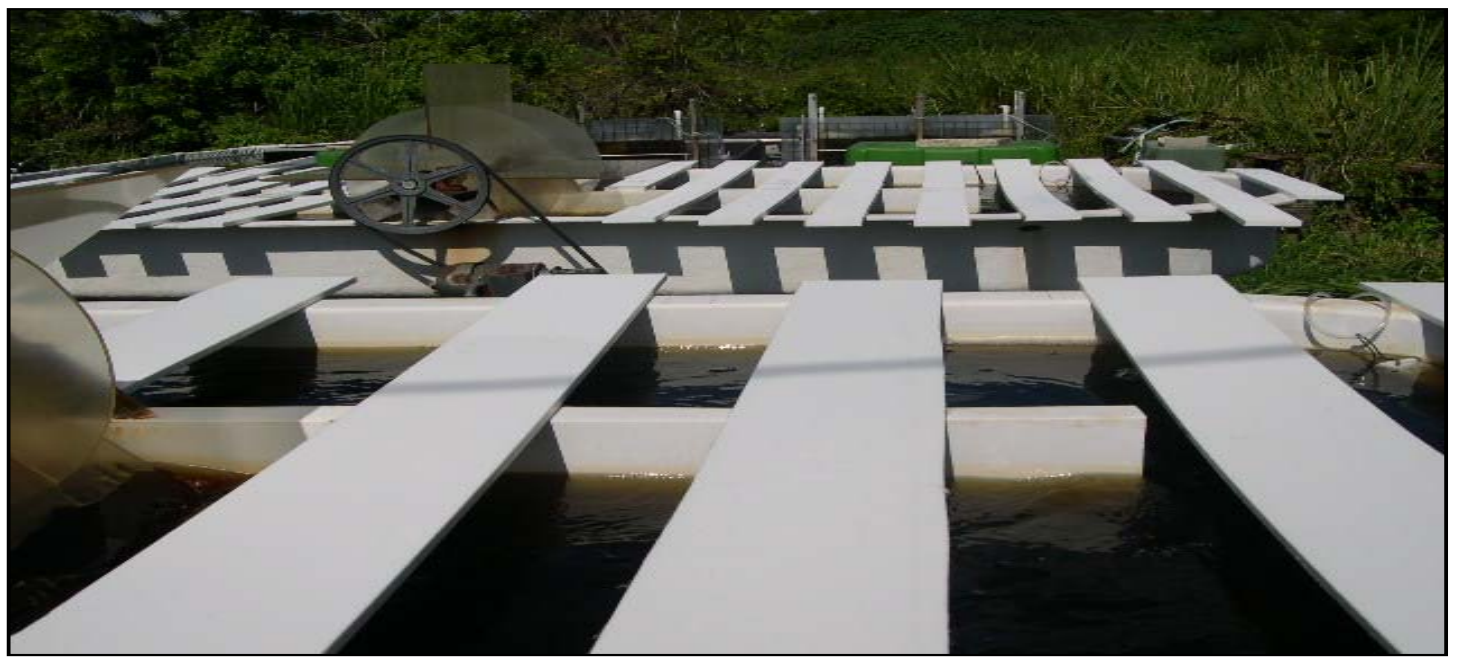

Interm ittently Illum inated $\mathrm{R}$ acew a $\mathrm{P}$ ond, $55 \%$ O pen A rea $(15 \mathrm{~cm}$ wide slat, $15 \mathrm{~cm}$ wide gap)

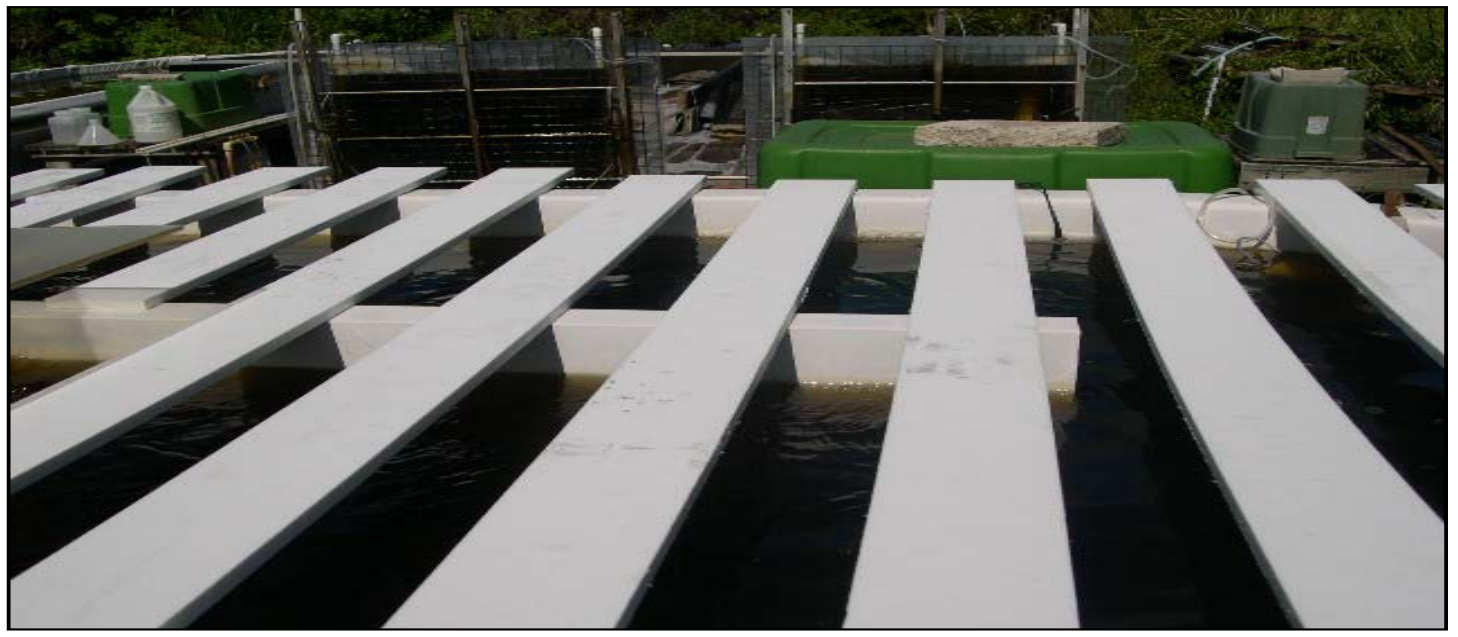

Interm ittently Illum inated R acew a y P ond, $52 \%$ O pen A rea

( $7.5 \mathrm{~cm}$ wide slat, $7.5 \mathrm{~cm}$ wide gap) 
Table 22.

\section{Slatted Pond Experiments Cyclotella J}

\begin{tabular}{|c|c|c|}
\hline & \multicolumn{2}{|c|}{$\begin{array}{c}\text { Ratio of Productivity and PE for slatted vs } \\
\text { Open Ponds }\end{array}$} \\
\hline $\begin{array}{c}\text { Dates of } \\
\text { Experiment }\end{array}$ & $50 \% / 100 \%$ & $33 \% / 100 \%$ \\
\hline $7 / 10-7 / 15 / 04$ & 0.791 .45 & \\
\hline $7 / 22-7 / 27 / 04$ & 0.801 .49 & \\
\hline $7 / 30-8 / 1 / 04$ & & 0.672 .06 \\
\hline $8 / 10-8 / 12 / 04$ & 0.801 .53 & 0.672 .14 \\
\hline
\end{tabular}


Figure 12. Open vs Slatted Ponds in Cyclotella sp (strain Cyclo J-5) in Small Ponds ( 2.8m-2) in Semi-continuous Culture.

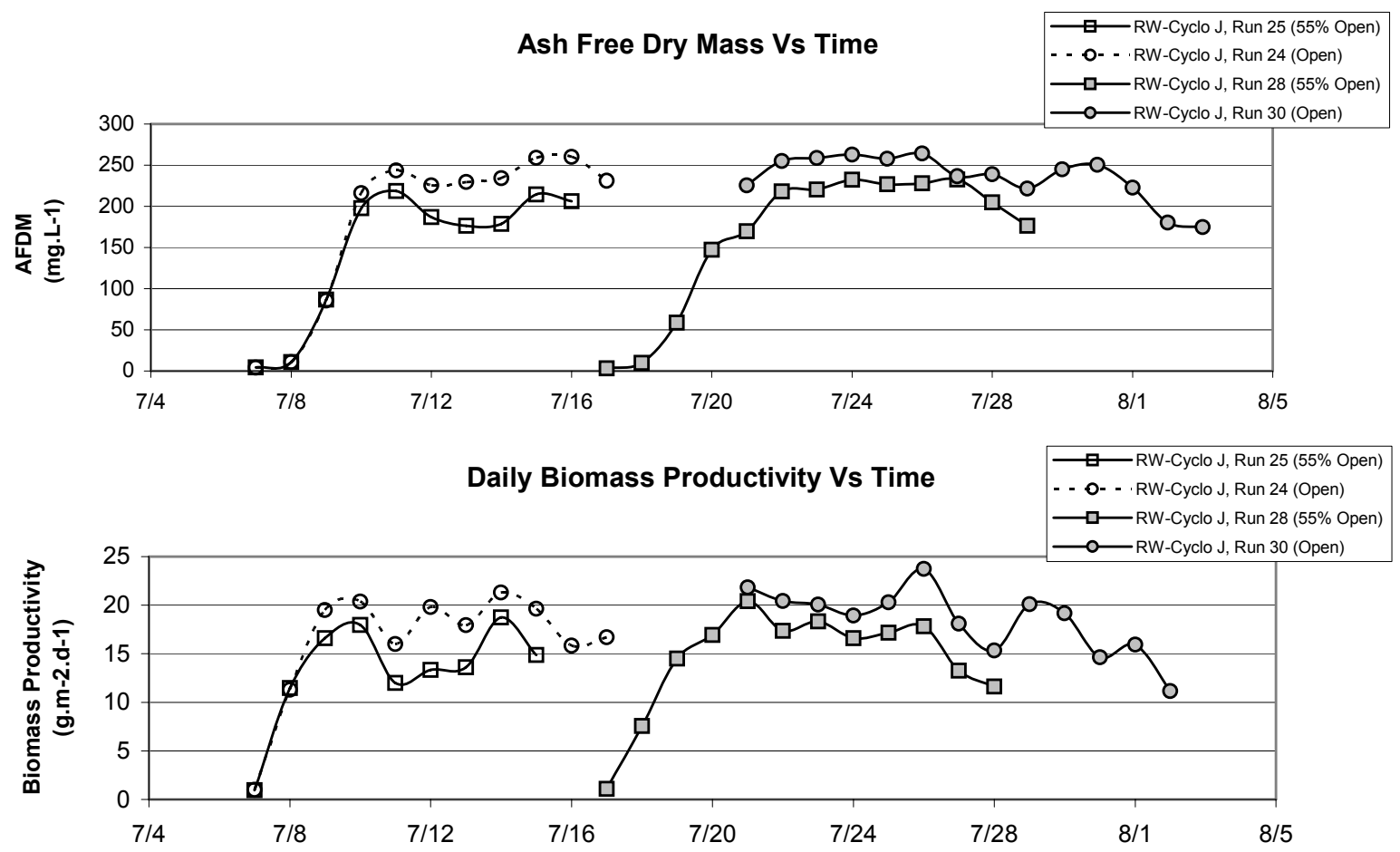

Pond Water Temperature Vs Time
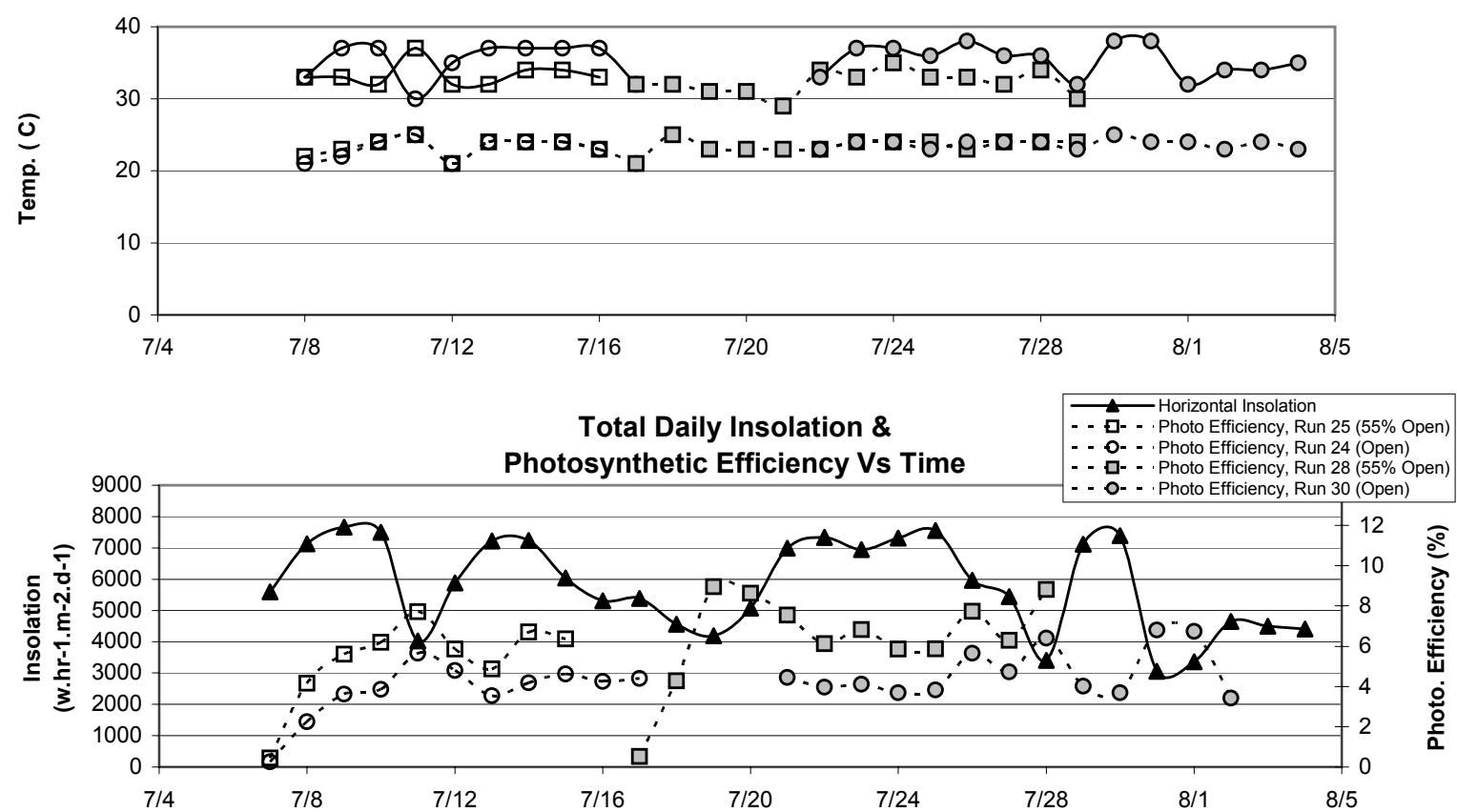
Figure 13. Open vs Slatted Ponds. Cyclotella sp (strain Cyclo J) in Small Ponds ( $2.8 \mathrm{~m}-2$ ) in Semi-continuous Culture.
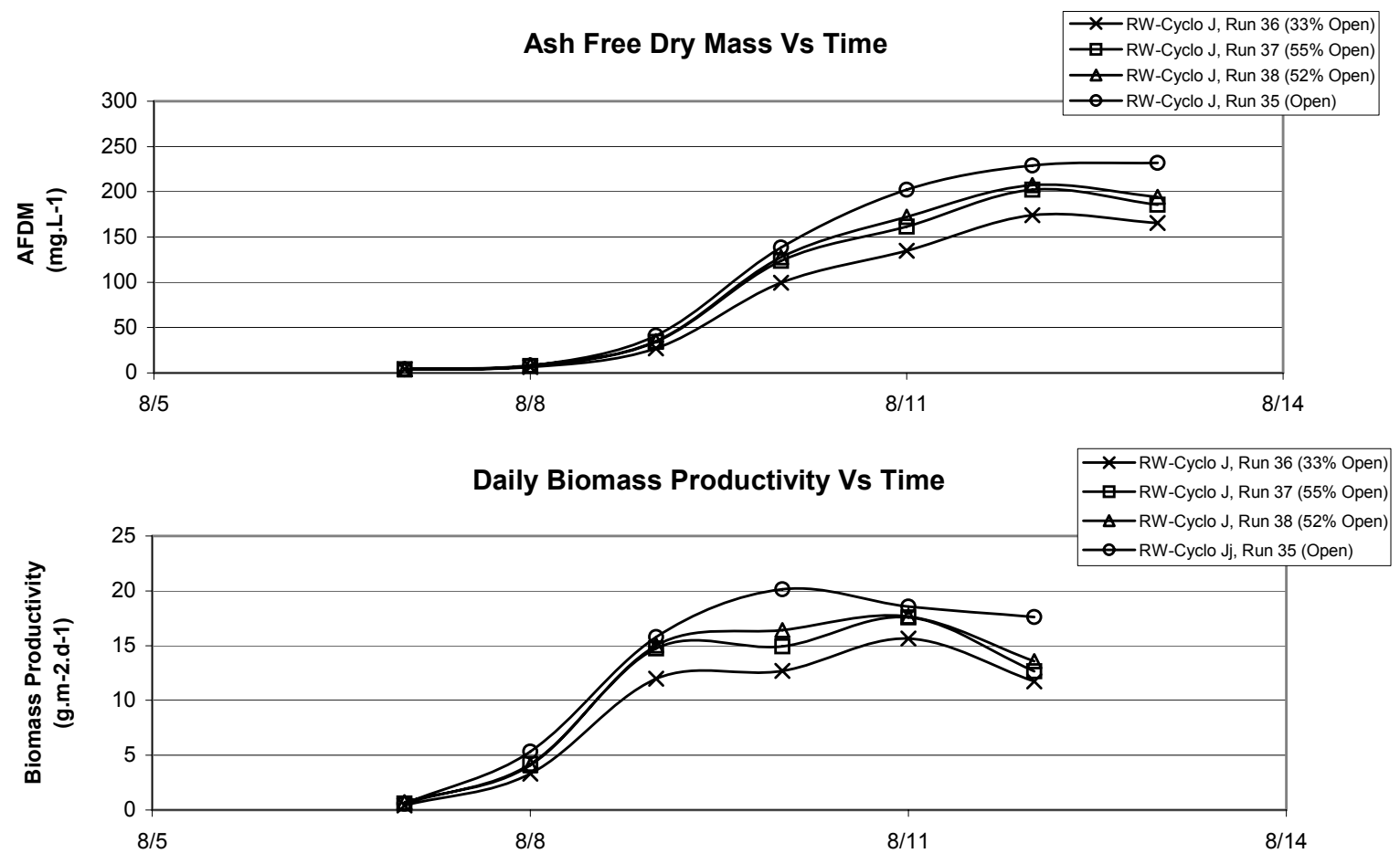

Pond Water Temperature Vs Time
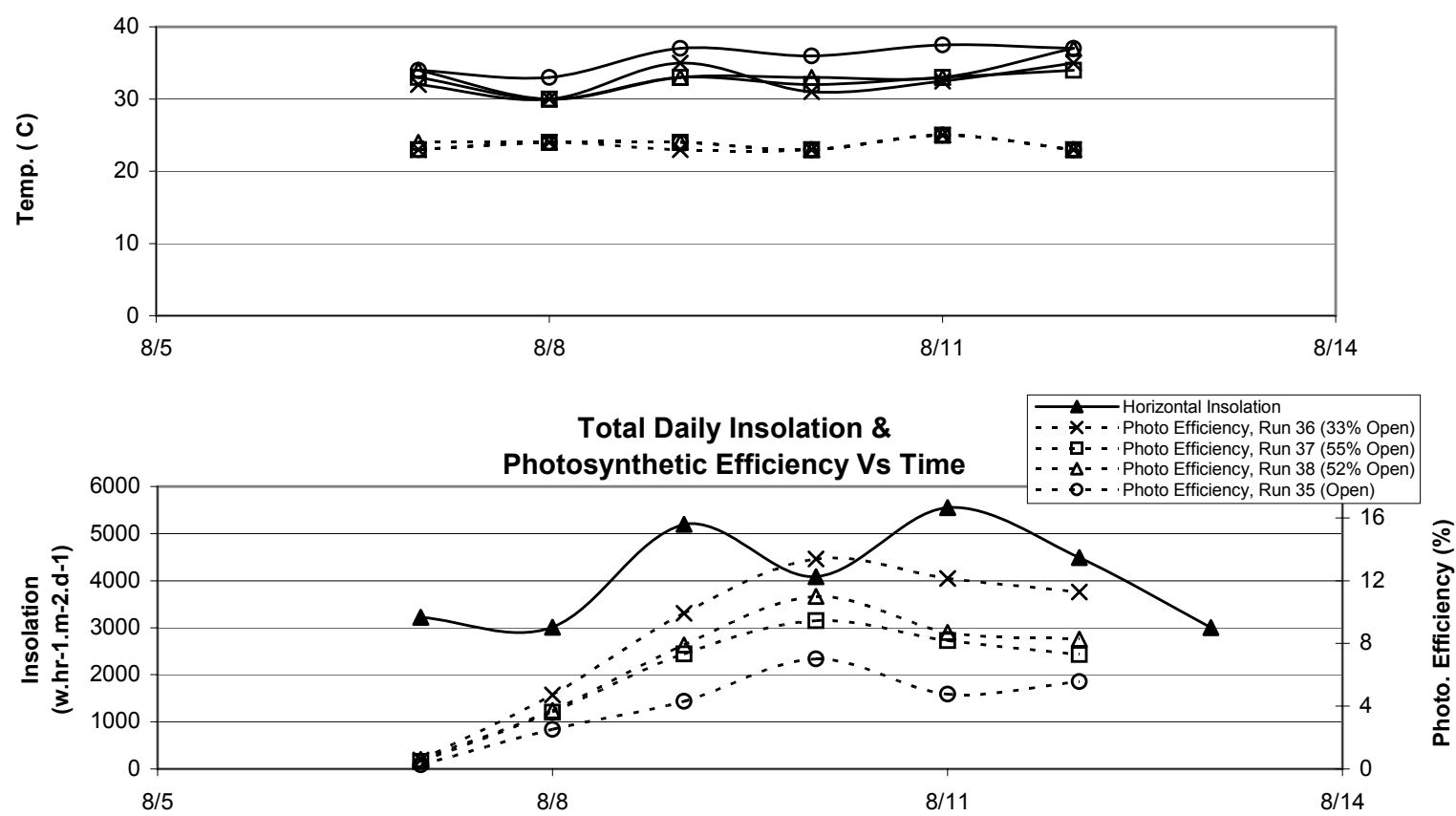
Table 23

Open vs Slatted Ponds. Cyclotella sp (strain Cyclo J)

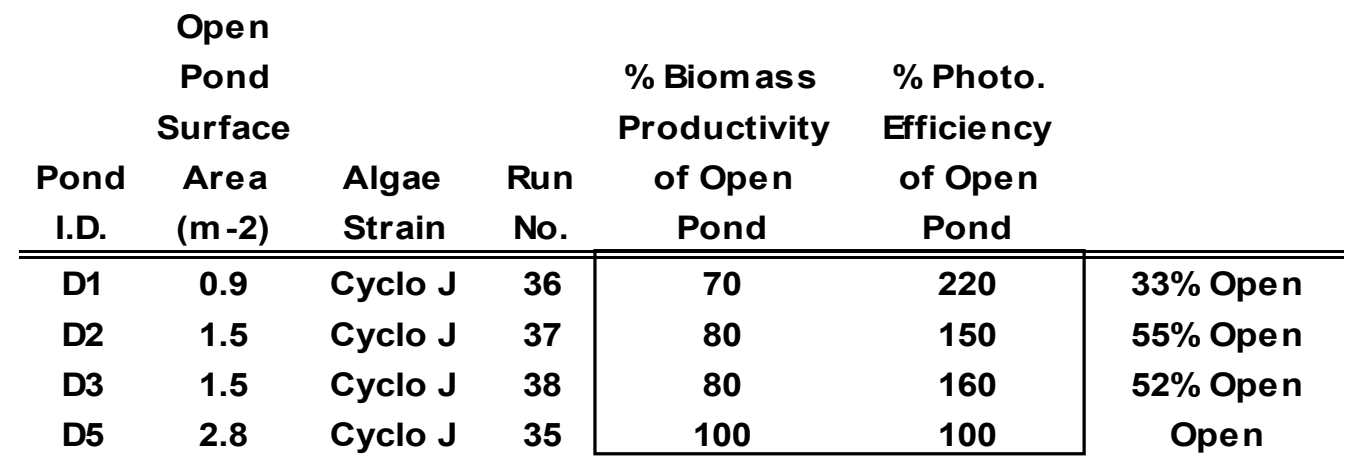


Table 24.

\section{Doubling}

\section{Times for Cyclotella sp (CycloJ \& J-5) in Open Small Ponds (2.8m-2)}

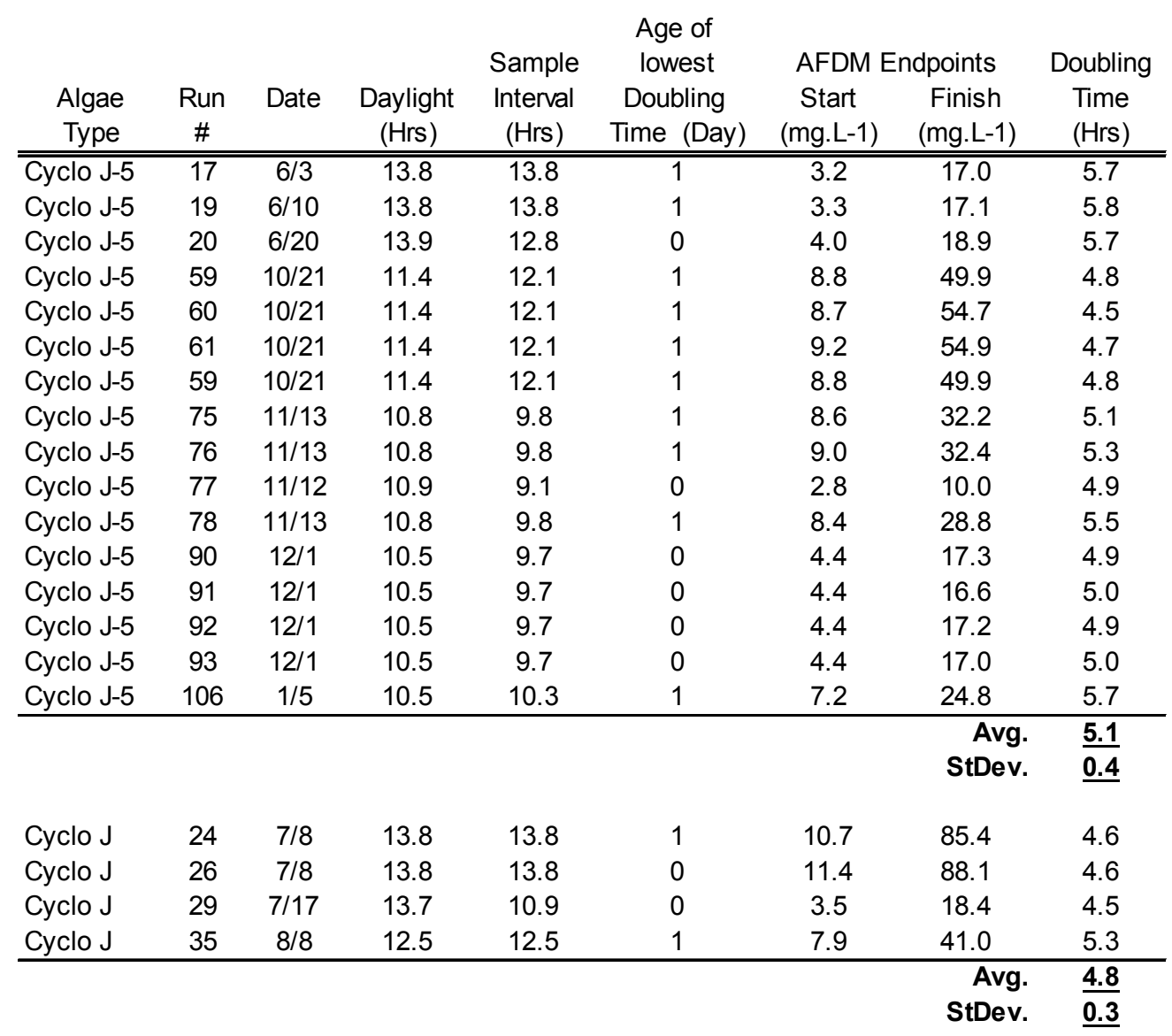


Table 25.

Doubling Times of Covered Ponds with CYCLO J5 and J

\begin{tabular}{|c|c|c|c|c|c|c|c|c|c|c|}
\hline & & & & & & & & & & $\begin{array}{c}\text { Slat - Gap } \\
\text { cm }\end{array}$ \\
\hline Cyclo J-5 & 18 & $6 / 9$ & 13.8 & 13.8 & 1 & 3.3 & 14.3 & 6.5 & 40 & \\
\hline Cyclo J-5 & 21 & $6 / 30$ & 13.9 & 16.4 & 1 & 11.3 & 57.9 & 5.8 & 34 & \\
\hline Cyclo J-5 & 22 & $6 / 30$ & 13.9 & 13.9 & 1 & 11.7 & 61.6 & 5.7 & 42 & \\
\hline & & & & & & & Avg. & $\underline{6.0}$ & & \\
\hline & & & & & & & StDev. & $\overline{0.4}$ & & \\
\hline Cyclo J & 25 & $7 / 8$ & 13.8 & 13.8 & 1 & 10.7 & 86.0 & 4.6 & 55 & $15-15$ \\
\hline Cyclo J & 28 & $7 / 18$ & 13.7 & 14.3 & 1 & 9.9 & 58.5 & 5.6 & 55 & $15-15$ \\
\hline Cyclo J & 34 & $7 / 31$ & 12.7 & 12.2 & 1 & 10.0 & 60.1 & 4.7 & 55 & $15-15$ \\
\hline Cyclo J & 37 & $8 / 8$ & 12.5 & 12.5 & 1 & 7.6 & 34.1 & 5.8 & 55 & $15-15$ \\
\hline Cyclo J & 38 & $8 / 8$ & 12.5 & 12.5 & 1 & 8.5 & 35.0 & 6.1 & 52 & 7.5 - 7.5 \\
\hline Cyclo J & 31 & $7 / 28$ & 12.8 & 13.1 & 1 & 14.3 & 77.3 & 5.3 & 33 & $15-7.5$ \\
\hline Cyclo J & 36 & $8 / 8$ & 12.5 & 12.5 & 1 & 6.4 & 27.1 & 6.0 & 33 & $15-7.5$ \\
\hline Cyclo J & 44 & $8 / 16$ & 13.1 & 9.6 & 0 & 12.6 & 42.3 & 5.5 & 52 & 7.5 - 7.5 \\
\hline Cyclo J & 41 & $8 / 16$ & 13.1 & 9.6 & 0 & 12.6 & 40.5 & 5.7 & 33 & $15-7.5$ \\
\hline Cyclo J & 43 & $8 / 16$ & 13.1 & 9.6 & 0 & 12.6 & 44.5 & 5.2 & 52 & 7.5 - 7.5 \\
\hline
\end{tabular}

Table 26.

Doubling Time and Slat Opening. Cyclotella J

\begin{tabular}{|c|c|c|c|}
\hline & $\begin{array}{l}15 \mathrm{~cm} \text { Slats } \\
15 \mathrm{~cm} \text { Gap }\end{array}$ & $\begin{array}{l}15 \mathrm{~cm} \text { Slats } \\
7.5 \mathrm{~cm} \text { Gap }\end{array}$ & $\begin{array}{l}7.5 \mathrm{~cm} \text { Slats } \\
7.5 \mathrm{~cm} \text { Gap } \\
\end{array}$ \\
\hline$\%$ Open Area & 55 & 33 & 50 \\
\hline Avg Doubling Time (hr) & 5.1 & 5.7 & 5.6 \\
\hline SD & 0.6 & 0.3 & 0.4 \\
\hline $\mathrm{n}$ & 4 & 3 & 3 \\
\hline
\end{tabular}


Table 27.

\section{Doubling Times of Covered Vs Uncovered Ponds with CYCLO J Side by Side}

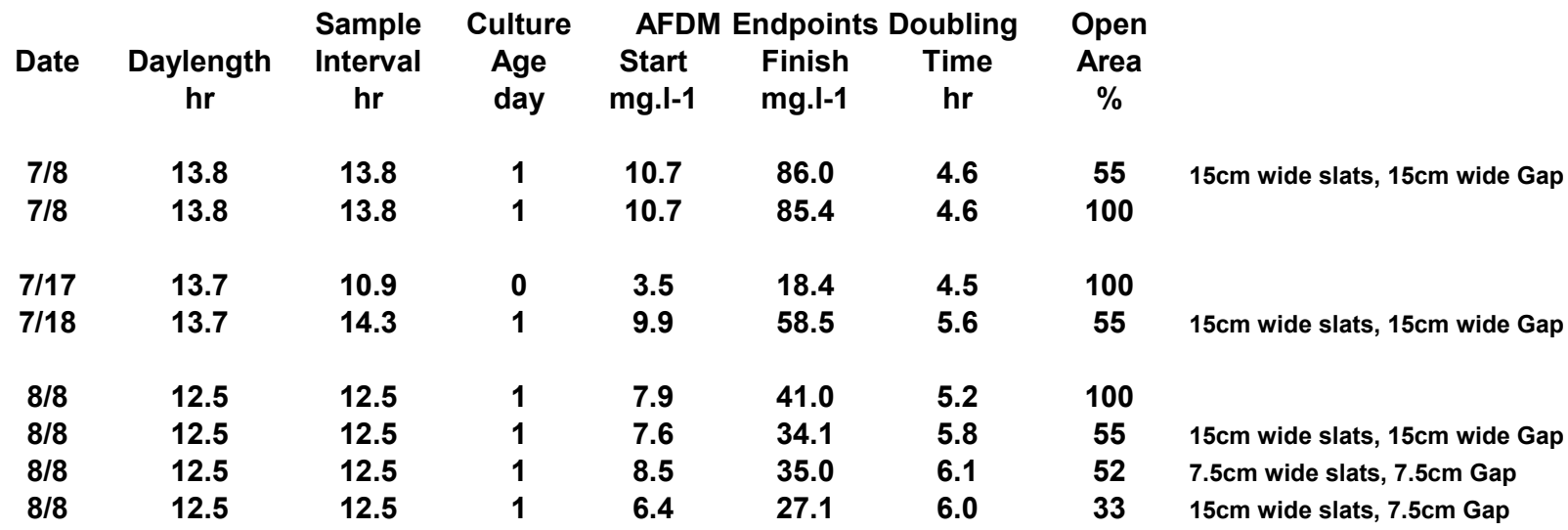




\subsection{Nitrogen Limited Productivity of Outdoor Raceway Ponds}

In outdoor cultivation of algal cultures, the greatest productivity is achieved when light is the input that limits production. But the utilization of light by algal cultures is complicated by the way in which light impinges on the cultures (through the surface), by the way in which individual cells respond to light input, and by the movement of the cells in and out of illuminated zones in the culture. The intermittent illumination experiments presented above show how complex the subject is. The nitrogen nutrition, and its interaction with the utilization of light, is another example of such complexity, and the potential for manipulating mass culture conditions to maximize productivity and/or photosynthetic efficiency.

Nitrogen is a macronutrient in cells, a major constituent of the amino acids in proteins. In photosynthetic cells, the pigments are arranged in large macromolecular structures containing many proteins. When algal cells are limited for nitrogen, they reduce their growth rate (e.g. cell division) and break down the photosynthetic apparatus, scavenging any nitrogen that becomes available from the degradation of the proteins associated with it. One very noticeable and rapid effect of nitrogen limitation in most algal strains, is their bleaching, due to a reduction in their pigment content. Another effect is a continued increase in biomass (eventually this is mostly due to increased cell size, rather than cell division) and redirecting a significant portion of their anabolic metabolism to the formation of non-protein molecules, specifically starch and/or lipids. This increased production of fermentable or extractable compounds under these conditions makes nitrogen limitation interesting from the standpoint of biofuels production.

It has been previously shown by the P.I. that microalgal batch cultures supplied with a limited amount of nitrogen, will grow (increase in biomass) well past their nitrogen sufficient cell quota (about $10 \% \mathrm{~N}$ ). Indeed, productivity of such $\mathrm{N}$-limited cultures can even exceed the maximum biomass productivity observed under $\mathrm{N}$ sufficiency. Of course, such a phenomenon is necessarily limited to a relatively short period in batch cultures, as after a burst of productivity at the onset of $\mathrm{N}$ limitation cell metabolism quickly shuts down. However, when cultures are diluted semi continuously, with some $\mathrm{N}$ added after each dilution, they could be operated for long periods of time under conditions of $\mathrm{N}$ limitation. The daily additions of limiting amounts of $\mathrm{N}$ lead to cycling in gross composition. The cells absorb the $\mathrm{N}$ quickly, forming protein. They then run out of the $\mathrm{N}$ in the medium and switch to forming storage products. Over the day, the cells go from very high rates of protein production to very high rates of carbohydrate production. Typically, cells will never contain a full 10\% $\mathrm{N}$ in their biomass under these conditions, but the $\mathrm{N}$ content does vary over the day. It has been consistently observed by the P.I. that biomass productivity does not diminish, and may even increase, when cultures are operated in this way. The amount of $\mathrm{N}$ added is crucial. Too much $\mathrm{N}$ and the cells are close to $\mathrm{N}$ sufficient, too little and $\mathrm{N}$ limitation, rather than light, limits productivity. Of course, light is also critical, as it determines the amount of $\mathrm{N}$ that the cells could use before they become limited.

Limiting the amount of $\mathrm{N}$ available to the algal cells may be expected to lead to increased production if it results in a specific lowering of the light harvesting pigment content, rather than a breakdown of the entire photosynthetic apparatus, including reactions centers. Thus $\mathrm{N}$ 
limited cultures may exhibit, at least transitionally, the decreased antenna size phenotype desired for the mutants tested (but not observed) in the above experiments, and thus would exhibit a higher photosynthetic efficiency. Of course, if the decrease in pigment content occurs proportionally in both the reaction centers and antenna pigments, no increased photosynthetic efficiency would be anticipated.

Several trials were run in which the ponds were operated semi continuously, as usual, but in which some of the units were fed less nitrogen. A control was run, with enough $\mathrm{N}$ to make the cells $\mathrm{N}$-sufficient (maximum $\mathrm{N}$ content of about 10\%), along with a middle level of $\mathrm{N}$ input to lower the $\mathrm{N}$ content to about $5 \%$, and a low level to essentially bring the cells to their lowest possible $\mathrm{N}$ content.

We did not have the laboratory capability to measure either the residual $\mathrm{N}$ in the medium or the $\mathrm{N}$ content of the biomass. In order to evaluate the level of $\mathrm{N}$ nutrition, the daily and cumulative biomass production was compared to the daily and cumulative $\mathrm{N}$ inputs. The parameter that was calculated was the per cent of the nitrogen algal growth potential (NAGP) that was achieved for each treatment. It was assumed that the normal, $\mathrm{N}$ sufficient cell quota for $\mathrm{N}$ was 0.1 , that is, normally one can produce ten times as much $\mathrm{N}$ sufficient biomass as the amount of $\mathrm{N}$ added. (This may be somewhat of an underestimate, with $\mathrm{N}$ contents of 8 to $10 \%$ typical under sufficient conditions, but this does not change the arguments). For each run in each trial, a table was made to asses the extent of $\mathrm{N}$ limitation using this parameter. Table 28 shows the parameters, their definition, and the formulas used to calculate them. Table 29 is an example of such a tabulation. Basically, the total cumulative N-AGP, after dilution and nitrogen addition on the morning of a given day, is: the total of all nitrogen inputs (on that day and all previous days), plus the amount of biomass left in the pond after dilution, plus the total amount of biomass that has been harvested on all days, minus the amount of nitrogen growth potential in the residual nitrogen (that did not get incorporated into biomass) that has been removed with dilution on all days. For computational purposes, the formulas used to keep track of this material balance, and presented in the tables, are recursive. We were careful to keep the other nutrients at sufficient levels.

Four trials were run. The first was unsuccessful, too many of the cultures failed. It is sometimes difficult to determine how to add nutrients to outdoor cultures because the variability in the weather results in algal growth potential that may change daily, and because there is a several day delay in the feedback from the dry weight analyses as to the actual production. One is guessing how much nutrient to add each day. The problem is even more challenging with $\mathrm{Si}$, since adding too much causes the cells to clump together. It only takes a small excess for this to happen. When it does, the clumping of cells leads to decline of the culture, usually accompanied by an increase in predatory amoeba (see also prior discussion).

For the remaining trials, a summary table is presented showing the states of nitrogen nutrition (Tables 30-32). These tables contain a daily log of the amount of N added, the N-AGP and cumulative N-AGP, the daily biomass productivity, and the cumulative biomass productivity, and the per cent utilization of the added $\mathrm{N}$ (assuming a 10\% content of $\mathrm{N}$ ). On any given day, a culture is nitrogen sufficient if this per cent is $100 \pm 20$. As this per cent gets higher 
and higher, the culture is more and more nitrogen limited. From the last column, the nitrogen content of the biomass can be estimated. If the total cumulative biomass production divided by the total N-AGP available (the total cumulative N-AGP) is $100 \%$ or less, then the assumption is that the biomass is $\mathrm{N}$ sufficient and the $\mathrm{N}$ content is 0.1 . Otherwise it is 0.1 times 100 divided the value shown as per cent total cumulative N-AGP. Thus if the value of cumulative biomass production divided by total cumulative N-AGP is $200 \%$, the biomass nitrogen content is 0.05 (5\%).

Figures 14a and b show the ponds and algal samples from the ponds for Trial 4. The results of each of the trials are shown in Figures 15,16,17 and summarized in Tables 33 and 34. A quick perusal of the summary tables show that even considerable nitrogen deficiency does not greatly impact biomass productivity, and in some cases even augments it. The middle level of $\mathrm{N}$ input in each trial was noticeably gold or tan in color (less fucoxanthin) compared to the coffee brown controls. In these trials we were not able to demonstrate significant increases in biomass productivity in the cultures with less pigment. These cultures were however stable, and were cleaner in terms of cell debris and cell surface debris. For instance, the control pond in Trial 4 had to be restarted (from the middle dose $\mathrm{N}$ pond) because it developed clumps and declined in productivity. The modestly $\mathrm{N}$ deficient culture continued to grow stably.

All of the trials were initiated by starting with a small inoculum into a medium with some initial urea-N. Cultures were operated in batch until they reached a density of about 150 mg.l-1. In some trials this amount of algal growth was already equal to or greater than the NAGP. In others the ponds had to be diluted a few days before this occurred.

For Trial 2 dilution was started on 10/22. The control pond used about $50 \%$ of the added N. The middle dose pond (1.5 g.m-2 of $\mathrm{N}$ added per day) was also close to nitrogen sufficiency. The color of this pond was usually dark coffee, but sometimes a bit dull brown indicating some nitrogen deficiency. 1.5 g.m-2.d-1 of $\mathrm{N}$ supports about 15 g.m-2.d-1 of biomass which was the average productivity expected and measured. However, we measured approximately $1.7 \mathrm{mg} . \mathrm{l}-1$ of nitrate- $\mathrm{N}$ in the incoming seawater. This yields 0.08 g.m-2.d-1 N at 33\% dilution per day, adding another 0.8 g.m-2.d-1 to the AGP. This amount is only significant at the lowest level of nitrogen added ( 0.5 gN.m-2.d-1). This pond produced about 2.5 times the algal biomass that would be predicted from the N-AGP calculated on the basis of a nitrogen content of $10 \%$ of the biomass. The $\mathrm{N}$ content of the biomass was thus about $4 \%$. The culture lost most of its pigment and appeared golden-yellow instead of dark brown. But biomass production was hardly impacted.

Statistically there was no difference between any pairs of ponds to the 0.05 level except between the highest and lowest levels of $\mathrm{N}$ addition (Table 35). In this case, the most $\mathrm{N}$ limited culture had the highest biomass production even to the 0.01 confidence level. However, the results were still quite close and not of practical significance except that the $\mathrm{N}$ limited cultures were not less productive than the sufficient cultures.

All of the cultures decreased in density towards the end of the experiment. The cells were clean in all runs with no evidence of bacterial growth on the surfaces. Low $\mathrm{N}$ cells had very 
little color, with no pigmented inclusion bodies. The highest $\mathrm{N}$ cells had pigmented inclusion bodies, as did the middle $\mathrm{N}$ cells, although the pigmentation was clearly less. This would indicate that at the middle dose, $\mathrm{N}$ deficiency was incipient but not far along.

Another experimental trial was done, Trial 3, but at lower $\mathrm{N}$ doses. Now the middle dose from before became the control, $\mathrm{N}$ sufficient dose at $1.5 \mathrm{gm}-2 \mathrm{~d}-1 \mathrm{~N}$ with the other ponds at 1.0 and 0.5 respectively. The middle dose culture in this experiment crashed mid way through the run, leaving only three good days of data to compare it to the higher dose. But the highest and lowest dosed cultures were stable for many days. In any case, the results were very similar to the previous trial. There was again a statistically higher biomass production in the lowest $\mathrm{N}$ pond compared to the highest $\mathrm{N}$ level pond over an eleven day period.

Yet another trial was run (Trial 4), with the objective to keep the experiment going as long as possible to gain a sense of how stable the $\mathrm{N}$ limited cultures can be. Since the weather was getting colder, the $\mathrm{N}$ levels were again decreased to accommodate the expected lower biomass productivities. The high level culture received $1.5 \mathrm{gm}-2 \mathrm{~N}$ when it was fed fully, the middle level was 0.5 , and this time a culture was run with no $\mathrm{N}$ added except for that in the saltwater $(0.08 \mathrm{gNm}-2 \mathrm{~d}-1)$. A fourth pond was operated as a batch to determine the $\mathrm{N}$ AGP on the nitrogen in the saltwater (Figure 18). The batch culture yielded $100 \mathrm{mgl}-1$ of biomass, calculated to contain only about $1.7 \% \mathrm{~N}$ the first time it was run. However, it was uncovered, allowing insects to drop into it, a potential source of $\mathrm{N}$. A second trial was run, but this time the pond was covered. It yielded only about $60 \mathrm{mgl}-1$ of biomass, calculated to contain $2.8 \% \mathrm{~N}$. The culture was pale yellow. The productivity of the semi continuously culture operated without added $\mathrm{N}$ was from 0 to $4 \mathrm{gm}-2 \mathrm{~d}-1$, after the initial added $\mathrm{N}$ was used. As in the other trials, there was almost no difference between the productivities of the pond with $\mathrm{N}$ sufficient biomass and the pond with less than half the amount of $\mathrm{N}$ in the biomass.

The results with these N-limited cultures are important because they demonstrate that alteration of the proximate composition of the algal biomass, through nitrogen limitation to yield more valuable energy products (lipids or fermentable carbohydrates) can be accomplished without sacrificing productivity. In fact it even may increase productivity somewhat, as observed in some experiments and, more dramatically, in prior laboratory work by the P.I. 
Table 28.

\title{
Formulas used in Determining Nutrient Limitation
}

\author{
$\mathbf{N}-\mathbf{Q}_{c}=$ Algal Cell Quota of $\mathrm{N}$ in $\mathrm{g}=10$ \\ $\mathbf{N}_{\text {sw }}(\mathbf{g})=$ Amt of N present in S.W. used in culture media and dilutions (in g) \\ $N_{S W D=0}=($ Volume of SW used to top of pond to running depth, in $L) X(N$ content of S.W., in g.L-1) \\ $N_{S W ~ D=1 \ldots . . X}=($ Dilution Volume in $L) X(N$ content in S.W. in g. L-1 ) \\ Dilution Volume in $\mathrm{L}=($ Begining depth in $\mathrm{cm}$ - Lowered Depth in $\mathrm{cm}) \times 29.03 \mathrm{~L} / \mathrm{cm}$ ( D-pond measurement made in Fall '03) \\ $\mathrm{N}$ content in SW additions in $\mathrm{g} / \mathrm{I}=(\mathrm{N}$ content of SW used in media in $\mathrm{mg} / \mathrm{L} / 1000)$ \\ N-AGP INPUTS D $_{\text {(g.m-2) }}=\mathrm{N}-\mathrm{AGP}_{\mathrm{UREA} D}+\mathrm{N}-\mathrm{AGP}_{\mathrm{SW} D}+\mathrm{N}-\mathrm{AGP}_{\mathrm{FW}}$ \\ $\mathrm{N}-A G P_{\mathrm{FW}}$ - Considered to be negligible due to small volume added during top off (up to $14.5 \mathrm{~L}$ of $\mathrm{FW}$ ). \\ N-AGPUREA D (g.m -2) = Algal growth potential of Urea-nitrogen from that day's feeding. \\ $\mathrm{N}-\mathrm{AGP}_{\text {UREA }}=$ ( urea addition in $\mathrm{g} X \%$ Nitrogen in Urea $X \mathrm{~N}-\mathrm{Q}_{\mathrm{C}}$ ) / surface area of pond in $\mathrm{m}-2$ \\ N-AGP SW D $_{\text {(g.m -2) }}=$ Algal Growth Potential of Nitrogen present in SW used for culture media and dilutions. \\ $\mathrm{N}-\mathrm{AGP}_{\mathrm{SW}}=\left(\mathrm{N}\right.$ in SW $\left.X \mathrm{~N}-\mathrm{Q}_{\mathrm{C}}\right) /$ surface area of Pond in $\mathrm{m}-2$

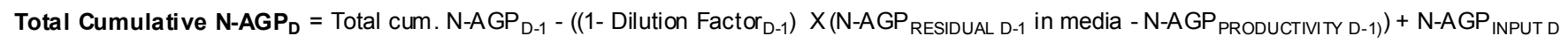 \\ Dilution Factor $_{D}=\left(\right.$ Lowered depth $_{D} /$ Beginning Depth $\left._{D}\right)$ \\ N-AGP RESIDUAL D $=\left(\mathrm{N}-\mathrm{AGP}_{\text {RESIDUAL D-1 }} \mathrm{X}\left(\right.\right.$ Dilution Factor $\left.\left._{\mathrm{D}-1}\right)\right)+\left(\mathrm{N}-\mathrm{AGP}_{\text {INPUTS D-1 }}-\mathrm{N}-\mathrm{AGP}_{\text {PRODUCTIVITY D-1 }}\right)$ \\ $\mathrm{N}-\mathrm{AGP}_{\mathrm{PRODUCTVITY}} \mathrm{D}-1=\left(\right.$ Daily biomass Productivity $\left._{\mathrm{D}-1}\right)$ \\ $N-A G P_{\text {INPUT D }}=\left(N-A G P_{\text {UREA D }}+\mathrm{N}-\mathrm{AGP}_{\text {SW D }}\right)$ \\ N content of Salt Water (g.L-1) $=0.0017$ \\ Si content of Salt Water (g.L-1) $=0.005$
}


Table 29.

\section{Nitrogen Limitation of Cyclotella sp (strain Cyclo J-5) in Semi-continuous Culture.}

\begin{tabular}{|c|c|c|c|c|c|c|c|c|c|c|}
\hline Date & $\begin{array}{c}\text { Daily } \\
\text { N-Urea } \\
\text { Feeding } \\
\text { (g) }\end{array}$ & $\begin{array}{c}\text { AFDM } \\
\text { (mg.L-1) }\end{array}$ & $\begin{array}{l}\text { Dilution } \\
\text { Factor }\end{array}$ & $\begin{array}{c}\text { Daily } \\
\text { Now }_{\text {sw }} \\
\text { Addition } \\
\text { (g) }\end{array}$ & $\begin{array}{c}\text { Day's } \\
\text { NINPUTS-AGP }^{\text {(g.m-2) }}\end{array}$ & $\begin{array}{l}N_{\text {RESIDUAL }} \\
\text { AGP in } \\
\text { Media } \\
\text { (g.m-2) }\end{array}$ & $\begin{array}{c}\text { Total } \\
\text { Cumulative } \\
\text { N-AGP } \\
\text { (g.m-2) }\end{array}$ & $\begin{array}{c}\text { Daily } \\
\text { Biomass } \\
\text { Productivity } \\
\text { (g.m-2.d-1) }\end{array}$ & $\begin{array}{c}\text { Cumulative } \\
\text { Productivity } \\
\text { (g.m-2) }\end{array}$ & $\begin{array}{c}\% \text { Total N-AGP. } \\
\text { Obsenved }\end{array}$ \\
\hline \multicolumn{11}{|c|}{ Trial 2: D2-Run 61, 0.5g.m-2 N (Low Dose), 33\% Dilution. } \\
\hline $10 / 20$ & 1.5 & 2.81 & 1.000 & 0.73 & 5.07 & 0.00 & 5.07 & 1.27 & 1.27 & $25 \%$ \\
\hline $10 / 21$ & 9.0 & 9.21 & 1.000 & 0.00 & 14.79 & 3.80 & 19.85 & 7.69 & 8.96 & $45 \%$ \\
\hline $10 / 22$ & 3.0 & 54.91 & 1.000 & $0 . \infty$ & 4.93 & 10.89 & 24.78 & 18.37 & 27.33 & $110 \%$ \\
\hline $10 / 23$ & 3.0 & 158.60 & 0.429 & 0.00 & 4.93 & 0.00 & 29.71 & 17.00 & 44.32 & $149 \%$ \\
\hline $10 / 24$ & 3.0 & 192.60 & 0.500 & 0.49 & 6.69 & 0.00 & 36.40 & 24.93 & 69.25 & $190 \%$ \\
\hline $10 / 25$ & 3.0 & 262.50 & 0.500 & 0.37 & 6.25 & 0.00 & 42.65 & 17.55 & 86.80 & $204 \%$ \\
\hline $10 / 26$ & 3.0 & 237.19 & 0.494 & 0.37 & 6.25 & 0.00 & 48.90 & 16.69 & 103.49 & $212 \%$ \\
\hline $10 / 27$ & 3.0 & 233.80 & 0.500 & 0.39 & 6.33 & 0.00 & 55.23 & 16.28 & 119.77 & $217 \%$ \\
\hline $10 / 28$ & 3.0 & 225.40 & 0.500 & 0.37 & 6.25 & 0.00 & 61.48 & 17.89 & 137.65 & $224 \%$ \\
\hline $10 / 29$ & 3.0 & 231.95 & 0.500 & 0.37 & 6.25 & 0.00 & 67.73 & 17.49 & 155.15 & $229 \%$ \\
\hline $10 / 30$ & 3.0 & 232.60 & 0.500 & 0.37 & 6.25 & 0.00 & 73.99 & 18.59 & 173.74 & $235 \%$ \\
\hline $10 / 31$ & 3.0 & 228.10 & 0.456 & 0.37 & 6.25 & 0.00 & 80.24 & 19.38 & 193.12 & $241 \%$ \\
\hline $11 / 1$ & 3.0 & 238.70 & 0.500 & 0.42 & 6.44 & 0.00 & 86.68 & 12.33 & 205.45 & $237 \%$ \\
\hline $11 / 2$ & 3.0 & 201.52 & 0.500 & 0.37 & 6.25 & 0.00 & 92.93 & 16.06 & 221.51 & $238 \%$ \\
\hline $11 / 3$ & 3.0 & 207.84 & 0.500 & 0.37 & 6.25 & 0.00 & 99.18 & 14.81 & 236.32 & $238 \%$ \\
\hline $11 / 4$ & 3.0 & 202.65 & 0.500 & 0.37 & 6.25 & 0.00 & 105.43 & 12.68 & 249.00 & $236 \%$ \\
\hline $11 / 5$ & 3.0 & 185.85 & 0.500 & 0.37 & 6.25 & 0.00 & 111.68 & 7.15 & 256.15 & $229 \%$ \\
\hline $11 / 6$ & 3.0 & 140.60 & 0.500 & 0.37 & 6.25 & 0.00 & 117.93 & 14.94 & 271.09 & $230 \%$ \\
\hline $11 / 7$ & 3.0 & 169.90 & 0.500 & 0.37 & 6.25 & 0.00 & 124.18 & 14.42 & 285.51 & $230 \%$ \\
\hline $11 / 8$ & 3.0 & 181.05 & 0.500 & 0.37 & 6.25 & 0.00 & 130.43 & 10.78 & 296.29 & $227 \%$ \\
\hline $11 / 9$ & 3.0 & 162.41 & 0.500 & 0.37 & 6.25 & 0.00 & 136.68 & 9.81 & 306.10 & $224 \%$ \\
\hline
\end{tabular}

Pond volume $=429 \mathrm{~L}$. Ntrogen concentration of Salt Water $=1.7 \mathrm{mg} . \mathrm{L}-1$. 
Table 30.

\section{Nitrogen Limitation of Cyclotella sp (strain Cyclo J-5) in Semi-continuous Culture}

Summary Table for Trial 2

\begin{tabular}{|c|c|c|c|c|c|c|c|c|c|}
\hline \multirow{5}{*}{ Date } & \multicolumn{3}{|c|}{ D5-Run $59,3.0 \mathrm{~g} . \mathrm{m}-2 \mathrm{~N}$} & \multicolumn{3}{|c|}{ D1-Run $60,1.5 \mathrm{~g} . \mathrm{m}-2 \mathrm{~N}$} & \multicolumn{3}{|c|}{ D2-Run $61,0.5 \mathrm{~g} . \mathrm{m}-2 \mathrm{~N}$} \\
\hline & Total & Cumulative & & Total & Cumulative & & Total & Cumulative & \\
\hline & Cumulative & Biomass & $\%$ Total & Cumulative & Biomass & $\%$ Total & Cumulative & Biomass & $\%$ Total \\
\hline & N-AGP & Production & Cumulative & N-AGP & Production & Cumulative & N-AGP & Production & Cumulative \\
\hline & (g.m-2) & (g.m-2) & $\mathrm{N}-\mathrm{AGP}$ & (g.m-2) & (g.m-2) & $\mathrm{N}-\mathrm{AGP}$ & $(g \cdot m-2)$ & (g.m-2) & N-AGP \\
\hline $10 / 20$ & 17.39 & 1.19 & $7 \%$ & 10.00 & 1.15 & $11 \%$ & 5.07 & 1.27 & $25 \%$ \\
\hline $10 / 21$ & 32.18 & 8.51 & $26 \%$ & 24.78 & 9.19 & $37 \%$ & 19.85 & 8.96 & $45 \%$ \\
\hline $10 / 22$ & 61.75 & 26.13 & $42 \%$ & 39.57 & 27.90 & $71 \%$ & 24.78 & 27.33 & $110 \%$ \\
\hline $10 / 23$ & 87.75 & 40.45 & $46 \%$ & 56.22 & 45.64 & $81 \%$ & 29.71 & 44.32 & $149 \%$ \\
\hline $10 / 24$ & 108.51 & 57.16 & $53 \%$ & 72.33 & 65.62 & $91 \%$ & 36.40 & 69.25 & $190 \%$ \\
\hline $10 / 25$ & 132.80 & 71.22 & $54 \%$ & 88.44 & 82.28 & $93 \%$ & 42.65 & 86.80 & $204 \%$ \\
\hline $10 / 26$ & 155.76 & 87.28 & $56 \%$ & 104.63 & 100.33 & $96 \%$ & 48.90 & 103.49 & $212 \%$ \\
\hline $10 / 27$ & 178.92 & 100.95 & $56 \%$ & 120.73 & 117.24 & $97 \%$ & 55.23 & 119.77 & $217 \%$ \\
\hline $10 / 28$ & 201.41 & 115.52 & $57 \%$ & 136.84 & 133.93 & $98 \%$ & 61.48 & 137.65 & $224 \%$ \\
\hline $10 / 29$ & 223.32 & 130.40 & $58 \%$ & 152.95 & 150.56 & $98 \%$ & 67.73 & 155.15 & $229 \%$ \\
\hline $10 / 30$ & 245.36 & 145.85 & $59 \%$ & 169.06 & 165.38 & $98 \%$ & 73.99 & 173.74 & $235 \%$ \\
\hline $10 / 31$ & 267.82 & 163.34 & $61 \%$ & 185.16 & 177.00 & $96 \%$ & 80.24 & 193.12 & $241 \%$ \\
\hline $11 / 1$ & 291.66 & 176. 16 & $60 \%$ & 201.29 & 186.02 & $92 \%$ & 86.68 & 205.45 & $237 \%$ \\
\hline $11 / 2$ & 314.36 & 191.35 & $61 \%$ & 217.40 & 199.18 & $92 \%$ & 92.93 & 221.51 & $238 \%$ \\
\hline $11 / 3$ & 336.51 & 205.39 & $61 \%$ & 233.50 & 213.05 & $91 \%$ & 99.18 & 236. 32 & $238 \%$ \\
\hline $11 / 4$ & 358.40 & 220.15 & $61 \%$ & 249.61 & 228.32 & $91 \%$ & 105.43 & 249.00 & $236 \%$ \\
\hline $11 / 5$ & 380.24 & 228.20 & $60 \%$ & 265.72 & 237.26 & $89 \%$ & 111.68 & 256.15 & $229 \%$ \\
\hline $11 / 6$ & 398.87 & 242.91 & $61 \%$ & 281.84 & 253.22 & $90 \%$ & 117.93 & 271.09 & $230 \%$ \\
\hline $11 / 7$ & 417.56 & 257.65 & $62 \%$ & 297.99 & 271.81 & $91 \%$ & 124.18 & 285.51 & $230 \%$ \\
\hline $11 / 8$ & 437.95 & 269.73 & $62 \%$ & 314.09 & 287.60 & $92 \%$ & 130.43 & 296.29 & $227 \%$ \\
\hline $11 / 9$ & 457.86 & 277.77 & $61 \%$ & 330.20 & 300.77 & $91 \%$ & 136.68 & 306.10 & $224 \%$ \\
\hline
\end{tabular}


Table 31.

\section{Nitrogen Limitation of Cyclotella sp (strain Cyclo J-5) in Semi-continuous Culture}

\section{Summary Table for Trial 3}

\begin{tabular}{|c|c|c|c|c|c|c|c|c|c|}
\hline \multirow{4}{*}{ Date } & \multicolumn{3}{|c|}{ D3-Run $78,1.5 \mathrm{~g} . \mathrm{m}-2 \mathrm{~N}$} & \multicolumn{3}{|c|}{ D1-Run $76,1.0$ g. m-2 N } & \multicolumn{3}{|c|}{ D2-Run $77,0.5 \mathrm{~g} . \mathrm{m}-2 \mathrm{~N}$} \\
\hline & Total & Cumulative & & Total & Cumulative & & Total & Cumulative & \\
\hline & Cumulative & Biomass & $\%$ Total & Cumulative & Biomass & $\%$ Total & Cumulative & Biomass & $\%$ Total \\
\hline & $\begin{array}{l}\text { N-AGP } \\
\text { (g.m-2) }\end{array}$ & $\begin{array}{c}\text { Production } \\
\text { (g.m-2) }\end{array}$ & $\begin{array}{c}\text { Cumulative } \\
\mathrm{N}-\mathrm{AGP}\end{array}$ & $\begin{array}{l}\text { N-AGP } \\
\text { (g.m-2) }\end{array}$ & $\begin{array}{c}\text { Production } \\
\text { (g. m-2) }\end{array}$ & $\begin{array}{c}\text { Cumulative } \\
\text { N-AGP }\end{array}$ & $\begin{array}{l}\text { N-AGP } \\
\text { (g.m-2) }\end{array}$ & $\begin{array}{l}\text { Production } \\
\text { (g.m-2) }\end{array}$ & $\begin{array}{c}\text { Cumulative } \\
\text { N-AGP }\end{array}$ \\
\hline $11 / 12$ & 17.39 & 0.84 & $5 \%$ & 17.39 & 0.94 & $5 \%$ & 17.39 & 1.08 & $6 \%$ \\
\hline $11 / 13$ & 32.18 & 3.91 & $12 \%$ & 32.18 & 4.45 & $14 \%$ & 32.18 & 4.67 & $15 \%$ \\
\hline $11 / 14$ & 46.96 & 12.73 & $27 \%$ & 42.03 & 13.51 & $32 \%$ & 37.10 & 14.21 & $38 \%$ \\
\hline $11 / 15$ & 55.26 & 24.61 & $45 \%$ & 45.67 & 27.08 & $59 \%$ & 36.04 & 26.68 & $74 \%$ \\
\hline $11 / 16$ & 63.48 & 35.80 & $56 \%$ & 51.42 & 37.52 & $73 \%$ & 38.38 & 39.60 & $103 \%$ \\
\hline $11 / 17$ & 74.21 & 44.50 & $60 \%$ & 60.54 & 46.82 & $77 \%$ & 44.19 & 49.09 & $111 \%$ \\
\hline $11 / 18$ & 85.64 & 51.71 & $60 \%$ & 70.88 & 53.81 & $76 \%$ & 50.02 & 56.61 & $113 \%$ \\
\hline $11 / 19$ & 96.61 & 65.11 & $67 \%$ & 81.12 & 67.32 & $83 \%$ & 55.81 & 72.34 & $130 \%$ \\
\hline $11 / 20$ & 109.17 & 79.07 & $72 \%$ & 91.86 & 82.49 & $90 \%$ & 61.62 & 87.14 & $141 \%$ \\
\hline $11 / 21$ & 123.69 & 94.81 & $77 \%$ & 102.60 & 98.36 & $96 \%$ & 67.43 & 106.67 & $158 \%$ \\
\hline $11 / 22$ & 139.35 & 108.37 & $78 \%$ & 113.34 & 113.37 & $100 \%$ & 73.24 & 121.49 & $166 \%$ \\
\hline $11 / 23$ & 155.02 & 120.51 & $78 \%$ & 124.07 & 124.88 & $101 \%$ & 79.05 & 135. 10 & $171 \%$ \\
\hline $11 / 24$ & 170.69 & 131.46 & $77 \%$ & 134.83 & 130.78 & $97 \%$ & 84.89 & 146.85 & $173 \%$ \\
\hline $11 / 25$ & 186.35 & 143.41 & $77 \%$ & 145.57 & 139.02 & $95 \%$ & 90.70 & 161.34 & $178 \%$ \\
\hline $11 / 26$ & 202.11 & 155.35 & $77 \%$ & & & & 96.60 & 170.45 & $176 \%$ \\
\hline $11 / 27$ & 217.78 & 165.75 & $76 \%$ & & & & 102.41 & 182.74 & $178 \%$ \\
\hline $11 / 28$ & 233.29 & 180.37 & $77 \%$ & & & & 108.22 & 193.39 & $179 \%$ \\
\hline $11 / 29$ & 248.96 & 190.29 & $76 \%$ & & & & 114.03 & 201.44 & $177 \%$ \\
\hline
\end{tabular}


Table 32.

\section{Nitrogen Limitation of Cyclotella sp (strain Cyclo J-5) in Semi-continuous Culture}

Summary Table for Trial 4

\begin{tabular}{|c|c|c|c|c|c|c|}
\hline \multirow[b]{4}{*}{ Date } & \multicolumn{3}{|c|}{ D1-Run $116,1.5 \mathrm{~g} . \mathrm{m}-2 \mathrm{~N}$} & \multicolumn{3}{|c|}{ D2-Run $117,0.5 \mathrm{~g} . \mathrm{m}-2 \mathrm{~N}$} \\
\hline & Total & Cumulative & & Total & Cumulative & \\
\hline & Cumulative & Biomass & $\%$ Total & Cumulative & Biomass & $\%$ Total \\
\hline & $\begin{array}{l}\text { N-AGP } \\
\text { (g.m-2) }\end{array}$ & $\begin{array}{c}\text { Production } \\
\text { (g. m-2) }\end{array}$ & $\begin{array}{l}\text { Cumulative } \\
\text { N-AGP }\end{array}$ & $\begin{array}{l}\text { N-AGP } \\
\text { (g.m-2) }\end{array}$ & $\begin{array}{c}\text { Production } \\
\text { (g.m-2) }\end{array}$ & $\begin{array}{c}\text { Cumulative } \\
\mathrm{N}-\mathrm{AGP}\end{array}$ \\
\hline $1 / 19$ & 11.22 & 1.24 & $11 \%$ & 10.00 & 0.99 & $10 \%$ \\
\hline $1 / 20$ & 18.62 & 2.12 & $11 \%$ & 17.39 & 1.81 & $10 \%$ \\
\hline $1 / 21$ & 18.62 & 6.26 & $34 \%$ & 17.39 & 5.67 & $33 \%$ \\
\hline $1 / 22$ & 18.62 & 13.94 & $75 \%$ & 17.39 & 12.27 & $71 \%$ \\
\hline $1 / 23$ & 18.62 & 21.30 & $114 \%$ & 17.39 & 21.09 & $121 \%$ \\
\hline $1 / 24$ & 18.62 & 25.94 & $139 \%$ & 17.39 & 25.58 & $147 \%$ \\
\hline $1 / 25$ & 21.08 & 31.45 & $149 \%$ & 19.85 & 29.90 & $151 \%$ \\
\hline $1 / 26$ & 37.16 & 40.36 & $109 \%$ & 23.17 & 39.88 & $172 \%$ \\
\hline $1 / 27$ & 53.24 & 49.74 & $93 \%$ & 26.49 & 49.79 & $188 \%$ \\
\hline $1 / 28$ & 69.33 & 54.55 & $79 \%$ & 29.81 & 55.27 & $185 \%$ \\
\hline $1 / 29$ & 75.79 & 64.22 & $85 \%$ & 33.12 & 65.69 & $198 \%$ \\
\hline $1 / 30$ & 88.79 & 77.45 & $87 \%$ & 36.44 & 78.78 & $216 \%$ \\
\hline $1 / 31$ & 104.87 & 88.13 & $84 \%$ & 39.76 & 88.68 & $223 \%$ \\
\hline $2 / 1$ & 120.95 & 98.79 & $82 \%$ & 43.08 & 99.13 & $230 \%$ \\
\hline $2 / 2$ & 129.03 & 106.26 & $82 \%$ & 46.39 & 106.98 & $231 \%$ \\
\hline $2 / 3$ & 143.03 & 117.26 & $82 \%$ & 49.71 & 118.07 & $238 \%$ \\
\hline $2 / 4$ & 151.71 & 127.22 & $84 \%$ & 53.03 & 124.11 & $234 \%$ \\
\hline $2 / 5$ & 167.12 & 134.03 & $80 \%$ & 56.35 & 128.61 & $228 \%$ \\
\hline
\end{tabular}

\begin{tabular}{|c|c|c|c|c|c|c|}
\hline \multirow[b]{4}{*}{ Date } & \multicolumn{3}{|c|}{ D1-Run $120,1.5 \mathrm{~g} \cdot \mathrm{m}-2 \mathrm{~N}$} & \multicolumn{3}{|c|}{ D2-Run $117,0.5 \mathrm{~g} \cdot \mathrm{m}-2 \mathrm{~N}$} \\
\hline & Total & Cumulative & & Total & Cumulative & \\
\hline & Cumulative & Biomass & $\%$ Total & Cumulative & Biomass & $\%$ Total \\
\hline & $\begin{array}{l}\text { N-AGP } \\
\text { (q. m-2) }\end{array}$ & $\begin{array}{l}\text { Production } \\
\text { (q. m-2) }\end{array}$ & $\begin{array}{c}\text { Cumulative } \\
\text { N-AGP }\end{array}$ & $\begin{array}{l}\text { N-AGP } \\
\text { (q. } m-2 \text { ) }\end{array}$ & $\begin{array}{l}\text { Production } \\
\text { (q. m-2) }\end{array}$ & $\begin{array}{c}\text { Cumulative } \\
\text { N-AGP }\end{array}$ \\
\hline $2 / 7$ & 16.55 & 0.00 & $0 \%$ & 62.91 & 147.77 & $235 \%$ \\
\hline $2 / 8$ & 31.33 & 12.22 & $39 \%$ & 66.23 & 157.44 & $238 \%$ \\
\hline $2 / 9$ & 46.97 & 22.63 & $48 \%$ & 69.55 & 167.50 & $241 \%$ \\
\hline $2 / 10$ & 59.71 & 33.42 & $56 \%$ & 72.86 & 175.91 & $241 \%$ \\
\hline $2 / 11$ & 72.95 & 38.91 & $53 \%$ & 76.18 & 179.33 & $235 \%$ \\
\hline $2 / 12$ & 69.17 & 45.07 & $65 \%$ & 76.18 & 185.04 & $243 \%$ \\
\hline $2 / 13$ & 76.57 & 50.04 & $65 \%$ & 78.65 & 191.93 & $244 \%$ \\
\hline $2 / 14$ & 92.21 & 57.13 & $62 \%$ & 81.96 & 201.42 & $246 \%$ \\
\hline 2/15 & 104.33 & 69.41 & $67 \%$ & 85.28 & 211.94 & $249 \%$ \\
\hline 2/16 & 117.30 & 80.60 & $69 \%$ & 88.60 & 222.22 & $251 \%$ \\
\hline
\end{tabular}


Figure 14a.

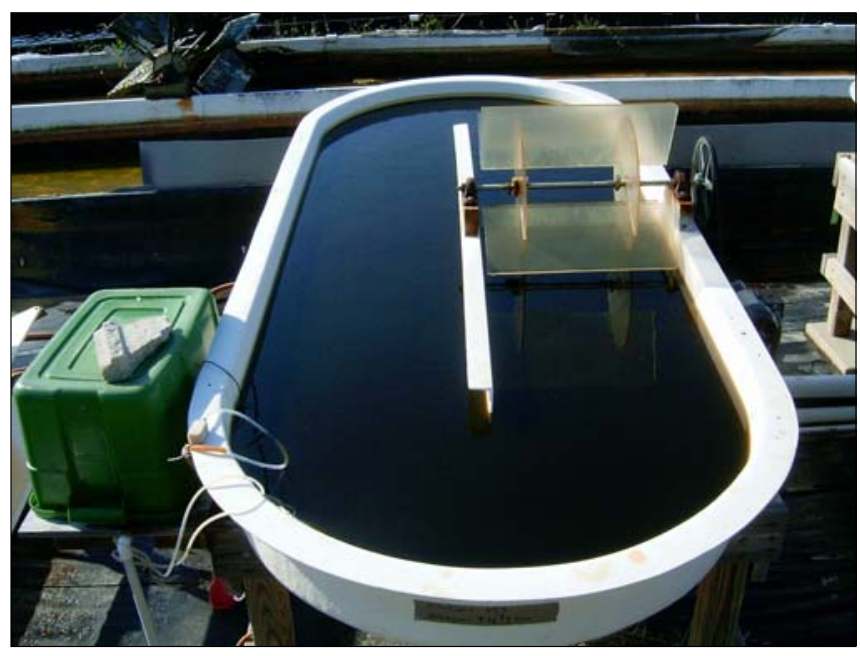

Trial 4. D1-Run 120 (1.5g.m-2 N) $\mathrm{N}$-Sufficient Pond

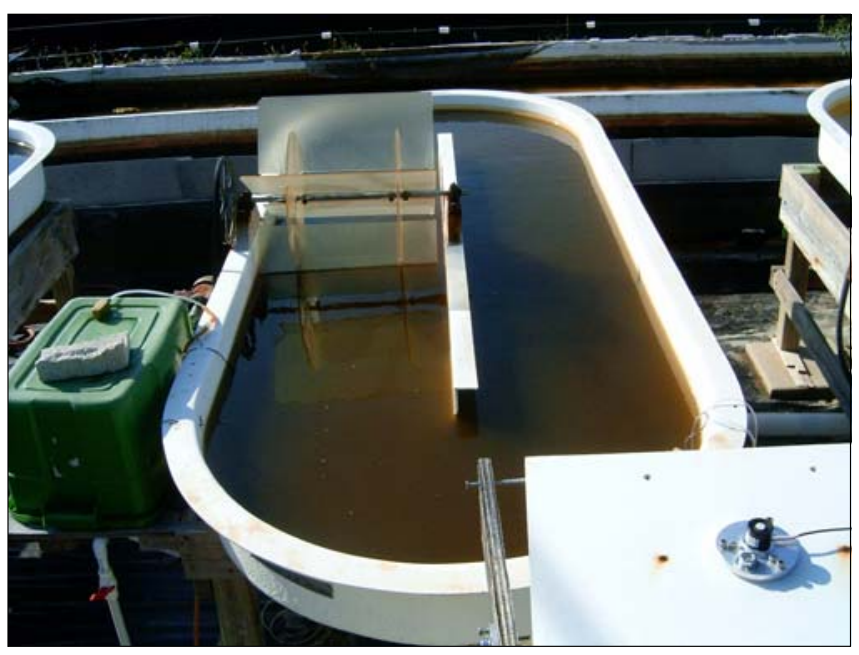

Trial 4. D2-Run 117 (0.5g.m-2 N)

N-Limiting Pond

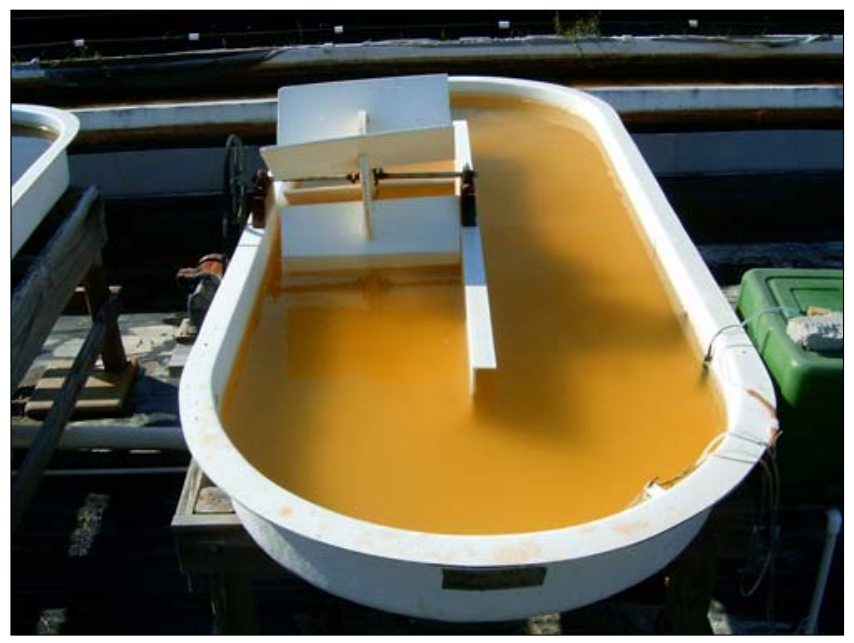

Trial 4. D3-Run 118 (0.09g.m-2 N)

N-Limiting Pond 


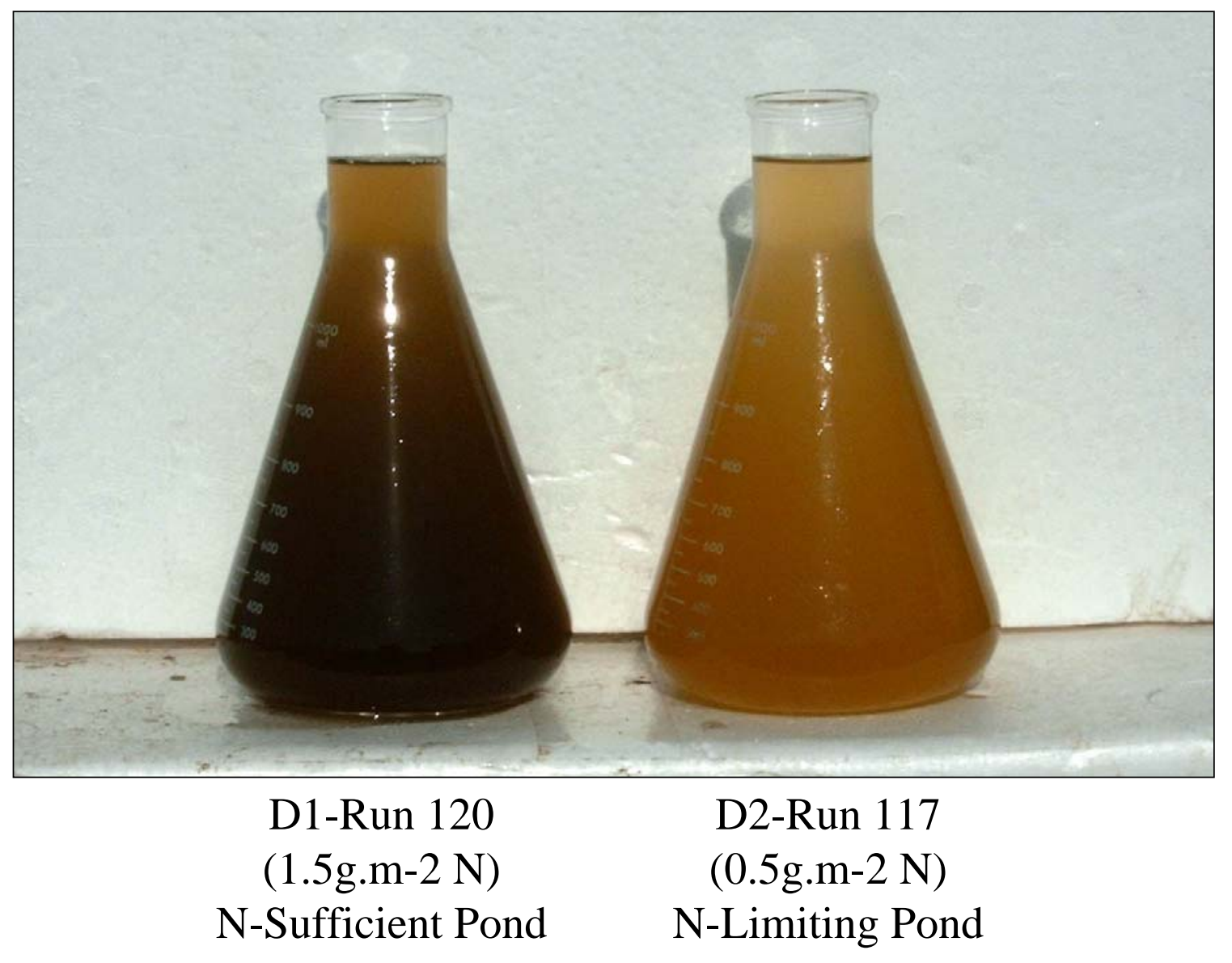


Table 33.

\begin{tabular}{|c|c|c|c|c|c|c|c|c|}
\hline Run \# & $\begin{array}{l}\text { Nitrogen } \\
\text { Feeding } \\
\text { Level } \\
\text { (g.m-2) }\end{array}$ & $\begin{array}{c}\text { Avg } \\
\text { AFDM } \\
\text { (mg.L-1) }\end{array}$ & StDev & $\begin{array}{c}\text { Max } \\
\text { Biomass } \\
\text { Prod. } \\
\text { (g.m-2.d-1) }\end{array}$ & $\begin{array}{c}\text { Avg } \\
\text { Biomass } \\
\text { Prod. } \\
\text { (g.m-2.d-1) }\end{array}$ & StDev & $\begin{array}{c}\text { Avg } \\
\text { Photo. } \\
\text { Efficiency } \\
(\%)\end{array}$ & StDev \\
\hline 59 & 3.0 & 187 & \pm 19 & 16.7 & 14.5 & \pm 2.1 & 4.8 & \pm 1.0 \\
\hline 60 & 1.5 & 195 & \pm 30 & 20.0 & 15.2 & \pm 3.2 & 5.0 & \pm 1.2 \\
\hline 61 & 0.5 & 209 & \pm 33 & 24.9 & 16.1 & \pm 3.8 & 5.3 & \pm 0.8 \\
\hline
\end{tabular}

Trial 2: Nitrogen Limiting in Cyclotella sp. (strain Cyclo J-5) in semi-continuous culture, $2.8 \mathrm{~m}-2$ pond. Dates of compairison: 10/23/2004 - 11/7/2004

Avg daily insolation: 4321 w.hr.m-2.d-1

Avg Max Temp: $30.5^{\circ} \mathrm{C}$, Avg Min Temp: $19^{\circ} \mathrm{C}$

\begin{tabular}{|c|c|c|c|c|c|c|c|c|}
\hline Run \# & $\begin{array}{c}\text { Nitrogen } \\
\text { Feeding } \\
\text { Level } \\
\text { (g.m-2) }\end{array}$ & $\begin{array}{c}\text { Avg } \\
\text { AFDM } \\
\text { (mg.L-1) }\end{array}$ & StDev & $\begin{array}{c}\text { Max } \\
\text { Biomass } \\
\text { Prod. } \\
\text { (g.m-2.d-1) }\end{array}$ & $\begin{array}{c}\text { Avg } \\
\text { Biomass } \\
\text { Prod. } \\
\text { (g.m-2.d-1) }\end{array}$ & StDev & $\begin{array}{c}\text { Avg } \\
\text { Photo. } \\
\text { Efficiency } \\
(\%)\end{array}$ & StDev \\
\hline 76 & 1.0 & 257 & \pm 21 & 15.9 & 14.1 & \pm 2.3 & 5.1 & \pm 0.5 \\
\hline 78 & 1.5 & 246 & \pm 19 & 19.5 & 13.1 & \pm 3.1 & 6.0 & \pm 1.7 \\
\hline 77 & 0.5 & 231 & \pm 49 & 19.5 & 13.1 & \pm 3.1 & 6.0 & \pm 1.7 \\
\hline 78 & 1.5 & 210 & \pm 42 & 15.7 & 12.0 & \pm 2.5 & 5.4 & \pm 1.4 \\
\hline
\end{tabular}

Trial 3: Nitrogen Limiting in Cyclotella sp (strain Cyclo J-5) in semi-continuous culture. $2.8 \mathrm{~m}-2$ pond, Dates of comparison 11/21/2004 - 11/23/2004 (Runs76 \& 78) and 11/16/2004 - 11/28/2004 (Runs 77 \& 78) Avg. daily Insolaton 3967 w.hr.m-2.d-1 (Runs 76 \& 78) and 3229 w.hr.m-2.d-1 (Runs 77 \& 78),

Avg. max water temp $28^{\circ} \mathrm{C}$ (Runs $76 \& 78$ ) and 26 (Runs $77 \& 78$ ). Avg min water Temp. $18^{\circ} \mathrm{C}$ (Runs $76 \& 78$ ) and 17 (Runs $77 \& 78$ ).

\begin{tabular}{|c|c|c|c|c|c|c|c|c|}
\hline Run \# & $\begin{array}{l}\text { Nitrogen } \\
\text { Feeding } \\
\text { Level } \\
\text { (g.m-2) }\end{array}$ & $\begin{array}{c}\text { Avg } \\
\text { AFDM } \\
\text { (mg.L-1) }\end{array}$ & StDev & $\begin{array}{c}\text { Max } \\
\text { Biomass } \\
\text { Prod. } \\
\text { (g.m-2.d-1) }\end{array}$ & $\begin{array}{c}\text { Avg } \\
\text { Biomass } \\
\text { Prod. } \\
\text { (g.m-2.d-1) }\end{array}$ & StDev & $\begin{array}{c}\text { Avg } \\
\text { Photo. } \\
\text { Efficiency } \\
(\%)\end{array}$ & StDev \\
\hline 116 & 1.5 & 194 & \pm 16 & 13.2 & 9.7 & \pm 2.5 & 4.3 & \pm 1.5 \\
\hline 117 & 0.5 & 196 & \pm 13 & 13.1 & 9.3 & \pm 2.6 & 4.2 & \pm 2.1 \\
\hline 120 & 1.5 & 190 & \pm 30 & 12.3 & 8.5 & \pm 2.9 & 2.5 & \pm 1.2 \\
\hline 117 & 0.5 & \#REF! & \pm 27 & 10.5 & 8.1 & \pm 2.6 & 2.4 & \pm 1.0 \\
\hline
\end{tabular}

Trial 4: Nitrogen Limiting in Cyclotella sp (strain Cyclo J-5) in semi-continuous culture. $2.8 \mathrm{~m}-2$ pond, Dates of comparison 1/28/2005 -2/4/2005 (Runs116 vs 117) and 2/9/2005 - 2/16/2005 (Runs 120 vs 117) Avg. daily Insolaton 3182 w.hr.m-2.d-1 (Runs 116 vs 117) and w.hr.m-2.d-1 (Runs 120 vs 117),

Avg. max water temp $22^{\circ} \mathrm{C}$ (Runs 116 vs 117) and 23 (Runs 120 vs 117).

Avg min water Temp. $13^{\circ} \mathrm{C}$ (Runs 116 vs 117 ) and 9 (Runs 120 vs 117 ). 
Table 34.

\section{Nitrogen Limited Growth Experiments Cyclotella J5}

\begin{tabular}{|c|c|c|c|c|c|c|}
\hline \multirow{3}{*}{$\begin{array}{c}\begin{array}{c}\text { Dates of } \\
\text { Experiment }\end{array} \\
10 / 23-11 / 7 / 04\end{array}$} & \multicolumn{6}{|c|}{$\begin{array}{l}\text { Nitrogen Sufficiency Status (\% of N-AGP) } \\
\text { Biomass Productivity (g.m-2.d-1) }\end{array}$} \\
\hline & \multicolumn{2}{|c|}{ Sufficient } & \multicolumn{2}{|c|}{ Near Sufficient } & \multicolumn{2}{|c|}{ Nitrogen Limited } \\
\hline & 60 & 14.5 & 90 & 15.2 & 240 & 16.1 \\
\hline $11 / 21 / 11 / 23 / 04$ & 80 & 13.8 & 110 & 14.1 & & \\
\hline $11 / 16 / 11 / 28 / 04$ & 80 & 12.0 & & & 150 & 13.1 \\
\hline $1 / 28-2 / 4 / 05$ & 83 & 9.7 & & & 200 & 9.3 \\
\hline $2 / 9 / 2 / 16 / 05$ & 60 & 8.5 & & & 240 & 8.1 \\
\hline
\end{tabular}

Nitrogen sufficiency defined as N-AGP equal to 10 .

$\mathrm{N}$ in the seaw ater $=1.7 \mathrm{mg}$.L-1 yielded $50 \mathrm{mg} . \mathrm{L}-1$ algal biomass, or Qmin=3\%. 
Table 35.

Statistical Analysis of Nitrogen Limitation Trials for Cyclotella sp. (strain Cyclo J-5) in Semi-continuous Culture

\begin{tabular}{|c|c|c|c|c|}
\hline \multicolumn{5}{|c|}{$\begin{aligned} \text { Confidence Interval } & =95 \% \\
\text { Avg. } & \text { Biom ass }\end{aligned}$} \\
\hline Run \# & $\begin{array}{l}\mathrm{N} \text {-Le vel } \\
(\mathrm{g} \cdot \mathrm{m}-2) \\
\end{array}$ & $\begin{array}{l}\text { Productivity } \\
\text { (g.m-2.d-1) }\end{array}$ & $\begin{array}{c}t-S \text { tat } \\
\text { (one-tail) }\end{array}$ & $\begin{array}{l}\text { t-Critical } \\
\text { (one-tail) }\end{array}$ \\
\hline 59 & 3.0 & 14.5 & \multirow{2}{*}{2.8} & \multirow{2}{*}{1.8} \\
\hline 61 & 0.5 & 16.1 & & \\
\hline 59 & 3.0 & 14.5 & \multirow{2}{*}{1.1} & \multirow{2}{*}{1.8} \\
\hline 60 & 1.5 & 15.2 & & \\
\hline 60 & 1.5 & 15.2 & \multirow{2}{*}{1.2} & \multirow{2}{*}{1.8} \\
\hline 61 & 0.5 & 16.1 & & \\
\hline 78 & 1.5 & 12.1 & \multirow{2}{*}{1.9} & \multirow{2}{*}{1.8} \\
\hline 77 & 0.5 & 13.2 & & \\
\hline 116 & 1.5 & 9.7 & \multirow{2}{*}{0.7} & \multirow{2}{*}{1.9} \\
\hline 117 & 0.5 & 9.3 & & \\
\hline 120 & 1.5 & 8.5 & \multirow{2}{*}{0.7} & \multirow{2}{*}{1.9} \\
\hline 117 & 0.5 & 8.1 & & \\
\hline
\end{tabular}


Figure 15. Nitrogen Limitation in Semi Continuous Culture of Cyclotella J5. Trial 2.
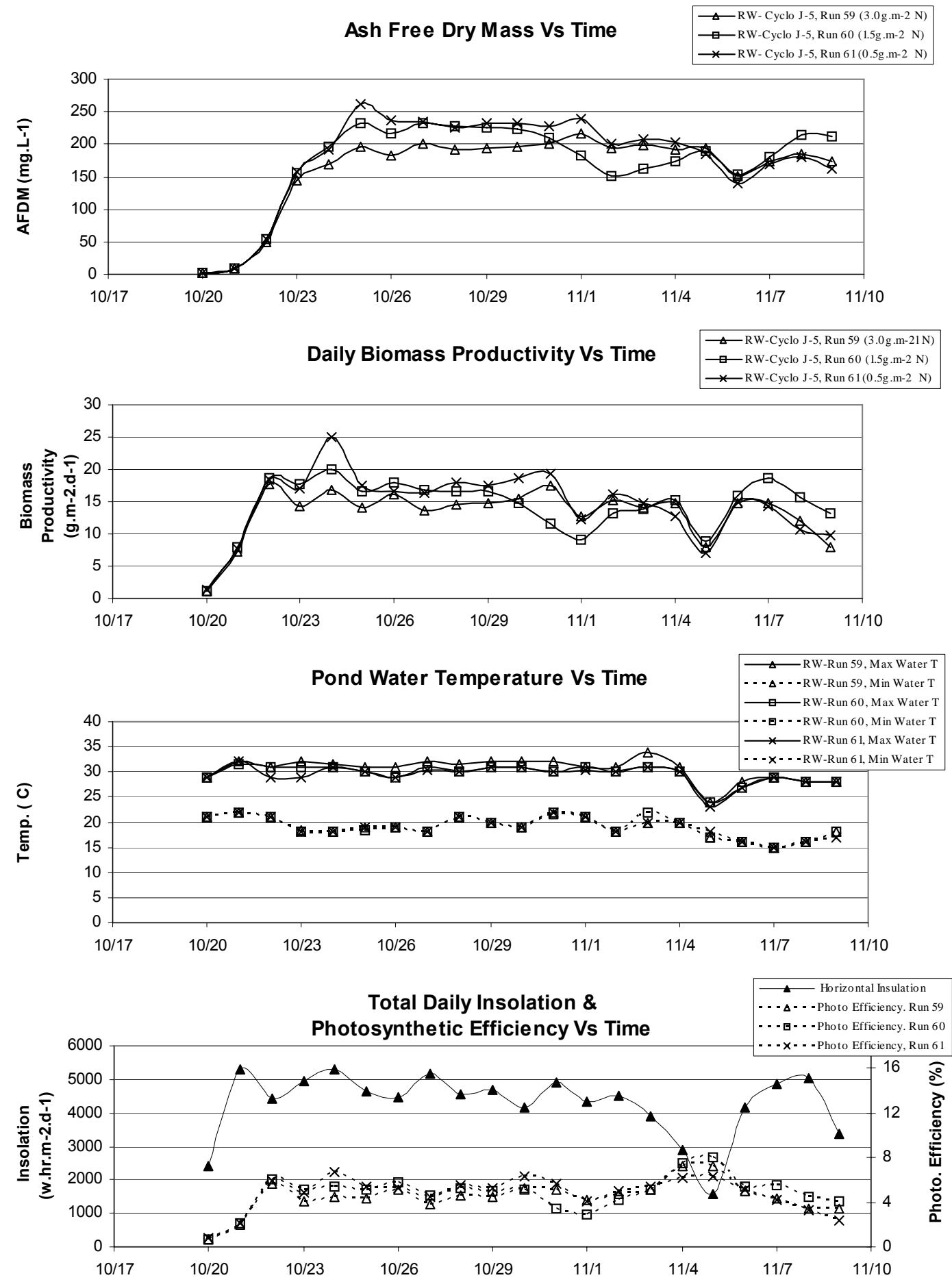
Figure 16 . Nitrogen Limitation in Semi Continuous Culture of Cyclotella J5. Trial 3.

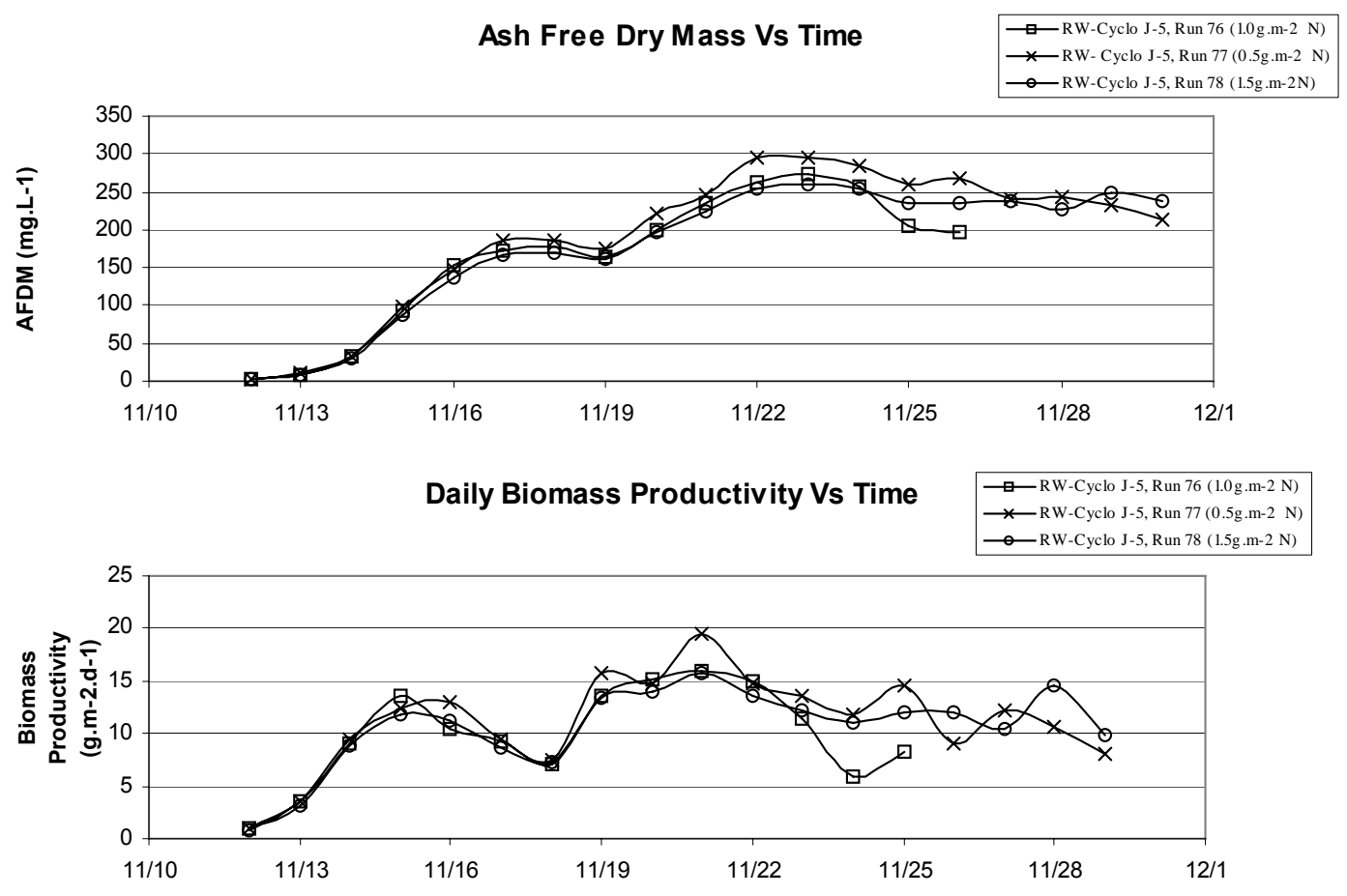

Pond Water Temperature Vs Time

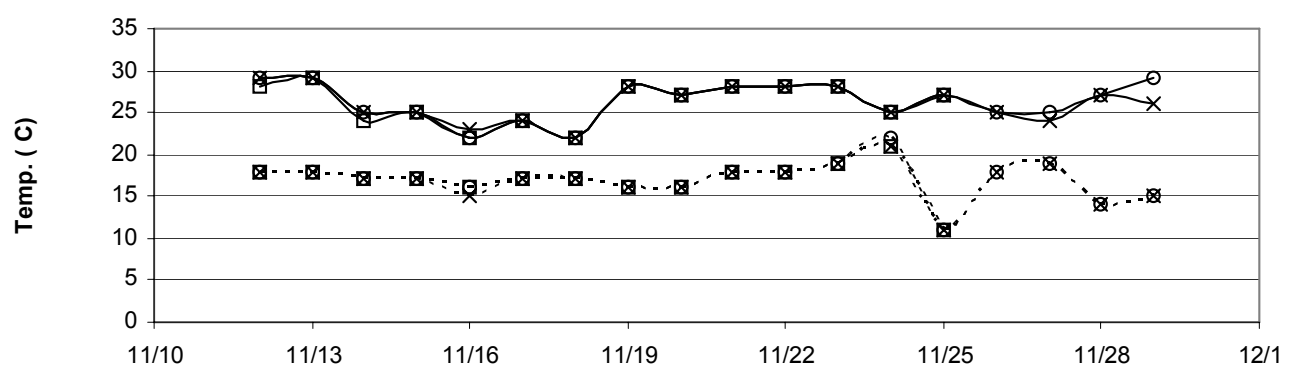

Total Daily Insolation \&

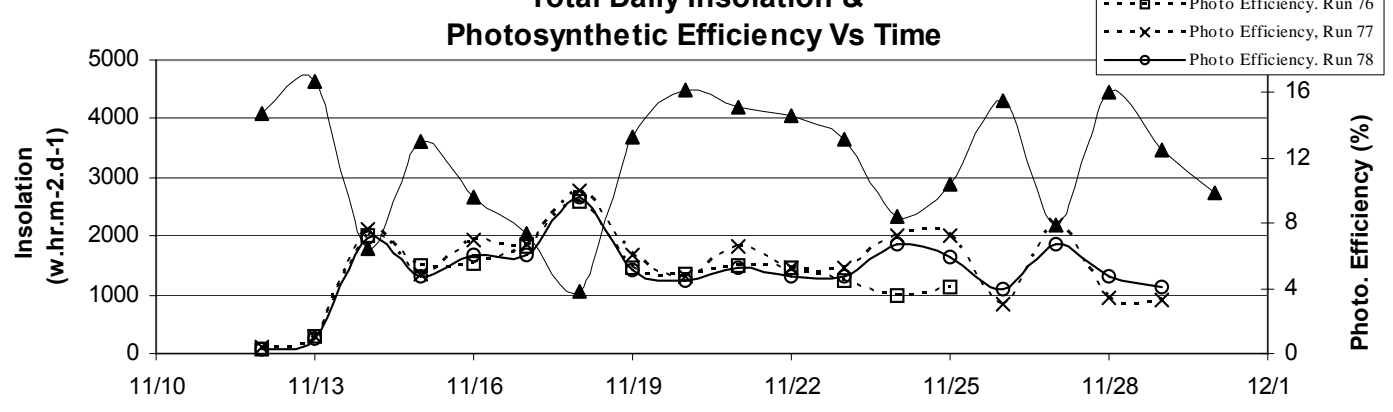




\section{Figure 17. Nitrogen Limitation in Semi Continuous Culture of}

\section{Cyclotella J5. Trial 4.}
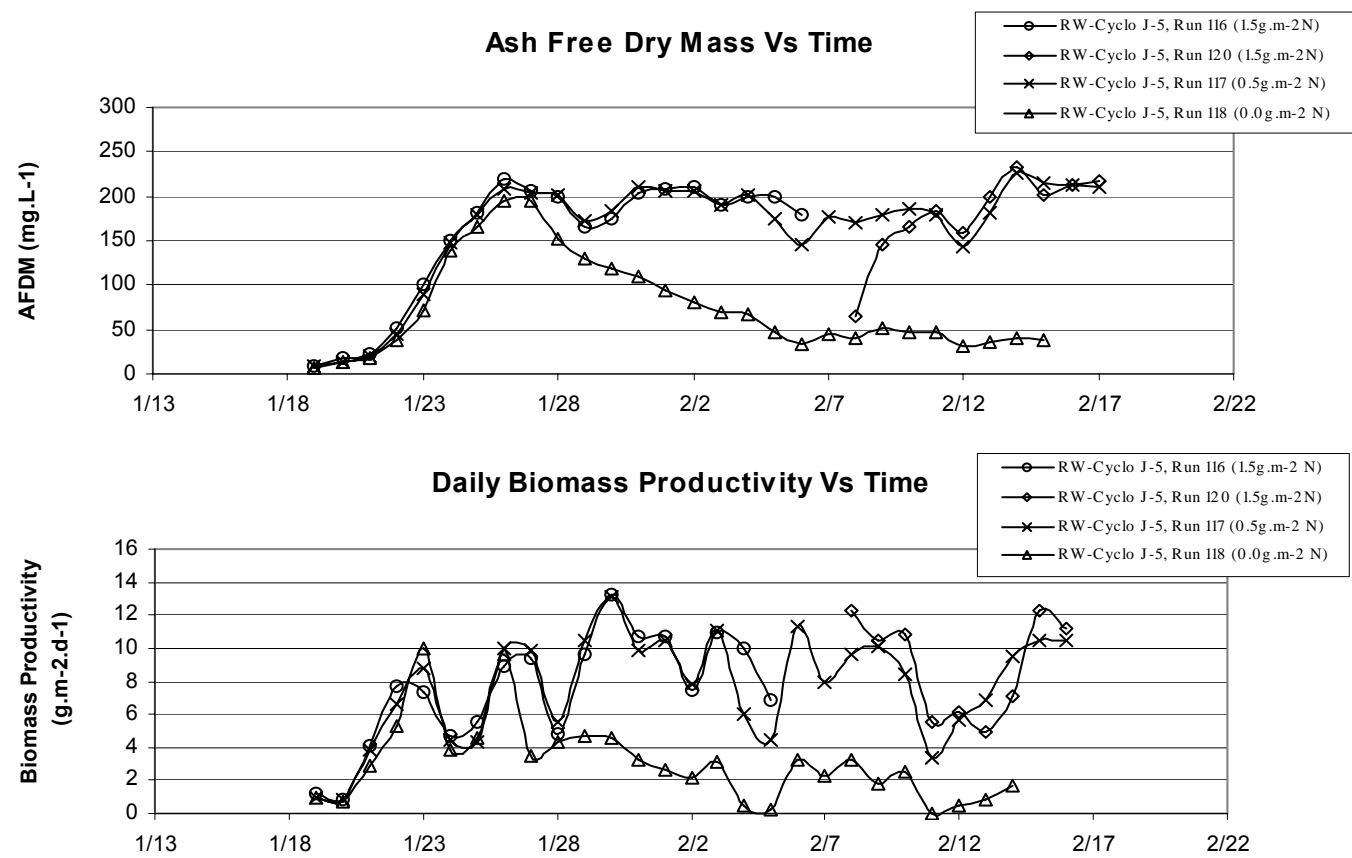

Pond Water Temperature Vs Time

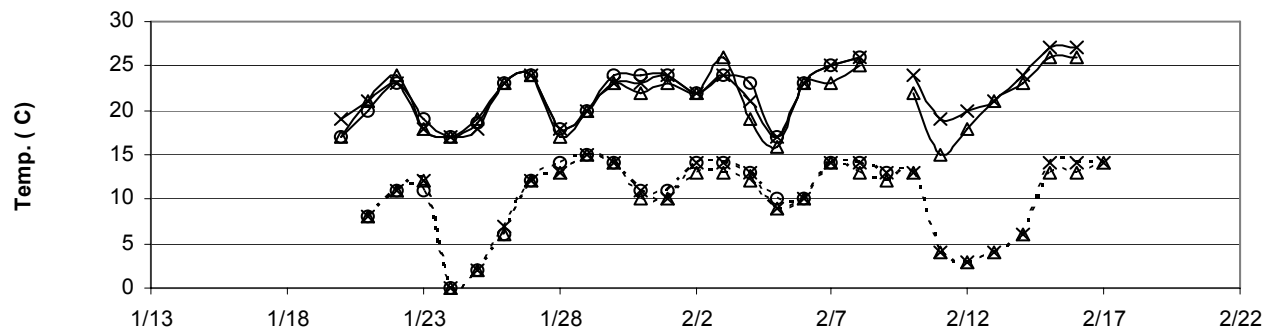

Total Daily Insolation \&

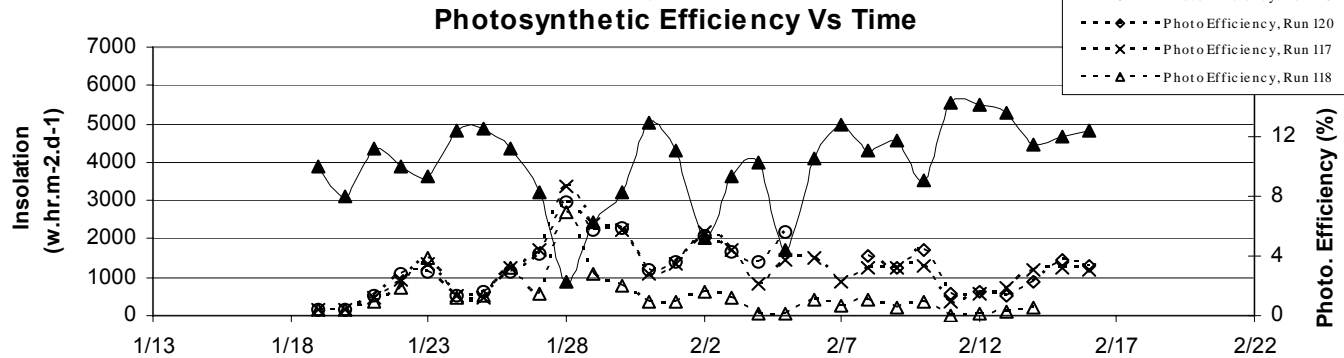


Figure 18 . Nitrogen Limitation in Batch and Semi Continuous Culture of Cyclotella J5.
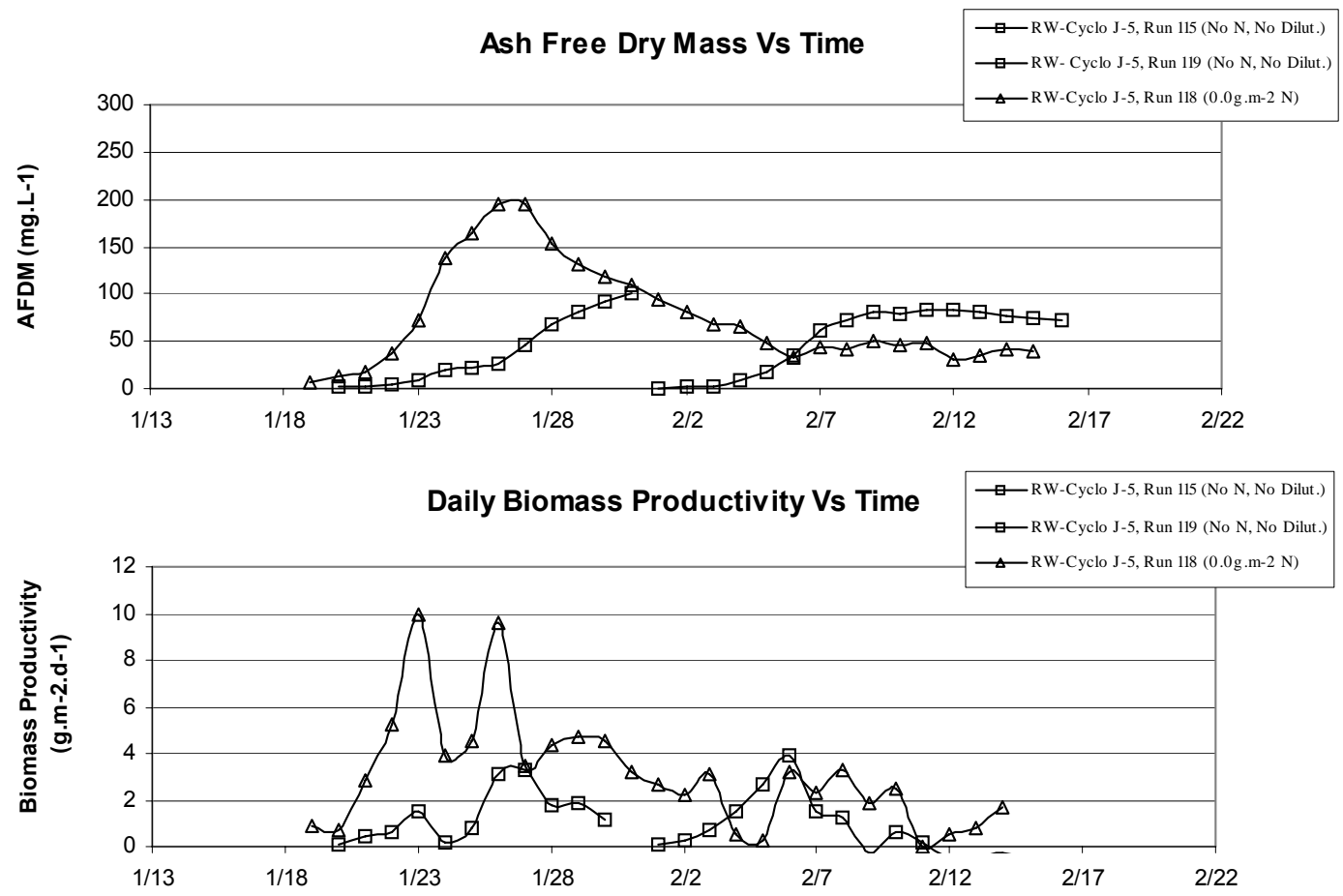

Pond Water Temperature Vs Time
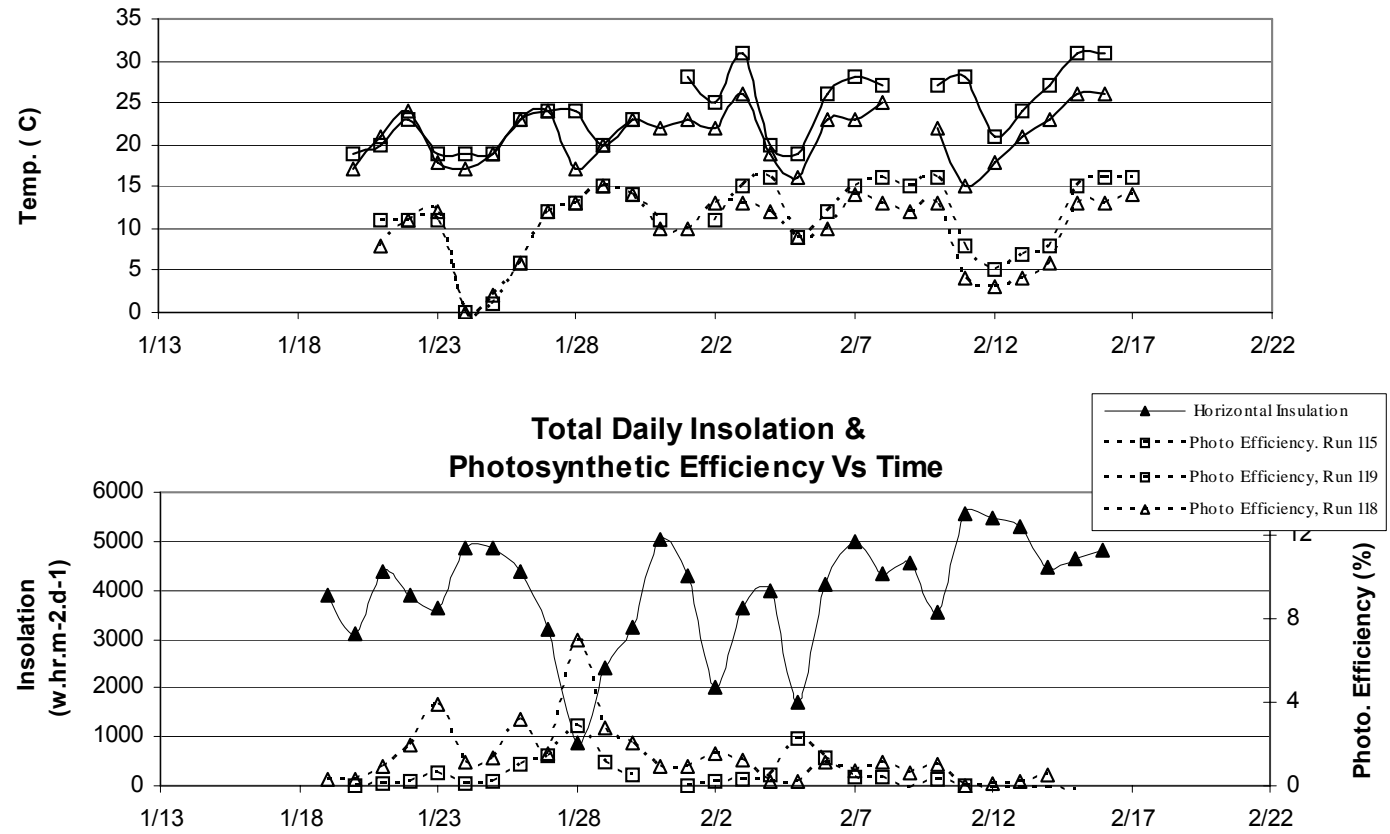


\subsection{Experiments with Nitrogen Limited and Intermittently Covered Ponds}

In the experiments with intermittently covered ponds, it was found that covering $50 \%$ of a pond's surface intermittently only reduced productivity about $20 \%$. It was as if moving the suspension in and out of the light relieved an inhibition that was present when the suspension was continuously exposed to sunlight, bringing the productivity up from the expected $50 \%$ reduction to only the $20 \%$ reduction. When algal suspensions were limited for nitrogen, productivity increased, but only slightly. The pigmentation was greatly reduced in such cultures. If this pigment reduction was due to a decrease in light harvesting pigments, leading to an increase the saturating light intensity, then it would be expected that such cultures would waste fewer photons and exhibit a higher productivity. That this did not happen to any great extent suggested that something else was limiting the full expression of greater productivity. It may be that not only were harvesting pigments reduced, but reaction center numbers were reduced as well. Also with the reduced pigment content, each cell would receive higher light intensities and this may have also triggered a greater down regulation of photosynthesis, which would look like inhibition. If so, then nitrogen limited cultures would be more subject to photo inhibition than nitrogen sufficient ones. To test this hypothesis, four ponds were operated, two with and two without intermittent $50 \%$ coverage. One pond of each pair was nitrogen limited and the other nitrogen sufficient. Four trials of this experiment were performed, in addition to some trials with either N-limited or Nsufficient ponds but not both.

A summary of the results is shown in Table 36. More details of each trial are given in Table 37. The data from the ponds are grouped in two ways: ratios of productivities of $50 \%$ open to $100 \%$ open for $\mathrm{N}$-limited and $\mathrm{N}$-sufficient cases and ratios of productivities of $\mathrm{N}$-limited to $\mathrm{N}$-sufficient for 50 and $100 \%$ open cases. If the N-limited ponds were more photoinhibited by light than the N-sufficient ponds, then the ratio of production of $50 \%$ to $100 \%$ open should be greater for $\mathrm{N}$-limited ponds than for $\mathrm{N}$-sufficient ponds. For the same reason, the ratio of productivities of $\mathrm{N}$-limited to $\mathrm{N}$-sufficient ponds should be greater for $50 \%$ open ponds than for $100 \%$ open ponds. Each of these conditions was true for most trials. Compare the two left hand columns of data in Table 36. The first column shows closer productivity from 50 and $100 \%$ open ponds when the ponds were N-limited. It was more often the case that the differences in production were statistically significant in the Nsufficient cases because covering the ponds had a greater effect than in the $\mathrm{N}$-limited cases. More importantly, when comparing the third and fourth data columns, for the cases in which photo inhibition was relieved by covering $50 \%$ of the pond intermittently, as opposed to not covering the ponds, the $\mathrm{N}$-limited ponds were not only more productive than the $\mathrm{N}$-sufficient ones in all cases, but when compared to the $100 \%$ open ponds the productivity ratios were greater. This implies that for conditions under which photo inhibition is relieved, $\mathrm{N}$-limited cultures out produce N-sufficient ones to a greater extent. Although in two of these cases the productivity ratios look large enough to be statistically significant, they aren't. In each of these cases, one of the ponds had one point farther from the average than the others, creating a higher standard deviation which led to the results not having statistical significance. Without this one point the results would have been statistically different. Clearly, experimental work with outdoor cultures requires considerable experimental efforts. 
Table 36.

\section{Experiments with Nitrogen Limited and Intermittently Covered Ponds in 2005. Cyclotella J5.}

\begin{tabular}{|c|c|c|c|c|c|c|}
\hline \multirow{2}{*}{$\begin{array}{l}\text { Dates of } \\
\text { Expt. }\end{array}$} & \multirow{2}{*}{$\begin{array}{c}\text { N-Limited } \\
\begin{array}{c}50 \% / 100 \% \\
\text { open }\end{array}\end{array}$} & \multirow{2}{*}{$\begin{array}{c}\text { N-Sufficient } \\
\begin{array}{c}50 \% / 100 \% \\
\text { open }\end{array}\end{array}$} & \multirow{2}{*}{$\begin{array}{c}\text { 50\% Open } \\
\mathrm{N} \text {-lim/N-suff }\end{array}$} & \multirow{2}{*}{$\begin{array}{c}\text { 100\% Open } \\
\text { N-lim/N-suff }\end{array}$} & \multicolumn{2}{|c|}{ Degree of N Lim. } \\
\hline & & & & & $\begin{array}{l}50 \% \\
\text { Open }\end{array}$ & $\begin{array}{l}100 \% \\
\text { Open }\end{array}$ \\
\hline $3 / 2-6$ & 0.85---S & & & & $\begin{array}{c}200- \\
225\end{array}$ & 225 \\
\hline $3 / 15-22$ & 0.92---NS & & & & 225 & 275 \\
\hline $3 / 31-4 / 5$ & & 0.79---S & & & 100 & 120 \\
\hline 4/13-18 & 1.00---NS & 0.87---S & 1.07---S & 0.93---S & $\begin{array}{c}150- \\
200\end{array}$ & $\begin{array}{l}200- \\
225\end{array}$ \\
\hline $5 / 10-18$ & 0.92---NS & 0.86---S & 1.16---S & 1.08---NS & 220 & 240 \\
\hline $5 / 27-30$ & 0.91---NS & 0.91---NS & 1.27---NS! & $1.27---S$ & $\begin{array}{c}120- \\
180\end{array}$ & $\begin{array}{c}130- \\
190\end{array}$ \\
\hline $6 / 17-24$ & 0.87---S & 0.86---S & 1.11---NS & 0.98---NS & 220 & 240 \\
\hline $7 / 20-26$ & & $0.81---S$ & & & & \\
\hline
\end{tabular}




\section{Table 37. Nitrogen Limitation and Pond Covering}

Trial 1- Nitrogen Limitation

Dates of Averaging.- 3/2 - 3/6

\begin{tabular}{|c|c|c|c|c|c|c|c|c|c|c|c|}
\hline Pond & $\begin{array}{c}\text { Open } \\
\text { Pond } \\
\text { Surface } \\
\text { Area } \\
\text { (m-2) }\end{array}$ & $\begin{array}{l}\text { Run } \\
\text { No. }\end{array}$ & $\begin{array}{c}\text { Avg } \\
\text { AFDM } \\
\text { (mq.L-1) }\end{array}$ & $\begin{array}{c}\text { Max } \\
\text { Biomass } \\
\text { Prod. }\end{array}$ & $\begin{array}{c}\text { Avg } \\
\text { Biomass } \\
\text { Prod. } \\
\text { g.m-2.d-1 }\end{array}$ & StDev & $\begin{array}{c}\text { Avg } \\
\text { Photo. } \\
\text { Efficiency } \\
(\%)\end{array}$ & $\begin{array}{c}\text { Avg } \\
\text { Daily } \\
\text { Insulation }\end{array}$ & $\begin{array}{l}\text { Avg } \\
\text { Max } \\
\text { Water } \\
\text { Temp } \\
\text { (C) }\end{array}$ & $\begin{array}{l}\text { Avg } \\
\text { Min } \\
\text { Water } \\
\text { Temp } \\
\text { (C) }\end{array}$ & \\
\hline D1 & 1.4 & 121 & 103 & 7.8 & 6.6 & \pm 1.1 & 3.2 & 5004 & 19 & 10 & $50 \%$ Open Area \\
\hline D3 & 2.8 & 122 & 114 & 9.5 & 7.8 & \pm 1.6 & 1.9 & 5004 & 20 & 9 & N-Lmt, Open \\
\hline
\end{tabular}

Trial 2- Nitrogen Limitation

Dates of Overall Exp.- (3/7/2005 - 3/24/2005)

Dates of Averaging.- 3/15 - 3/22

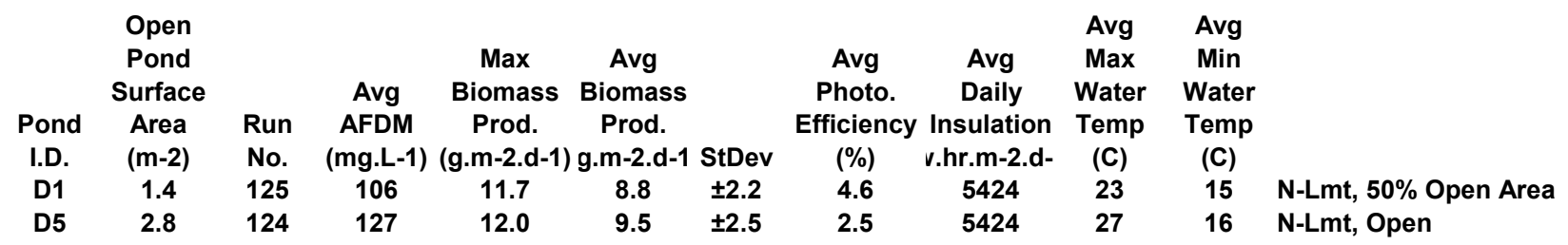

Trial 3- Pond Covering

Per cent of total incident light under slats: 6

Dates of Overall Exp.- (3/25/2005 - 4/6/2005)

Dates of Averaging.- 3/31 - 4/5

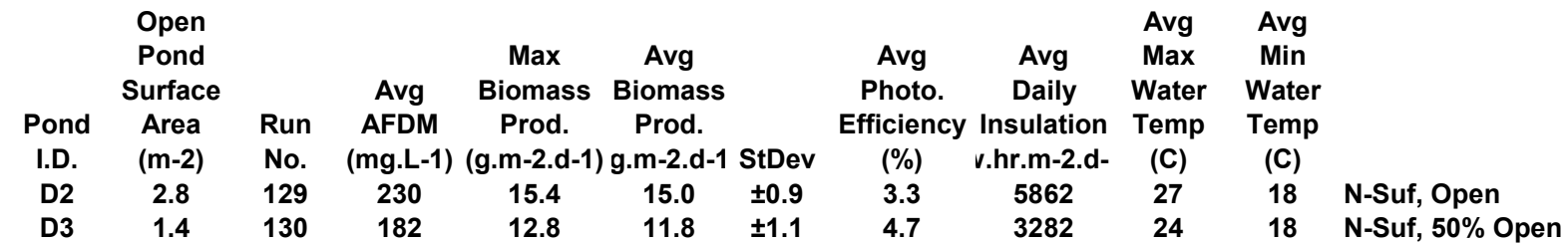

D3, Run 130 . Slatted. $7.5 \mathrm{~cm}$ wide slats, $7.5 \mathrm{~cm}$ wide gap between slats

Trial 4- Nitrogen Limitation and Pond Covering

Per cent of total incident light under slats: 7

Dates of Overall Exp.- (4/08/2005 - 4/20/2005)

Dates of fveraging.- 4/13 - 4/18

\begin{tabular}{|c|c|c|c|c|c|c|c|c|c|c|c|}
\hline $\begin{array}{l}\text { Pond } \\
\text { I.D. }\end{array}$ & $\begin{array}{c}\text { Open } \\
\text { Pond } \\
\text { Surface } \\
\text { Area } \\
(\mathrm{m}-2)\end{array}$ & $\begin{array}{l}\text { Run } \\
\text { No. }\end{array}$ & $\begin{array}{c}\text { Avg } \\
\text { AFDM } \\
\text { (mg.L-1) }\end{array}$ & $\begin{array}{c}\text { Max } \\
\text { Biomass } \\
\text { Prod. } \\
\text { (g.m-2.d-1) }\end{array}$ & $\begin{array}{c}\text { Avg } \\
\text { Biomass } \\
\text { Prod. } \\
\text { g.m-2.d-1 }\end{array}$ & StDev & $\begin{array}{c}\text { Avg } \\
\text { Photo. } \\
\text { Efficiency } \\
(\%)\end{array}$ & $\begin{array}{c}\text { Avg } \\
\text { Daily } \\
\text { Insulation } \\
\text { v.hr.m-2.d- }\end{array}$ & $\begin{array}{l}\text { Avg } \\
\text { Max } \\
\text { Water } \\
\text { Temp } \\
\text { (C) }\end{array}$ & $\begin{array}{l}\text { Avg } \\
\text { Min } \\
\text { Water } \\
\text { Temp } \\
\text { (C) }\end{array}$ & \\
\hline D5 & 2.8 & 131 & 194 & 15.8 & 14.1 & \pm 1.0 & 3.1 & 6965 & 28 & 14 & $100 \%$ Open, N-Lmt \\
\hline D2 & 1.4 & 133 & 169 & 15.6 & 14.1 & \pm 1.4 & 5.3 & 3759 & 24 & 15 & $50 \%$ Open, N-Lmt \\
\hline D1 & 2.8 & 132 & 206 & 17.0 & 15.2 & \pm 1.3 & 3.3 & 6965 & 28 & 14 & $100 \%$ Open, N-Suf \\
\hline D3 & 1.4 & 134 & 180 & 14.1 & 13.2 & \pm 0.6 & 5.0 & 3759 & 24 & 15 & $50 \%$ Open, $N$-Suf \\
\hline
\end{tabular}

D2, Run 133 \& D3, Run 134 . Slatted. $7.5 \mathrm{~cm}$ wide slats, $7.5 \mathrm{~cm}$ wide gap between slats 


\section{Table 37. Continued}

Trial 5- Nitrogen Limitation and Pond Covering

Per cent of total incident light under slats: 5

Dates of Overall Exp.- (5/6/2005 - 5/19/2005)

Dates of Averaging.- 5/10 - 5/18

\begin{tabular}{|c|c|c|c|c|c|c|c|c|c|c|c|}
\hline $\begin{array}{c}\text { Pond } \\
\text { I.D. }\end{array}$ & $\begin{array}{c}\text { Open } \\
\text { Pond } \\
\text { Surface } \\
\text { Area } \\
(\mathrm{m}-2)\end{array}$ & $\begin{array}{l}\text { Run } \\
\text { No. }\end{array}$ & $\begin{array}{c}\text { Avg } \\
\text { AFDM } \\
\text { (mg.L-1) }\end{array}$ & $\begin{array}{c}\text { Max } \\
\text { Biomass } \\
\text { Prod. } \\
\text { (g.m-2.d-1) }\end{array}$ & $\begin{array}{c}\text { Avg } \\
\text { Biomass } \\
\text { Prod. } \\
\text { g.m-2.d-1 }\end{array}$ & StDev & $\begin{array}{c}\text { Avg } \\
\text { Photo. } \\
\text { Efficiency } \\
(\%)\end{array}$ & $\begin{array}{c}\text { Avg } \\
\text { Daily } \\
\text { Insulation } \\
\text { v.hr.m-2.d- }\end{array}$ & $\begin{array}{c}\text { Avg } \\
\text { Max } \\
\text { Water } \\
\text { Temp } \\
\text { (C) }\end{array}$ & $\begin{array}{c}\text { Avg } \\
\text { Min } \\
\text { Water } \\
\text { Temp } \\
\text { (C) }\end{array}$ & \\
\hline D5 & 2.8 & 139 & 269 & 21.8 & 18.4 & \pm 2.7 & 3.7 & 7047 & 31 & 19 & $100 \%$ Open, N-Lmt \\
\hline D2 & 1.4 & 141 & 241 & 19.2 & 17.0 & \pm 1.6 & 6.2 & 3923 & 28 & 19 & $50 \%$ Open, N-Lmt \\
\hline D1 & 2.8 & 140 & 242 & 21.4 & 17.1 & \pm 2.6 & 3.4 & 7047 & 30 & 19 & $100 \%$ Open, N-Suf \\
\hline D3 & 1.4 & 142 & 209 & 19.1 & 14.7 & \pm 2.6 & 5.3 & 3923 & 28 & 19 & $50 \%$ Open, N-Suf \\
\hline
\end{tabular}

D2, Run 141 \& D3, Run 142 . Slatted. $7.5 \mathrm{~cm}$ wide slats, $7.5 \mathrm{~cm}$ wide gap between slats

Trial 6- Nitrogen Limitation and Pond Covering

Per cent of total incident light under slats: 7

Dates of Overall Exp.- (5/23/2005 - 5/31/2005)

Dates of Averaging.- 5/27 - 5/30

\begin{tabular}{|c|c|c|c|c|c|c|c|c|c|c|c|}
\hline $\begin{array}{l}\text { Pond } \\
\text { I.D. }\end{array}$ & $\begin{array}{c}\text { Open } \\
\text { Pond } \\
\text { Surface } \\
\text { Area } \\
(\mathrm{m}-2)\end{array}$ & $\begin{array}{l}\text { Run } \\
\text { No. }\end{array}$ & $\begin{array}{c}\text { Avg } \\
\text { AFDM } \\
\text { (mg.L-1) }\end{array}$ & $\begin{array}{c}\text { Max } \\
\text { Biomass } \\
\text { Prod. } \\
\text { (g.m-2.d-1) }\end{array}$ & $\begin{array}{c}\text { Avg } \\
\text { Biomass } \\
\text { Prod. } \\
\text { g.m-2.d-1 }\end{array}$ & StDev & $\begin{array}{c}\text { Avg } \\
\text { Photo. } \\
\text { Efficiency } \\
(\%)\end{array}$ & $\begin{array}{c}\text { Avg } \\
\text { Daily } \\
\text { Insulation } \\
\text { v.hr.m-2.d- }\end{array}$ & $\begin{array}{l}\text { Avg } \\
\text { Max } \\
\text { Water } \\
\text { Temp } \\
\text { (C) }\end{array}$ & $\begin{array}{l}\text { Avg } \\
\text { Min } \\
\text { Water } \\
\text { Temp } \\
\text { (C) }\end{array}$ & \\
\hline D5 & 2.8 & 143 & 199 & 26.5 & 21.1 & \pm 4.8 & 4.7 & 6438 & 30 & 23 & $100 \%$ Open, N-Lmt \\
\hline D2 & 1.4 & 145 & 180 & 21.7 & 19.2 & \pm 3.0 & 7.4 & 3670 & 30 & 23 & $50 \%$ Open, N-Lmt \\
\hline D1 & 2.8 & 144 & 174 & 17.8 & 16.6 & \pm 1.8 & 3.7 & 6438 & 32 & 23 & $100 \%$ Open, N-Suf \\
\hline D3 & 1.4 & 146 & 149 & 18.4 & 15.1 & \pm 3.0 & 5.9 & 3670 & 30 & 23 & $50 \%$ Open, N-Suf \\
\hline
\end{tabular}

D2, Run $141 \&$ D3, Run 142 . Slatted. $7.5 \mathrm{~cm}$ wide slats, $7.5 \mathrm{~cm}$ wide gap between slats

Cell Quota increased to $1 \%$ for Fe and P for all ponds. 


\section{Table 37. Continued}

Trial 7- Nitrogen Limitation and Pond Covering

Per cent of total incident light under slats: 8

Dates of Overall Exp.- (6/14/2005 - 6/30/2005)

Dates of Averaging.- 6/177 - 6/24

\begin{tabular}{|c|c|c|c|c|c|c|c|c|c|c|c|}
\hline $\begin{array}{l}\text { Pond } \\
\text { I.D. }\end{array}$ & $\begin{array}{c}\text { Open } \\
\text { Pond } \\
\text { Surface } \\
\text { Area } \\
(\mathrm{m}-2)\end{array}$ & $\begin{array}{l}\text { Run } \\
\text { No. }\end{array}$ & $\begin{array}{c}\text { Avg } \\
\text { AFDM } \\
\text { (mg.L-1) }\end{array}$ & $\begin{array}{c}\text { Max } \\
\text { Biomass } \\
\text { Prod. } \\
\text { (g.m-2.d-1) }\end{array}$ & $\begin{array}{l}\text { Avg } \\
\text { Biomass } \\
\text { Prod. } \\
\text { g.m-2.d-1 }\end{array}$ & StDev & $\begin{array}{c}\text { Avg } \\
\text { Photo. } \\
\text { Efficiency } \\
(\%)\end{array}$ & $\begin{array}{c}\text { Avg } \\
\text { Daily } \\
\text { Insulation } \\
\text { v.hr.m-2.d- }\end{array}$ & $\begin{array}{c}\text { Avg } \\
\text { Max } \\
\text { Water } \\
\text { Temp } \\
\text { (C) }\end{array}$ & $\begin{array}{l}\text { Avg } \\
\text { Min } \\
\text { Water } \\
\text { Temp } \\
\text { (C) }\end{array}$ & \\
\hline D5 & 2.8 & 152 & 222 & 20.9 & 17.2 & \pm 3.8 & 5.0 & 4533 & 33 & 23 & $100 \%$ Open, N-Lmt \\
\hline D2 & 1.4 & 154 & 189 & 18.0 & 14.9 & \pm 2.9 & 6.8 & 2908 & 31 & 24 & $50 \%$ Open, N-Lmt \\
\hline D1 & 2.8 & 153 & 214 & 20.9 & 17.5 & \pm 2.3 & 5.1 & 4533 & 33 & 21 & $100 \%$ Open, N-Suf \\
\hline D3 & 1.4 & 155 & 182 & 15.2 & 13.4 & \pm 1.3 & 6.2 & 2908 & 31 & 24 & $50 \%$ Open, N-Suf \\
\hline
\end{tabular}

D2, Run 154 \& D3, Run 155 . Slatted. $7.5 \mathrm{~cm}$ wide slats, $7.5 \mathrm{~cm}$ wide gap between slats

Cell Quota increased to $1 \%$ for Fe and P for all ponds.

Trial 8- Nitrogen Limitation and Pond Covering

Per cent of total incident light under slats: 5

Dates of Overall Exp.- (7/18/2005 - 7/28/2005)

Dates of Averaging.- 7/20 - 7/26

\begin{tabular}{|c|c|c|c|c|c|c|c|c|c|c|c|}
\hline $\begin{array}{l}\text { Pond } \\
\text { I.D. }\end{array}$ & $\begin{array}{c}\text { Open } \\
\text { Pond } \\
\text { Surface } \\
\text { Area } \\
(\mathrm{m}-2)\end{array}$ & $\begin{array}{l}\text { Run } \\
\text { No. }\end{array}$ & $\begin{array}{c}\text { Avg } \\
\text { AFDM } \\
\text { (mg.L-1) }\end{array}$ & $\begin{array}{c}\text { Max } \\
\text { Biomass } \\
\text { Prod. } \\
\text { (g.m-2.d-1) }\end{array}$ & $\begin{array}{c}\text { Avg } \\
\text { Biomass } \\
\text { Prod. } \\
\text { g.m-2.d-1 }\end{array}$ & StDev & $\begin{array}{c}\text { Avg } \\
\text { Photo. } \\
\text { Efficiency } \\
(\%)\end{array}$ & $\begin{array}{c}\text { Avg } \\
\text { Daily } \\
\text { Insulation } \\
\text { v.hr.m-2.d- }\end{array}$ & $\begin{array}{c}\text { Avg } \\
\text { Max } \\
\text { Water } \\
\text { Temp } \\
\text { (C) }\end{array}$ & $\begin{array}{c}\text { Avg } \\
\text { Min } \\
\text { Water } \\
\text { Temp } \\
\text { (C) }\end{array}$ & \\
\hline D5 & 2.8 & 164 & 248 & 22.2 & 20.7 & \pm 1.6 & 4.3 & 6948 & 37 & 26 & $100 \%$ Open, Low pH \\
\hline D1 & 1.4 & 165 & 231 & 23.9 & 19.5 & \pm 3.6 & 4.0 & 6948 & 37 & 26 & $100 \%$ Open \\
\hline D2 & 2.8 & 166 & 182 & 15.5 & 13.8 & \pm 1.4 & 5.1 & 3474 & 35 & 25 & $50 \%$ Open, Sm Gap \\
\hline D3 & 1.4 & 167 & 200 & 16.5 & 15.8 & \pm 0.6 & 6.0 & 3474 & 35 & 25 & $50 \%$ Open, Lg Gap \\
\hline
\end{tabular}

D5, Run 164. Pond ran with lower pH. (7.5)

D2, Run 165 - Slatted. $7.5 \mathrm{~cm}$ wide slats, $7.5 \mathrm{~cm}$ wide gap between slats

D3, Run 166 - Slatted. $14 \mathrm{~cm}$ wide slats, $14 \mathrm{~cm}$ wide gap between slats.

Cell Quota increased to $1 \%$ for Fe and P for all ponds. 


\section{CONCLUSIONS AND TECHNO-ECONOMIC ANALYSIS}

\subsection{Conclusions.}

\subsubsection{Culture Systems - Open Ponds and Closed Photo bioreactors}

The major objective of this project was to develop algal strains and culture techniques that would demonstrate the feasibility of achieving very high microalgae biomass productivities, with a goal of at least 100 metric tons of dry weight organic matter produced per hectare per year at low cost.

To achieve low cost, only open, raceway ponds, mixed by paddle wheel, can be considered, and operations must be based on relatively low mixing velocities $(<30 \mathrm{~cm} / \mathrm{s})$, a $\mathrm{pH}$ range that minimizes $\mathrm{CO} 2$ out gassing (above 8 in seawater systems), and the use of nutrients (including CO2) that are preferably from waste sources (e.g. power plant flue gases) and water sources that are not useful for agriculture or otherwise (e.g. seawater). This project demonstrated the feasibility of using such open ponds, seawater sources and a $\mathrm{pH}$ that minimizes $\mathrm{CO} 2$ out gassing. The use of $\mathrm{CO} 2$ from power plant flue gasses by microalgae is well established, starting with work over 50 years ago (Tamiya, 1953) and with many recent confirming studies (Laws and Berning 1991), including operation of a commercial system in Hawaii (Cyanotech Corp.)

A dominant issue in this field has been the relative productivity of open ponds vs. closed photo bioreactors of various types. An assumption (generally made implicitly but often stated explicitly) of much of the research on using photo bioreactors for algal mass cultures has been that the productivity of open ponds is much, or at least significantly, lower than that of closed photo bioreactors. Of course, one immediate factor is the orientation of the closed photo bioreactors - when vertically oriented the incident sunlight is attenuated and this results in amelioration of the light saturation effect and higher photon utilization efficiency. However, for a higher per unit land area productivity, these vertical photo bioreactors have to be spaced close together, greatly increasing costs (and actually decreasing productivity per unit area of photo bioreactor). Beyond the issue of geometry, other factors are assumed to also increase the productivity of closed photo bioreactors compared to open ponds - with culture density and mixing being the factors most often mentioned.

An important experimental finding from this work was that there are, outside the simple geometry of incident sunlight on a vertical surface (which dramatically changes winter to summer), no other inherent factors that result in an increase in productivity for closed photo bioreactors compared to the open ponds. In other words, when the same intensity of light is incident on both, open ponds are as effective as closed photo bioreactors in solar conversion, at a dramatically lower cost, both capital and operating. Indeed, there is no other choice than open ponds for applications in fuels production or greenhouse gas abatement - as closed photo bioreactors are too expensive for anything but a role, at not more than a $1 \%$ level, in inoculum production for these applications. Similar work, based on research and methods suggested by the P.I., but with different photo bioreactor designs, was recently carried out at 
EniTecnologie (Pedroni $\mathrm{P}$ et al, in preparation), with similar results. Thus the arguments that open ponds cannot be as productive as closed photo bioreactors can now be laid to rest.

\subsubsection{Reduced Pigment Mutants.}

The main experimental objective proposed for this Phase II R\&D effort was to develop reduced antenna size strains and demonstrate the ability to culture these in outdoor ponds with high productivity on a sufficiently sustained basis to allow projection of at least 100 metric tons/hectare-year (mt/ha-yr). Such strains are anticipated, based on strong theoretical arguments and also practical considerations, to achieve such very high productivities.

Towards this objective, Prof.. Juergen Polle, Brooklyn College, who has been working on this problem for some years (Polle et al., 2000, 2001, 2002, 2003) carried out mutagenesis on the Cyclotella strain CYCLOJ used in the outdoor pond experiments here and previously in Phase I (See the Appendix for the report from Prof. Polle). However, the four mutant strains developed and characterized at Brooklyn College by Prof. Juergen Polle (see the Appendix), and tested experimentally in open ponds for productivity under full sunlight intensities, did not achieve the anticipated results - productivities were not higher than for the parent strain. This was a disappointing result.

The reason for this failure was apparent from measuring the second fundamental attribute of these algal strains: in all four cases the mutants grew significantly more slowly under light sufficient conditions than the parent strain. This indicated that additional, growth inhibitory, mutations were present in these strains, additional to the mutations resulting in the reduced pigment content. The lack of increased productivities in these strains can thus be understood as being due to these additional, non-specific, growth inhibiting mutations.

It is clear now that such random mutagenesis is unlikely to yield mutants that are specific enough in the desired phenotype, that is, in a specific in reducing the light harvesting pigments while avoiding any collateral damage to the remainder of their large genetic endowment. This collateral damage shows up as deleterious effects, expressed as reduced maximal growth rates when light is not limiting. The data obtained do not argue against the development of low pigment strains of microalgae for increased productivity, only against the approach of random mutagensis followed by visual screening for low pigment mutations. Therefore, this approach will not be continued and a new approach is now being investigated, under a new project: the genetic engineering of specifically reduced light harvesting pigment strains. This would avoid the problem of non-specific mutations. Unfortunately, no algal strains that can be easily mass cultured in open ponds have a well developed genetic system. Thus, current work is focusing on algal species with well developed genetic systems, even if these are not strains that can be expected to be mass cultured in a practical sense. Their main function will be to demonstrate the feasibility of this approach with real cultures and production processes. 


\subsubsection{Mass Culture Techniques to Increase Photosynthetic Efficiencies.}

This project investigated a number of techniques for mass culture in outdoor ponds that, although some are not practical enough to be directly applied, demonstrate the inherent feasibility of achieving the very high solar conversion efficiencies (and ultimately productivities), on which the main assumption for this project, as well as much other work in this field, are based. A rigorous error analysis was presented, forming the basis for any interpretation of the results presented. Without this, the significance of these results could not the ascertained.

The main technique, already explored during Phase I and more fully during Phase II, for increasing photosynthetic efficiency (though not productivity per unit of pond area) involved light modulation, either by reducing the incident sunlight intensity over the entire pond, or by selectively blocking parts of the pond surface with slats, thereby achieving an intermittent dark-light pattern. As anticipated from the light saturation effect, reducing (by means of a shade cloth) the sunlight intensity impinging on the pond surface decreased productivities, but by much less than based on the reduction in light availability. What was unexpected was that by partially covering the pond with slats, the decrease in productivity was less than half of that anticipated on the basis of the amount light obstructed. This effect is attributed to the overcoming by this means the light inhibition effect, second only to light saturation in reducing algal pond productivities. Such light modulation is only an experimental tool, it cannot be applied in practice to large-scale mass cultures. Previous studies of light modulation in algal ponds, so-called "organized mixing" (Laws et al., 1986), did demonstrate interesting productivity enhancement effects, but also cannot be economically scaled-up.

These experiments indicate the potential of algal mass cultures to achieve increased productivities and photosynthetic efficiencies even when exposed to high light intensities, once the cells are liberated from the evolutionary shackles imposed by the large light harvesting pigment complexes, which are required in nature to allow individual cells to be competitive. Again, this underscores the central objective of this research: to develop algal strains with reduced light harvesting pigments.

Of potentially greater practical applicability in large-scale cultures is the use of nitrogen limitation. In laboratory batch cultures with both green algae and cyanobacteria the P.I. previously observed actually increased productivities when they were moderately $\mathrm{N}$ limited. Although in the open ponds, with the diatom Cyclotella, no major increase in productivity was noted, the fact that productivity did not decline upon nitrogen limitation can be considered a significant finding - it suggests that it will be possible to produce biomass high in carbohydrates or even lipids (oils), without a decrease in productivity. Such biomass would be most suitable for biofuels production - ethanol fermentations for carbohydrates and extraction and conversion to biodiesel for oils (triglycerides).

In conclusion, further work along these lines, in particular nutrient limitation is required. However, fundamentally, the main way to achieve significant increases in productivity is through the development of genetically improved strains. Such strain improvement must be 
carried out alongside the development of mass culture techniques such as explored in the experiments detailed in this report.

\subsection{Techno-Economic Analysis.}

One of the objectives of this project was to carry out a techno-economic analysis of a process for biofuels production, based on the assumption of achieving the projected productivities of 100 mt.ha-1.yr-1. Such economic analyses have been carried out previously by the P.I. and colleagues (Weissman and Goebel, 1987) and were last updated by Benemann and Oswald in 1996. The basic process in all these studies consisted of large paddle earthwork unlined paddle wheel mixed open raceway ponds, supplied with pure or flue gas $\mathrm{CO}$, with the algal biomass high in oils, harvested by bioflocculation-settling (with some provision for flocculant addition) with the harvested biomass converted to biofuels, specifically biodiesel. The major change in the process introduced in the last report was that the final concentration and oil extraction was accomplished with a three phase centrifuge, a major equipment cost.

Figure 38 updates the cost estimates of Benemann and Oswald (1996) to 2006\$, resulting in a total capital cost of about $\$ 75,000$ per hectare. To this must be added an inoculum system as well as a contingency factor, increasing costs by about $20 \%$, to $\$ 90,000 /$ hectare. Similarly, Figure 39 updates the operating costs, amounting to about $\$ 40,000 /$ hectare. To this provisions for both the inoculum production and contingencies must also be added, resulting an estimated $\$ 50,000 /$ hectare, or, for a 100 mtha-1.y-1 productivity, \$500/metric ton of algal biomass.

A major issue is the actual content of the oil in the algal biomass and recovered as biodiesel. In the Benemann and Oswald (1996) analysis, it was assumed that a productivity of 109 mt.ha-1.yr-1 would be achieved (in the base case), with the biomass containing about $40 \%$ oil, and a yield of about 340 barrels of oils per hectare per year. This is rather optimistic, and would require long-term research to achieve. A more plausible mid-term R\&D goal would be a yield of 250 barrels of oil per hectare-year, resulting in a cost of $\$ 200 /$ barrel of raw oil, prior to conversion to biodiesel. Assuming that these productivity goals and cost estimates are reasonable, this is about twice the current price of crude oil, after adding allowances for greenhouse gas abatement and sustainability credits.

The only way to still allow for the process to be considered economically feasible with these projected productivities, biofuel yields and competitive energy prices, is by combing such a process with either wastewater treatment or the co-production of higher value products, such as animal feeds (Benemann 2002, 2003). A detailed analysis is beyond the scope of this report, but it can be readily seen that even a relatively modest value for these co-services or co-products will result in a potentially economically viable process.

Such techno-economic analyses at this point serve mainly to emphasize the need for high productivities in simple, large-scale outdoor systems. Even with by-product and co-product credits, only very high productivities can justify the high capital and operating costs for such processes. Until such high productivities have been demonstrated with outdoor mass 
cultures, efforts to develop specific process components, such as, for example only, oil extraction, are not a high priority. Of course, comparative economic analyses would be useful to refine the R\&D goals that must be achieved. Also a comparison between the production of ethanol, biodiesel and methane from microalgae would be useful.

\subsection{Summary And Future Work}

This project advanced the state-of-the-art of algal mass cultures by demonstrating that such cultures could exhibit very high productivities if the constraints of light inhibition and light saturation could be overcome, and suggested some approaches toward this goal. The practical goal of achieving such high productivities still requires considerable further work, in particular in the development of algal strains with specifically reduced antenna size that overcome the limitations of light saturation but that do not exhibit additional growth reducing mutations. One conclusion from the present work is that such strains will need to be produced with the tools of genetic engineering rather than the simpler mutagenesis/screening protocols used here. That must be the focus of future R\&D in this field. 
Figure 38. CAPITAL COSTS FOR ALGAL BIOFUELS PRODUCTION (in 2006US\$ per hectare of growth pond surface area, total appx. \$75,000/ha) Updated from Benemann and Oswald, 1996, by Craig Jamieson with assistance from John Benemann, 2006

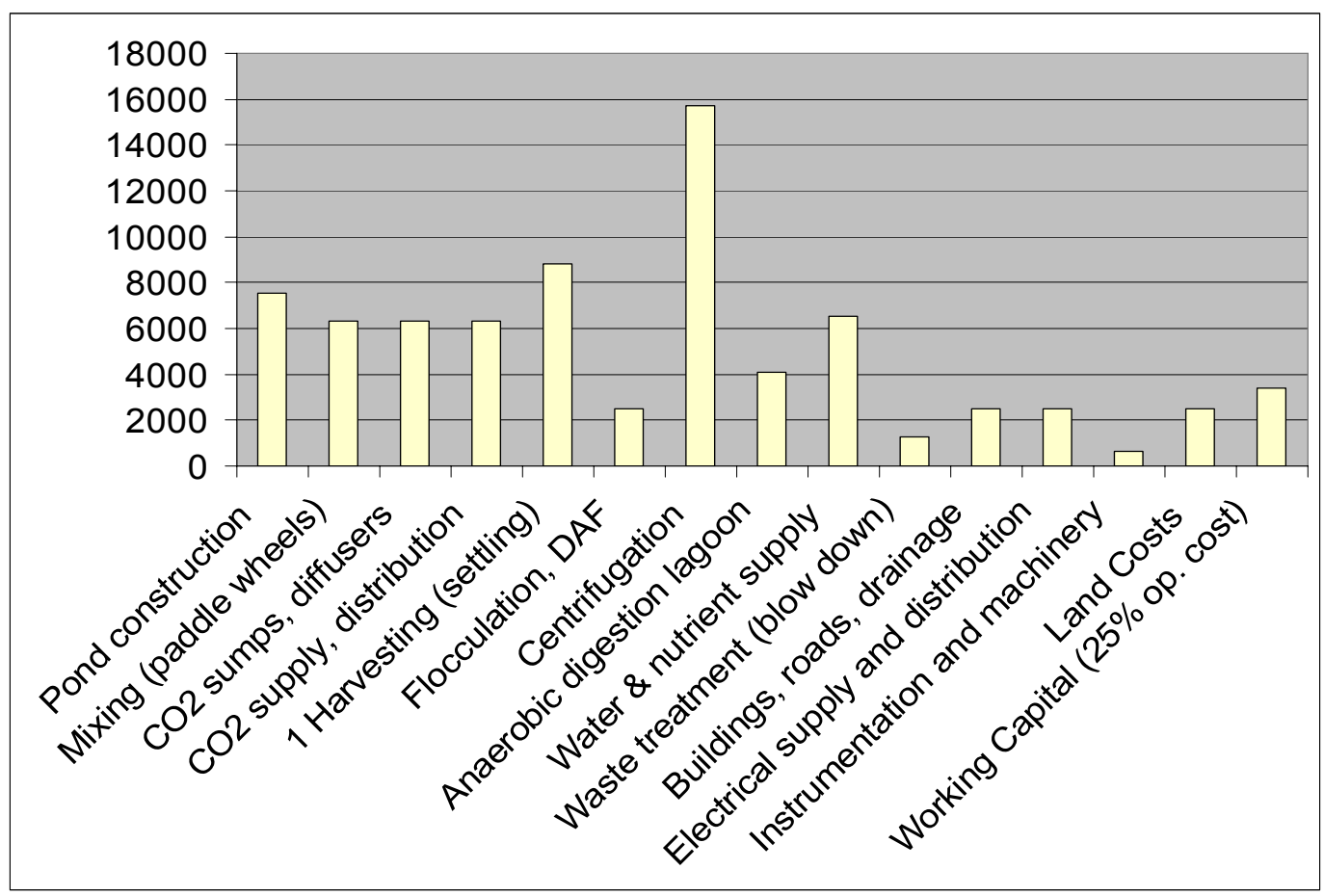

Figure 39. Operating Cost for a Microalgae Biofuels Production Process In \$2006/ha-yr, assume 100 mtha-1yr-1 (total about \$40,000/ha) Updated from Benemann and Oswald, 1996, by Craig Jamieson with assistance from John Benemann, 2006

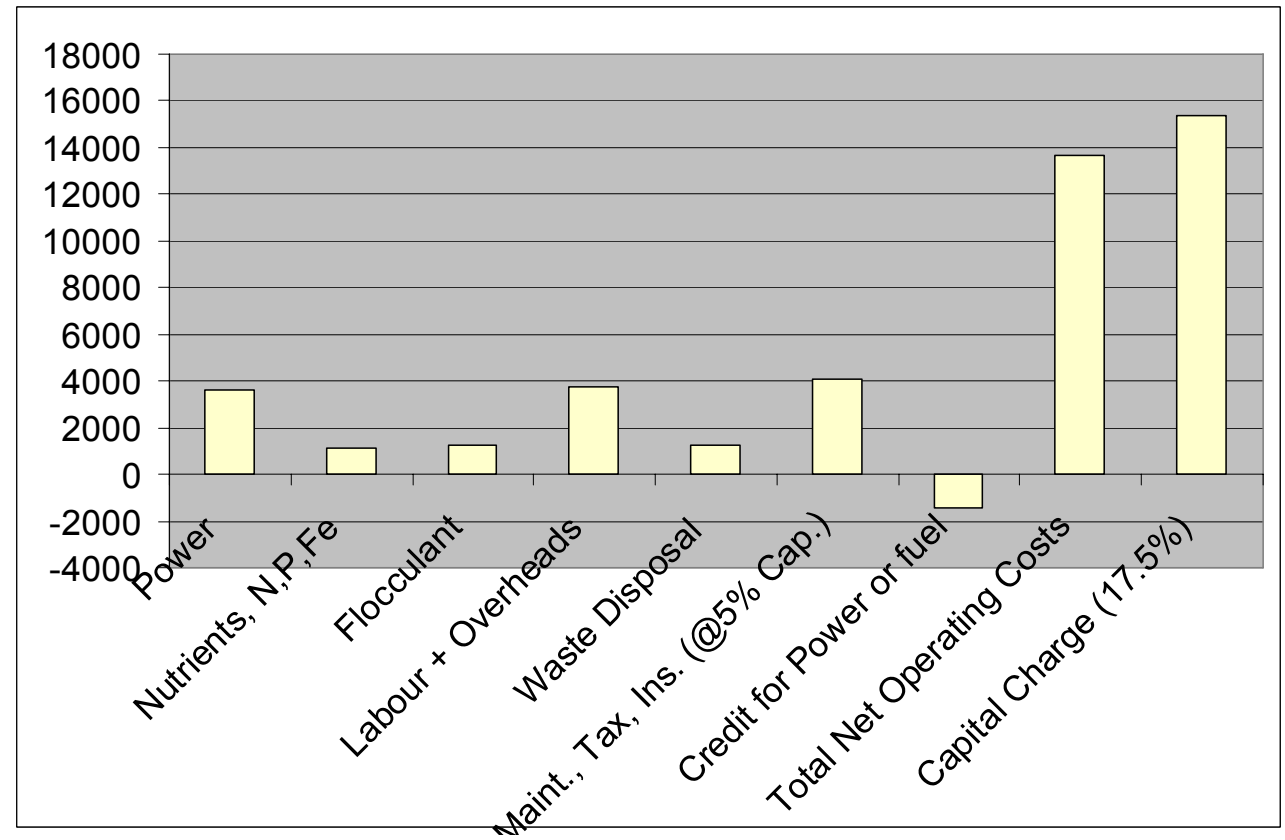




\section{REFERENCES}

Barbosa, MJ, Janssen M, Ham N, Tramper J, Wijffels RH (2003) Microalgae Cultivation in Air-lift Reactors: Modeling Biomass Yield and Growth rate as a Function of Mixing Frequency. Biotech. Bioeng. 82: $170-179$.

Bayless, D.J., GG. Kremer, M.E. Prudich, D.J. Stuart, M.L. Vis-Chiasson, K. Cooksey, and J. Muhs, (May 2001) "Enhanced Practical Photosynthetic $\mathrm{CO}_{2}$ Mitigation". Proceedings of the National Energy Technology Laboratory - Dept. of Energy, First National Conf. Carbon Sequestration.

Benemann JR, Oswald WJ (1996) Systems and Economic Analysis of Microalgae Ponds for Conversion of $\mathrm{CO}_{2}$ to Biomass, Pittsburgh Energy Technology Center, pp.260.

Benemann, J (2003) Technology Roadmap - Biofixation of CO2 and Greenhouse Gas Abatement with Microalgae. Final Report to the U.S. Dept. of Energy, National Energy Technology Laboratory, Morgantown-Pittsburgh, January 14, 2003. (In press)

Benemann JR, Van Olst JC, Massingill MJ, Carlberg JA, Weissman JC, and Brune DE (2002) The Controlled Eutrophication Process: Using Microalgae for $\mathrm{CO}_{2}$ Utilization and Agricultural Fertilizer Recycling. Proceedings of GHGT-6, Kyoto, Japan (in press).

Burlew D (1953) Algae Culture, From Laboratory to Pilot Plant. Carnegie Inst. of Washington, v. 600.

Bush, V cited in Introduction,Burlew D (1953).

Hu Q, Richmond A (1996) Productivity and photosynthetic efficiency of Spirulina platensis as affected by light intensity, algal density and rate of mixing in a flat plate photobioreactor. J. Appl. Phycol. 8: 139 - 145.

Janssen M, Tremper J, Mur LR, Wijffels RH (2002) Enclose Outdoor Photobioreactors Light Regime, Photosynthetic Efficiency, Scale-up, and Future Prospects. Bioeng. Biotech. 81: 193- 210.

Kok B (1953) Experiments in Photosynthesis by Chlorella in Flashing Light. in Burlew JS, ed., Algae Culture, From Laboratory to Pilot Plant. Carnegie Inst. of Washington (Publication 600).

Laws EA, Taguchi S,Hirata J,Pang L (1986) Biotechnol. Bioeng. 28:191.

Laws EA, Berning, JL (1991) A Study of the Energetics and Economics of Microalgal Mass Culture with the Marine Chlorophyte Tetraselmis suecica: Implications for the Use of Power Plant Stack Gases. Biotechnol. Bioeng. 37:936-47. 
Myers J (1957) Algal culture. In Kirk RE, Othmer DF Eds., Encyclopedia Chem. Technol Intersciences. NY 1957, p. 649-680

Nakajima Y, and Ueda R, “The effect of reducing light-harvesting pigment on marine microalgal productivity”. J. App. Phycol., 12: 285 -290 (2000).

Pedroni P, Davison J, Beckert H, Bergman P, and Benemann J (2001) A Proposal to Establish an International Network on Biofixation of $\mathrm{CO}_{2}$ and Greenhouse Gas Abatement with Microalgae". Proceed. Dept. of Energy/NETL 1st National Conf. on C Sequestration. May 2001.

Polle JEW, Benemann JR, Tanaka A, Melis A (2000) Photosynthetic apparatus organization and function in the wild type and a chlorophyll b-less mutant of Chlamydomonas reinhardtii. Planta 211: 335-344

Polle JEW, Niyogi KK and Melis A, (2001) Absence of lutein, violaxanthin and neoxanthin affects the functional chlorophyll antenna size of photosystem-II but not that of photosystemI in the green alga Chlamydomonas reinhardtii. Plant Cell Physiol 42 (5): 482-491

Polle JEW, Kanakagiri S, Jin ES, Masuda T, Melis A (2002) Truncated chlorophyll antenna size of the photosystems- a practical method to improve microalgal productivity and hydrogen production in mass culture. International Journal of Hydrogen Energy :1257-1264

Polle JEW, Kanakagiri S, and Melis A (2003) TLA1, a Chlamydomonas reinhardtii nuclear gene regulates the chlorophyll antenna size of photosynthesis. Planta, in press

Richmond A (1996) Efficient utilization of high irradiance for production of photoautotrophic cell mass: A survey. J. App. Phycol., 8: 381 - 387 (1996).

Sheehan J, Dunahay T, Benemann JR, and Roessler P (1998), "A Look Back at the U.S. Department of Energy's Aquatic Species Program - Biodiesel from Algae". NERL/TP-58024190. National Renewable Energy Laboratory, Golden, CO, 80401, July 1998.

Tamiya (1953) in Burlew, Carnegie Inst of Washington, v 600.

Weissman, J C, (1978) Bioconversion of Solar Energy, PhD Thesis Univ of Calif Berkeley.

Weissman, J.C., R.P. Goebel, and J.R. Benemann, (1987) "Photobioreactor Design: Comparison of Open Ponds and Tubular Reactors”, Bioeng. Biotech., 31: 336-344.

Weissman J C, Goebel RP (1987) Design and Analysis of Pond Systems for the Purpose of Producing Fuels. Solar Energy Research Institute, Golden CO, SERI/STR-231-2840 (1987).

Weissman JC, Tillett DT (1992) Design and Operation of an Outdoor Microalgae Test Facility: Large-Scale System Results", Aquatic Species Project, FY 1989-1990, pp.32-56, NREL, Golden CO., NREL/MP-232-4174 (1992). 


\title{
APPENDIX: FINAL REPORT BROOKLYN COLLEGE SUBCONTRACT
}

\author{
PI: $\quad$ Dr. Jürgen Polle
}

\section{Introduction}

Growth of microalgae becomes saturated in mass cultures already at low light intensities, which are only fractions of the maximum light intensity that the cultures receive during normal days. In consequence, due to low solar conversion efficiencies productivity of microalgae in mass cultures is not optimal.

The goal was to improve the productivity of microalgae in mass culture by increasing the solar conversion efficiencies. The objective is to generate mutants of microalgae that exhibit higher solar conversion efficiencies, because they overcome the low light saturation effect due to a minimal chlorophyll antenna size of the photosystems. Specific aims:

1. Generate microalgae mutants with truncated antenna size of the photosystems.

2. Compare the maximum photosynthetic rate of the wild type to that of the mutants.

\section{Materials and Methods}

\section{Abbreviations used}

AFDW = Ash Free Dry Weight; Chl = chlorophyll; Fv = variable chlorophyll fluorescence emission; Fo = basic chlorophyll fluorescence emission; Fm = maximal chlorophyll fluorescence emission; $\mathrm{L}=$ liter; $\mathrm{mL}=$ milliliter; $\operatorname{Pmax}=$ maximal photosynthetic rate; $\mathrm{R}=$ respiration; $\mathrm{WT}=$ wild type

\section{Algal Culture}

Wild type and mutant strains of Cyclotella spec. were grown in artificial seawater medium. The medium consisted of $299.4 \mathrm{ml} / \mathrm{L}$ distilled water and of $666.6 \mathrm{ml} / \mathrm{L}$ artificial 
seawater (28ppt) made with Instant Ocean ${ }^{\mathrm{TM}}$ seasalts from Aquarium Systems. To each liter medium 2mL Part A and $2 \mathrm{~mL}$ Part B of Kent Micronutrients (F/2) were added to supplement the medium with:

$300 \mathrm{mg}$ Nitrogen / Liter

$40 \mathrm{mg}$ Phosphorus / Liter

$8 \mathrm{mg}$ Iron / Liter

In addition, each liter of medium was supplemented with $25 \mathrm{~mL}$ of Silica solution (40 $\mathrm{mM}, \mathrm{pH}$ 7.5) resulting in a supplement of $28 \mathrm{mg}$ Silica per Liter medium. Each liter of medium contained $5 \mathrm{~mL}$ of Sodium Bicarbonate $(0.5 \mathrm{M}, \mathrm{pH} 7.5)$ resulting in a total amount of $2.1 \mathrm{mg}$ Bicarbonate per Liter medium. The growth medium had a $\mathrm{pH}$ 6.8. It was made fresh and was sterile filtered before starting cultures. To prepare agarose plates the Silica and Sodium Bicarbonate was added to the medium after autoclaving. Stock cultures were kept in $125 \mathrm{~mL}$ Erlenmeyer flasks in artificial seawater medium and were illuminated using daylight fluorescent daylight lamps at about $30 \mu \mathrm{mol}$ photons $\mathrm{m}^{-2}$ $\mathrm{s}^{-1}$.

For measurements algal cultures were grown in $500 \mathrm{~mL}$ Bottles $(3 \mathrm{~cm}$ light path) and supplied with an air/ $\mathrm{CO}_{2}$ gas mixture. The amount of $\mathrm{CO}_{2}$ was regulated by aerating only artificial seawater medium so that it had a $\mathrm{pH}$ of about 7.5. Cultures were illuminated continuously using fluorescent daylight lamps of $\sim 175 \mu \mathrm{mol}$ photons $\mathrm{m}^{-2} \mathrm{~s}^{-1}$ (or at $\sim 70$ $\mu \mathrm{mol}$ photons $\mathrm{m}^{-2} \mathrm{~s}^{-1}$ where indicated).

\section{Generation of Mutants}

Chemical and UV mutagenesis were used to generate mutants of species of the green algae Dunaliella bardawil, Tetraselmis spec. and the Diatom Cyclotella spec. that would have a smaller chlorophyll antenna size of the photosystems than wild type cells. Such mutant strains are characterized by higher saturation light intensities and were expected to have a higher overall photosynthetic rate in outdoor culture.

To this end, initially various salt water/marine algal species and different strains of species were acquired for chemical and UV mutagenesis. The following strains were used in this work:

1. Dunaliella bardawil UTEX 2538 
2. Tetraselmis spec. \#6 (isolated from the Great Salt Lake in Utah by this laboratory)

3. Tetraselmis spec. "E”' (obtained from Dr. M. Huesemann, PNNL)

4. Tetraselmis spec. “OR” (obtained from Dr. M. Huesemann, PNNL)

5. Tetraselmis chuii (obtained from AREAC, Brooklyn College)

6. Cyclotella spec. (obtained from Dr. J. Weissman, SeaAg, Inc.)

\section{Chemical Mutagenesis}

Because every species and strain reacts somewhat different to the chemical used for mutagenesis, first the best growth conditions and procedure for mutagenesis had to be tested and established. For initial chemical mutagenesis, cells of the species Tetraselmis and Cyclotella were grown in liquid culture under continuous light until the mid-log phase. Cells of Tetraselmis were pelleted by 5 min centrifugation at $3000 \mathrm{~g}$. Because of the difference in cell size, cells of Cyclotella were pelleted by 5 min centrifugation at 5,000g. After centrifugation cells were resuspended in $1 \mathrm{ml}$ media. Then EMS was added to the cells. In the beginning different amounts of EMS were used to determine the correct concentration for best results. Based on initial results, later it was decided to use 0.2 M EMS for further rounds of mutagenesis for all algal strains. After addition of EMS, cells were incubated in the dark for $2 \mathrm{hrs}$ and were gently shaken twice. After incubation, cells were centrifuged and washed three times with new media to get rid off excess EMS. After washing cells were plated on agar plates and placed under light $(40 \mu \mathrm{mol}$ photons $\left.\mathrm{m}^{-2} \mathrm{~s}^{-1}\right)$.

\section{$\underline{\text { UV Mutagenesis }}$}

Cells were grown in liquid culture to eliminate clumps during mutagenesis. If the cell density was too high, cells were diluted to prevent shielding. $30 \mathrm{ml}$ liquid culture was used for mutagenesis. The procedure was performed in a dark room to prevent light mediated repair. Cells were kept in a Petri dish and stirred with a stirring bar during illumination. The petri dish containing the cells was placed on a stirrer to keep cells mixing while cells were illuminated with UV. The distance between the plate and the UV lamp was $10 \mathrm{~cm}$. Cells were irradiated with two $15 \mathrm{~W}$ FC15T8 germicidal UV bulbs for 15-20 seconds. After mutagenesis cells were transferred into a flask, covered with aluminum foil and incubated on a shaker at 125 RPM for 16-18 hrs. After dark 
incubation, cells were diluted and plated on agarose plates. The plates were kept under fluorescent daylight lamps at about $30 \mu \mathrm{mol}$ photons $\mathrm{m}^{-2} \mathrm{~s}^{-1}$ for about 10 days to allow cells to grow.

\section{Screening}

After about one week following mutagenesis small colonies appeared on plates that, depending on species and strain, could be screened after an additional one to two weeks. First, cells were screened according to their color. For Tetraselmis pale green colonies were picked which would suggest loss of antenna pigments. For Cyclotella, which exhibits a brownish color due to the carotenoid fucoxanthin as a peripheral antenna pigment, pale brownish or green coloration of colonies would suggest loss of antenna pigments and therefore truncated light-harvesting antenna sizes. Pale brownish and green colonies were picked for further analysis. Initially, the fluorescence imaging apparatus (FluorImager) was not available. Therefore, colonies were transferred to index plates for additional growth and to check for chlorophyll $a / b$ (Tetraselmis) or chlorophyll $a / c$ (Cyclotella) ratios. Tetraselmis mutants with smaller chlorophyll (Chl) antenna size are indicated by higher $\mathrm{Chl} a / b$ ratios. Cyclotella mutants with smaller chlorophyll (Chl) antenna size are indicated by higher $\mathrm{Chl} a / c$ ratios. After the FluorImager was received in October 2003, plates were in a second screen checked for their initial (Fo) and maximal (Fm) Chl fluorescence. Low fluorescence of a colony might indicate smaller Chl antenna size of the photosystem II. Any colonies that had low Chl fluorescence were picked and placed on index plates for further growth. Then in a third screen all potential antenna mutants were checked for their $\mathrm{Chl} a / b$ ratio (Tetraselmis) or Chl $a / c$ ratio (Cyclotella).

\section{Chlorophyll Determination}

Samples of $1 \mathrm{~mL}$ cells were taken and centrifuged in a tabletop Eppendorf centrifuge for $2 \mathrm{~min}$ at maximum speed (14,000 RPM). The supernatant was discarded and the pellet was resuspended in $90 \%$ Acetone. After brief vortexing and storage in the dark at 4 degree Celsius for at least $15 \mathrm{~min}$, the samples were centrifuged at maximum speed for 2 min in a tabletop Eppendorf centrifuge (14,000 RPM). The supernatant was then used to determine the absorption at $630 \mathrm{~nm}, 645 \mathrm{~nm}$ and $664 \mathrm{~nm}$ in a cuvette of $1 \mathrm{~cm}$ pathlength. 
For Cyclotella samples the chlorophyll $a$ and chlorophyll $c$ concentrations were calculated according to Jeffrey and Humphrey (1975):

Chl $a=11.47 * \mathrm{D}_{664}-0.4 * \mathrm{D}_{630}$

Chl $c=-3.73 * \mathrm{D}_{664}+24.36 * \mathrm{D}_{630}$

For Tetraselmis samples the chlorophyll $a$ and chlorophyll $b$ concentrations were calculated in according to Lichtenthaler (1987).

\section{Pigment Extraction}

Pigments of cells were extracted in $90 \%$ Acetone. For extraction of pigments, cells of $1 \mathrm{~mL}$ samples were pelleted by centrifugation at maximum speed for $1 \mathrm{~min}$ in an Eppendorf centrifuge. The pellet was dried by use of a vacuum. Then the cells were resuspended in a 90\% Acetone solution and ruptured by vortexing. After 15 min dark incubation, cell debris was pelleted by centrifugation at maximum speed for $2 \mathrm{~min}$ in an Eppendorf centrifuge. The supernatant was used for experiments.

\section{Cell Counts}

Cells were counted using a Hemacytometer and a Motic AE31 inverted microscope with a magnification of $200 x$.

\section{Dry Weight Determination}

A five-scale balance from Denver Instrument was used. The desiccator was connected to a vacuum. First pans were labled. Then filters and pans were baked at 550C for $15 \mathrm{~min}$. Stored for $5 \mathrm{~min}$ in the desiccator. Pan and filter weights (=pre-weight) were determined before they were stored for about 24 hours in the desiccator. Then about $10 \mathrm{~mL}$ of culture was filtered onto the filter (replicate of 3). Filters were washed with $50 \mathrm{~mL}$ Ammonium Formate. Afterwards pans with filters were baked at 103 Degree $\mathrm{C}$ over night. Then pans and filters with cells were stored in the desiccator for about 1 hour before the weight was determined $\left(=103^{\circ} \mathrm{C}\right.$ weight $)$. Afterwards the pans and filters with cells were baked at $550^{\circ} \mathrm{C}$ for 15 minutes. Then the pans and filters were put back into desiccator for about 1 hour before the weight at $550^{\circ} \mathrm{C}$ was taken $\left(=550^{\circ} \mathrm{C}\right.$ weight $)$. The dry weight was determined according to the following formula: 
Ash free Dry Weight $($ AFDW $)=103^{\circ} \mathrm{C}$ Weight $-550^{\circ} \mathrm{C}$ Weight

\section{Oxygen Measurements}

$2.5 \mathrm{ml}$ of algal culture were used to measure the photosynthesis-light response curve (P-I curve). If necessary, samples were diluted with artificial seawater to yield an $\mathrm{OD}_{570}<$ 0.5. Sample mixing was achieved by use of an integral stirrer driving a magnetic follower. For oxygen evolution measurements the Chlorolab 2 System from Hansatech (see figures below) was used. The LS2 actinic high intensity white light source and fiber optics cables provided illumination. Light intensities were adjusted by a set of neutral density filters. The system was calibrated by aerating artificial seawater $\left(100 \% \mathrm{O}_{2}\right)$ and bubbling with nitrogen $\left(0 \% \mathrm{O}_{2}\right)$. Measurements started by adding $50 \mu \mathrm{L}$ of $0.5 \mathrm{M} \mathrm{NaCO}_{3}$ (12.5 mM total concentration of Bicarbonate in the chamber) before determination of dark respiration, which was followed by stepwise increase of the light intensity. Dark respiration was recorded for 5 minutes and oxygen evolution was recorded for each light intensity for $2 \mathrm{~min}$. The rate of oxygen evolution was calculated using the oxygraph software (Hansatech).
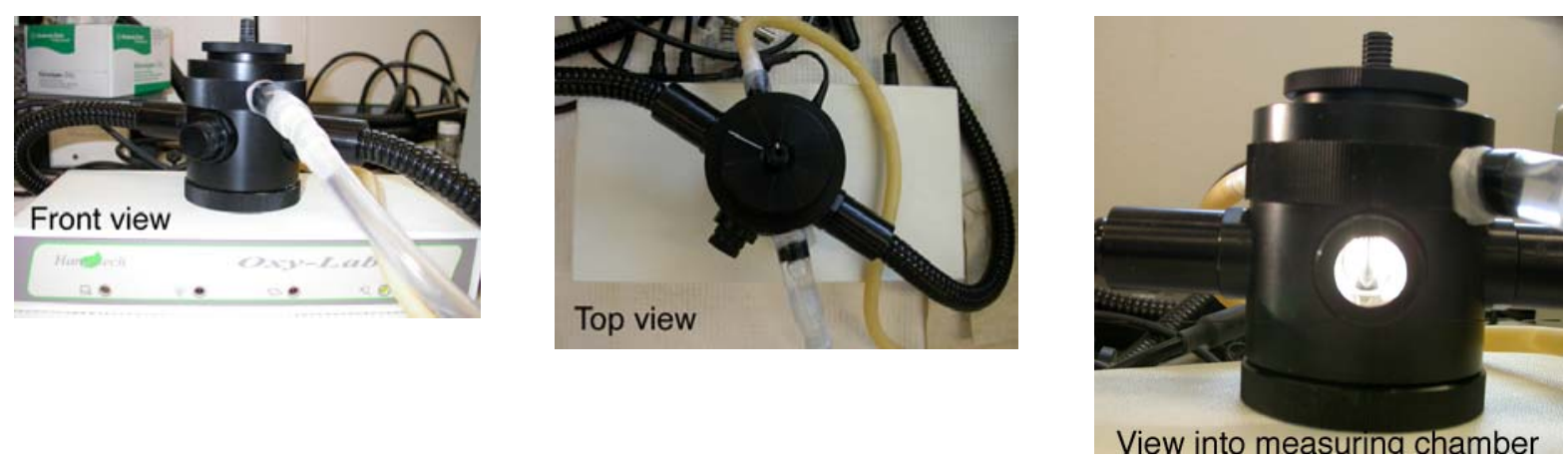

\section{Protein Analysis and Immunoblotting}

$50 \mathrm{~mL}$ of a culture were centrifuged at $6,000 \mathrm{RPM}$ for $5 \mathrm{~min}$. The supernatant was discarded and the pellet containing the cells was frozen at $-70^{\circ} \mathrm{C}$ for $30 \mathrm{~min}$. The frozen sample was then ground up in a mortar in the presence of solubilization buffer that contained $250 \mathrm{mM}$ Tris (pH 6.8), 20\% Glycerol, 7\% SDS, 2 M Urea, and a few $\mu 1$ of Sigma Protease inhibitor Cocktail. The intactness of cells during this treatment was determined by microscopy. After cells were broken, the cell extract was centrifuged in a tabletop Eppendorf centrifuge at maximum speed for 2 min to remove cell debris. Then 
the chlorophyll concentration of the supernatant was determined and the samples were stored at $-70^{\circ} \mathrm{C}$ until further use. Cellular proteins were separated by SDS Gel Electrophoresis using pre-cast 8-16\% Endurance gels from ISC BioExpress. Gels were run in the Mini-Protean 3 cell from BioRad and proteins were transferred onto nitrocellulose. For the immunoblot polyclonal antibodies against the D1 protein of photosystem II and polyclonal antibodies against the psaA/B proteins of photosystem I were used. Proteins were then visualized using chemiluminescence.

\section{Results}

\section{Generation and Screening of Mutants}

After initial test experiments, multiple rounds of mutagenesis were performed with the unicellular green algae Dunaliella bardawil, Tetraselmis spec. and the diatom Cyclotella spec. In each experiment, after mutagenesis cells were spread onto 15 agarose plates. On average, each plate had approximately 100-200 colonies.

For Dunaliella bardawil about 3,000 mutants were generated through chemical mutagenesis. However, no potential antenna size mutants were identified in the initial screening process.

Overall about 26,000 mutants of different Tetraselmis strains were generated and screened for strains with potentially small antenna size. One mutant of Tetraselmis spec. \#6 was found that tested positive in the first two three screening procedures. However, that mutant had only a slightly elevated $\mathrm{Chl} a / \mathrm{Chl} b$ ratio indicating, that the antenna size was not significantly truncated.

For the diatom Cyclotella a total of about 20,000 mutants were generated and screened, and seven mutants with potentially small antenna size were identified based on their coloration and fluorescence imaging. The first mutant $C M-1$ was olive-green in color. The second mutant CM2 was green instead of brown. Because the brown pigment fucoxanthin is a structural component of the light-harvesting proteins in Cyclotella, loss of brown color strongly indicated that the light-harvesting proteins were absent in the mutant CM2. In addition, the spectroscopic analysis indicated that the mutant CM2 lost significant amounts of the accessory pigment $\mathrm{Chl} c$, which is present exclusively in light 
harvesting proteins that make up the outer antenna of photosystems. Taken together, our results suggested that $C M 2$ has only a minimal $\mathrm{Chl}$ antenna size of the photosystems. The mutants $C M 3$ to $C M 7$ all had reduced pigmentation.

In June 2004 the Cyclotella mutant CM1 was used for a repeated round of UV mutagenesis and about 5,000 mutants were generated. Only one potential truncated antenna mutant was identified by its green coloration and low chlorophyll fluorescence emission and named CM1-1.

Figure 1: A) Photograph of an agarose plate with Cyclotella spec. wild type and mutant colonies. B) False color fluorescence image indicating the maximal fluorescence $(\mathrm{Fm})$ for colonies from the plate shown in A). Note that the sensitivity of the FluorImager had to be increased drastically to detect chlorophyll fluorescence emission from the colonies of mutants CM2 and CM1-1.

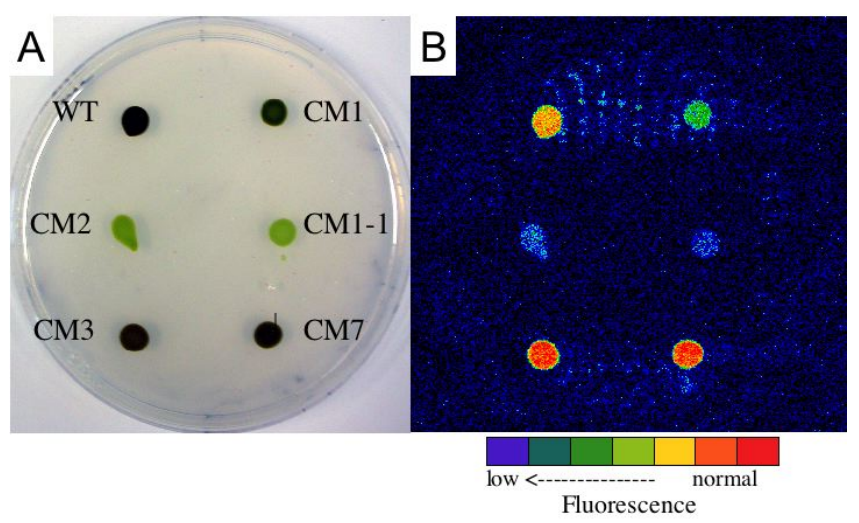

For Cyclotella spec. WT and the mutants CM1, CM1-1, CM2, CM3, and CM7 the fluorescence images (Fo and Fm) were taken to determine the photosynthetic efficiency of Photosystem II. Figure 1 shows that in comparison to the WT, Fm was somewhat decreased for the mutant CM1, but drastically reduced for mutants CM2 and CM1-1. This result strongly indicated that the Chl antenna size of the photosystems was truncated in the mutants CM1, CM2, and CM1-1. In contrast, compared to wild type the mutants CM3 and CM7 had normal levels of Chl fluorescence, suggesting that these mutants had a normal Chl antenna size of the photosystems. To test if at the same time the photosynthetic efficiency of photosystem II was unaffected by changes in the antenna size, $\mathrm{Fv} / \mathrm{Fm}$ ratios were calculated $[\mathrm{Fv} / \mathrm{Fm}=(\mathrm{Fm}-\mathrm{Fo}) / \mathrm{Fm}]$ and compared (Table 1). The slightly reduced Fv/Fm values of CM1, CM1-1, and CM2 indicate that photosystem II in 
those mutants had somewhat reduced photosynthetic efficiency when compared to the wild type. However, the values measured for the mutants CM1, CM1-1, and CM2 are suggesting that the photosynthetic efficiency of photosystem II in these mutants is still almost optimal.

The third screening step ( $\mathrm{Chl} a / \mathrm{Chl} c$ ratio) revealed that the $\mathrm{Chl} a / \mathrm{Chl} c$ ratios of CM1, CM1-1, and CM2 were two to three times higher in the mutants than those of the wild type, suggesting strongly that the $\mathrm{Chl}$ antenna size of the photosystems were drastically reduced in the mutants as compared to the wild type. In contrast, the mutants CM3 and CM7 had normal ratios of $\mathrm{Chl} a / \mathrm{Chl} c$, indicating that they are not antenna mutants. These results were in agreement with the results from the fluorescence imaging screening step.

Table 1. The Fv/Fm value for colonies of Cyclotella spec. wild type and various mutants from agarose plate (Figure 1) is shown. The $\mathrm{Chl} a / c$ ratio is displayed for wild type and mutant cells grown in liquid cultures.

\begin{tabular}{lcc}
\hline Strain & Fv/Fm & Chl $\boldsymbol{a} / \boldsymbol{c}$ \\
\hline Wild type & 0.77 & $\sim 8$ \\
CM1 & 0.73 & $\sim 16$ \\
CM1-1 & 0.74 & $\sim 25$ \\
CM2 & 0.73 & $\sim 21$ \\
CM3 & 0.78 & $\sim 8$ \\
CM7 & 0.79 & $\sim 8$ \\
\hline
\end{tabular}

\section{Analysis of the Cyclotella Mutants CM1, CM2, and CM1-1}

Because the olive-green mutant CM1 and the two green mutants CM1-1 and CM2 passed all three screening steps, these mutants were used for analysis of their photosynthetic performance. For further analysis cells of wild type and the mutants CM1, CM1-1, and CM2 were grown in liquid cultures under simulated mass culture conditions as described above.

Figure 2: Shown are samples of liquid cultures of Cyclotella spec. WT, CM1, CM2, and CM1-1. Note, the different coloration of all three mutants. 


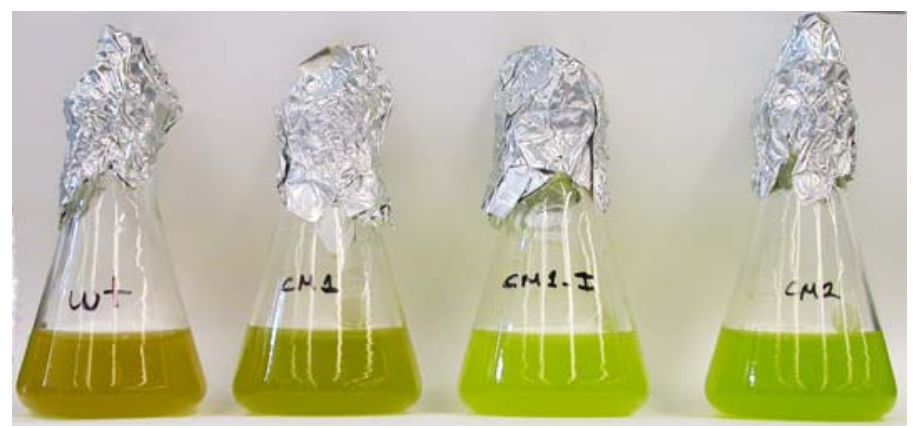

Samples taken from the batch cultures during the light limited growth phase are shown in Figure 2. All mutant had the same coloration as shown during growth on agarose plates. However, it has to be noted that the cultures of CM1-1 and CM2 lost their green coloration and turned brownish late in the light limited growth phase. This result suggested that when severely light limited, the mutant cells altered the pigment composition of their light harvesting complexes (See Figure 12).

Several independent experiments were performed with wild type, CM1, CM1-1, and CM2 grown in batch cultures. The following parameters were determined for wild type and mutants at different times during the batch culture growth phases:

1. \% Chl per AFDW

2. Pmax per mg AFDW

3. Respiration per mg AFDW.

\section{Chlorophyll Content of Cyclotella WT and Mutant Strains}

Figure 3 shows the amount of Chl per biomass (AFDW) for WT, CM1-1, CM1, and CM2 in dependency of the growth phase of batch cultures (plotted as mg AFDW/L). For the wild type, CM1-1, and CM2 the amount of chlorophyll per AFDW (\% Chl/mg AFDW) decreased in dense batch cultures. For the mutant CM1 only values for the light limited growth phase could be obtained. Table 2 compares the Chl content per AFDW in the light limited growth phase for WT and all three mutants.

Table 2: Comparison of the content of $\mathrm{Chl}$ per AFDW in the light limited growth phase of batch cultures (500 mg - 1,000 mg AFDW/L) of WT, CM1, CM1-1, and CM2. The standard deviation is given.

\begin{tabular}{ccccc}
\hline & WT & CM1 & CM2 & CM1-1 \\
\hline \% Chl / AFDW & $0.91 \pm 0.166$ & $1.12 \pm 0.207$ & $0.0 .85 \pm 0.126 .63 \pm 0.088$ \\
\hline
\end{tabular}

Figure 3: Shown are the chlorophyll (Chl) concentrations on an "ash free dry weight" basis for wild type and pigment mutants of the alga Cyclotella. In dependency of the 
growth of the cultures measured as the biomass concentration (AFDW/L) A) $\%$ Chl/AFDW for wild type (WT) and CM1-1. B) \% Chl/AFDW for WT and CM1. \% Chl/AFDW for WT and CM2.
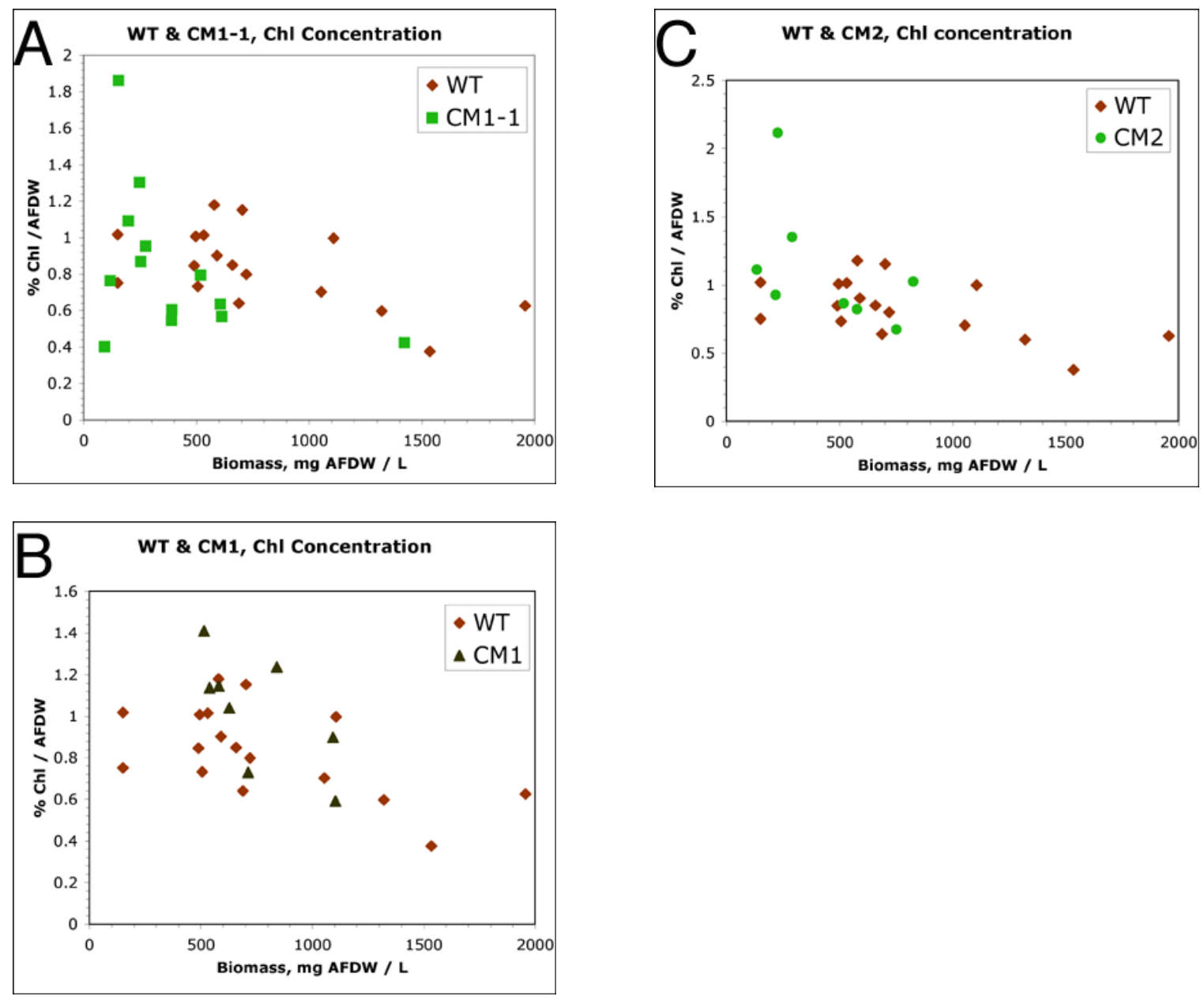

\section{Determination of the Photosynthetic Parameters of Cyclotella WT and Mutants}

Oxygen evolution measurements were performed as described above for the WT and the mutants CM1, CM1-1, and CM2. For measurements, samples were taken from batch cultures at different growth phases. Samples from dense cultures were diluted for measurements to obtain values of less than $\mathrm{OD}_{570}$ of 0.5 . Figure 4 shows the maximal rate of oxygen evolution (Pmax) on a per AFDW basis representing the maximal photosynthetic rate of the algal strains. Figure 4A shows that Pmax is very similar for WT and mutant cultures indicating that their maximal photosynthetic rates are similar. 
Figure 4: Shown are the maximal photosynthetic rates (Pmax) for Cyclotella spec. WT, CM1-1, CM1, and CM2 in dependence of the biomass (mg AFDW/L) in the batch culture.
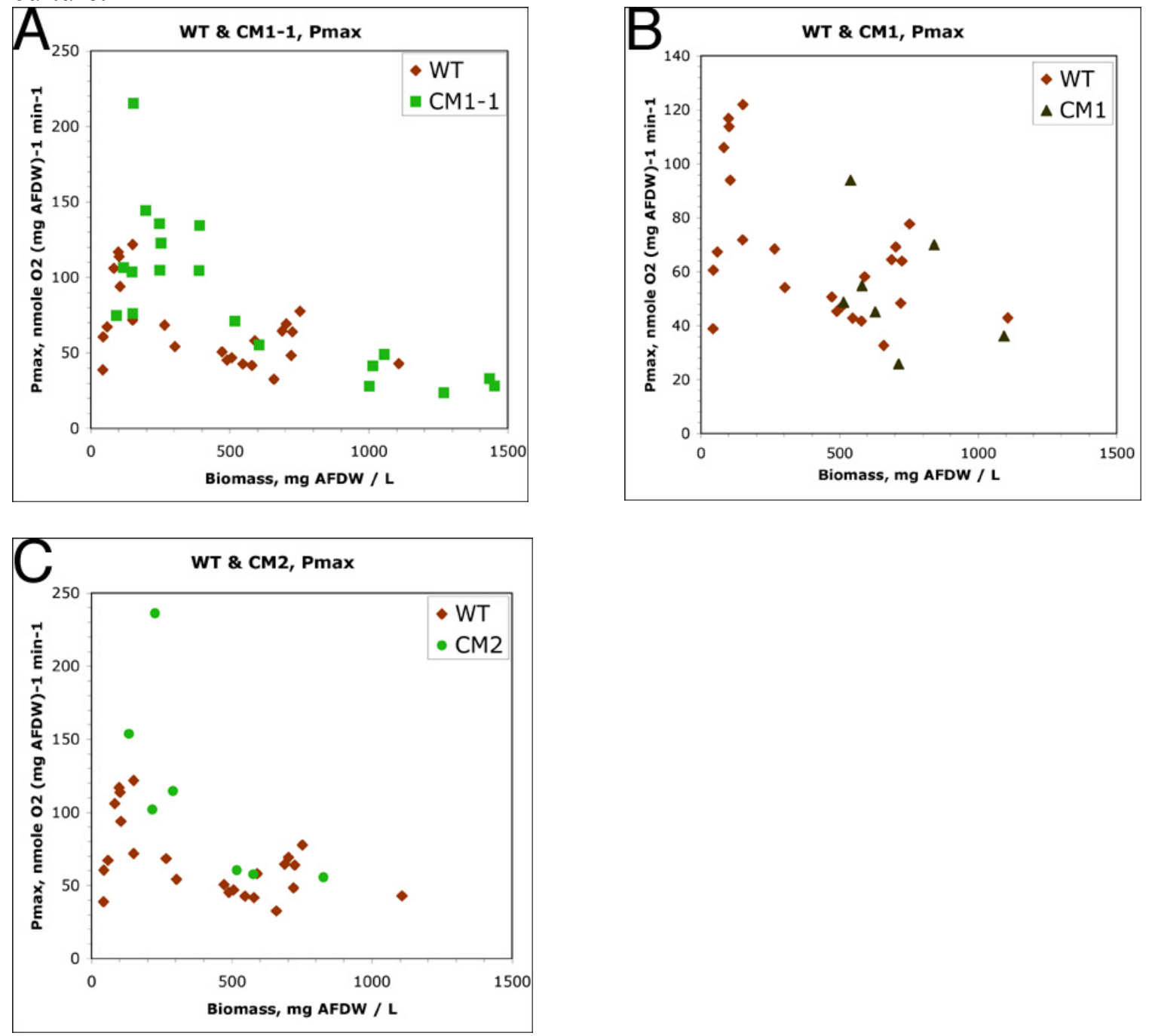

Based on the Pmax determined in the early to mid light-limited growth phase of batch cultures, the specific growth rate was calculated from Pmax according to the following formula:

1 nmole $\mathrm{O}_{2} / \mathrm{mg}$ AFDW min

$$
\begin{aligned}
& =18.53 \mathrm{ng} \text { biomass } / \mathrm{mg} \text { biomass (AFDW) min } \\
& =0.0267 \mathrm{mg} / \mathrm{mg} \mathrm{d} \\
& =0.0267 \mathrm{~d}^{-1}
\end{aligned}
$$

$=>$ Samples taken from batch cultures had maximum photosynthetic rates of $\mathrm{X}$ nmole $\mathrm{O}_{2}$ $(\mathrm{mg} \text { AFDW })^{-1} \mathrm{~min}^{-1}$. This compares to maximal biomass production rates of: $\mathrm{X} * 0.0267 \mathrm{~d}^{-1}$ 
Table 3: Comparison of Pmax and corresponding $\mu$ max in the light limited growth phase of batch cultures (500 mg - 1,000 mg AFDW/L) of WT, CM1, CM1-1, and CM2. The standard deviation is given.

WT

CM1

CM2

CM1-1

\begin{tabular}{lcccc}
\hline $\begin{array}{l}\text { Pmax, } \mathrm{nmole}_{2}(\mathrm{mg} \\
\text { AFDW })^{-1} \mathrm{~min}^{-1}\end{array}$ & $53.7 \pm 13.2$ & $56.3 \pm 21.3$ & $0.58 .0 \pm 1.9$ & $48.8 \pm 16.0$ \\
\hline $\begin{array}{l}\text { Corresponding } \\
\text { maximum biomass } \\
\text { production rate, } \mathrm{d}^{-1}\end{array}$ & 1.54 & 1.5 & 1.55 & 1.3 \\
\hline
\end{tabular}

In summary, at higher biomass concentrations during the light limited growth phase of batch cultures WT and all three mutants had similar maximal photosynthetic rates. Based on the calculation of maximum biomass production rates from Pmax, this indicated that when grown in dense mass cultures, all mutants would have similar growth rates as the wild type, but would not outperform the wild type in biomass accumulation. 


\section{Respiration of Cyclotella WT and mutants}

In addition to the maximal rates of photosynthesis, also the rate of respiration per biomass was determined and is shown for WT and all three mutants in comparative plots in Figure 5. For all mutants the rates of respiration were similar to those of the WT. This result suggested that there exist no mutations in $\mathrm{CM} 1, \mathrm{CM} 1-1$, or $\mathrm{CM} 2$ that affected respiratory processes in the mutants.

Figure 5: Displayed are the respiration rates per biomass for Cyclotella WT, CM1-1, $\mathrm{CM} 1$, and $\mathrm{CM} 2$ in relation to the biomass concentration $(\mathrm{mg}$ AFDW/L) in the batch culture.
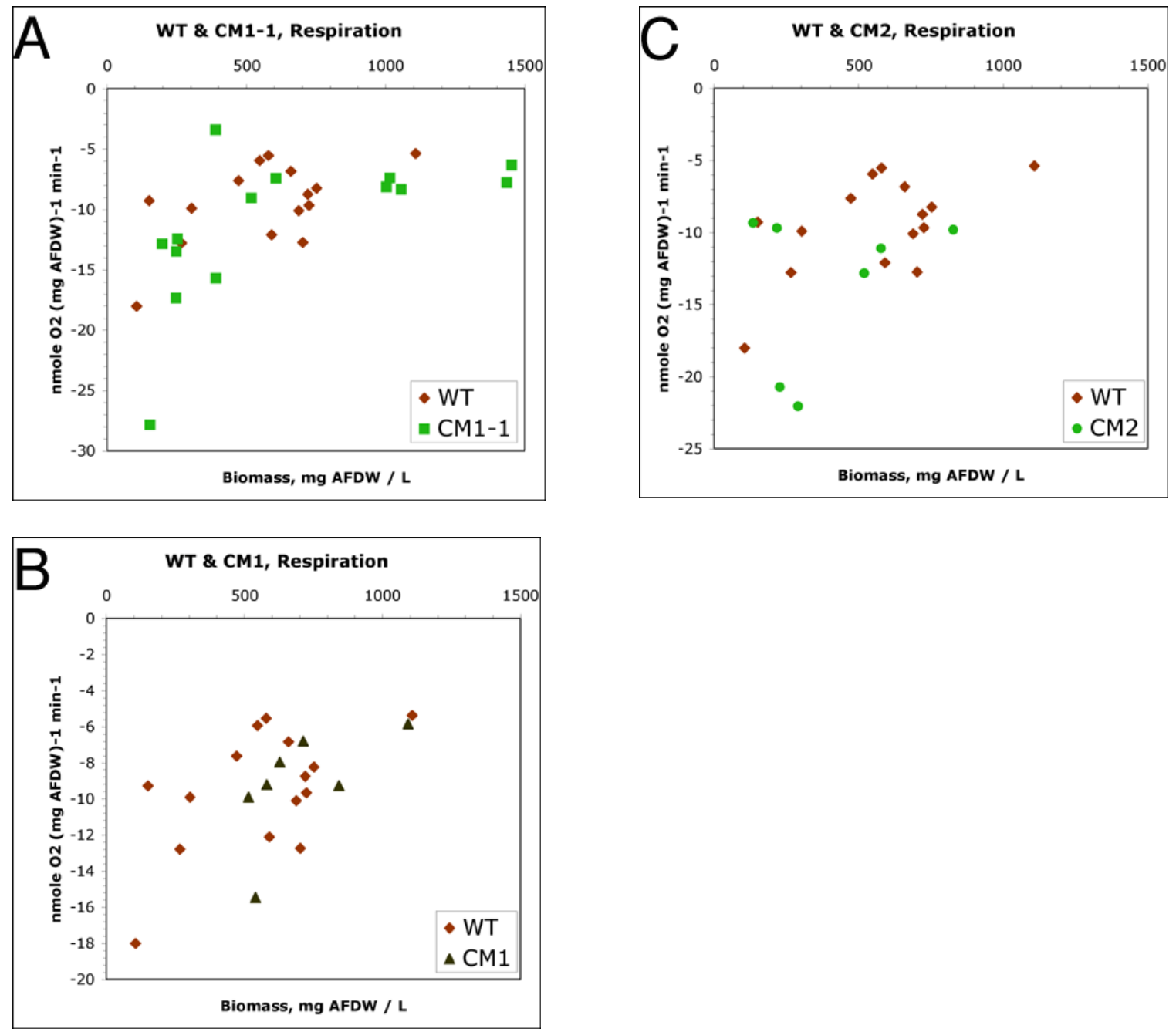

\section{Photosynthesis versus Irradiance Curves of WT and CM1-1}

To obtain more specific information about the photosynthetic efficiency of cells from batch cultures of WT and the mutant CM1-1, oxygen evolution in dependence of the light 
intensity was measured as described above, and the so called photosynthesis versus irradiance curves were plotted (Figure 6).

Figure 6: Plots of photosynthesis versus irradiance for Cyclotella spec. WT and mutant CM1-1: A) WT from the exponential growth phase, B) WT from the light limited growth phase, C) Comparison of WT exponential vs linear growth phase, D) CM1-1 exponential growth phase, E) Comparison of WT vs CM1-1 from the exponential growth phase.
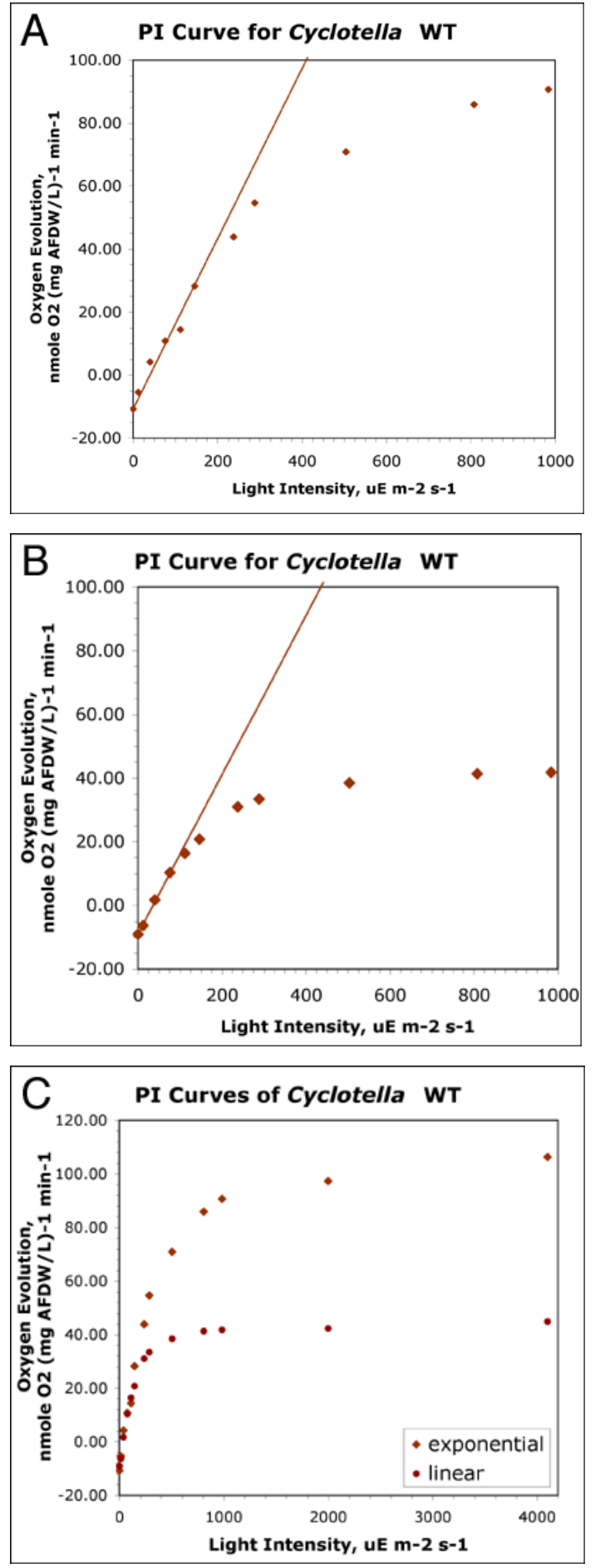
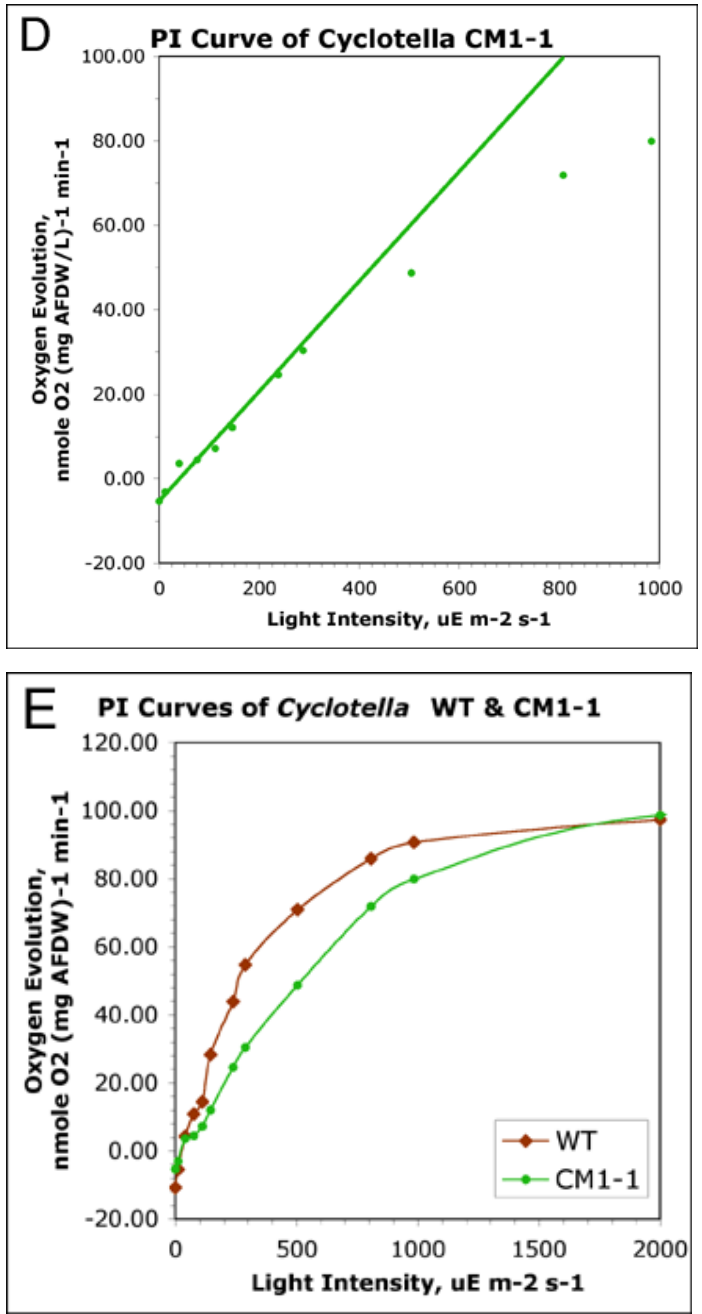
Figure 6A-C shows that PI curves of Cyclotella WT cells taken from either the exponential or the light limited growth phase of a batch culture have similar initial slopes. Cells taken from exponentially growing cultures of WT had a higher Pmax that those originating from the light-limited growth phase, indicating that the exponentially growing cells are acclimated to a higher light environment.

Exponentially growing cells of WT and the CM1-1 mutant had similar Pmax values, indicating that both have the same maximal photosynthetic capabilities and they should have similar growth rates. However, at the same time the initial slope of the PI curves of the CM1-1 mutant was lower than that of the WT (Figure 6E), suggesting that the mutant cells were affected for some unknown reason.

\section{Comparison of the growth for WT and Mutant CM1-1 batch cultures}

Comparable maximal photosynthetic rates between WT and mutant strains should indicate similar growth rates for all strains. To obtain direct information about the productivity of the mutant CM1-1 in comparison to the wild type simulated mass cultures of both strains were grown as described above. The increase in biomass was monitored by determining the Ash Free Dry Weight per liter of culture following incubation of new batch cultures. Several independent cultures were measured for WT and mutant CM1-1 and the raw data are shown in Figure 7. Cultures of the wild type had a very short lag and exponential growth phase and were light limited already after about 12 hours, which corresponded to a biomass concentration of approximately $200 \mathrm{mg}$ AFDW/L. In contrast, cultures of CM1-1 reached the light limited phase only after about 48 hours and had at that point a biomass concentration of about 300-400 $\mathrm{mg}$ AFDW/L. This result indicated that in the exponential growth phase the growth rate of CM1-1 was significantly lower than that of the wild type. Nevertheless, during the light limited growth phase wild type and CM1-1 had similar growth rates (see below). Figure 7 shows that the wild type reached the stationary phase at a biomass concentration of approximately $2,700 \mathrm{mg}$ AFDW/L whereas CM1-1 reached the stationary phase already at a much lower biomass concentration of about 1,800 mg AFDW/L. 
Figure 7: Shown are the growth curves for Cyclotella spec. WT and the CM1-1 mutant under simulated mass culture conditions. Data from six independent WT and CM1-1 cultures are displayed in the graph.

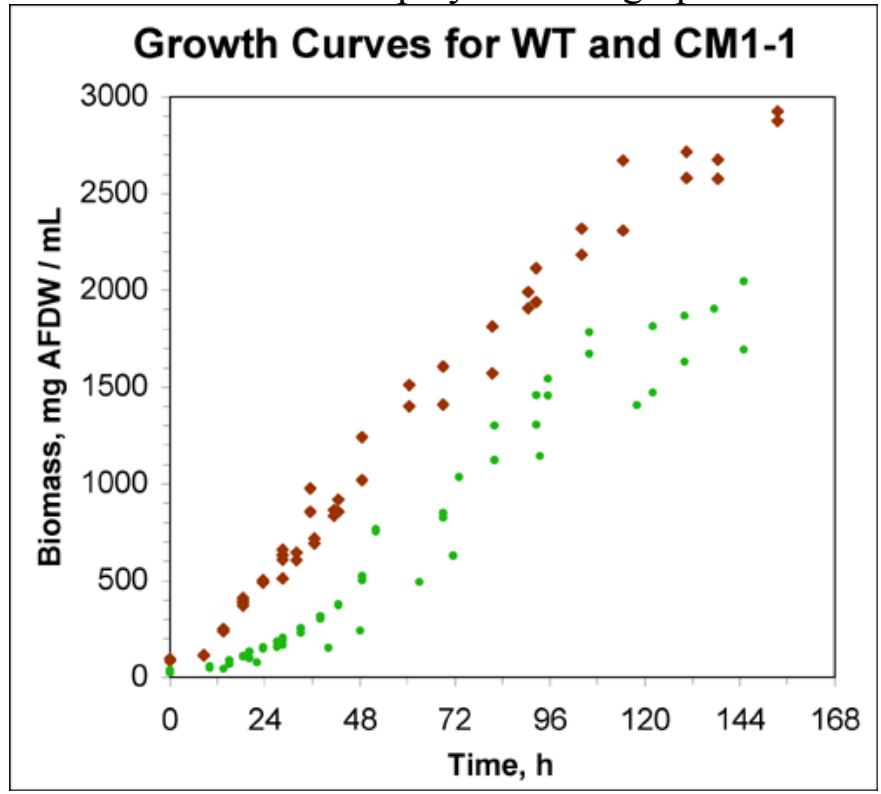

From the logarithmic plot of a growth curve the doubling time $(\mathrm{G})$ during exponential growth was calculated according to the following formula:

$$
\mathrm{G}=\frac{\text { Time }[\mathrm{min}]}{3.3 * \log [\mathrm{E} / \mathrm{B}]}
$$

With: $E=$ Biomass at the end of a time interval

$\mathrm{B}=$ Biomass at the beginning of a time interval

To determine the generation times for WT and mutant CM1-1 the growth data for all cultures were averaged (Figure 8A), and the logarithmic plot is shown in Figure 8B. For the given growth conditions, during the exponential growth phase the generation time $(\mathrm{G})$ for Cyclotella WT was 4.7 hours and for the mutant CM1-1 9.2 hours. For the light limited growth phase (500 - 1,000 mg AFDW/L) the generation time for Cyclotella WT was approximately 19.5 hours and for the CM1-1 mutant approximately 25 hours.

The growth rates during the light-limited phase determined from growth curves for WT and mutant CM1-1 are comparable. This result is in line with the comparable maximum biomass production rates calculated from Pmax values of cells of WT and CM1-1 mutant from the light-limited growth phase (Table 3). 
Figure 8: A) Shown are the averaged growth curves for Cyclotella spec. WT and CM1-1 from Figure 7. B) Logarithmic plot of the curves shown in panel A.
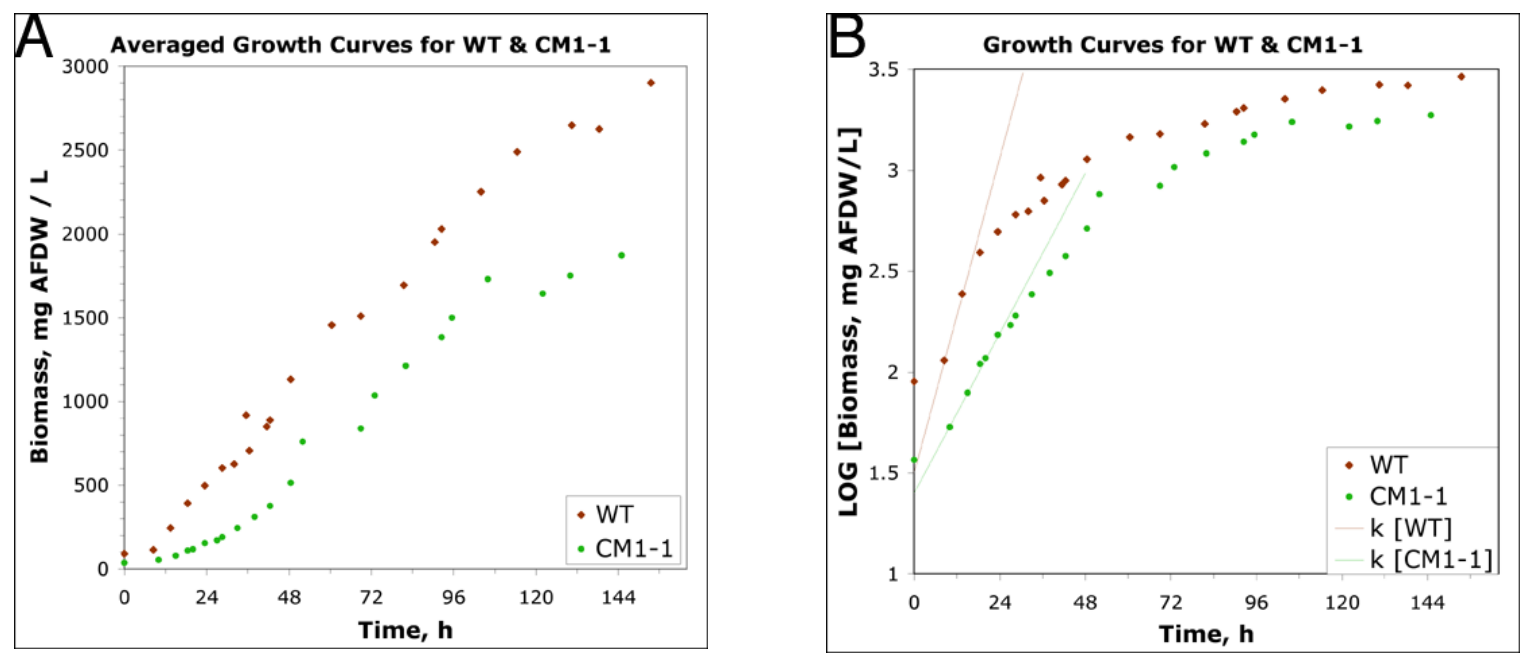

Figure 9: A) Shown are the growth curves for batch cultures of Cyclotella spec. WT and CM1-1 grown at $\sim 70 \mu$ mole photons $\mathrm{m}^{-2} \mathrm{~s}^{-1}$. Each curve is the average of four independent cultures. B) LOG plot of the growth curves shown in panel A.
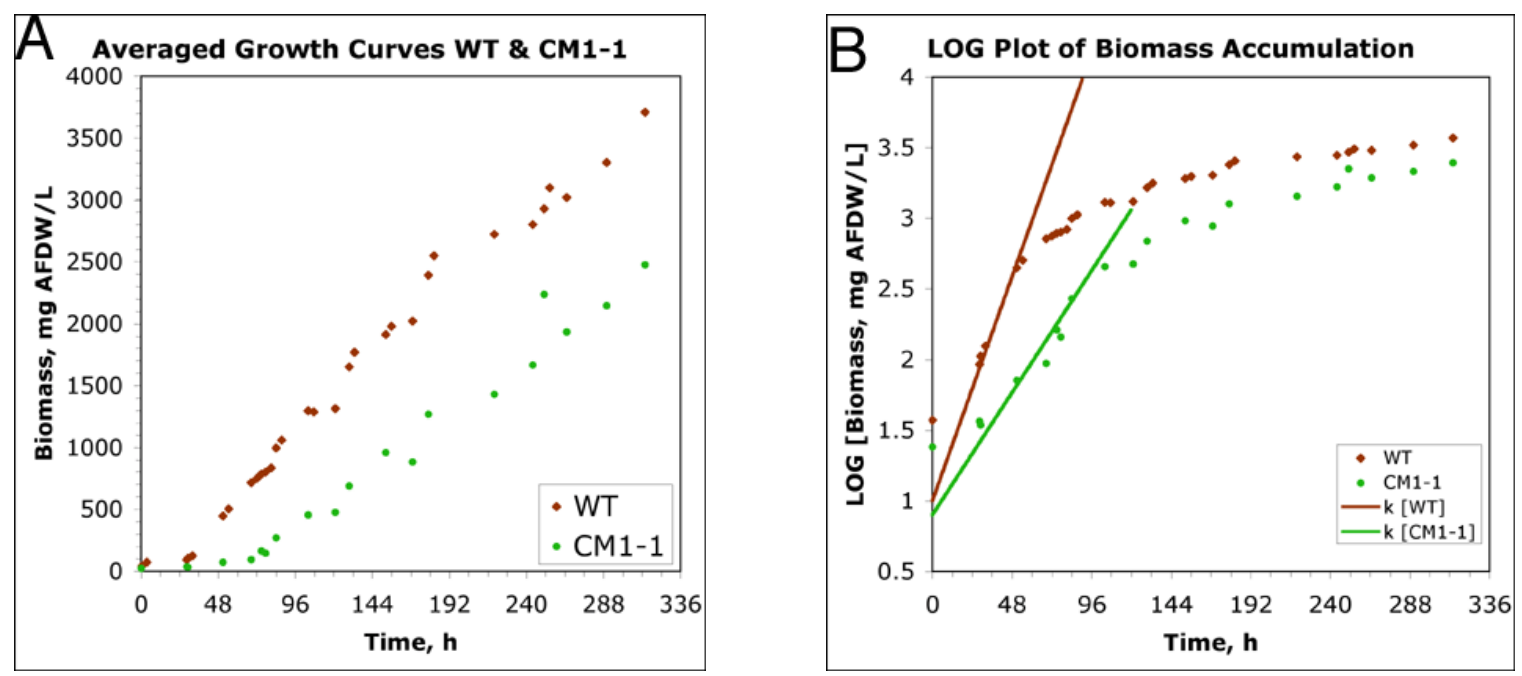

To further determine the dependency of biomass accumulation of wild type and CM11 on the light intensity during cultivation both strains were also grown under reduced light intensity. Growth of batch cultures was monitored as the increase in biomass (AFDW/L) over time at reduced growth light intensities $\left(\sim 70 \mu\right.$ mole photons $\left.\mathrm{m}^{-2} \mathrm{~s}^{-1}\right)$ and is shown in Figure 9A. Based on the LOG plot (Figure 9B) the generation time during the exponential growth phase was calculated for WT to be 9.9 hours and for CM1-1 to be 16.8 hours. Although the growth rate of CM1-1 was significantly lower than that of the 
WT during the exponential growth phase, during the light limited growth phase both WT and CM1-1 had similar growth rates.

Table 4 compares the generation times of WT and CM1-1 cells during the exponential growth phase. An increase in the growth light intensity from $70 \mu \mathrm{E} \mathrm{m} \mathrm{m}^{-2}$ to $170 \mu \mathrm{E} \mathrm{m}^{-2} \mathrm{~s}^{-1}$ resulted in reduction of the generation time by $\sim 50 \%$. The increase in growth light intensity reduced the generation time for mutant CM1-1 to a similar extent ( $60 \%)$. More studies with higher growth light intensities would be necessary to delineate, if mutant CM1-1 could perform as well or better than WT in the exponential growth phase.

Table 4: Comparison of the generation times of WT and CM1-1 cells during the exponential growth phase of a simulated mass culture.

\begin{tabular}{lcc}
\hline & $170 \mu \mathrm{E} \mathrm{m}^{-2} \mathrm{~s}^{-1}$ & $70 \mu \mathrm{E} \mathrm{m}^{-2} \mathrm{~s}^{-1}$ \\
\hline WT & $4.7 \mathrm{~h}$ & $9.2 \mathrm{~h}$ \\
CM1-1 & $9.9 \mathrm{~h}$ & $16.8 \mathrm{~h}$ \\
\hline
\end{tabular}

\section{Comparison of the Productivity for WT and Mutant CM1-1}

To obtain direct information about the productivity of the mutant CM1-1 in comparison to the wild type the growth data obtained in previous experiments were replotted on a basis of biomass accumulation per hour. As described above, the increase in biomass was monitored by determining the Ash Free Dry Weight per liter of culture following incubation of new batch cultures. Figure 10 compares the productivity data of three sets of WT and CM1-1 batch culture growth experiments. The exponential phase of WT cultures ends at about 24 hours. The exponential phase of CM1-1 cultures ends at about 48 hours. It is visible from Figures $10 \mathrm{~A}, 10 \mathrm{~B}$, and $10 \mathrm{C}$ that the productivity of CM1-1 cultures in the exponential phase is about 6 fold lower than that of WT cultures. Only when cultures of the CM1-1 mutant enter the light-limited growth phase at around 48 do they reach a level of productivity comparable to the WT.

Similar maximal photosynthetic rates of cells taken during growth of WT and CM1-1 cultures had indicated that both strains have similar photosynthetic capabilities during all growth phases. However, the data shown in Figure 10 demonstrated that the productivity of the CM1-1 mutant during the exponential growth phase was much lower than that of 
the WT and that their productivity matched only during the light-limited growth phase. This discrepancy between photosynthetic capability and actual productivity in the exponential growth phase suggested that cells of the mutant CM1-1 had some unknown lesion that affected growth. Considering that UV mutagenesis most probably introduces multiple mutations into cells, it is not unlikely that the CM1-1 mutant contains other mutations besides the pigment mutation that could affect its productivity.

Figure 10: Shown is a comparison of the productivity of batch cultures of the wildt type and the green pigment mutant CM1-1.
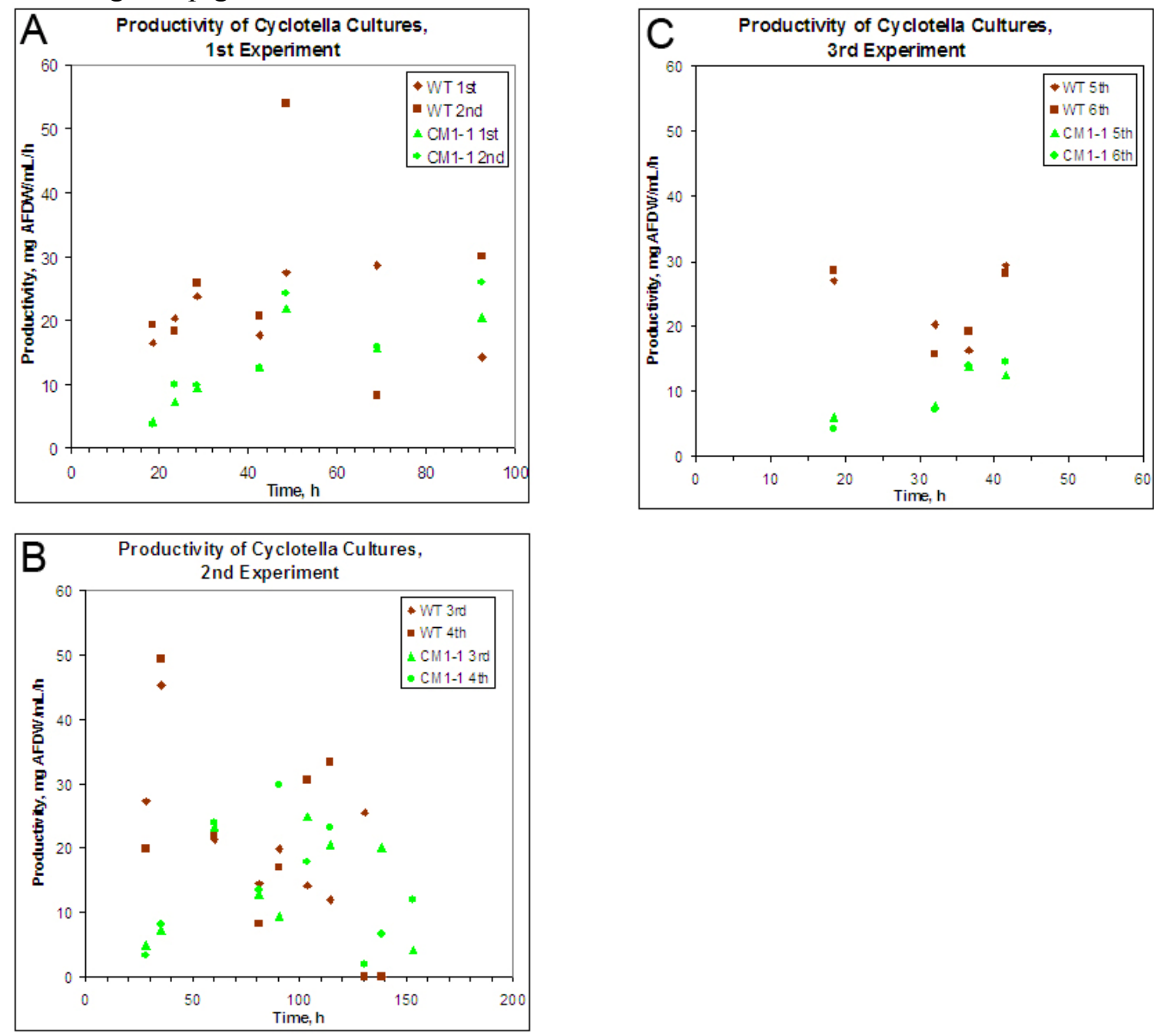

\section{Analysis of the Pigment Content of WT and the CM1-1 Mutant by Absorbance Spectra}

It is obvious from Figure 1 and from Figure 2 that the coloration of the mutants CM1, CM2, and CM1-1 was very different than that of the wild type, indicating altered pigment composition in the mutant cells. 
Figure 11: Absorbance spectra taken from intact cells of Cyclotella spec. WT and CM11 normalized to Chl $a$.

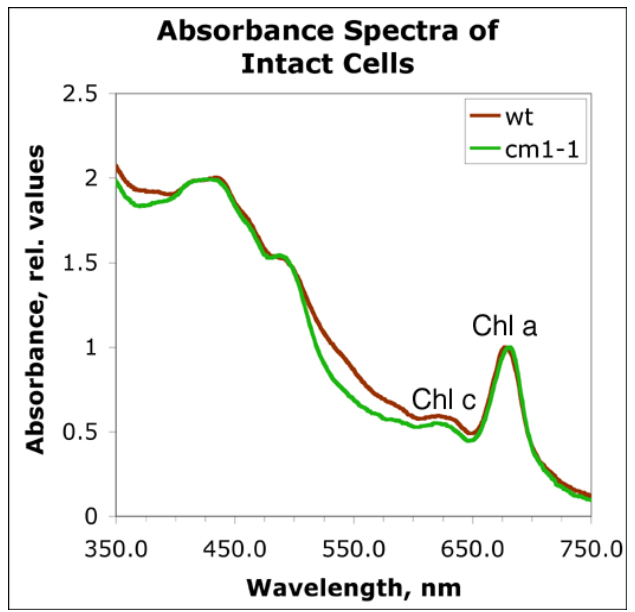

For intact cells of wild type and CM1-1 absorbance spectra were taken to obtain information about changes in chlorophyll and carotenoid contents. Figure 10 shows that on a per Chl $a$ basis, mutant CM1-1 contains less $\mathrm{Chl} c$ in comparison to WT. Figure 11 also shows that one shoulder at about $\lambda 550 \mathrm{~nm}$, associated with the carotenoid Fucoxanthin, was significantly less pronounced in the mutant CM1-1. This result strongly suggested that, in comparison to WT cells, CM1-1 had drastically reduced levels of Fucoxanthin.

Figure 12: Shown are liquid samples of Cyclotella spec. WT and CM1-1 cultures. The WT and the green sample of CM1-1 were taken from cultures that had approximately 1,000 mg AFDW/L. The brownish colored sample of CM1-1 was taken from a culture that had more than 2,000 $\mathrm{mg}$ AFDW/L.

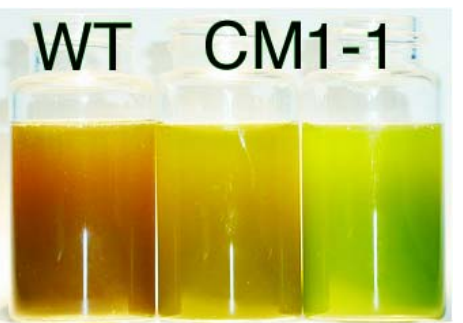

Although cultures of the mutant CM1-1 were green in the earlier growth phases, it was noticed that late in the light limited growth phase the coloration of cultures of CM1-1 changed from green to olive. Therefore, samples of the wild type and mutant CM1-1 were harvested in the early light limited growth phase of batch cultures containing about $1,000 \mathrm{mg}$ AFDW/L. At the same time green cells of the mutant CM1-1 were harvested 
from a culture containing approximately 2,000 mg AFDW/L. All samples are shown in Figure 12.

Figure 13: Absorbance spectra of $90 \%$ acetone extracts of cells of WT and CM1-1 normalized to $\mathrm{Chl} a$.

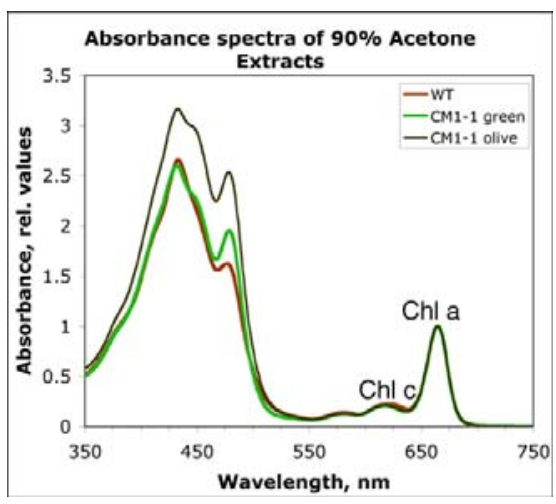

The change in coloration from green to olive-green late in the light limited growth phase of CM1-1 cultures strongly indicated that those cells accumulated carotenoids that might be associated with antenna proteins. To investigate this possibility $90 \%$ acetone extracts were prepared from cells of different growth phases (see above). Figure 13 shows that based on Chl $a$, green cells of mutant CM1-1 contained similar amounts of carotenoids as the WT. However, the difference in the carotenoid region of the spectra indicates that WT and CM1-1 have somewhat dissimilar compositions of carotenoids. Figure 13 also shows that based on Chl $a$, olive-green cells of CM1-1 had significantly higher peaks in the carotenoid region suggesting increased amounts of carotenoids. At the same time the overall shape of the curve of olive-green cells was similar to that of green CM1-1 cells, thus indicating comparable carotenoids composition.

In order to analyze the pigment composition of WT and CM1-1 more specifically, cells were harvested by centrifugation from samples taken from batch cultures in the light limited growth phase and were subjected to HPLC. However, the experiments were performed at Lehman College of CUNY, but no conclusive results could be obtained.

\section{Comparartive Protein Analysis and Immunoblotting}

Total cell proteins were isolated for Cyclotella wild type and the mutants CM1-1. Proteins were separated by SDS Gelelectrophoresis. However, the results were not 
conclusive and no antibodies against the light-harvesting FCP proteins of diatoms could be obtained.

\section{Summary}

Mutagenesis of Dunaliella bardawil did not result in generation of any potential chlorophyll antenna size mutants. Also mutagenesis of different strains of the unicellular green alga Tetraselmis spec. did not result in any potential antenna mutant. In contrast, through chemical and UV mutagenesis three pigment mutants of the diatom Cyclotella spec. (CM1, CM1-1, CM2) were isolated that are likely to have truncated chlorophyll antenna sizes of the photosystems. All three mutants have similar maximal photosynthetic rates as related to the wild type, when compared during growth in the light limited phase of a batch culture. However, it was determined that the CM1-1 mutant had a drastically reduced productivity during its exponential growth phase. Only in dense cultures during the light-limited growth phase did the CM1-1 mutant show comparable productivity to the WT.

In brief, the two specific aims to 1) generate microalgae mutants with truncated antenna size of the photosystems and to 2) compare the productivity of the wild type to that of the mutants were accomplished in this work.

In addition, the results of the preliminary mutant analysis have been published:

J.E.W. Polle, M. Aksoy, G. Stauffer, J.R. Benemann, and J.C. Weissman, "Genetic Improvements for Increasing Solar Energy Conversion Efficiency by Microalgae Cultures." Proceedings for the $13^{\text {th }}$ International Congress of Photosynthesis, Montreal, Canada 